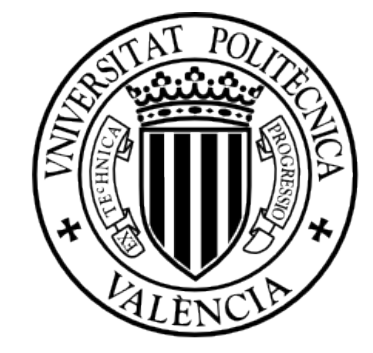

Doctoral Thesis

\title{
ANALYSIS OF PRIMARY ATOMIZATION IN SPRAYS USING Direct Numerical Simulation
}

\author{
Presented by: \\ Marco Crialesi Esposito \\ Supervised by: \\ Prof. Francisco Javier Salvador Rubio \\ in fulfillment of the requirements for the degree of \\ Doctor of Philosophy
}

Valencia, September 2019 



\section{Ph.D. Thesis}

\section{ANALYSIS OF PRIMARY ATOMIZATION IN SPRAYS USING Direct Numerical Simulation}

Written by: Mr. Marco Crialesi Esposito

Supervised by: Prof. Francisco Javier Salvador Rubio

Examination committee:

Chairman: $\quad$ Prof. Jose Maria Desantes Fernandez Secretary: Dr. Daniel Mira Martinez

Member: $\quad$ Prof. François-Xavier Demoulin

Reviewing board:

Prof. Michele Battistoni

Prof. François-Xavier Demoulin

Dr. Oriol Lehmkuhl Barba

Valencia, September 2019 



\section{Abstract}

The understanding of the physical phenomena occurring in the dense region (also known as near field) of atomizing sprays has been long seen as one of the biggest unknown when studying sprays applications. The industrial range of interest goes from nozzles in combustion and propulsion applications to medical sprays, agricultural and food process applications. This substantial lack of knowledge is responsible for some important simplification in modeling, that often result to be inaccurate or simply partial, leading to the evident need of large experimental characterization during the design phase. In fact, the spray breakup and primary atomization processes are indeed fundamental problems of physics, which complexity results from the combination of a multiphase flow in a highly turbulent regime that leads to chaotic scenarios.

The analysis of this problem is extremely problematic, due to a substantial lack of definitive theories about the physical phenomena involved, namely turbulence and atomization. Furthermore, the combination of the multiphase nature of the flow and its turbulent behavior makes substantially difficult to address the problem. Only within the last 10 years, experimental techniques have been capable of visualizing the dense region, but the experiments reliability, analysis and effectiveness in this region still requires vast improvements.

In this scenario, this thesis aims to contribute in the understanding of these physical process and to provide analysis tools for these complex flows. In order to do so, Direct Numerical Simulations have been used for addressing the problem at its smallest scale of motion, while reliably capturing all turbulence scales and breakup events. The multiphase nature of the flow is accounted for by using the Volume of Fluid method.

One of the goal of the thesis was to assess the influence of the inflow boundary conditions on the primary atomization and on the spray's turbulence behavior. In order to do so, two different boundary conditions were used. In a first place, a synthetic inflow boundary condition was used in order to produce a homogeneous turbulence inflow, simulating the nozzle behavior. One of the interesting features of this method was the possibility of tweaking the parameters within the algorithm. In particular, the integral length scale was varied in order to assess the influence of nozzle larger turbulent structures on the primary atomization.

The analysis on the synthetic boundary condition also allowed to optimally design simulations from which derive meaningful turbulence statistics. On this framework, further studies were carried over on the influence of turbulent structures properties, namely homogeneity and anisotropy, on both the flows spectra and droplets statistics. In order to achieve this goal, novel procedures 
for both computing the flow spectra and analyzing droplets were developed and are carefully addressed in the thesis.

The results of the analysis highlight the independence of droplets statistics from the inflow boundary condition, while, on the other hand, remarking how the total quantity of atomized mass is significantly affected by the turbulence features developed within the nozzle. This considerations are supported by the spectrum analysis performed, which also highlighted how multiphase turbulence shares the universal features described in Kolmogorov theories. 


\section{Resumen}

La comprensión de los fenómenos físicos que acontecen en la región densa (también conocida como campo cercano) durante la atomización de los sprays ha sido una de las mayores incógnitas a la hora de estudiar sus aplicaciones. En el sector industrial, el rango de interés abarca desde toberas en aplicaciones propulsivas a sprays en aplicaciones médicas, agrícolas o culinarias. Esta evidente falta de conocimiento obliga a realizar simplificaciones en la modelización, provocando resultados poco precisos y la necesidad de grandes caracterizaciones experimentales en la fase de diseño. De esta manera, los procesos de rotura del spray y atomización primaria se consideran problemas físicos fundamentales, cuya complejidad viene dada como resultado de un flujo multifásico en un régimen altamente turbulento, originando escenarios caóticos.

El análisis de este problema es extremadamente complejo debido a la ausencia sustancial de teorías validadas referentes a los fenómenos físicos involucrados como son la turbulencia y la atomización. Además, la combinación de la naturaleza multifásica del flujo y su comportamiento turbulento resultan en una gran dificultad para afrontar el problema. Durante los últimos 10 años, las técnicas experimentales han sido finalmente capaces de visualizar la región densa, pero la confianza, análisis y efectividad de dichos experimentos en esta región del spray todavía requiere de mejoras sustanciales.

En este contexto, esta tesis trata de contribuir al entendimiento de estos procesos físicos y de proporcionar herramientas de análisis para estos flujos tan complejos. Para ello, mediante Direct Numerical Simulations se ha afrontado el problema resolviendo las escalas de movimiento más pequeñas, y capturando todas las escalas de turbulencia y eventos de rotura.

Uno de los objetivos de la tesis ha sido evaluar la influencia de las condiciones de contorno del flujo entrante en la atomización primaria y en el comportamiento turbulento del spray. Para ello, se han empleado dos condiciones de contorno diferentes. En primer lugar se ha empleado una condición de contorno sintética para producir turbulencia homogenea a la entrada, simulando el comporamiento de la tobera. Una de las características más interesantes de este método es la posibilidad de retocar los parámetros dentro del algoritmo. En particular, la escala de longitud integral se ha variado para evaluar la influencia de las estructuras mas grandes de la tobera en la atomización primaria.

El análisis de la condición de contorno sintética también ha permitido el diseño óptimo de simulaciones de las cuales se han derivado estadísticas turbulentas significativas. En este escenario, se han llevado a cabo estudios 
más profundos sobre la influencia de propiedades de las estructuras turbulentas como la homogeneidad y la anisotropía tanto en el espectro de los flujos como en las estadísticas de las gotas. Para tal fin, se han desarrollado metodologías novedosas para computar el análisis espectral y la estadística de las gotas

Entre los resultados de este análisis destaca la independencia de la condición de contorno de entrada en las estadísticas de las gotas, mientras que por otra parte, recalca que las características turbulentas desarrolladas en el interior de la tobera afectan a la cantidad total de masa atomizada. Estas consideraciones se encuentran respaldadas por el análisis espectral realizado, mediante el cuál se concluye que la turbulencia multifásica comparte el comportamiento universal descrito por las teorías de Kolmogorov. 


\section{Resum}

La comprensió dels fenòmens físics que succeïxen en la regió densa (també coneguda com a camp pròxim) durant l'atomització dels sprays ha sigut una de les majors incògnites a l'hora d'estudiar les seues aplicacions. En el sector industrial, el rang d'interés comprén des de toveres en aplicacions propulsives a sprays en aplicacions mèdiques, agrícoles o culinàries. Esta evident falta de coneixement obliga a realitzar simplificacions en la modelització, provocant resultats poc precisos i la necessitat de grans caracteritzacions experimentals en la fase de disseny. D'esta manera, els processos de ruptura del spray i atomització primària es consideren problemes físics fonamentals, la complexitat dels quals ve donada com resultat d'un flux multifàsic en un règim altament turbulent, originant escenaris caòtics.

L'anàlisi d'este problema és extremadament complex a causa de l'absència substancial de teories validades dels fenòmens físics involucrats com són la turbulència i l'atomització. A més, la combinació de la naturalesa multifàsica del flux i el seu comportament turbulent resulten en una gran dificultat per a afrontar el problema. Durant els últims 10 anys les tècniques experimentals han sigut finalment capaces de visualitzar la regió densa, però la confianÃßa, anàlisi i efectivitat dels experiments en esta regió del spray encara requerix de millores substancials.

En este context, esta tesi tracta de contribuir en l'enteniment d'estos processos físics i de proporcionar ferramentes d'anàlisi per a estos fluxos tan complexos. Per a això, per mitjà de Direct Numerical Simulations s'ha afrontat el problema resolent les escales de moviment més menudes, al mateix temps que es capturen totes les escales de turbulència i esdeveniments de ruptura.

Un dels objectius de la tesi ha sigut avaluar la influència que les condicions de contorn del flux entrant tenen en l'atomització primària i en el comportament turbulent del spray. Per a això, s'han empleat dos condicions de contorn diferents. En primer lloc s'ha empleat una condició de contorn sintètica per a produir turbulència homogènia a l'entrada, simulant el comportament de la tovera. Una de les característiques més interessants d'este mètod és la possibilitat de retocar els paràmetres dins de l'algoritme. En particular, l'escala de longitud integral s'ha variat per a avaluar la influència de les estructures mes grans de la tovera en l'atomització primària.

L'anàlisi de la condició de contorn sintètica també ha permés el disseny òptim de simulacions de les quals s'han derivat estadístiques turbulentes significatives. En este escenari, s'han dut a terme estudis més profunds sobre la influència de propietats de les estructures turbulentes com l'homogeneïtat i l'anisotropia tant en l'espectre dels fluxos com en les estadístiques de les 
gotes. Per a tal fi, s'han desenrotllat metodologies noves per a computar l'anàlisi espectral i l'estadística de les gotes.

Entre els resultats d'esta anàlisi destaca la independència de la condició de contorn d'entrada en les estadístiques de les gotes, mentres que d'altra banda, es recalca que les característiques turbulentes desenrotllades en l'interior de la tovera afecten a la quantitat total de massa atomitzada. Estes consideracions es troben recolzades per l'anàlisi espectral realitzat, per mitjà del qual es conclou que la turbulència multifásica compartix el comportament universal descrit per les teories de Kolmogorov. 
To my family and my friends. 

There is a number of people that I want to thank for helping me through all the steps I needed to take in order to be able to finish this document. I would like to thank all the people at CMT-Motores Termicos at Universitat Politecnica de Valencia, for assisting me and helping me during these years. From people in administration, who always helped me out with any bureaucratic procedure, to all Professors and students, who were always ready to help me out in any moment of difficulty, both with technical and moral support. My deepest gratitude goes to Prof. Pedro Marti, Prof. Marcos Carreres, Prof. Jaime Gimeno, Prof. Antonio Torregrosa, Dr. Alberto Viera, Prof. Antonio Gil and Dr. Jose-Manuel Pastor. I would also like to thanks Jose Enrique Del Rey, Pau Raga and Juan Antonio Yustas for their technical (and, often, moral) support. Finally, I would like to acknowledge Lucas Gonzalez and Mario Belmar, my office mates, for their help and patience in dealing with me through the ups and downs a PhD thesis inevitably puts you through.

A special thanks to Prof. Raul Payri, for putting me always in position to work and perform at my best, providing all sorts of resources needed for successfully complete my PhD. Finally, a huge thanks goes to my supervisor, Prof. Javier Salvador, for his mentoring, his constant presence, advice and support. Thanks for being an example of hard-working and for helping me finishing my thesis, always redirecting the research towards more interesting analysis when needed.

I would really like to thank Prof. Stephane Zaleski, Dr. Stephane Popinet, Dr. Daniel Fuster and Dr. Wojtek Aniszewski for the help they provided me in growing as a scientist and for involving me in great scientific discussions with many peoples at Institut Jean Le Rond d'Alembert.

A huge thank you to all my friends, with a special mention to my life-time friends from Parma. While far geographically they have always find the way to be close to me in my daily life. Grazie ragazzi...

Finally, my utmost gratitude for everything (not only these last 4 years...) goes to my family, my wonderful parents and my incredible sister! Non ci sono parole per dirvi quanto vi sia grato e quanto il vostro supporto incodizionionato a ogni mia decisione sia stato fondamentale nel raggiungere questo traguardo. Un enorme grazie per il vostro aiuto, in qualsiasi aspetto della mia vita, dal più banale al più profondo. 



\section{Contents}

Contents

List of Figures $\quad$ v

List of Tables $\quad$ x

Nomenclature $\quad$ xi

1 Introduction $\quad \mathbf{1}$

1.1 General context ..................... 1

1.2 Latest developments . . . . . . . . . . . . . . . . 7

1.2.1 Experimental techniques in the dense region. . . . . . 8

1.2.2 DNS simulation of sprays in literature . . . . . . . . 18

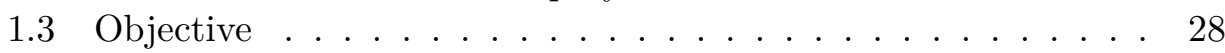

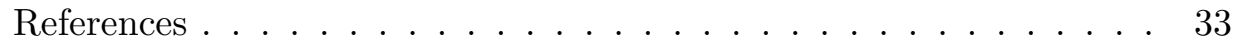

2 Overview of numerical methods in multiphase flows. 43

2.1 Numerical framework requirements . . . . . . . . . . . . 43

2.2 Basic equations for multiphase flows . . . . . . . . . . . . 46

2.3 Solution of the Navier-Stokes equation . . . . . . . . . . . . . 47

2.3.1 The Chorin projection method . . . . . . . . . . . 48

2.3.2 Spatial integration ................. 49

2.3.3 Viscous term ................. 51

2.3.4 Methods for solving the pressure equation . . . . . . . 51

2.4 Interface treatment . . . . . . . . . . . . . . . . . . . 53

2.4.1 Defining a surface and its advection . . . . . . . . 53

2.4.2 Treating the surface tension terms . . . . . . . . 58 
2.4.3 Height Function method . . . . . . . . . . . . . . 59

2.5 Overview of the methods used within ParisSimulator . . . . . . 61

References . . . . . . . . . . . . . . . . . 62

3 Treatment of the inflow boundary conditions $\quad 67$

3.1 Synthetic inflow boundary conditions . . . . . . . . . . . 67

3.1.1 Review of available methods . . . . . . . . . . . . 67

3.1.2 Implementation of the synthetic turbulence inlet boundary conditions . . . . . . . . . . . . . . . 69

3.1.3 Velocity Inlet Profile and Turbulence Distribution . . . 71

3.2 Mapped boundary conditions . . . . . . . . . . . . 73

3.2.1 Methodology and analysis for the LES simulation . . . . 74

3.2.2 Turbulent intensity and lengthscale extracion for the SBC case . . . . . . . . . . . . . . 78

3.3 Resume and open questions . . . . . . . . . . . . . . . . 81

References . . . . . . . . . . . . . . . . . . 82

4 Methodologies for simulations analysis of turbulence and droplets.

4.1 An overview of turbulence theory. . . . . . . . . . . . . 85

4.1.1 Background on turbulence . . . . . . . . . . . . 87

4.2 An overview of droplet analysis in DNS . . . . . . . . . . . 95

4.3 Methodology for turbulence analysis in DNS . . . . . . . . . 97

4.4 Methodology for droplet analysis in DNS . . . . . . . . . . . 104

References . . . . . . . . . . . . . . . . . . 108

5 Influence of integral length scale on primary atomization 113

5.1 Introduction and case description . . . . . . . . . . . 113

5.2 Effects of the Synthetic Boundary Conditions . . . . . . . . 116

5.3 Observation on the Spray Morphology . . . . . . . . . . . . 121

5.3.1 Consideration on the Axial Spray Penetration . . . . . . 126

5.4 Observations on the atomization process . . . . . . . . 126

5.5 Analysis of droplets generation . . . . . . . . . . . 130

5.6 Observation on Gaussian behavior of the spray . . . . . . . . 133

5.7 Main conclusions . . . . . . . . . . . . . . . . . . 135

References . . . . . . . . . . . . . . . . 137

6 Analogies between multiphase and single-phase turbulence 141

6.1 Statistics convergence and analysis. . . . . . . . . . . . . 142

6.1.1 Reasons for the post-process design . . . . . . . . . . 142

6.1 .2 Statistics convergence . . . . . . . . . . . . . . 144 
6.2 The pseudo-fluid approach. . . . . . . . . . . . . 146

6.3 Autocorrelation and integral lengthscale. . . . . . . . . . 150

6.4 Kolmogorov spectra scaling. . . . . . . . . . . . . . . . 152

6.5 Conclusions. . . . . . . . . . . . . . . . . . 155

References . . . . . . . . . . . . . . . . 155

7 Effects of isotropic and anisotropic turbulent structures on primary atomization $\quad 159$

7.1 Mean fields analysis using one-point statistics . . . . . . . . 160

7.2 Determination of the Kolmogorov scale . . . . . . . . . . . . . . 164

7.3 Spectral analysis of turbulence . . . . . . . . . . . 166

7.4 Droplet size and properties distribution . . . . . . . . . 170

7.5 Droplet radial position . . . . . . . . . . . . . . . 175

7.6 Spray axial development . . . . . . . . . . . . . 178

7.7 Generation of droplets over time . . . . . . . . . . . 180

7.8 Radial Distribution Function analysis. . . . . . . . . . . . . 183

7.9 Conclusions . . . . . . . . . . . . . . . . . . 185

References . . . . . . . . . . . . . . . . . 186

8 Conclusion and future works $\quad 189$

8.1 Conclusion over physics analysis . . . . . . . . . . . 190

8.1.1 Considerations over the inflow boundary conditions . . . 190

8.1.2 Considerations over the analysis of droplets properties . 191

8.1.3 Consideration on multiphase turbulence . . . . . . . . 193

8.1.4 How far is DNS from reality? . . . . . . . . . . . . . 194

8.2 Development of a post processing toolbox . . . . . . . . 195

8.2.1 Boundary conditions in ParisSimulator . . . . . . . . 196

8.2.2 Datasets storing and handling . . . . . . . . . . . 196

8.2.3 Parallel post-process . . . . . . . . . . . . . . . 197

8.2.4 ParisSimulator performance improvement using GPU . 197

8.3 Future works . . . . . . . . . . . . . . . . . . 198

8.3.1 Using X-Rays and DNS for studying sprays . . . . . . 198

8.3.2 Analysis of Reynolds, Weber and density ratio influence over the atomization process _ . . . . . . . . 198

8.3.3 Analysis of aeroengines airblast atomizers using DNS . 199

8.3.4 Analysis of atomization causality in relation to turbulence scale . . . . . . . . . . . . . . . . . . 199

References . . . . . . . . . . . . . . . . . . . 199

$\begin{array}{ll}\text { A GPU Poisson-Solver } & 203\end{array}$ 
Global Bibliography 205 


\section{List of Figures}

1.1 Sketch of different atomization regimes. . . . . . . . . . . . 3

1.2 Sketch of a atomized spray main regions. . . . . . . . . . . 4

1.3 Sketch from Linne of light scatter through opaque mediums. . . . 8

1.4 Sketch from Linne resuming the theory of ballistic imaging both from a geometrical standpoint (left) and a time-wise stand-point

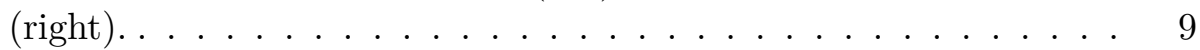

1.5 Data from Linne as an example of ballistic imaging results. . . . . 10

1.6 Sketch fo SLIPI technique setup from Linne . . . . . . . . . . . . . 10

1.7 Sketch fo SLIPI technique setup from Berrocal . . . . . . . . . . . 11

1.8 Realization of optical connectivity, schematic (left) and actual implementation in a real automotive multi-orifice nozzle. . . . . . . . 12

1.9 Example of results from optical connectivity experiments, from Charalampous. A shadowgraphy result is showed on the left, while its corresponding optical connectivity image is showed on the right

1.10 Render of the experimental setup for Near-Field microscopy, from

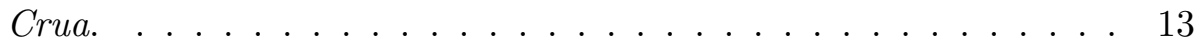

1.11 Initial transient of a diesel spray, from Crua. . . . . . . . . . . . 14

1.12 Sketch of the experimental layout used by Wang to perform x-ray PCI. . . . . . . . . . . . . . . . . . . . 14

1.13 Results that can be produced by x-rays PCI. . . . . . . . . . . 16

1.14 Sketch of the experimental layout used by Kastengren to perform x-ray radiography. . . . . . . . . . . . . . . 17

1.15 Results of experiments performed using x-ray radiography . . . . . 18

1.16 Results from Battistoni et al. of experiments performed using USAXS 19

1.17 Type of DNS simulations of sprays. Figure 1.17(c) is a render from Vaudor. . . . . . . . . . . . . . 20 
1.18 Results from Menard.Selection of ad-hoc region of different mass concentration (here described by means of the curvature distribution). . . . . . . . . . . . . . . . . . .

1.19 Results from Shinjo. The top figure shows how the turbulent structures propagates and decay upstream the spray core, while the lower one shows the plain render of the liquid core. . . . . . . . . . 24

1.20 Type of DNS simulations of sprays. Figure 1.17(c) is a render from Vaudor. . . . . . . . . . . . . . . . . . .

1.21 Results from Herrmann. Different data sets have different symbols for different RLSG grid-size, with a standard grid of size $\Delta x=$ $1.56 \mu \mathrm{m}$. The RGLS values are: triangles $1.56 \mu \mathrm{m}$, circles $0.78 \mu \mathrm{m}$ and squares $0.39 \mu \mathrm{m}$. . . . . . . . . . . . . . . . .

2.1 ParisSimulator code scalability on Marconi and MareNostrum4 supercomputers. . . . . . . . . . . . . . . . . .

2.2 Different method for surface reconstruction in VOF (from Tryggvason). a) is the original interface, b) is the SLIC reconstruction, $\mathbf{c}$ ) is the reconstruction method proposed by Hirt and $\mathbf{d}$ ) is the PLIC reconstruction. ..................... 56

2.3 Simple $3 \times 7$ stencil for the calculation of $h$, from Afkhami . . . . . 60

3.1 Axial velocity profile in a turbulent pipe, from Eggels . . . . . . . 71

3.2 Axial velocity fluctuation profile in a turbulent pipe, from Eggels . 72

3.3 Wall law, plotted using the dimensionless mean velocity. . . . . . . 75

3.4 Wall-law for the velocity root mean squared velocity component. . 76

3.5 Turbulent structures captured by the Q criterion as described in Jeong. . . . . . . . . . . . . . . . . . . . . . . 77

3.6 Inlet boundary condition for the MBC case. On the left the interpolated result to be fed into ParisSimulator and the mesh, on the right the LES corresponding result and the mesh points in red. All

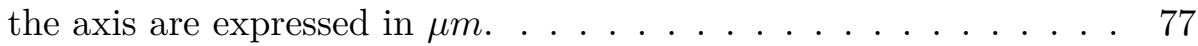

3.7 Autocorrelation for the LES simulation of pipe flow. . . . . . . . . 80

3.8 Turbulent integral lengthscale as a function of the radial position in the pipe flow. . . . . . . . . . . . . . . . . . . 80

4.1 Schematic sketch of an energy cascade with the relevant lengthscales. 89

4.2 Time-average axial velocity field over the analysis plane at $x / D=$ 25 for the MBC case (that will be discussed in more details later on). The lines represents the iso-radius curves over which the flow presents a statistical stationary behavior. . . . . . . . . . . 100

4.3 Results of the droplet detection procedure at $t=T_{t} / 4$ with $C_{t h}=0.4106$ 
4.4 Parameterization of the $C_{t h}$ parameter during the statistically stationary part of the simulation (from $T_{t}$ until $T_{t}+T_{s s}$ ) $\ldots 107$

5.1 Schematic representation of the spray, presenting the nomenclature used in the analysis. . . . . . . . . . . . . . . 116

5.2 Spray behavior in the transient spray under the synthetic boundary condition effects. Left to right case 0,1 and 2 . . . . . . . . 117

5.3 Simplified scheme of the distribution of aerodynamic drag forces and vorticity field around the spray tip and core . . . . . . . 118

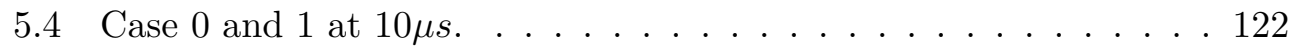

5.5 Turbulent structures detected through the Q-criterion for cases 0 and 1 at $10 \mu \mathrm{s} . \ldots \ldots \ldots \ldots \ldots \ldots \ldots \ldots$

5.6 External aspect of the injected spray at $t=20 \mu \mathrm{s}$. From top to bottom, case 0,1 and $2 \ldots \ldots \ldots \ldots \ldots$

5.7 Axial time-averaged mass concentration . . . . . . . . . . . . 125

5.8 Detailed view of the vortex lines over the spray core at $t=65 \mu \mathrm{s}$. In red, the vortex lines belonging to the same rim are highlighted.

5.9 Time sequence of spray formation. The three planes show the liquid evolution at $20 \mu \mathrm{s}, 20.5 \mu \mathrm{s}$ and $21 \mu \mathrm{s}$ (respectively from top to bottom) for case 1. . . . . . . . . . . . . . . . 129

5.10 Droplet statistics for the 3 cases. . . . . . . . . . . . . . . 131

5.11 Self-similar profile obtained for case 1 at $x / D=22 \ldots \ldots \ldots 134$

6.1 Render of the simulation described in Tables 6.1 and 6.2 and analyzed in this chapter. The VOF is contoured and colored by the local velocity. The shadowed green plane shows the position of the domain over which statistics have been analyzed. . . . . . . . . . 143

6.2 Convergence of the probability function statistics for axial velocity and its axial derivative. . . . . . . . . . . . . . 145

6.3 Probability function as a function of radial position. . . . . . . . . 147

6.4 Time-average volume fraction as a function of the radial position. . 149

6.5 Average energy dissipation rate terms, as presented in Equation (6.1).150

6.6 Kolmogorov scale as a function of the radial position. . . . . . . . 151

6.7 Autocorrelation function $f_{11}$ for the $\mathrm{MBC}$ case as a function of the dimensionless azimuthal coordinate $\xi$. In the legend each curve is described by its radial position $\left(r / r_{1 / 2}\right)$, its Reynolds-Taylor $R e_{\lambda}$ number and its Kolmogorov scale $\eta$. . . . . . . . . . . . . 152

6.8 Integral lengthscale $L_{11}$ as a function of the dimensionless radial position $\left(r / r_{1 / 2}\right) \ldots \ldots \ldots \ldots \ldots \ldots \ldots \ldots \ldots$ 
6.9 One dimensional energy spectra showed using the Kolmogorov scaling. Each radius is made dimensionless using its respective Kolmogorov scale in Figure 6.6 and the respective $\epsilon$ (Figure 6.5) and $\nu$. The data are from Uberoi 1970, Saddoughi 1994, Harris 1977, Comte-Bellot 1971. . . . . . . . . . . . . . . . . 15

7.1 Time averaged fields. . . . . . . . . . . . . . . . . . 162

7.2 Time averaged fields. . . . . . . . . . . . . . . . . 163

7.3 Energy dissipation rate. . . . . . . . . . . . . . . . 165

7.4 Time averaged turbulent fields. . . . . . . . . . . . . . . 167

7.5 Axial fluctuating velocity autocorrelation. Each plot represents a radial position, expressed both in metrical values (in the title) and dimensionless value (in the legend). Legend also report the local value of $R e_{\lambda}$ and $\eta$ for reference with previous figures. . . . . . . . 168

7.6 Axial fluctuating velocity autocorrelation. Each plot represents a radial position, expressed both in metrical values (in the title) and dimensionless value (in the legend). Legend also report the local value of $R e_{\lambda}$ and $\eta$ for reference with previous figures. . . . . . . . 171

7.7 Probability of droplet droplet size distribution. . . . . . . . . . . . 172

7.8 Probability of droplet kinetic energy distribution. The kinetic energy is made dimensionless by dividing by the injection kinetic energy $E_{i n j}=1 / 2\langle U\rangle^{2}$. . . . . . . . . . . . . . . . . . . 173

7.9 Joint PDF of droplet diameter $d_{v}$ and dimensionless kinetic energy $E_{k}^{*}$. . . . . . . . . . . . . . . . . 174

7.10 Time averaged fields. . . . . . . . . . . . . . . . . 176

7.11 Joint PDF for the droplet radial position $r$ (normalized by its axial position $x$ ) and axial position $x$ (normalized by its axial total length $\left.l_{x}\right)$. . . . . . . . . . . . . . . . . . . .

7.12 From top to bottom . (top) Probability $\mathcal{P}(x)$ of droplet presence as a function of axial position . (middle and bottom) Simulations snapshots of the volume fraction as a function of the radial position as well as the axial distance, both normalized by the nozzle diameter $D_{n}$. The x-axis of the figures are synchronized. . . . . . . 178

7.13 Probability $\mathcal{P}\left(d_{v}\right)$ for droplet size. The lines go from gray to black for $x \rightarrow l_{x}$. The axial distance goes from $x / D_{n}=2.5$ to $x / D_{n}=25$ according to the grayscale . . . . . . . . . . . . . . . . 179

7.14 Total droplet mass divided by droplet size range, displayed as a function of time. The 2 plots represents the same study at different ranges and have been divided for clarity. . . . . . . . . . . . . . . 180

7.15 Averaged droplets generation rate as a function of the droplet diameter. 
7.16 Total mass of atomized droplets over the total mass injected as a function of time. . . . . . . . . . . . . . . . . 183

7.17 RDF for droplets ranges (reported in legend). On the left, the results of the $\mathrm{MBC}$ case are showed while the results of the SBC case are showed on the right. . . . . . . . . . . . . . 184 


\section{List of Tables}

$5.1 \quad$ Simulation physical parameters . . . . . . . . . . . . . . . 114

5.2 Inlet turbulence model parameters for all the cases simulated . . . 115

5.3 Ligament parameters of the ligaments highlighted in Figure 5.9 . . 130

6.1 Physical simulation properties for the case studied. . . . . . . . . . 142

6.2 Simulation setups. . . . . . . . . . . . . . . . . . . . . 142

7.1 Simulation lumped quantities ratio summary. . . . . . . . . . . . 172 


\section{Nomenclature}

\section{Acronyms}

BC Boundary Condition.

BiCGSTAB Bi Conjugate Gradient Stabilized.

CFD Computational Fluid Dynamics.

CFL Courant-Friedrichs-Lewy number.

CIAM Calcul d'Interface Affine par Morceaux.

CSF Continuous Surface Force.

CSS Continuous Surface Stress.

DBI Diffused Backlight Illumination.

DNS Direct Numerical Simulation

FT Front Tracking.

GFM Ghost Fluid Method.

GPU Graphic Processing Unit.

HF Height Function.

HPC High Performance Computing.

LES Large Eddy Simulation.

LPP Lagrangian Point Particle.

LS Level-set.

MBC Mapped Boundary Condition.

OD Optical Density.

PARIS PArallel Robust Interface Simulator.

PCI Phase Contrast Imaging. 
PDF Probability Density Function.

PIV Particle Image Velocimetry.

PLIC Piecewise Linear Interface Calculation.

RANS Raynolds-Averaged Navier-Stokes.

RDF Radial distribution function.

SBC Synthetic Boundary Condition.

SBC Synthetic boundary condition.

SGS Sub-Grid Scales.

SLIC Simple Line Interface Calculation.

SLIPI Structured laser illumination planar imaging.

SMD Sauter Mean Diameter.

TKE Turbulent Kinetic Energy.

VOF Volume of Fluid.

WALE Wall-Adaptive Local Eddy-viscosity.

\section{Greek symbols}

$\alpha_{F} \quad$ Level-Set surface thickness.

$\beta \quad$ Mass diffusivity.

$\delta \quad$ Dirac Function.

$\epsilon \quad$ Mass diffusivity.

$\eta \quad$ Kolmogorov lengthscale.

$\eta_{B} \quad$ Batchelor lengthscale.

$\eta_{H} \quad$ Hinze lengthscale.

$\iota \quad$ Iteration step.

$\kappa \quad$ Surface curvature.

$\lambda_{l} \quad$ Ligament wavelength.

$\mu \quad$ Dynamic Viscosity.

$\nu \quad$ Kinematic Viscosity.

$\rho \quad$ Density.

$\sigma \quad$ Surface Tension.

$\Xi \quad$ Integral radial mass.

$\xi \quad$ Dimensional radial distance function.

\section{Latin symbols}

$\mathcal{A} \quad$ Advection term. 
$\mathcal{D} \quad$ Diffusion term.

$\mathcal{F} \quad$ Forces source term.

$\mathcal{L} \quad$ Characteristic flow lengthscale.

$b \quad$ Filter coefficient function.

$C \quad$ VOF color function.

$C_{h} \quad$ Hinze costant.

$D_{l} \quad$ Ligament diameter.

$D_{n} \quad$ Nozzle/pipe diameter.

$F \quad$ Level-Set function.

$f \quad$ normalized correlation function.

I Turbulent intensity.

$L \quad$ Turbulent integral length scale.

$L_{i c} \quad$ intact core length

$L_{n p} \quad$ External non-perturbed length.

$p \quad$ pressure.

$R \quad$ Correlation function.

$r \quad$ Random field.

$r_{d} \quad$ Distance vector.

$r_{1 / 2} \quad$ radial position where $\langle U\rangle=U_{c} / 2$.

$U \quad$ Velocity.

$u \quad$ Fluctuating velocity.

$U_{c} \quad$ Averaged centerline axialvelocity.

\section{Subscripts}

$\begin{array}{ll}g & \text { Gas phase. } \\ l & \text { Liquid phase. }\end{array}$





\section{Chapter 1}

\section{Introduction}

\subsection{General context}

Multiphase flows are ubiquitous in nature and extremely common in industrial contexts. As one of the most diffused branch of these flows, atomizing sprays are found in automotive sector, aeronautical applications and energy generation [1]. In these sectors, sprays are often used to breakup liquid fuels into fuel droplets that eventually will be burned in order to generate heat. Being environment one of the most important concerns while designing engineering applications, pollutants need to be kept at a minimum value, hence droplets diameter need to be predictable and controllable in injection devices.

The interest of sprays on the other hand goes far beyond the combustion realm. For example, in medical drug-delivery devices [2], such as inhaler, sprays are fundamental in order to have a correct delivery and dosage of the drug on inside patient. Spray particles size, whether liquid or solid, is crucial in determining the drug penetration during administration (for example in lungs) and the chances of its absorption. Moreover, in pharmaceutical applications, spray-drying systems are well known to be one of the most affordable and effective way to produce the powder composite needed in order to manufacture drugs [3].

Examples of sprays manifestation in nature are also extremely interesting. Diseases spreads are strictly related to sprays, in particular by the droplets dynamic of sneezes which are responsible for the transmission of most pathogens [4]. In this context, the spray penetration is directly related to the contam- 
ination range and its turbulence field may promote buoyancy that increase the pathogens spreading. Other interesting spray applications can be found in the study of waves, in food process and treatment as well as agricultural pesticides applications.

With such a wide ranges of application, it is understandable why the study of sprays and, in general, primary atomization and breakup have gained such popularity in the latest years, both from an experimental and computational sides. As optical devices and cameras improves, most of the applications mentioned above can be successfully measured and analyzed in great detail [5]. Furthermore, as the computers keep increasing their performances and High Performance Computing (HPC) becomes more and more affordable, numerical studies through Computational Fluid Dynamic (CFD) of sprays become more and more frequent, allowing significant insight of the phenomena. On the other hand, the range of applications is quite remarkable and the scientific community struggles to address some of the most cutting edge applications.

The study of sprays are usually cataloged using the dimensionless numbers typical of these flows:

$$
R e=\frac{u D}{\nu} ; \quad W e=\frac{\rho u^{2} D}{\sigma} ; O h=\frac{\sqrt{W e}}{R e},
$$

where $R e$ is the Reynolds number, We is the Weber number, $O h$ is the Ohnesorge number, $u$ is the flow characteristic velocity, $D$ is the characteristic length of the problem, $\rho, \nu$ are the density and the kinematic viscosity and $\sigma$ is the surface tension between the gas phase and the liquid phase. These numbers may refer to both the liquid phase and the gas phase (e.g. in case of air assisted atomizer), hence the parameter choice is crucial for characterizing the problem. The nature of this number is well known in literature and is at the basic of flow description both from macroscopic scale (e.g. when referred to the flow bulk) and for the microscopic scale (e.g. when referring to smaller scales of motion [6]). In general, in absence of gas flows that promote instabilities, the atomization regime can be characterized by two of these dimensionless numbers and their density ratio [7], although this approach has a limited range of applicability and relies on the development of turbulence in the nozzle (even if not fully developed). The atomization regimes are usually divided in the following categories (schematically represented in Figure 1.1):

1. Rayleigh regime: named after Lord Rayleigh and his studies [8], this regime occurs at low relative velocity. The small perturbations generated at the gas-liquid interface propagates until the droplets breakup is 


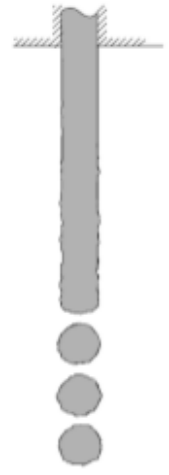

Rayleigh

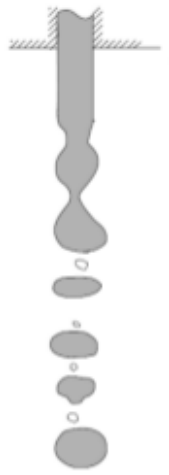

First

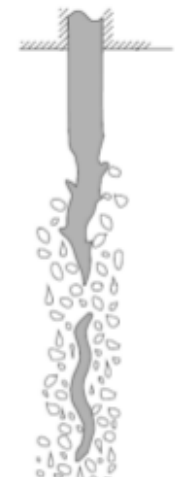

Second

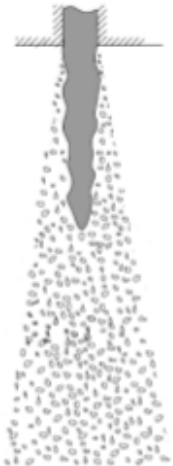

Incomplete atomization

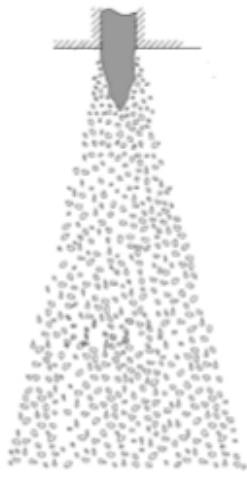

Complete atomization

Figure 1.1: Sketch of different atomization regimes.

reached. The description of this regime is quite complete in literature and addressed both numerically [9] and experimentally [10]. This regime is recognizable in dripping liquids, as the droplet formates mostly in the spray tip and presents a diameter comparable with the nozzle length.

2. First wind-induced regime: the relative velocity is higher and instabilities are increasing both their frequency and amplitude. The shear stress produced on the surface is now generating three-dimensional (nonaxisymmetric) wave propagations of the instabilities and the number of droplet generated increase. Despite that, the atomization is still occurring at the spray tip and presents large droplets.

3. Second wind-induced regime: The combination of surface instabilities and the increased shear forces generates a number of smaller droplets which are comparable to the perturbations frequencies. The atomization begins closer to the nozzle when compared to the previous two regimes.

4. Atomization: the aerodynamic forces exerted on the liquid surface are now predominant over the natural instabilities generated by the density gradients. The relative velocity is high enough to cause the breakup of most of the spray mass, although a certain amount of liquid, called intact core length keeps a connection with the liquid in the nozzle. Depending on the Re and $W e$ numbers, this length can reduce, although is always visible. 


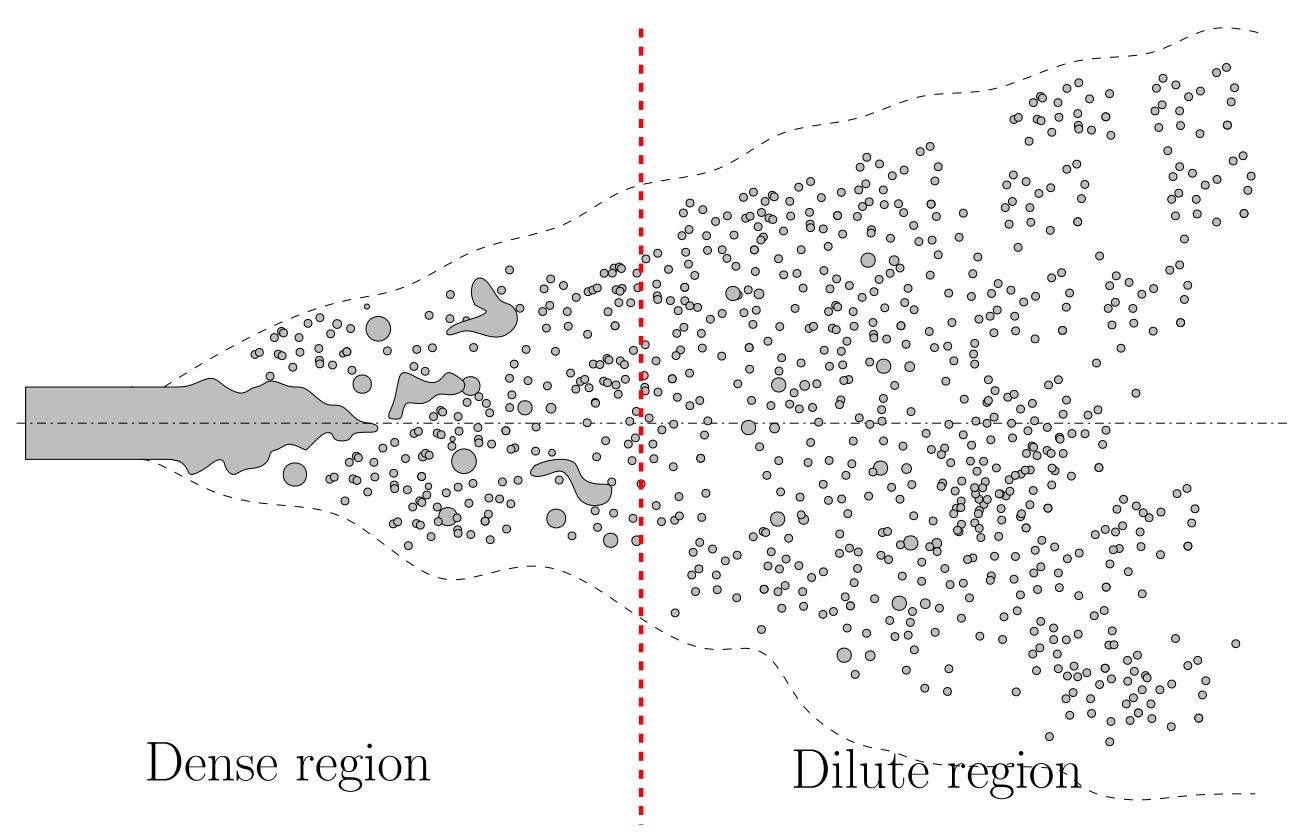

Figure 1.2: Sketch of a atomized spray main regions.

This last regime is the most popular among industrial applications, thanks to its capabilities of generating small droplets that promote mixing. The mechanisms that cause atomization are still unclear and only known qualitatively, especially in complex frameworks, as the one in Item 4. Lefebvre and Mcdonell [11] described these mechanisms, cataloged in the following categories:

- Aerodynamic instabilities: the perturbations generated on the liquid surface at the nozzle outlet get destabilized by the aerodynamic shear stress, caused by the difference in velocity between the liquid and the gas phase. The pressure gradients caused by this stress generates high frequency instabilities on the liquid surface, which prompt the formation of ligaments and the detachment of droplets. Instability mechanisms, such as Kelvin-Helmholtz, are among the effects of this velocity-induced shear, although a definitive explanation for the causality of this phenomena is still missing.

- Nozzle turbulence: according to Lefebvre and Mcdonell [11], this mechanism is one of the most important factors in generating atomization. In fact, the author suggests that this may explain why atomization 
still occurs in vacuum. The description of the turbulence features developed within the nozzle is not yet available, and its overall contribution to atomization is also difficult to quantify.

- Reorganization of the velocity profile: whether the flow is turbulent or not, a zero-velocity at the wall has to be displayed within the nozzle. Once the fluid outgoes the nozzle, the velocity profile "opens up", favoring the formation of radial components for the velocity, ultimately generating the instabilities that cause ligament formation and breakup, as described above.

- Cavitation: again, the influence of this mechanism on atomization is difficult to quantify, but numerical evidences show its importance. There are two different ways in which this phenomena may influence atomization. Firts, the formation of vapor separates intermittently the liquid flow from the wall, enhancing the overall turbulence within the nozzle. The implosion of bubbles also may forces the breakup or, at the very least, higher perturbations on the liquid surface. Ultimately a cavitating flow display a earlier breakup, a wider spray cone angle and a more prominent atomization.

A sketch of the atomized spray described in item 4 above, can be found in Figure 1.2. As the spray outgoes the nozzle and get exposed to high shear stresses, the breakup of liquid into droplets begin. At first, the breakup is extremely chaotic, large liquid structures can be found within the volume of the cone containing the spray and still a significant portion of the liquid is continuously connected to the liquid within the nozzle. At a certain axial distance (the spray axis is represented in dot-dashed line) the droplet distribution becomes more regular, larger droplet are rare and the average liquid volume fraction drops drastically (especially in the centerline). This distance marks the transition between the dense and the dilute region and it is often located at $25<x / D<30[6]$ where $\mathrm{x}$ is the axial position and $\mathrm{D}$ is the nozzle diameter (marked with a red line in Figure 1.2). When first atomized sprays were studied, this threshold was assumed to be similar to the region of gaseous jets where the flow was assumed to be self-similar $[6,12]$. This region, called the Near-Field region, was usually ignored in turbulence study made by means of hot-wire anemometry as limited comparability between different experiments was possible, therefore no generalization and study repetition about the jet velocity average and pulsating components were available. In this region turbulence is not yet fully developed while after $x / D>30$ most 
of flow local properties can be scaled with their respective centerline value, defining the self-similar region [6]. For sprays, a similar approach was used experimentally, but for different reasons which can be resumed in:

- High optical density: in order to understand the physics of the atomized region a sequential approach must be used from the moment in which the liquid leaves the injector until the moment when it becomes part of a droplet. Experimental techniques works well in dilute region but mostly fails in the dense [5]. This failure is due to the optical properties of the fluid in this region, which are characterized by a high absorbance given by the scatter produced by the liquid surface that impede the light to cross the medium. Techniques like Mie-Scattering and Schlieren fail in this region, while more modern techniques, such as optical connectivity [5, 13] and x-rays [14] are able to provide insight. On the other hand, this techniques are extremely expensive and requires difficult setups, therefore are quite uncommon.

- Typical length scales: atomized sprays requires significant velocities and long-enough nozzle to develop the wall turbulence and generate a sufficient shear stress able to induce atomization. This means high injection pressures and, usually, small nozzle, which contain the cost of the experimental facility. On the other hand, these conditions decrease both the length scale (requiring powerful microscopes) and the time scale (requiring fast cameras). The spatial and time resolution required are therefore a significant limitation.

These are still the main reasons for a lack of understanding of the physical phenomena that generates the dense region. Unfortunately, without this knowledge, it is impossible to create a complete physical description of the process. The usage of low detailed simulation like Reynolds-Average NavierStokes (RANS) and Large Eddy Simulations (LES) are incapable of predicting this transition and need to be coupled with experimental data in order to reach reliable results, useful for design and research purposes.

In this framework, Direct Numerical Simulation (DNS) is gaining popularity for both theoretical analysis and simple industrial problems. The improvement of HPC resources and numerical methods is increasing the capabilities of this methodology yearly [15] and can effectively been used to draw physical understanding of the atomization process. Although extremely expansive from a computational standpoint, DNS can directly reproduce atomization and resolve the velocity field up to the smallest scales of motion and therefore 
is perfectly suited for addressing the problem. Many doubts are currently been addressed by the research community on the actual limitation of DNS predictability $[15,16]$ but the constant increment of knowledge of sprays that is produced by DNS sudies is justifying the collective effort from the research community.

\section{$1.2 \quad$ Latest developments}

In this section, a review of the latest findings on spray dense region are presented. The presentation of these works will be divided between experimental and numerical.

A first introductory remarks should be made before proceeding further. As it will become evident over the next sections of the chapter, there is a substantial gap between experiments and simulations, due to the different approach that has been undertaken along the years. In fact, most of the experimental techniques that will be described in the following section have been developed with industrial applications in mind. A significant number of experiments for multiphase flows comparable with DNS studies of liquid-gas instabilities are available: for example Marmottant [17] in his thesis performs a interesting review of these experiments and his own scientific production $[18,19]$ is an elegant example of these works. On the other hand, industrial applications are usually targeting extremely high Reynolds/Weber numbers that from a simulation standpoint can only be addressed by RANS/LES simulations. A clear example of this trend is provided within the Engine Combustion Network (ECN) $[20,21]$ where industrial partners demands for technological readiness are substantially above the reach of DNS simulations.

This discrepancy in the application context of experiments and DNS simulations, contribute, along the years, to part ways between the two researchfield. Nowadays, it is well established in the scientific community the idea that, in order to produce scientific breakthroughs, a middle ground should be found in order to allow the study of these phenomena within a more complete framework produced by the combination of numerical and experimental investigations. This is especially true in the dense region, where virtually no knowledge is available.

With this goal in mind, the literature review on experiments and DNS simulations has two different objectives. On one hand, experiments that are quite popular and diffused in the sprays research community are discussed, with a focus of techniques capable of successfully addressing the study of the near field. On the other, the DNS studies performed in literature will be discussed 
with a focus on how far the research and analysis of these simulations has arrived so far, starting from very basic and fundamental studies of instabilities in sprays. This approach seemed the most suitable for this thesis as this work aims to get closer to the conditions used in the experimental techniques presented, while using methodologies commonly applied for conditions more suitable for theoretical studies.

\subsubsection{Experimental techniques in the dense region.}

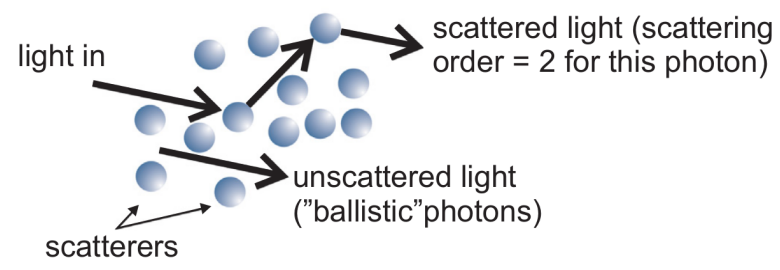

Figure 1.3: Sketch from Linne [5] of light scatter through opaque mediums.

As briefly discussed above, normal techniques used to characterize atomized sprays are ineffective in the dense region. This is due to the opacity of the region, quantified by the Optical Depth (OD), for which a detailed discussion about the optical basics can be found in [22]. In layman terms light travels straight until crosses a medium that scatters it. The more opaque a medium is, the more light will find scatterers obstacles and will be deviated. If the scattering is significant, some of the light beam can be refracted in directions that are unable to reach the optical objective (usually a high-speed camera) and less light intensity will be delivered. A sketch of this summary description (extracted from [5]) is provided in Figure 1.3. Usually, scattering order (number of scattering events experienced by a light beam) and OD are approximately of the same order. Here, the discussion will be limited to the categories in which OD can be divided in:

- Single scattering regime: it is characterized by $\mathrm{OD} \leq 1$, therefore most of the scattering events are of the order of 1 . There are numerical approximations and exact solution for these kind of optical regime.

- Intermediate scattering regime: here, $2 \leq \mathrm{OD} \leq 9$ and due to the complexity of such a regime, no mathematical solution is available. 
- Multiple scattering regime: $\mathrm{OD} \geq 10$ generates a diffused illumination as the significant number of scatter events lead to no predominant light direction.

Most atomizing sprays can be cataloged as medium scattering regimes, although a $\mathrm{OD} \geq 6$ is extremely common and sometimes also a multiple scattering regime can be displayed.

A number of methods along the years have been developed or adapted in order to study spray near field. The main are ballistic imaging, planar imaging, near-field microscopy imaging (or near-field visualization) and optical connectivity. Furthermore, when the light-source is based on x-rays, a number of other techniques can be applied, namely radiography, phase contrast imaging and scattering. A brief description of each method will be provided in the following subsections

\section{Ballistic imaging.}
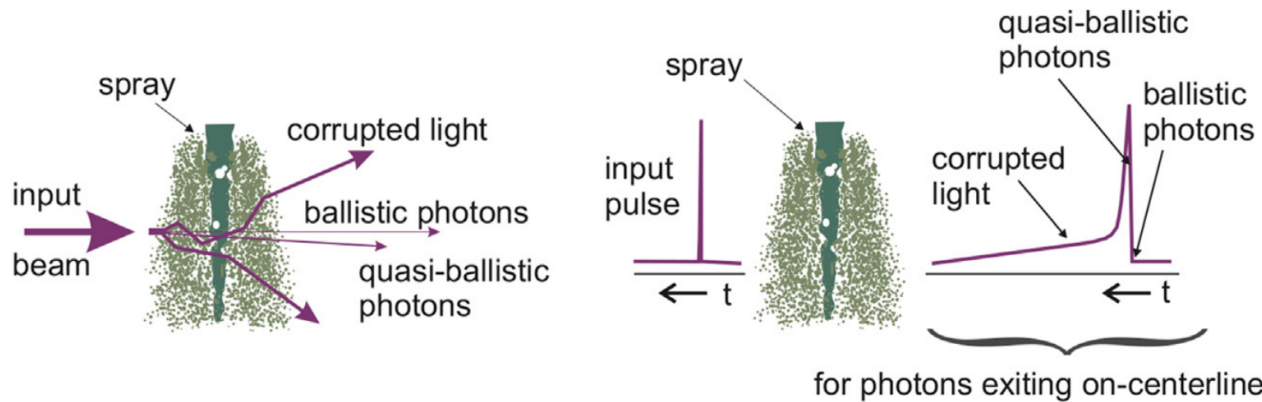

Figure 1.4: Sketch from Linne resuming the theory of ballistic imaging both from a geometrical standpoint (left) and a time-wise stand-point (right).

This technique relies on the use of a laser source on a spray side and a high speed camera on the other side to capture the shadowgram of intact liquid structures. The idea behind this technique, sketched in Figure 1.4, is to use the very few light beams that are crossing through the liquid without being heavily scattered by the droplets. If the light source is extremely powerful and the camera is extremely fast, black regions would mark the presence of liquid while every beam that will come out of a scattered pattern will be excluded by the image. The photons that cross through the liquid are called ballistic photons. As this is very difficult to realize physically, a certain amount of lightly scattered photons will be used to improve the image quality and they 
are called quasi-ballistic photons. An example of the results provided by this techniques for a fully atomized spray [23] is showed in Figure 1.5. Typical injection pressures for these kind of experiments ranges from 10bar [24], 20bar [25] and pressures up to 60 bar have been reported in literature [5], although the increasing OD at higher pressure forces the usage of a more expensive setup and result in a more diffuse image.
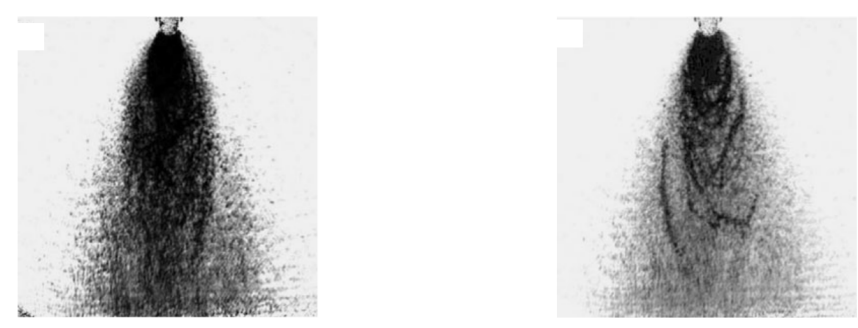

Figure 1.5: Data from Linne et al. [23] as an example of ballistic imaging results.

\section{Planar imaging.}

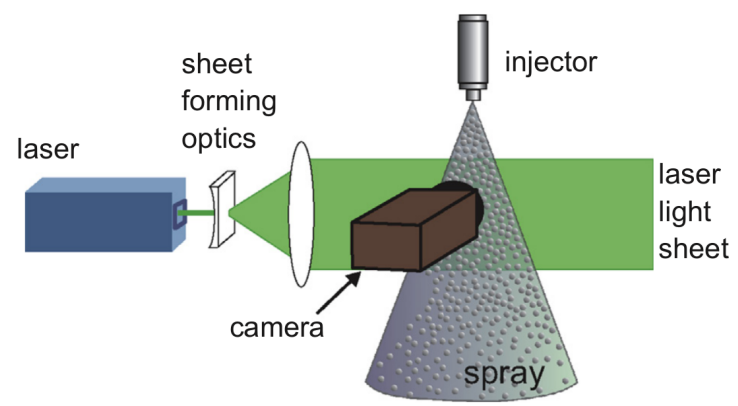

Figure 1.6: Sketch fo SLIPI technique setup from Linne [5].

This technique, also called "structured laser illumination planar imaging" (SLIPI) is one of the very few $2 \mathrm{D}$ techniques that are currently applied in dense region, in contrast with the dilute region where Particle Image Velocimetry (PIV) and derived techniques are often used. This technique is based on fluorescence, therefore the injected liquid is doped with a dye. The image obtained via fluorescence is compared to the Mie-scattering image of the same spray in order to extract only the light frequencies that are common to the two images. These allow to discard most multi-scattered photons, hence is 
especially useful in Multiple scattering regimes [26, 27]. Some pretty interesting sophistication of SLIPI can also allow to perform analysis of the Sauter Mean Diameter (SMD) [28], showed in Figure 1.7. It can be noted also that the results in Figure 1.7 show a certain polarization, which is typical of this technique. This phenomena is due to the physical scatter of the laser sheet while crossing the spray. In fact, it can be easily imagined that while the light beams (composing the laser sheet) go through the spray, a significant part of them will be heavily scattered, hence the technique will gradually lose definition across the spray. This is a well known limitation of this technique.

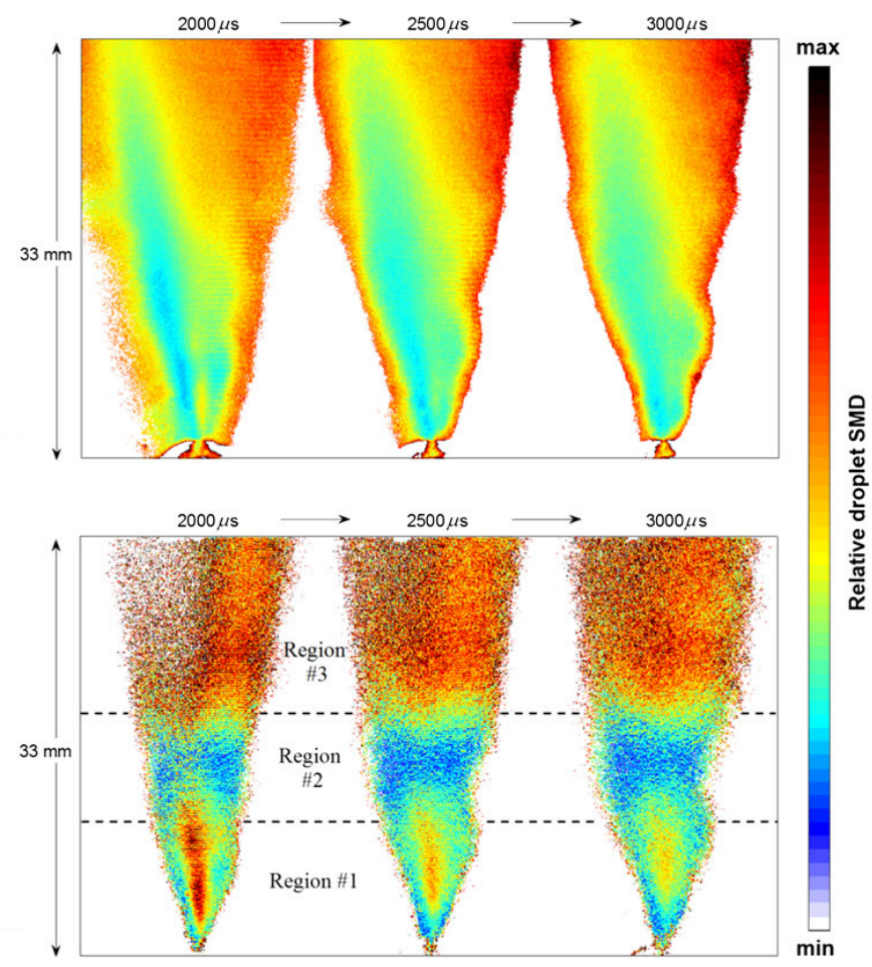

Figure 1.7: Sketch fo SLIPI technique setup from Berrocal et al. [28].

\section{Optical connectivity}

This technique is also fluorescence based and it has been developed by $\mathrm{G}$ et al. [13] and Charalampous et al. [30] for detecting the spray liquid core, which is usually the term used while referring to the amount of liquid that is still continuously connected to the liquid within the nozzle. The general idea is to use 


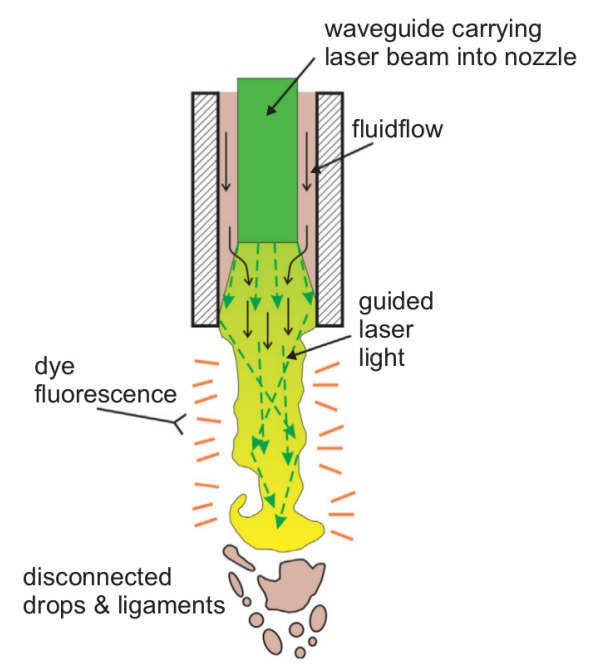

(a) Schematic representation of optical connectivity from [5].

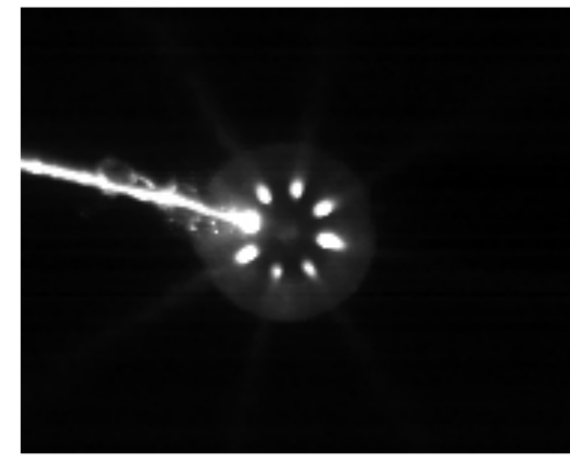

(b) Real application of optical connectivity in a multi-orifice automotive injector, from [29].

Figure 1.8: Realization of optical connectivity, schematic (left) and actual implementation in a real automotive multi-orifice nozzle.

a light-source (usually a optical fiber) within the nozzle and use the higher index of refraction of liquid to guide light as a preferential path then air. When the liquid breaks, the conduction of light stops, hence the detection of the liquid core is neat. Figure 1.8(a) shows a schematic of the optical fiber that needs to be installed within the nozzle. This type of experiment has the clear disadvantage of being extremely difficult to realize in real industrial applications for many reasons. In a first place, it often requires the modifications of the injector, as showed in Figure 1.8(b) for a multi-orifice automotive injector. This may modify the physical process (e.g. in the case of Figure 1.5 it may limit the plume-to-plume interaction) or modifying the turbulence generated within the nozzle. On the other hand, if an ad-hoc experiment can be designed it would provide an excellent way for validating DNS simulations, therefore it needs to be heavily accounted in theoretical studies of sprays. An example of the results provided by this technique is showed in Figure 1.9, where both shadowgraphy and optical connectivity experiments for the same sprays are showed and compared. 

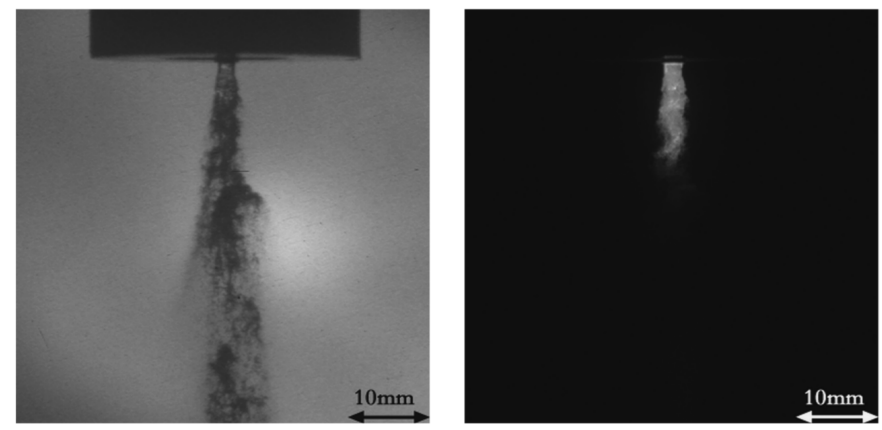

Figure 1.9: Example of results from optical connectivity experiments, from Charalampous et al. [30]. A shadowgraphy result is showed on the left, while its corresponding optical connectivity image is showed on the right.

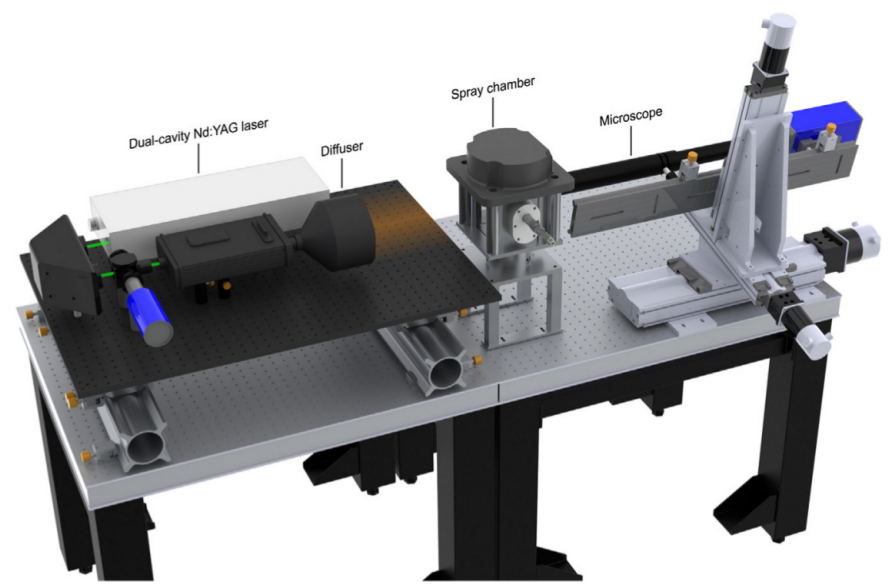

Figure 1.10: Render of the experimental setup for Near-Field microscopy, from Crua et al. [31].

\section{Near-field microscopy}

Near-field microscopy is an evolution of Diffused Backlight Illumination (DBI) [32]. The setup is extremely simple and relies on high quality (and expensive) components. It is composed by a high frequency pulsed laser (usually with a pulse shorter than $10 \mathrm{~ns}$ ), a diffuser that distribute the light source over a larger surface (sufficient for covering the application field) a microscope lens connected to the high-speed camera. The combination of the laser impulse power and duration with the shutter frequency of the camera are the real keys 
for applying this technique successfully. In fact the principle behind this nearfield microscopy is to generate an extremely powerful (hence concentrated in a short timespan) illumination and then capture the light passing through the spray (and obviously scattered) as neatly as possible. This can be achieved by means of a high-speed camera which need to have a high resolution (which are usually contradicting features).
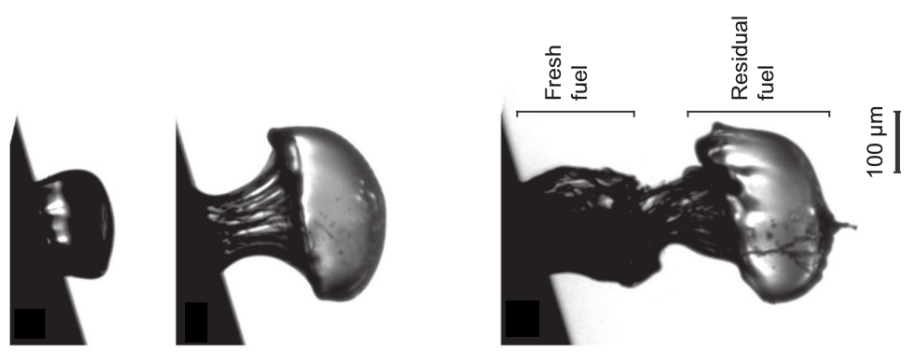

Figure 1.11: Initial transient of a diesel spray, from Crua et al. [31].

Some of the most notable results with this techniques have been achieved by Crua et al. [31]. This sample shows the clear potential of this technique for analyzing initial transients and compare them to numerical results. On the other hand, when the atomization becomes significant the scatter cannot be neglected and an important reduction in the light intensity rapidly decrease the usefulness of the experiment.

\section{X-ray phase constrast imaging}

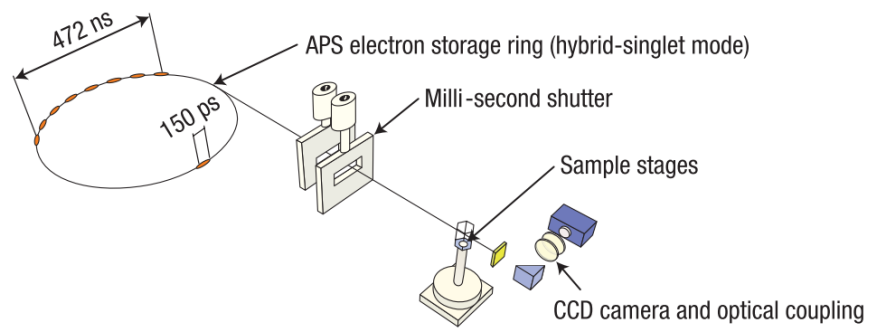

Figure 1.12: Sketch of the experimental layout used by Wang et al. [33] to perform $x$-ray PCI.

Phase Contrast Imaging (PCI) is a relatively simple technique, whose layout is similar to DBI previously discussed. The main goal is to obtain high 
resolution pictures of the dense region in order to improve the understanding of primary atomization. A schematic representation of the experimental layout used in PCI is depicted in Figure 1.12. The main feature of this technique relies on the usage of a synchrotron that circulates electrons in bunches in order to generate a pulse of approximately $150 \mathrm{ps}$ with a interval of $1.59 \mu \mathrm{s}$. This technology is likely the most expensive one needed in near-field visualization, hence only very few laboratories can afford to use it, one of the most notable (and developers of PCI) is Argonne National Lab, in the Advanced Photon Source (APS) division.

The main issue with this technique is how to treat the light signal before it is captured by the high speed camera. There are two main ways in which the refraction patterns, generated by the x-ray waves that cross the spray, can be sampled [5]:

- the "analyzer-based image" [34] in which a optical device (such as interferometry, diffractometry of in-line holography) is used to remove the background noise, such as performed in Schlieren techniques.

- the "propagation-based image" [35], the one mostly used at APS, which makes no use of optical devices in the light-path and allow the light to propagate in air in order to reduce the background light, such as in DBI. In this approach, some kind of data post-processing is required in order to achieve a clear image.

There is a significant literature, mostly developed recently, on how to treat the data collected using this technique [14, 37] and an interesting debate is still ongoing. Despite the approach used, this imaging technique shows a tremendous potential for the study of sprays as it can be showed from some results samples displayed in Figure 1.13. In Figure 1.13(a) (from Wang et al. [33]), PCI technique is used to analyze the spray transient (right picture) and it is compared with the analogous DBI for the same experiment where a laser source is used. It is evident how PCI allows a remarkable improvement in the determination of the ligaments formed behind the spray front and a higher definition in general. On the other hand, while invaluable for studying the physical phenomena, the comparison between the transient that can be achieved numerical and experimental techniques is limited for reasons that will become self-evident in this thesis. The type of experiment for which the comparison with numerical simulations looks very promising is showed, for example, in Figure 1.13(b), where stationary near-field imaging could be compared with DNS. 

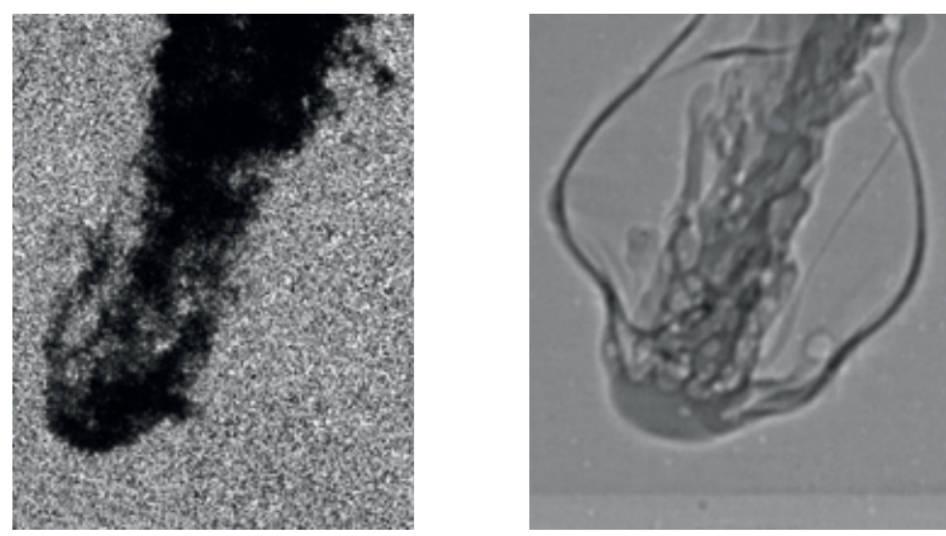

(a) Results from Wang et al. [33].

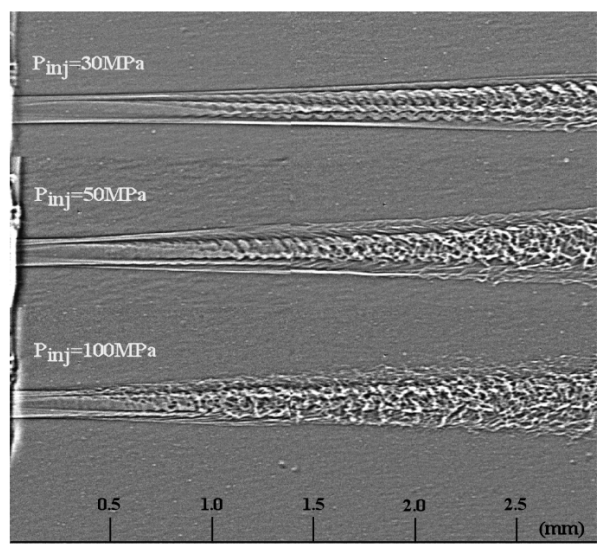

(b) Results from [36].

Figure 1.13: Results that can be produced by $x$-rays PCI.

\section{$\mathrm{X}$-ray radiography}

$\mathrm{X}$-ray radiography is, among the techniques discussed here, the one that has showed the highest diffusion in the CFD community thanks to its capability of measure the mass/area ratio, which is usually a significant parameter in CFD results (especially LES and RANS). The idea behind x-rays radiography (schematics in ) is to use a monochromatic beam across a spray, and rely on its absorption in order to reduce the amount of light received by an array detector. The array will produce a 2D plane where the intensity of the signal received is proportional to the density of the liquid and the amount of liquid the beam 


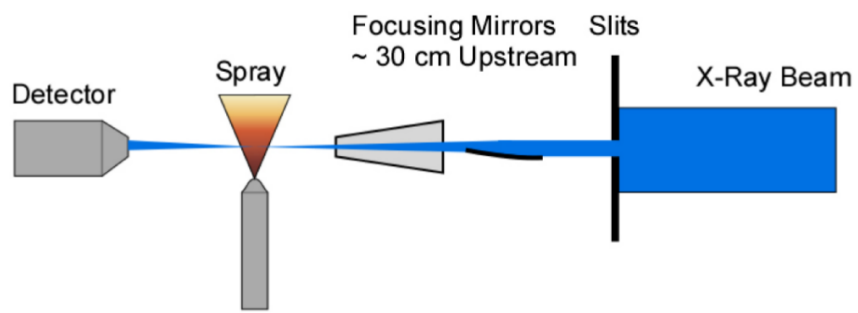

Figure 1.14: Sketch of the experimental layout used by Kastengren et al. [38] to perform $x$-ray radiography.

went through [14]. A useful upside of this technique is that scattering of $\mathrm{x}$-rays is almost negligible in most materials, hence attenuation is mostly achieved via direct absorption. If necessary, in order to increase the liquid absorption, the fuel can be doped [5]. This technique is capable of high resolution as the final result is actually provided by the average over many injection events. Furthermore, it can be used in conjunction with 3D tomography to get crosssection of density distribution even in very optical dense regions.

Figure 1.15 shows some of the results available in literature for x-ray radiography. Figure 1.15(a) shows the results produced by Kastengren et al. [38] of the projected mass over a spray longitudinal section. For whoever is familiar with results from RANS simulations or averaged LES and DNS simulations, this figure will certainly recall a familiar resemblance of mass-surface distribution. In Pickett et al. [32] a tomography of the cross sectional liquid volume fraction of a spray is computed and is here showed in Figure 1.15(b). Again, this type of results is extremely comparable with numerical results. The usage of special mathematical treatments of the data allows to compute also SMD [39].

\section{USAXS}

The Ultra-Small-Angle x-Ray Scattering (USAXS) is a rather new technique that allows to measure the projected surface area in a spray dense region. The way in which this method is applied is by directing a X-ray beam towards a spray dense region, as showed in Figure 1.16(a). The measured scattering intensity is than converted to an equivalent projected surface area by using the analysis package Irena, developed in [40]. The result is obtained by repeating the measurement at different axial position and the typical result is showed in Figure 1.16(b), from the work of Battistoni et al. [41]. The very interesting feature of this technique is the chance of combining it with X-ray radiography 

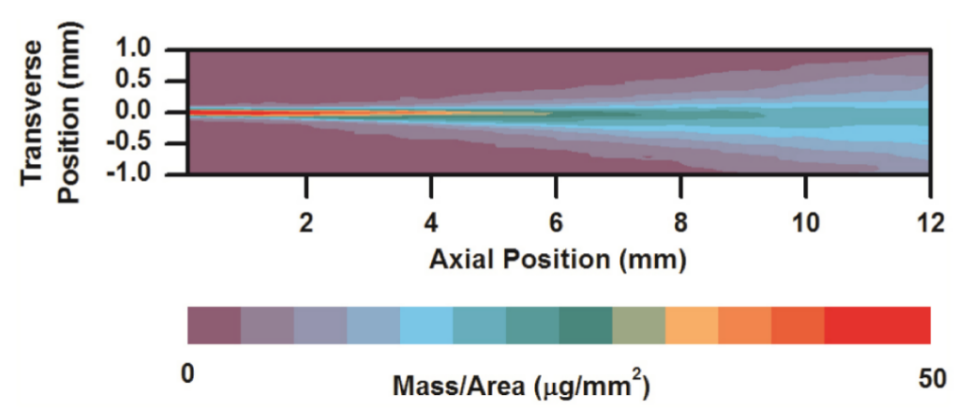

(a) Results from Kastengren et al. [38]
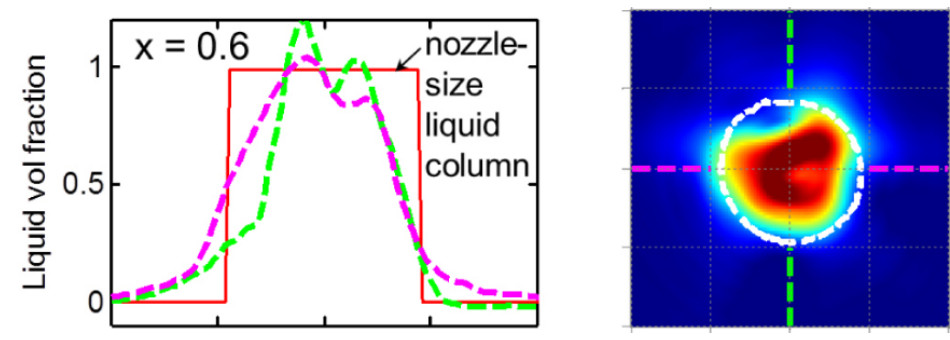

(b) Results from Pickett et al. [32]

Figure 1.15: Results of experiments performed using $x$-ray radiography

(which provides the projected volume) in order to obtain the droplets SMD in the section.

\subsubsection{DNS simulation of sprays in literature}

The usage of DNS for studying sprays has exponentially increased in the last decade. There is still no definitive framework for such a simulation, as depending of the specific application/objective it could differ vastly. Usually, the assumption of incompressible flow is made. While this assumption holds for $\mathrm{Ma}<0.3$ it is worth notice that usually DNS do not simulate excessively large Reynolds number due to the limitation posed by the needed computing resources. Starting with the numerical method used to reproduce the surface behavior, the two most common are Volume of Fluid (VOF) and Level-Set (LS). Both belong to the class of methods called interface-capturing methods, to be differentiated from the interface-tracking methods such as the FrontTracking. This methods, as well as their solution on a cartesian grid, will be described in Chapter 2. 


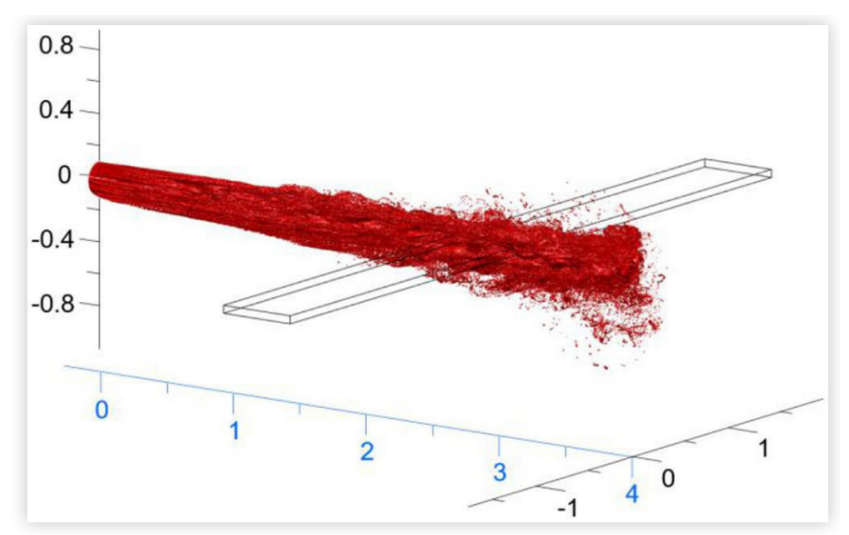

(a) Results from Battistoni et al. [41]

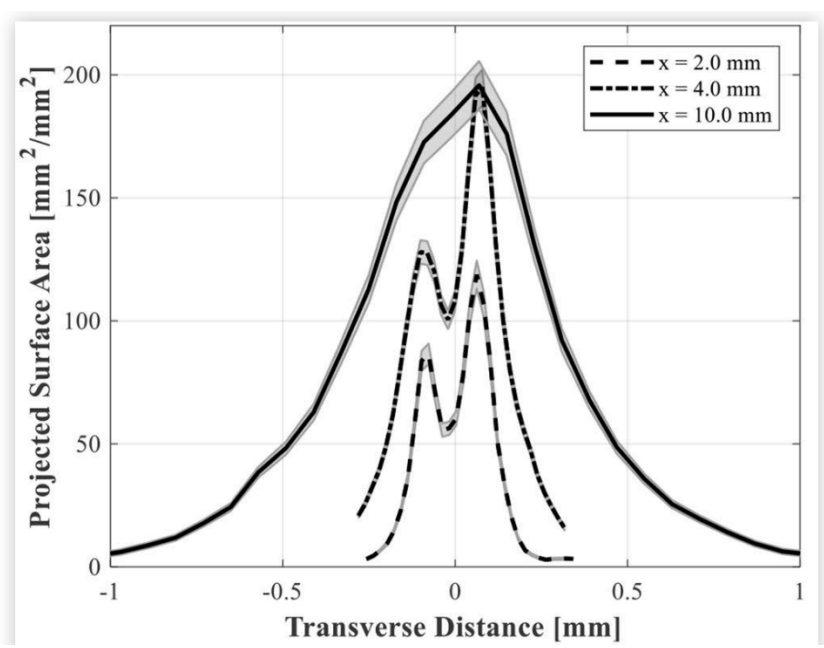

(b) Relative disposition of the $\mathrm{x}$-Ray beam in respect with the spray in USAXS technique. From Battistoni et al. [41]

Figure 1.16: Results from Battistoni et al. [41] of experiments performed using USAXS 


\section{Type of spray simulations}

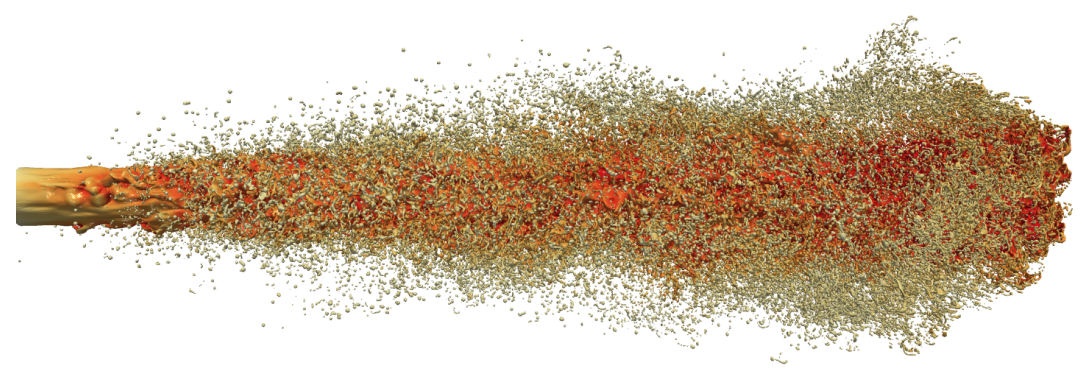

(a) Round spray.

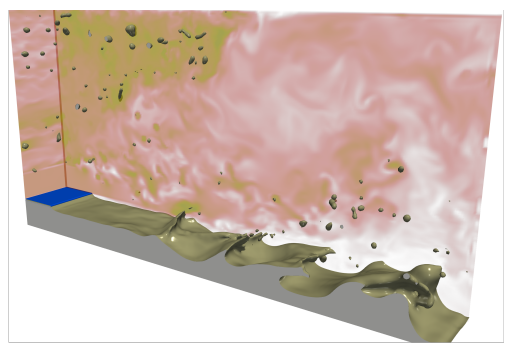

(b) 2D coaxial spray.

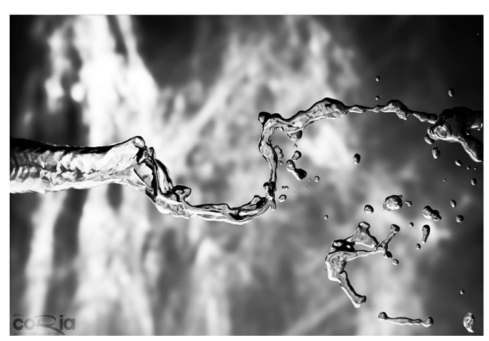

(c) Round coaxial spray.

Figure 1.17: Type of DNS simulations of sprays. Figure 1.17(c) is a render from [42].

There are various ways in which a simulation can be set and different geometries help assess different behaviors and reproduce different phenomena. Figure 1.17 shows the three different and most used type of simulation setups. Round sprays, showed in Figure 1.17(a) is one of the most common, as strictly related to plain nozzles, automotive nozzles, combustion chamber nozzle, medical sprays and so on. The focus on these simulation is usually posed on the inlet boundary conditions as they are one of the most important factor in creating the surface instabilities that trigger atomization; high injection speed (usually around $100 \mathrm{~m} / \mathrm{s}$ ) is then responsible for aerodynamic forces and consequent atomization regime, while the air is completely calm in the environment and none is injected into the domain. This case is the one 
that will be addressed in this thesis and the one to which a significant part of literature is devoted.

Other popular studies are about coaxial sprays, representative of airassisted atomizers. Examples of these simulation can be found in Figures $1.17(\mathrm{~b})$ and $1.17(\mathrm{c})$. Air assisted atomizers are extremely common in industrial applications. Field such as aeronautic and aerospace propulsions, pharmaceutical and food process are some of the fields in which these atomizers are applied. These applications relies on the possibility of injecting air that increase the instabilities of the liquid surface and force the breakup and have the significant advantage of requiring lower injection pressure for the liquid when compared to plain nozzles. The real applications are often composed by round nozzles [11], but $2 \mathrm{D}$ cases have some inherently advantages. A $2 \mathrm{D}$ setup, neglects partially the 3D helical effects that instabilities trigger on the spray core and that force the atomization to have a typical non-axisymmetric dynamic (e.g. see Figure 1.17(c)). Moreover, the simplicity of 2D approaches allows to ensure that some of the one-dimensional theory for sprays [10] are applicable and, in general, the simulation setup is inherently easier. On the other hand, while representative of only few industrial applications and sea waves, the $3 \mathrm{D}$ effects generated in this case have small relevancy to the $3 \mathrm{D}$ case which, for some studies, cannot be avoided. Both these setups generally have non turbulent $R e$ for liquid and turbulent $R e$ for gas (hereafter, $R e_{l}$ and $R e_{g}$ respectively) which makes the overall case turbulent thanks to the high vorticity field $\omega$, generated by the velocity discontinuities on the liquid surface.

Lower injection velocity are also one of the reasons why these cases are gaining a significant interest from a computational standpoint: in fact, DNS simulations of multiphase flows have significant limitations on the liquid velocity, being limited to Courant-Friedrichs-Lewy (CFL) numbers of about $0.2-0.4$. Furthermore, the need for resolving up to the smallest scales, namely the Kolmogorov scale $\eta[6]$, force the mesh size to be significantly smaller than RANS and LES simulations, as DNS of billions of cells [43] are not uncommon. These cases are also very well addressed in literature experimentally (e.g. [18]) and easy to study optically thanks to their reduced atomization, hence a perfect framework for studying theoretical aspects and validate simulation codes.

In the following section, the most significant simulations performed for the three configurations above will be discussed. Notably, as it is not usually addressed when talking about sprays, particle-laden flows will be neglected. On the other hand, a significant number of references about these kind of flows will be done in this thesis as a number of methods developed for those 
cases can be used to analyze and characterize sprays. Interesting review of particle-laden turbulent flow and dispersed flows can be found at [44, 45]. Other interesting cases are bubbly flows, which have a clear analogy due to their multiphase nature. These flow have an extensive characterization of their turbulent motion (e.g. [46]) and will only be addressed while analyzing the spray turbulent field.

\section{Recent spray simulation using DNS}

One of the very first contribution was provided by Ménard et al. [47] where a coupled LS/VOF ghost fluid method was used to simulate a diesel-like injection condition. In this work the nozzle turbulence was reproduced by using the synthetic turbulent inlet boundary condition (hereafter called SBC) from Klein et al. [48]. The turbulent integral length scale $L$ and the turbulence intensity $I$ were set according to literature data. The focus of this work was mainly on the numerical methods, but some interesting data about the axial penetration and mass concentration were provided. Moreover, in successive works such as $[49,50]$, performed by the same research group, this simulation was used to validate the results from an Eulerian-Lagrangian Spray Atomization (ELSA) model and to improve its efficiency by analyzing the liquid-gas surface. The same research group continued the analysis of sprays by undertaking a very interesting approach: from the simulation performed by Lebas et al. [49], Canu et al. [51] extracted specific regions of fluid where different mass concentration where available. This process, reported in Figure 1.18, allowed to select specific conditions to be simulated in periodic boxes with homogeneous-isotropic turbulence. This approach has the inherent advantage of reducing the computational resources required for simulating the interaction of liquid surface curvature (a parameter closely related with the breakup due to the surface tension) with turbulence, here resolved up to the Kolmogorov scale. The intended knowledge that can be gathered from here aims at improving current simpler models like RANS or LES-ELSA but without a local characterization of the turbulence in the extracted regions this information may just be partial as, due to the strong advection of the main flow, turbulence may behave differently than in box-turbulence, for reasons that will be explained in Chapter 6.

A substantial contribution was provided by Shinjo and Umemura [52], where the largest DNS simulation (until recent developments) was performed. For reasons that will become apparent in Chapters 6 and 7, these are the only known simulation of round sprays where the Kolmogorov scale was likely reached. On the other hand, the setup of these simulations was rather simplis- 


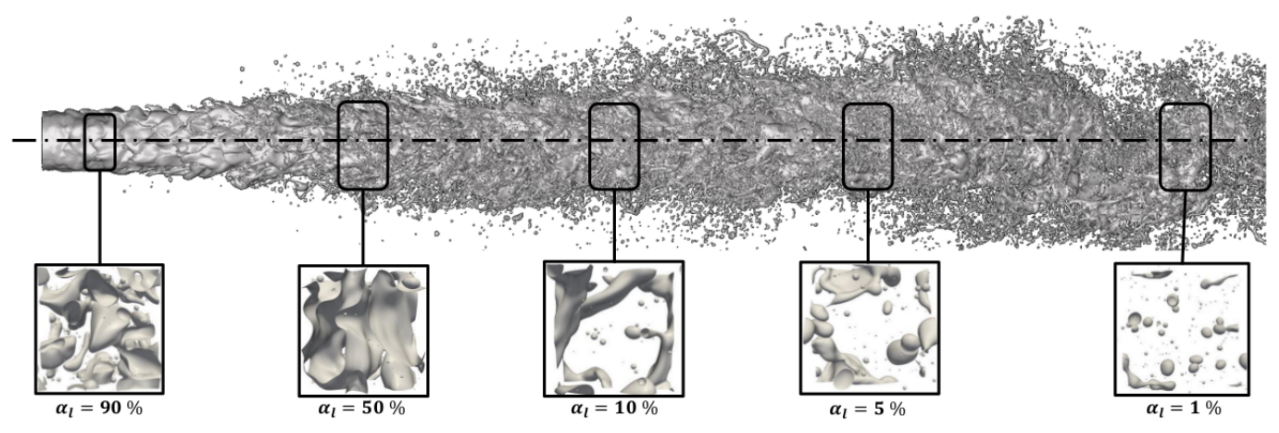

Figure 1.18: Results from Canu et al. [51].Selection of ad-hoc region of different mass concentration (here described by means of the curvature distribution).

tic as no inflow boundary conditions was used. This limited the atomization and reduced the computational load, allowing to resolve up to 6 billion cells. Despite the simple approach, some interesting results were harvested. In this paper, the same authors analyzed the effect of mesh size on artificial droplets, concluding that at least four cells per diameter in each direction were needed in order to consider a droplet as such (criteria not followed in most of literature nor in the present thesis). In the same work, an interesting analysis of the perturbations on the liquid surface and the formation of ligaments was performed. In [43], the same authors analyzed the dynamic of the spray front, studying the formation of the toroid vortex behind it and quantifying the vorticity and recirculation caused by it. Finally, in [53] the surface instabilities were analyzed based on both a $2 \mathrm{D}$ and a $3 \mathrm{D}$ approach, highlighting how the formation of hairpins vortexes in the recirculation region cause the creation and propagation of disturbances on the liquid core of the spray. These perturbations, incapable of climbing up the spray core, are then dumped by the surface tension and would need significant higher aerodynamic shear to be stabilized. These works pioneered this approach inspiring many more similar works during the last decade. Furthermore, the knowledge and data gathered in the four simulations performed were used to propose a new mathematical formulation of a Sub-Grid Scales (SGS) model for LES simulation that was first theorized mathematically in [54] and finally implemented in [55].

Despite the enormous amount of published work that was inspired by the work of Shinjo and Umemura, the applicability of these results was limited far many reasons. First and foremost, the most significant flaw of these works 


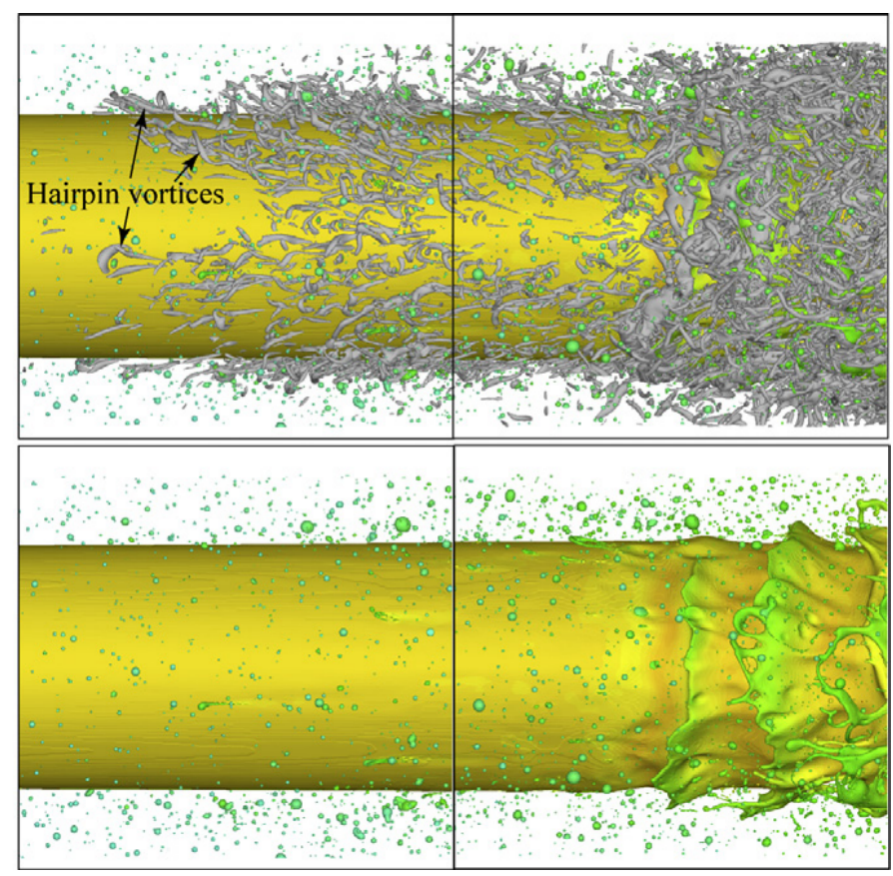

Figure 1.19: Results from Shinjo and Umemura [53]. The top figure shows how the turbulent structures propagates and decay upstream the spray core, while the lower one shows the plain render of the liquid core.

is that only the transient phase of the spray penetration is addressed. In fact, the lack of perturbations stability cause the instabilities to be dumped quickly, as showed in Figure 1.19. While this allows the authors to draw interesting and fundamental considerations there is no way of validating this calculations. Reproducing exactly this test-case experimentally is impossible as no validation can be achieved. DNS calculation can in fact be validated by analyzing their spectral behavior or by reproducing an experiment and both ways are unfeasible for this case. More details on general DNS validation will be discussed later in this chapter.

One of the very first works about the study of span-wise and stream-wise instabilities was performed by Lozano et al. [58]. Here the authors used a vortex dynamic code for the interface tracking of a liquid sheet with a relative velocity with respect to air, studying the formation of ligaments due to the stream-wise instabilities formed. The same approach was lately revisited for plane and round jets, respectively from Jarrahbashi and Sirignano [56] and from Zandian et al. [57], as part of the same research group. In this approach, 


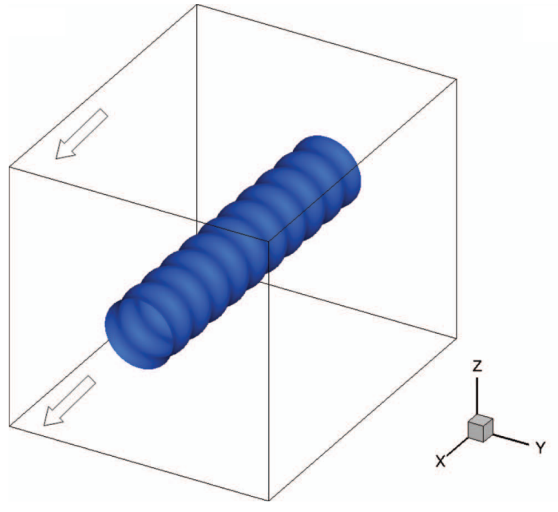

(a) 3D Round jet from [56]

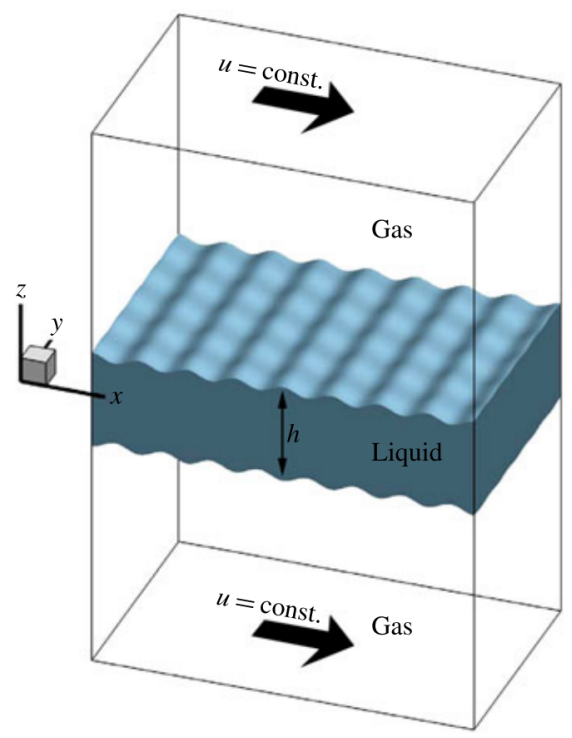

(b) 2D plane spray from [57]

Figure 1.20: Type of DNS simulations of sprays. Figure 1.17(c) is a render from [42].

a flux of air is flowing around a jet in a periodic box. While the reasoning behind such an approach can be less intuitive than that proposed by Shinjo and Umemura [43], it is undoubtedly effective. This approach neglect the initial transient (which in most simulations accounts for a significant percentage of the overall calculations) and all the related instabilities, while quantifying only the aerodynamic interaction between the two fluids. Such a framework allows to highlight only the instabilities effects and to isolate the ligament/droplet generation given by this aerodynamic shear. The simplicity of the setup allows to perform a significant number of simulation, which allows to sweep over a significant number of $R e, W e$ and liquid/gas density ratio. The round jet cases $[56,59]$ found that the formation of counter-rotating vortexes and hairpin-like structures is at the base of the lobe formation, which then will evolve into ligaments and finally into droplets. eventually, after a sufficient time is given to the process to complete, the atomization becomes more and more chaotic and helical effects are no longer negligible as the turbulence field goes towards the statistical stationary condition. The plane jets $[57,60]$ allow to reduce the uncertainties given by these configurations and maintain a significantly simpler setup on which perform the parameterization study. 
In the recent works proposed by Ling et al. [61] a more detailed approach was used to characterize the turbulence feature within the flow. In this case, the simulation modeled a 2D coaxial spray, such as the one in Figure 1.17(b) or the one proposed by the authors themselves in [62]. In this first phase, the authors discussed how the liquid sheet creates and how the convergence on the droplet size distribution can be achieved once the mesh is sufficiently fine. Furthermore, a first attempt of analyzing the simulation convergence thanks to the kinetic energy was proposed. This first step in analyzing the turbulent field was further addressed in [61], where more advanced statistic of turbulence were provided. Aside from an interesting result regarding the energy balance along the transverse direction, they also pioneered the study of Kolmogorov scale and Batchelor scale based on the resulting energy dissipation. While their simulations are properly resolved, according to their estimations, these values have been obtained mainly for the gas phase, in order to avoid the discontinuities produced by the mobile liquid surface. Furthermore, the difficulties in estimating the fluid properties are significant when in presence of an interface and their method is an effective workaround. On the other hand, this thesis will discuss deeply this aspect in further chapters as one of the key points discussed in this work. These work constitute a first and very significant study of the turbulent field produced during the atomization regime. The same authors have addressed in many occasions round sprays, most notably in [63] where the effectiveness of the Lagrangian Point Particle (LPP) was proven to be comparable to an Eulerian approach. This research group also authored the open-source code PArallel Robust Interface Simulator (PARIS), also known as ParisSimulator, that will be used in this thesis to perform DNS simulation.

DNS has also been extensively used to tackle industrial applications, as well by Desjardins et al. [9]. In this work, the researchers showed how knowledge of extremely complex flows, such as pressure swirl atomizers, can be achieved by using LES and DNS simulations. Both in this work and in previous studies, such as Pitsch and Desjardins [16], the authors addressed simpler geometries, such as the round spray [64] and the planar jet (similar to Lozano et al. [58]). In [16], the authors characterized the evolution of the liquid sheet thickness by means of its volume fraction and root mean squared (rms) value. Also, the author, after describing the evolution of the one point statistics for the flow, made a significant first attempt in computing the energy spectra along the streamwise direction, demonstrated a clean cascade and a $-5 / 3$ law [6].

Boundary conditions have proven significant influence in many studies. For instance, "On simulating primary atomization using the refined level set grid method" [65] and Desjardins et al. [64] showed that by using a Mapped 


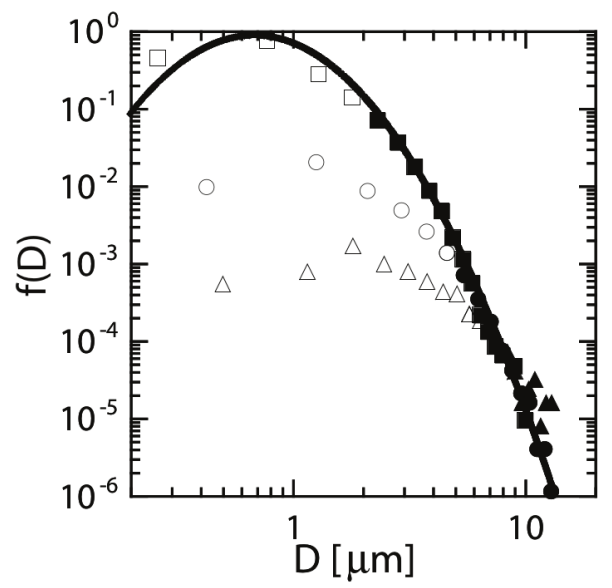

Figure 1.21: Results from "On simulating primary atomization using the refined level set grid method" [65]. Different data sets have different symbols for different RLSG grid-size, with a standard grid of size $\Delta x=1.56 \mu \mathrm{m}$. The $R G L S$ values are: triangles $1.56 \mu \mathrm{m}$, circles $0.78 \mu \mathrm{m}$ and squares $0.39 \mu \mathrm{m}$.

inflow Boundary Condition (hereafter MBC) a significant increment of the atomization regime can be achieved. In particular, the former used a different grid for the solution of the LS (here called Refined Level Set Grid, RLSG) method and for the solution of the Navier-Stokes (NS) equation. By fixing the "kinematic grid", the amount of turbulent energy that can be computed is fixed, while by varying the "surface grid" (RLSG) an interesting quantification of the effects of those velocity scales over the total atomization can be achieved (of course if assuming that smaller size liquid structures are not affecting significantly the turbulent field). The results in Figure 1.21 showed that for finer RLSG more atomization can be achieved and the peak of the log-normal distribution of droplets moves towards smaller diameters.

\section{Considerations over the scales of motion and atomization}

The works presented until this point mostly agree that a full solution for both the atomization scales and the turbulent scales is far from been reached. We define now the three main scales that should be addressed when talking about multiphase turbulence:

$$
\eta=\left(\frac{\nu^{3}}{\epsilon}\right)^{\frac{1}{4}}
$$




$$
\begin{gathered}
\eta_{H}=C_{h}\left(\frac{\sigma}{\rho_{g}}\right)^{\frac{3}{5}}\left(\frac{\epsilon}{\rho_{g}}\right)^{-\frac{2}{5}} ; \\
\eta_{B}=\left(\frac{\nu \beta^{2}}{\epsilon}\right)^{\frac{1}{4}} ;
\end{gathered}
$$

where $\eta$ is the Kolmogorov scale, $\eta_{H}$ is the Hinze scale, $\eta_{B}$ is the Batchelor scale, $\epsilon$ is the turbulent kinetic energy dissipation rate, $\beta$ is the mass diffusivity and $C_{h}$ is the Hinze constant, usually assumed to be $\approx 0.725$ [61]. The Kolmogorov scale defines the size of the smallest eddies that characterize a turbulent flow [66]; the Hinze scale defines the size of the droplets that can be developed within a turbulent flow [67]; finally, the Batchelor scale is an analogous to the Kolmogorov scale in case of diffusion of a scalar field (in this case mass) and should be universally applicable to both scalar fluctuations and turbulent fluctuations [68]. The discussions about whether or not each scale applies to each case are vastly spread in literature as many uncertainties surrounds the definitions of $\epsilon$ and $\nu$ in multiphase flows. The complexity of multiphase flows is such that authors often prefer avoid the complexity of the flow in the mixing layer and evaluate properties just in one region, usually the gaseous one [61]. Consequently, this approach is often miss-predicting flow features, such as overestimations of $\eta_{H}$ in respect to the smallest droplet detected in the simulation. While in that work the author suggests that turbulence may not be the main driver for droplet formation (hence the discrepancy between $\eta_{H}$ and the droplet size) this seem to be in disagreement with most of the findings in this thesis.

Other authors $([15,16])$ asserts that a complete numerical solution of a multiphase turbulent flow is likely to be unreachable as the solution of the interface thickness is unresolvable and of order of magnitudes below the Kolmogorov scale. This argument, while based on a solid observation of the surface curvature and tension role in primary atomization, is also quite unpractical for simulation design purposes, as suggests that when the Kolmogorov scale is resolved, everything behind it have less and marginal impact over the overall flow.

\subsection{Objective}

The whole discussion carried in previous sections aimed to highlight the main progresses made in experiments and DNS simulations within the last 20/30 
years. Aside from that, it is clearly showed within the review that significant discrepancies can be detected between the two approaches. While the experimental techniques have heavily pursued the industrial applications, DNS has investigated basic phenomena and therefore kept a significant distance from physical conditions that may overlap many effects, making it difficult to extract result-oriented informations and prove theories. This distance has somehow made extremely difficult the conjunction of the two worlds, as each one has developed significant knowhow and expertise in solving specific issues, unrelated between experiments and DNS. For example in the automotive sector, while a significant effort in experiments has been devoted in reaching cutting-edge injection pressures, multi-injection strategies and significant counter pressure, DNS struggle to reach injection velocity of $100 \mathrm{~m} / \mathrm{s}$, which are close to 1970s indirect diesel injection and far from being significant in nowadays industry. In the aerospace sector, experiments have moved towards testing complex geometries and flows that provide significant difficulties in the numerical setup.

Despite its reduced capabilities in terms of simulating real industrial applications, DNS is uniquely suited to generate physical knowledge and theories, thanks to its capability of providing extensive information on both velocity and scalar fields. The complete resolutions of all the scales of motions allows to correlate directly turbulence and atomization processes, and set the framework for causality analysis. On the other hand, handling this information can be a monumental task. DNS can generate a significant amount of data, up to tens of TB per simulation and a clear methodology for the analysis phase must be established often before the simulations is run. The development of effective post-processing strategies is key in extracting knowledge from DNS simulations, with post processing procedure sometimes heavier than the calculation itself. For example, the computation of high-order statistical moments, two-point statistics and high-resolution spatial gradients may impose memory requirements that limits significantly the methodology applicability.

Once the DNS data post-process goal is set, the simulation can be modified to realize on-the-fly statistics but this requires a significant a-priori knowledge of the physical problem. In spray dense region, this knowledge is not available especially from a kinematic standpoint. The main reasons for it are summarized in the following:

- Lack of experimental velocity data in the dense region: from what discussed above, the main aim of the experimental techniques is harvesting information on morphology and spray cone angle formations. 
While some information of the mean axial velocity could be gathered from the spray front penetration, this is insufficient for having comparable data that may help validate or design calculations. Moreover, typical experimental techniques for velocity measurements like hot-wire, although applied in disperse multiphase flows, are rare in sprays and, to the best of the author knowledge, no significant publications on the topic are available.

- Lack of detailed analysis of the dense region turbulent field: while many DNS studies have been performed in sprays, no significant data on the turbulence was published by the beginning of this thesis. This would have helped establishing mesh-size from a kinematic standpoint, satisfying the Kolmorogov scale requirements. On the other hand, for reasons that may be found in [43], a serious argument can be made that the integral length scale measured in the statistical stationary turbulence is not the most relevant scale for simulation design. In fact, the main vortex is the one created behind spray tip, which is a typical transient phenomena. Hence, also a detailed analysis of this eddy is required, as it will influence the simulation domain size, the analysis of third and fourth statistical moments (capable of detecting fluctuations characterized by large turbulent structures) and the time interval over which perform the statistical study.

- Lack of detailed knowledge of the nozzle flow turbulence: as extensively proved in the DNS literature proposed above ([43, 47, 56, 69, 70] among the others discussed) the surface instabilities generated by the inflow turbulence are directly responsible for the dynamics with which the atomization process takes place. Nevertheless, there is a substantial lack of knowledge on what a realistic inflow boundary condition should produce in terms of nozzle turbulence features, namely turbulence intensity and lengthscale. This misinformation has various reasons. In a first place, real nozzle turbulence (e.g. medical devices and automotive injectors) are well-known to produce non-developed turbulent profiles $[71,72]$. Turbulence transition is always present in turbulent flows, but its origin is still partially unknown, therefore its characterization has not been generalized [73-75]. This means that no theoretical approach can be applied in imposing inflow boundary conditions and simplification needs to be made. For this reason, the topic is often avoided in DNS literature and light discussions on it are provided. Finally, most authors prefer to just discuss the topic briefly due to the difficulties in numerical comparisons required for reinforcing results validity. 
- Insufficient resolution for multiphase flows: as discussed by [16, 69] the mesh resolution needed for atomizing multiphase flows may be of the order of smallest surface thickness, hence its exact numerical solutions is likely unachievable also with exascale computing. Moreover, from an experimental standpoint, this resolution is in the order of nanometers, which is potentially the size of the smallest satellite droplets generated within this process [76]. To the best of the author knowledge, no experimental techniques is up to this task. This limitations in resolutions, both numerically and experimentally, makes it significant more challenging the comparison between experiments and DNS, due to the increase of error in numerical method. Ideally, the best comparison could be achieved by comparing droplet distributions such as the one showed in Figure 1.21 against experimental data. That said, such a resolution may be not fully necessary as for the theory developed by Kolmogorov [66], Gorokhovski and Saveliev [77], and Kolmogorov [78] the information missing may be superfluous for designing correct physical description and interpretation of the process.

The whole scenario depicted until this point is at the origin of the research plan designed for this thesis. The focus of the work is based on the three pillars that have been identified until this point, namely the morphological analysis, the granulometry analysis and the turbulence field analysis.

- Morphology analysis is generally seen as a qualitative analysis of the spray. It tends to catalog surface instabilities, liquid structure features and atomized field in a fashion helpful for drawing physical assessments and conclusions. In this work, the approach proposed by Jarrahbashi and Sirignano [56] and Zandian et al. [57] is employed to reach behind quality assessments and try to define quantitative conclusions based on the analysis of the vorticity field. Furthermore, the study of the spray tip morphology is somehow closely related to the simulation domain. As it will be explained, the spray front size determines the radius of the toroid defining the largest eddy in the domain and therefore the largest event/flow feature. This information is necessary in order to estimate the domain size. Furthermore, spray morphology is also able to partially reveal whether the domain is sufficiently large for reaching the self similar region, which is also an important condition for allowing comparison with experimental techniques.

- The droplet analysis will be a fundamental aspect of this thesis, but its aim needs to be clarified. In a first place, the liquid structures will be 
analyzed in post processing, while the general approach, derived from a Lagrangian-Point-Particle, usually performs the analysis within the simulation run. Furthermore, the analysis performed aims to be a more discrete approach, where each droplet is considered, regardless of its shape, as an individual entity with its own properties. This approach also resemble the original fractal theory developed by Kolmogorov [78] for solid carbon particles and extended to droplets by Gorokhovski and Saveliev [77], which provided interesting theories that should be addressed in the future [15]. Ultimately, the analysis in this thesis aims to find droplet size and location distributions, as well as topological patterns and formation description. The main goal here is to produce a significant amount of data that may create a bridge between experiments and simulations. In fact, droplet diameter distribution in the dense region, showed by means of Probability Density Function (PDF), could be comparable with experimental data of the dilute region if the dynamic with which droplets are generated is understood (hence enabling to remove distribution bias given by the dense region). Furthermore, a topological analysis can give significant informations on interaction between droplet and turbulence (widely addressed in particle laden flows [79]) and provide hints of significant flow region in which apply high resolution experimental techniques that can be compared with numerical results.

- Finally, the analysis of the turbulent field is one of the main contribution of this thesis. There is a significant computational gap between single phase flows and multiphase flows due to the lack of theoretical comprehensive knowledge of turbulence and insufficient experimental technique for the latest. While single phase flows DNS simulations are often designed using a given knowledge of Kolmogorov scale, the multiphase ones are usually performed under significant uncertainties of the turbulence feature. This issue helped forging the rephrasing of DNS for multiphase flows into Detailed Numerical Simulation [16] as a more suited term to really explain what can be achieved with DNS. The lack of available comparisons for quantifiable kinetic variables in multiphase DNS is therefore coming from both experiments and turbulence theory, partially discrediting the capabilities of DNS to predict the real flow behavior. For these reason, this thesis aims to improve the knowledge of turbulence in multiphase flows, by two main analysis. At first, the study of a methodology for determining the Kolmogorov scale is addressed and compared with an a-priory calculation. Finally, the derivation of a turbulence spectra will be addressed and the same will be used as a quantifying tool to study the atomization patterns. 
While performing these analysis, the issue of inflow boundary conditions is extensively addressed. The first part of the analysis will be about defining the effects of turbulent integral quantities, such as turbulence intensity and lengthscale in the method presented by Klein et al. [48], over the primary atomization. While doing so, the aim will be defining the optimal simulation domain for harvesting turbulence statistics and determine de influence of those parameters via morphological and granulometric analysis. Finally, the type of structures (isotropic/anisotropic) influence over primary atomization will be performed and turbulence data will be collected, analyzed and extensively used as a comparison tool in order to justify differences. In this case, the synthetic inflow boundary condition in [48] and a mapped inflow boundary condition (as in [69]) will be used and their implementation will be extensively discussed.

Overall, the objective of the whole research can be summarized in defining a methodology for performing and analyzing DNS of spray in the dense region. In other words:

- Provide tools and data for simulation design: defining how to design the domain shape, size and mesh size that are able to provide significant informations of both turbulence and atomization on a "affordable" computational price. This means, among other things, performing a novel characterization of the turbulence in the dense region. Finally, explaining pros and cons of type of inflow boundary conditions in order to allow the choice of an ideal setup depending of the simulation target.

- Provide tools for simulation analysis: the three analysis pillars described above will be fully explained and their application will be used to display their capability for comparison and theoretical analysis

\section{References}

[1] Sirignano, W. A. and Mehring, C. "Review of theory of distortion and disintegration of liquid streams". In: Progress in Energy and Combustion Science 26.4 (2000), pp. 609-655. DOI: 10.1016/S0360-1285(00) 00014-9.

[2] Lasheras, J. C. and Hopfinger, E. J. "Liquid Jet instability and Atomization in a Coaxial Gas Stream". In: Annu. Rev. Fluid Mech. 32.1 (2000), pp. 275-308. DOI: 10.1146/annurev.fluid.32.1.275. 
[3] Ziaee, Ahmad et al. "Spray drying of pharmaceuticals and biopharmaceuticals: Critical parameters and experimental process optimization approaches". In: European Journal of Pharmaceutical Sciences 127.November (2019), pp. 300-318. DOI: 10 . 1016/j . ejps . 2018 . 10.026 .

[4] Bourouiba, Lydia, Dehandschoewercker, Eline, and Bush, John W.M. "Violent expiratory events: On coughing and sneezing". In: Journal of Fluid Mechanics 745 (2014), pp. 537-563. DOI: 10.1017/jfm.2014.88.

[5] Linne, Mark. "Imaging in the optically dense regions of a spray: A review of developing techniques". In: Progress in Energy and Combustion Science 39.5 (2013), pp. 403-440. DOI: 10.1016/j.pecs.2013.06.001.

[6] Pope, Stephen B. Turbulent Flows. 2001. DOI: 10.1088/0957-0233/ 12/11/705.

[7] Reitz, R. D. and Bracco, F. V. "Mechanism of atomization of a liquid jet". In: Physics of Fluids 25.10 (1982), pp. 1730-1742. DOI: 10.1063/ 1.863650 .

[8] Rayleigh, Lord. "On the instability of jets". In: Proceedings of the London Mathematical Society s1-10.1 (1878), pp. 4-13. DOI: 10.1112/ $\mathrm{plms} / \mathrm{s} 1-10.1 .4$.

[9] Desjardins, Olivier, McCaslin, Jeremy, Owkes, Mark, and Brady, Peter. "Direct Numerical and Large-Eddy Simulation of Primary Atomization in Complex Geometries". In: Atomization and Sprays 23.11 (2013), pp. 1001-1048. DOI: 10.1615/AtomizSpr. 2013007679.

[10] Ashgriz, Nasser. Handbook of Atomization and Sprays. Springer Science \& Business Media, 2011. DOI: 10.1007/978-1-4419-7264-4.

[11] Lefebvre, A. and Mcdonell, Vincent. Atomization and Sprays, Second Edition. CRC Press, 2017, p. 300. DOI: 10 . 1016/0009-2509(90) 87140-N.

[12] Corrsin. Spectra and Diffusion in a Round Turbulent Jet. Tech. rep. 8. 1943, pp. 399-405.

[13] G, Charalampous, C, Hadjiyiannis, Y, Hardalupas, and A, Taylor. "Measurement of continuous liquid jet length in atomizers with optical connectivity, electrical conductivity and high-speed photography techniques". In: .. on Liquid ... September. 2010, pp. 1-10.

[14] Kastengren, Alan and Powell, Christopher F. "Synchrotron X-ray techniques for fluid dynamics". In: Experiments in Fluids 55.3 (2014). DOI: $10.1007 / \mathrm{s} 00348-014-1686-8$. 
[15] Gorokhovski, Mikhael and Herrmann, Marcus. "Modeling Primary Atomization". In: Annual Review of Fluid Mechanics 40.1 (2008), pp. 343366. DOI: 10.1146/annurev.fluid.40.111406.102200.

[16] Pitsch, H. and Desjardins, Olivier. "Detailed Numerical Investigation of Turbulent Atomization of Liquid Jets". In: Atomization and Sprays 20.4 (2010), pp. 311-336. DOI: 10.1615/atomizspr.v20.i4.40.

[17] Marmottant, Philippe. "Atomisation d'un jet liquide par un courant gazeux". PhD thesis. 2001.

[18] Marmottant, P. H. and Villermaux, E. "On spray formation". In: Journal of Fluid Mechanics 498.498 (2004), pp. 73-111. DOI: 10.1017 / S0022112003006529.

[19] Marmottant, Philippe and Villermaux, Emmanuel. "Fragmentaion of stretched liquid ligaments". In: Physics of Fluids 16.8 (2004), pp. 27322741. DOI: $10.1063 / 1.1756030$.

[20] ECN. ECN Webpage.

[21] Payri, Raul, Gimeno, Jaime, Bracho, Gabriela, and Bautista, Abian. "Virtual injection modelling for ECN Spray G". In: Iclass 2018. Chicago, 2018, pp. 1-8.

[22] Berrocal, Edouard, Sedarsky, David L., Paciaroni, Megan E., Meglinski, Igor V., and Linne, Mark A. "Laser light scattering in turbid media Part I: Experimental and simulated results for the spatial intensity distribution". In: Optics Express 15.17 (2007), p. 10649. DOI: 10.1364/ oe.15.010649.

[23] Linne, Mark, Sedarsky, David, Meyer, Terrence, Gord, James, and Carter, Campbell. "Ballistic imaging in the near-field of an effervescent spray". In: Experiments in Fluids 49.4 (2010), pp. 911-923. DOI: $10.1007 / \mathrm{s} 00348-010-0883-3$.

[24] Linne, M. et al. "Correlation of internal flow and spray breakup for a fuel injector used in ship engines". In: 8th US National Combustion Meeting 20132 (2013), pp. 1-8.

[25] Duran, S. P., Porter, J. M., and Parker, T. E. "Ballistic Imaging of Sprays at Diesel Relevant Conditions". In: 12th International Conference on Liquid Atomization and Spray Systems. 2012, pp. 1-5. 
[26] Berrocal, Edouard, Kristensson, Elias, Richter, Mattias, Linne, Mark, and Alden, Marcus. "Multiple Scattering Suppression in Planar Laser Imaging of Dense Sprays By Means of Structured Illumination". In: Atomization and Sprays 20.2 (2010), pp. 133-139. DOI: 10.1615 / atomizspr.v20.i2.30.

[27] Kristensson, E., Berrocal, E., Richter, M., Pettersson, S.-G., and Aldén, M. "High-speed structured planar laser illumination for contrast improvement of two-phase flow images". In: Optics Letters 33.23 (2008), p. 2752. DOI: $10.1364 /$ ol .33.002752.

[28] Berrocal, E., Kristensson, E., Hottenbach, P., Aldén, M., and Grünefeld, G. "Quantitative imaging of a non-combusting diesel spray using structured laser illumination planar imaging". In: Applied Physics B: Lasers and Optics 109.4 (2012), pp. 683-694. DOI: 10.1007/s00340012-5237-9.

[29] Kaiser, Max, Heilig, Ansgar, and Dinkelacker, Friedrich. "Application of the Optical Connectivity Method to a Real Size Heavy Duty CIDIInjector ( Application of the Optical Connectivity Method )". In: $C O$ MODIA 2012. Comodia. 2012, pp. 506-511.

[30] Charalampous, G., Hardalupas, Y., and K. P. Taylor, A. M. "Novel Technique for Measurements of Continuous Liquid Jet Core in an Atomizer". In: AIAA Journal 47.11 (2009), pp. 2605-2615. DOI: 10.2514/ 1.40038 .

[31] Crua, Cyril, Heikal, Morgan R., and Gold, Martin R. "Microscopic imaging of the initial stage of diesel spray formation". In: Fuel 157 (2015), pp. 140-150. DOI: 10.1016/j.fuel.2015.04.041.

[32] Pickett, Lyle M., Manin, Julien, Kastengren, Alan L, and Powell, Christopher F. "Comparison of Near-Field Structure and Growth of a Diesel Spray Using Light-Based Optical Microscopy and X-Ray Radiography". In: SAE Technical Paper 2014-01-1412 7.2 (2014), pp. 10441053. DOI: $10.4271 / 2014-01-1412$.

[33] Wang, Yujie et al. "Ultrafast X-ray study of dense-liquid-jet flow dynamics using structure-tracking velocimetry". In: Nature Physics 4.4 (2008), pp. 305-309. DOI: 10.1038/nphys840.

[34] Bravin, Alberto. "Exploiting the x-ray refraction contrast with an analyser: The state of the art". In: Journal of Physics D: Applied Physics 36.10 A (2003). DOI: 10.1088/0022-3727/36/10A/306. 
[35] Gureyev, T. E. et al. "Refracting Röntgen's rays: Propagation-based x-ray phase contrast for biomedical imaging". In: Journal of Applied Physics 105.10 (2009). DOI: 10.1063/1.3115402.

[36] Moon, S. et al. "Ultrafast X-ray Phase-Contrast Imaging of High-Speed Fuel Sprays from a Two-Hole Diesel Nozzle". In: 22nd Annual Conference on Liquid Atomization and Spray Systems (ILASS Americas 2010) May (2010).

[37] Linne, Mark. "Analysis of X-ray phase contrast imaging in atomizing sprays". In: Experiments in Fluids 52.5 (2012), pp. 1201-1218. DOI: 10.1007/s00348-011-1251-7.

[38] Kastengren, Alan et al. "Correlation of Split-Injection Needle Lift and Spray Structure". In: SAE Technical Paper Series 1 (2011). DoI: 10. 4271/2011-01-0383.

[39] Kastengren, A. et al. "Measurements of droplet size in shear-driven atomization using ultra-small angle x-ray scattering". In: International Journal of Multiphase Flow 92 (2017), pp. 131-139. DOI: 10.1016/j . ijmultiphaseflow.2017.03.005.

[40] Ilavsky, Jan and Jemian, Peter R. "Irena: Tool suite for modeling and analysis of small-angle scattering". In: Journal of Applied Crystallography 42.2 (2009), pp. 347-353. DOI: 10.1107/S0021889809002222.

[41] Battistoni, Michele et al. "Experimental and Computational Investigation of Subcritical Near-Nozzle Spray Structure and Primary Atomization in the Engine Combustion Network Spray D". In: SAE Technical Paper 2018-01-0277 (2018), pp. 1-15. DOI: 10.4271/2018-01-0277.

[42] Vaudor, G., Ménard, T., Aniszewski, W., Doring, M., and Berlemont, A. "A consistent mass and momentum flux computation method for two phase flows. Application to atomization process". In: Computers and Fluids 152 (2017), pp. 204-216. DOI: 10 . $1016 /$ j . compfluid. 2017.04 .023$.

[43] Shinjo, J. and Umemura, A. "Detailed simulation of primary atomization mechanisms in Diesel jet sprays (isolated identification of liquid jet tip effects)". In: Proceedings of the Combustion Institute 33.2 (2011), pp. 2089-2097. DOI: 10.1016/j.proci.2010.07.006.

[44] Elghobashi, Said. "Direct Numerical Simulation of Turbulent Flows Laden with Droplets or Bubbles". In: Annual Review of Fluid Mechanics 51.1 (2018), pp. 217-244. DOI: 10.1146/annurev-fluid-010518040401. 
[45] Balachandar, S. and Eaton, John K. "Turbulent Dispersed Multiphase Flow". In: Annual Review of Fluid Mechanics 42.1 (2010), pp. 111-133. DOI: 10.1146/annurev.fluid.010908.165243.

[46] Prakash, Vivek N. et al. "Energy spectra in turbulent bubbly flows". In: Journal of Fluid Mechanics 791 (2016), pp. 174-190. DOI: 10.1017/ jfm.2016.49.

[47] Ménard, T, Tanguy, S, and Berlemont, A. "Coupling level set/VOF/ghost fluid methods: Validation and application to 3D simulation of the primary break-up of a liquid jet". In: International Journal of Multiphase Flow 33.5 (2007), pp. 510-524. DOI: 10.1016/j.ijmultiphaseflow.2006.11.001.

[48] Klein, M., Sadiki, A., and Janicka, J. "A digital filter based generation of inflow data for spatially developing direct numerical or large eddy simulations". In: Journal of Computational Physics 186.2 (2003), pp. 652-665. DOI: 10.1016/S0021-9991(03)00090-1.

[49] Lebas, R., Menard, T., Beau, P.A., Berlemont, A., and Demoulin, François-Xavier. "Numerical simulation of primary break-up and atomization: DNS and modelling study." In: International Journal of Multiphase Flow 35.3 (2009), pp. 247-260. DOI: 10 . 1016 / j . ijmultiphaseflow.2008.11.005.

[50] Duret, B., Reveillon, J., Menard, T., and Demoulin, F. X. "Improving primary atomization modeling through DNS of two-phase flows". In: International Journal of Multiphase Flow 55 (2013), pp. 130-137. DOI: 10.1016/j.ijmultiphaseflow.2013.05.004.

[51] Canu, Romain et al. "Where does the droplet size distribution come from?" In: International Journal of Multiphase Flow 107.January 2019 (2018), pp. 230-245. DOI: 10.1016/j . ijmultiphaseflow.2018.06. 010.

[52] Shinjo, J. and Umemura, Akira. "Simulation of liquid jet primary breakup: Dynamics of ligament and droplet formation". In: International Journal of Multiphase Flow 36.7 (2010), pp. 513-532. DOI: 10. 1016/j.ijmultiphaseflow.2010.03.008.

[53] Shinjo, J. and Umemura, A. "Surface instability and primary atomization characteristics of straight liquid jet sprays". In: International Journal of Multiphase Flow 37.10 (2011), pp. 1294-1304. DOI: 10.1016/j . ijmultiphaseflow.2011.08.002. 
[54] Umemura, Akira. "Turbulent atomization subgrid model for two-phase flow large eddy simulation (theoretical development)". In: Combustion and Flame 165 (2016), pp. 154-176. DOI: 10.1016/j.combustflame. 2015.11 .025 .

[55] Umemura, Akira and Shinjo, Junji. "Detailed SGS atomization model and its implementation to two-phase flow LES". In: Combustion and Flame 195 (2018), pp. 232-252. DOI: 10.1016/j . combustflame. 2018. 01.026 .

[56] Jarrahbashi, D. and Sirignano, W. A. "Invited Article: Vorticity dynamics for transient high-pressure liquid injection". In: Physics of Fluids 26.10 (2014). DOI: 10.1063/1.4895781.

[57] Zandian, A., Sirignano, W. A., and Hussain, F. "Understanding liquidjet atomization cascades via vortex dynamics". In: Journal of Fluid Mechanics 843 (2018), pp. 293-354. DOI: 10.1017/jfm.2018.113.

[58] Lozano, Antonio, García-Olivares, Antonio, and Dopazo, César. "The instability growth leading to a liquid sheet breakup". In: Physics of Fluids 10.9 (1998), pp. 2188-2197. DOI: 10.1063/1.869740.

[59] Jarrahbashi, D., Sirignano, W. A., Popov, P. P., and Hussain, F. "Early spray development at high gas density: hole, ligament and bridge formations". In: Journal of Fluid Mechanics 792 (2016), pp. 186-231. DOI: 10.1017/jfm.2016.71.

[60] Zandian, A., Sirignano, W. A., and Hussain, F. "Planar liquid jet: Early deformation and atomization cascades". In: Physics of Fluids 29.6 (2017). DOI: 10.1063/1.4986790.

[61] Ling, Y., Fuster, D., Tryggvason, G., and Zaleski, S. "A two-phase mixing layer between parallel gas and liquid streams: Multiphase turbulence statistics and influence of interfacial instability". In: Journal of Fluid Mechanics 859 (2019), pp. 268-307. DOI: 10.1017/jfm. 2018. 825.

[62] Ling, Yue, Fuster, Daniel, Zaleski, Stéphane, and Tryggvason, Grétar. "Spray formation in a quasiplanar gas-liquid mixing layer at moderate density ratios: A numerical closeup". In: Physical Review Fluids 2.1 (2017), p. 014005. DOI: 10.1103/PhysRevFluids.2.014005.

[63] Ling, Y., Zaleski, S., and Scardovelli, R. "Multiscale simulation of atomization with small droplets represented by a Lagrangian pointparticle model". In: International Journal of Multiphase Flow 76 (2015), pp. 122-143. DOI: 10.1016/j.ijmultiphaseflow. 2015.07. 002. 
[64] Desjardins, Olivier, Moureau, Vincent, and Pitsch, Heinz. "An accurate conservative level set/ghost fluid method for simulating turbulent atomization". In: Journal of Computational Physics 227.18 (2008), pp. 8395-8416. DOI: $10.1016 / j \cdot j c p .2008 .05 .027$.

[65] "On simulating primary atomization using the refined level set grid method". In: Atomization and Sprays 21.4 (2011), pp. 283-301. DOI: 10.1615/AtomizSpr. 2011002760.

[66] Kolmogorov, A. N. "The Local Structure of Turbulence in Incompressible Viscous Fluid for Very Large Reynolds Numbers". In: Proceedings of the Royal Society A: Mathematical, Physical and Engineering Sciences 434.1890 (1991), pp. 9-13. DOI: 10.1098/rspa.1991.0075.

[67] Hinze, J. O. and Shell-laboratory, Royal Dutch. "Fundamentals of the hydrodynamic mechanism of splitting in dispersion processes". In: AIChE Journal 1.3 (1955), pp. 289-295. DOI: 10 . 1002 / aic . 690010303.

[68] Batchelor, G. K., Howells, I. D., and Townsend, A. A. "Small-scale variation of convected quantities like temperature in turbulent fluid: Part 2. The case of large conductivity". In: Journal of Fluid Mechanics 5.1 (1959), pp. 134-139. DOI: 10.1017/S0022112059000106.

[69] "A sub-grid surface dynamics model for sub-filter surface tension induced interface dynamics". In: Computers and Fluids 87.480 (2013), pp. 92-101. DOI: 10.1016/j.compfluid.2013.02.008.

[70] Hasslberger, Josef, Ketterl, Sebastian, Klein, Markus, and Chakraborty, Nilanjan. "Flow topologies in primary atomization of liquid jets: A direct numerical simulation analysis". In: Journal of Fluid Mechanics 859 (2019), pp. 819-838. DOI: 10.1017/jfm.2018.845.

[71] Payri, Raul, Gimeno, Jaime, Marti-Aldaravi, Pedro, and Bracho, Gabriela. "Study of the influence of the inlet boundary conditions in a LES simulation of internal flow in a diesel injector". In: Mathematical and Computer Modelling 57.7-8 (2013), pp. 1709-1715. DOI: 10.1016/j.mcm.2011.11.019.

[72] Payri, R., Tormos, B., Gimeno, J., and Bracho, G. "The potential of Large Eddy Simulation (LES) code for the modeling of flow in diesel injectors". In: Mathematical and Computer Modelling 52.7-8 (2010), pp. 1151-1160. DOI: $10.1016 / \mathrm{j} . \mathrm{mcm} .2010 .02 .033$. 
[73] Keating, Anthony, Piomelli, Ugo, Balaras, Elias, and Kaltenbach, Hans Jakob. "A priori and a posteriori tests of inflow conditions for largeeddy simulation". In: Physics of Fluids 16.12 (2004), pp. 4696-4712. DOI: $10.1063 / 1.1811672$.

[74] Balarac, Guillaume and Métais, Olivier. "The near field of coaxial jets: A numerical study". In: Physics of Fluids 17.6 (2005), pp. 1-14. DOI: 10.1063/1.1900786.

[75] Fellouah, H., Ball, C. G., and Pollard, A. "Reynolds number effects within the development region of a turbulent round free jet". In: International Journal of Heat and Mass Transfer 52.17-18 (2009), pp. 39433954. DOI: 10.1016/j.ijheatmasstransfer.2009.03.029.

[76] Vassallo, P. and Ashgriz, N. "Satellite Formation and Merging in Liquid Jet Breakup". In: Proceedings of the Royal Society A: Mathematical, Physical and Engineering Sciences 433.1888 (2006), pp. 269-286. DOI: 10.1098/rspa.1991.0047.

[77] Gorokhovski, M. A. and Saveliev, V. L. "Analyses of Kolmogorov's model of breakup and its application into Lagrangian computation of liquid sprays under air-blast atomization". In: Physics of Fluids 15.1 (2003), pp. 184-192. DOI: 10.1063/1.1527914.

[78] Kolmogorov, A. "On the log-normal distribution of particles sizes during breakup process". In: Dokl. Akad. Nauk. XXXI.4 (1941), pp. 99101.

[79] Bassenne, Maxime, Moin, Parviz, and Urzay, Javier. "Wavelet multiresolution analysis of particle-laden turbulence". In: Physical Review Fluids 3.8 (2018), p. 084304. DOI: 10.1103/PhysRevFluids.3.084304. 



\section{Chapter 2}

\section{Overview of numerical methods in multiphase flows.}

\subsection{Numerical framework requirements}

The choice of a numerical framework when performing DNS of sprays is a key component in the research design and the drivers are not only dictated by scientific reasons. In fact, the computer architectures on which the simulations will be performed is as important as the scientific design, as it dictates the size of the problem you will be able to solve and, therefore, its physics. Writing an entire high-performance scalable code may require significant time for the development, validation and optimization phases and can easily take several years and the involvement of people with different expertises. This is outside the scope of this thesis, in which a code framework was used and modified in order to study a specific problem. For this reason, a discussion about the numerical constrain required by the study of a turbulent spray dense region will now be addressed.

For what discussed in Chapter 1 most of the applications which can be studied using DNS have typically low Re numbers. Although it is not always the case, this usually corresponds with low Mach number $(M a)$. For $M a<0.3$ the compressibility effects are negligible, hence the flow can be considered incompressible. In sprays, the flow with the highest compressibility is the gas phase, hence the worst case scenario is always computed using the injection velocity and the gas speed of sound. If needed, a slight compressibility can be 
added by using an All-Mach method, which proved to be easy to implement in multiphase flows [1].

The mesh type is also extremely relevant in choosing the numerical framework. As discussed in Chapter 1, even when the turbulence scales are well resolved, the flow surface feature (especially surface curvature) may still be unresolved [2]. Solving both turbulent and multiphase smallest scales may quickly lead to an unmanageable number of cells in the simulation, which may result in an extremely large (and likely unfeasible) simulation. For this reasons, many authors have tried to come up with method that could still solve the problem from a physical stand point while reducing the number of cells. Various successful attempt have been made with cylindrical meshing and, in general, complex geometries [3, 4]. The ill-conditioned Poisson equation in these cases forces the adoption of more sophisticated solvers, such as multigrid type, that may not be always an optimal choice for the code performances. This mesh can be decoupled in the Navier-Stokes grid and the surface refinement grid, as described by "On simulating primary atomization using the refined level set grid method" [4]. This method requires many computational manipulation in order to maintain a proficient parallelization and load balance hence is not always the preferred choice. NGA [5] is an example of code that has the option of using cylindrical meshes, complex geometries and is massively scalable.

Adaptive Mesh Refinement (AMR) techniques have proved to be an extremely effective way to reduce the mesh elements while performing accurate simulations. This type of mesh is extremely well suited to solve complex flows with low Reynolds numbers but some issues may arise with highly turbulent flows. In fact, AMR usually refines due to high values of spatial gradient of vorticity and liquid-gas surface curvature, theoretically covering both the needs imposed by the smallest scales in turbulent and multiphase flows. On the other hand, it is still unclear how AMR causality influences the correct calculations of turbulence and if the usage of larger cells may imply a lowpass filter in the wave propagations. Furthermore, as the number of elements changes continuously in codes with AMR, the scaling up to thousands of cores (needed for performing this study) may be inefficient. While this method may be very well suited for the application discussed in this thesis, these uncertainties discouraged its usage for the turbulence studies performed here. On the other hand it should be noted (and will be addressed towards the hand) that an a-priori knowledge of the turbulent field may help in guiding this type of codes and remove the uncertainties. The most notable examples are Gerris [6] and Basilisk [7] which both uses octree based mesh-refinement [8] with an efficient implementation of the load balance. 
Finally, the most widely used mesh type is the cartesian mesh, where all the cells are cuboid. There are undoubtedly advantages and disadvantages to this approach but for many reasons it gained significant popularity among researchers, as for most of the studies in Chapter 1 ([9-12] among others). In this type of mesh, of course, all the spatial frequencies up to the one resolvable with the cell size are captured and solved while no refinement is needed. Domain are usually prisms therefore the computational load is split evenly among the processes (regular array sub-domain parallelization) hence their strong and weak scalability is often almost perfect. Furthermore, the results are ideally sorted for simple and intuitive post-processing. On the other hand this mesh generates a significant amount of data that need to be handled and selected wisely, often forcing into detailed preliminary study of the case studied in order to preventively set a focus on the needed post-process output. Some notable examples are ARCHER [13] and ParisSimulator [14].

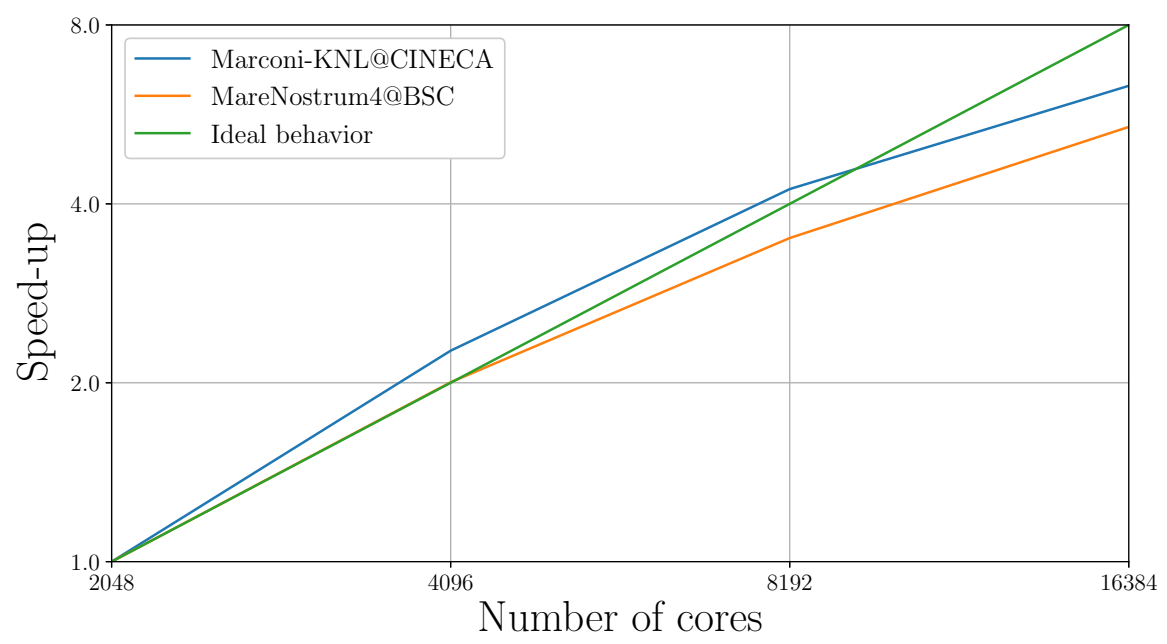

Figure 2.1: ParisSimulator code scalability on Marconi and MareNostrum4 supercomputers.

The numerical framework finally chosen for this thesis is ParisSimulator, for a number of reasons significant in the context of this work. In a first place, its code structure offers a simple environment where programming additional code features, such as the boundary conditions that are needed to perform this study. The code parallelization, due to its simple code-structure, is very efficient and the memory usage is contained. Both these features are of key importance for simulating on massive-parallel petascale systems. An example of the code scalability computed in this thesis for a spray case is reported in 
Figure 2.1. Finally, as already discussed, the exact knowledge of the effects given by a cartesian grid without refinement is highly valuable for the success of this work.

In this section, a description of the main numerical methods available for multiphase flows will be presented. The emphasis will be on the methods in ParisSimulator, although other significant methods (e.g. Level-Set) will be presented. The numerical implementation and discretization are outside of the scope of this thesis, therefore they will be discussed only when necessary (see [15] for a more in depth discussion of the topic). Some very detailed reviews about the topic addressed in the following section have been written by Popinet [16], Scardovelli and Zaleski [17], and Gorokhovski and Herrmann $[18]$.

\subsection{Basic equations for multiphase flows}

Although many real industrial applications experience drastic changes in temperatures, the study of primary atomization is often carried over in isothermal and adiabatic conditions. This assumption removes the need for solving the energy equation and has still a significant validity for most experiments performed at ambient temperature. Summing this hypothesis with the incompressible flow one, the continuity equations for multiphase flows can be written as :

$$
\begin{gathered}
\nabla \cdot \mathbf{u}=0 \\
\rho\left(\partial_{t} \mathbf{u}+\mathbf{u} \cdot \nabla \mathbf{u}\right)=-\nabla p+\mu \nabla \cdot\left(\partial_{i} u_{j}+\partial_{j} u_{i}\right)+\sigma \kappa \delta_{s} \mathbf{n}
\end{gathered}
$$

In the above equations, $\mathbf{u}$ represents the velocity field, which due to the incompressibility hypothesis results to be divergence free, as stated in Equation (2.1a). The pressure is represented by $p$, while the properties $\rho$ and $\mu$ are respectively the fluid density and dynamic viscosity in the evaluated point in space.

A special treatment needs to be devoted to the the third and last term on the right hand side of Equation (2.1b), which represents the contribution provided by the liquid interface. This term is non-zero when the Dirac function $\delta$ is one, which occurs only on the surface. In case of sharp interface, this term would require an infinitely refined mesh size in order to be activated in the equation, but that is obviously not the case in numerical simulation, hence 
a special treatment will be necessary to detect where the surface lies (within which cell). The surface tension $\sigma$ contribution is therefore evaluated using the liquid surface curvature $\kappa$ and the surface normal $\mathbf{n}$.

Regardless of the method used to address the multiphase nature of the flow (which will be discussed later) an estimation of the properties is required. This is commonly achieved by using an Heaviside function, which describes an abrupt and sharp change between the two fluids. Using an Heaviside function is an inherently accurate method for describing the sharp interface between fluids, regardless of how the surface advection is treated. The calculation of the fluid properties can therefore be described as:

$$
\begin{aligned}
& \rho(\mathbf{x})=\rho_{l} H(\mathbf{x})+\rho_{g}(1-H(\mathbf{x})) \\
& \mu(\mathbf{x})=\mu_{l} H(\mathbf{x})+\mu_{g}(1-H(\mathbf{x}))
\end{aligned}
$$

where $H(\mathbf{x})$ is the Heaviside function, which is obviously a function of the spatial position. This linear interpolation of properties becomes physically exactly true when the interface is sharp.

A more detailed description of the equations can be found in [15], where also the whole energy equation discussion and the compressible flow case are addressed.

\subsection{Solution of the Navier-Stokes equation}

The numerical solution of the Navier-Stokes equation is a topic extensively addressed in literature and many methods can be found in textbooks. In this case, the explanation is focused on the methods used in finite volume multiphase flows, with a focus on the methods used in ParisSimulator. The whole discussion about this method (from which this description is derived), can be found in [15]. First, we rewrote in Equation (2.1b) in a format more suitable for migrate into the discrete form:

$$
\rho \partial_{t} \mathbf{u}+\rho \mathcal{A}=-\nabla p+\mathcal{D}+\mathcal{F}
$$

where $\mathcal{A}=\mathbf{u} \cdot \nabla \mathbf{u}$ is the advection term, $\mathcal{D}=\mu \nabla \cdot\left(\partial_{i} u_{j}+\partial_{j} u_{i}\right)$ is the diffusion term and $\mathcal{F}$ represents the other source terms, which in our case is simply the surface tension. The discrete form of Equation (2.3) depends on 
which order is picked for discretization schemes. Usually, the best practice is using higher order methods (at least second), but in order to keep the discussion simple and focus on the methods, a simple first order will be used in the following. It is worth noting that in the simulation performed in this thesis, both time and space integration are second-order accurate.

In the following sections, special attention will be devoted to the timesolution of Equation (2.3). The spatial integration will be qualitatively addressed, as there are a significant number of methods for addressing these flows and their discussion will not be significant for the results presented in this thesis.

\subsubsection{The Chorin projection method}

One of the most diffused methods for performing the time integration is the Chorin projection method [19], which is extremely common in DNS of multiphase flow. The name derives by the method's nature, for which 2 steps are used.First, a "first attempt" velocity is computed from Equation (2.3) by imposing a zero pressure gradient. This velocity is usually not divergence free, therefore the second step uses the pressure field to correct the first attempt velocity. This operation is formally called a projection into the divergence-free velocity field, hence the method's name. The scheme is completely explicit and can be easily extended to second order, although here is presented in first order for reasons that will soon be evident.

Solving Equation (2.3) using a first order accurate solution in time, the time derivatives can be written as:

$$
\partial_{t} \mathbf{u}=\frac{\mathbf{u}^{n+1}-\mathbf{u}^{n}}{\Delta t}=\frac{\left(\mathbf{u}^{n+1}-\mathbf{u}^{*}\right)+\left(\mathbf{u}^{*}-\mathbf{u}^{n}\right)}{\Delta t}
$$

where $n$ and $n+1$ are respectively the current and the next time-steps, $\Delta t$ is the time-step used and $u^{*}$ is the temporal velocity used for the projection. In order to find $\mathbf{u}^{n+1}$, the last formulation of Equation (2.4) is solved in different steps. First the second parenthesis is solved for $\mathbf{u}^{*}$ as:

$$
\frac{\mathbf{u}^{*}-\mathbf{u}^{n}}{\Delta t}=-\mathcal{A}^{n}+\frac{\mathcal{D}^{n}+\mathcal{F}^{n}}{\rho^{n}}
$$

where the pressure contribution is missing. The pressure term can be found by imposing that the velocity $\mathbf{u}^{*}$ is divergence free in Equation (2.3) 
and solving just for the pressure terms, which lead to:

$$
\frac{\nabla \cdot \mathbf{u}^{*}}{\Delta t}=\nabla \cdot\left(\frac{\nabla p}{\rho^{n}}\right)
$$

Finally, the projection step is performed as :

$$
\frac{\mathbf{u}^{n+1}-\mathbf{u}^{*}}{\Delta t}=\frac{\nabla p}{\rho^{n}}
$$

The extension of this method to second order, especially for coding purposes is extremely easy and basically can be summarized into a projectioncorrection algorithm. In simple terms, the algorithm proposed for the first order can be repeated twice, while the resulting velocity is:

$$
\mathbf{u}^{n+1}=\left(\mathbf{u}^{n}+\mathbf{u}^{n+2}\right) / 2
$$

Please note that, in this case, the exponent $n+2$ has only a mere programming meaning, not a temporal one.

The projection method is vastly used in literature and it can be extended also to variable material properties [20]. Alternatives to this methods are the well-known PISO and SIMPLE methods, that will not be discussed as they are not of direct interest in this thesis and, often, they are computationally more demanding than the projection method.

\subsubsection{Spatial integration}

The two main storage system for variables depends on whether a collocated or staggered grids are used. The former is the most intuitive one, where both pressure and velocity are stored at cell centers, while the latest uses pressure and material properties stored at the cell center and velocity stored at the cells faces. In the latest, material properties are located at cell center, while velocity is located at cell faces. Extensive discussions have been presented in literature on whether one is better than the other [15], but experience shows that staggered grid are more suitable for incompressible flows. In fact, staggered grid have been reportedly being:

- More accurate than collocated grids: the intuitive result of having variables stored both on cell centers and edges is that the solution is inherently performed on some type of finer grid (although not as accurate as collocated with half the grid-size). 
- Conservative methods are simpler to produce.

- Better coupling between the variables: staggered grid produce less numerical fluctuations and still connects all the cells on a numerical stencil together.

The whole numerical discretization of Equation (2.3) is tedious and do not provide a significant scientific insight on the thesis topic. On the other hand, an overall comprehension of the schemes available to numerically integrate the momentum equation is needed in order to understand why some methodologies have been preferred in the DNS works presented in Chapter 1.

\section{Advection term}

The advection term $\mathcal{A}$ can be computed using various methods and its derivation in multiphase flows has no differences with the single phase one, being involved purely kinematic variables. The main methods used for the advection term discretization are:

- QUICK: acronym for Quadratic Upstream Interpolation for Convective Kinetics. This method, developed by Leonard [21], is a upstream quadratic interpolation (hence three points are used) for cell-faces values. The method is third-order accurate and provide a significant improvement in respect to upwind first-order methods, reducing significantly numerical oscillation. This method is extremely diffused in a number of CFD codes.

- ENO: Essentially Non-Oscillating method by Shu and Osher [22]. It relies on the extrapolation of the cell face values into the cell center, using a minim-slope criteria. A successive implementation in its weighted version (WENO) from Liu et al. [23] is the most popular version of the model, where the gradients are weighted for finding the cell-center extrapolated value. As QUICK, it is well known for its robustness but it is also often used in higher order versions (although its basic version is second-order).

- Bell-Colella-Glaz: it is basically a variation of the ENO method, developed by Bell et al. [24]. This method is mentioned as widely used in multiphase flow DNS (e.g. [6, 7]). 


\subsubsection{Viscous term}

The continous integral of the viscous term, $\mathcal{D}$, is defined as:

$$
\mathcal{D}=\frac{1}{V} \oint_{S} \mu\left(\partial_{i} u_{j}+\partial_{j} u_{i}\right) d s
$$

where $S$ is the surface over which the fluxes is calculated. From what discussed in Section 2.2, it is obvious that the main concern for the numerical integration of this equation is represented by the behavior at the interface. The presence of $\mu$ can be addressed by Equation (2.2b), although this equation proves to be a correct approximation only if $\mu_{l} \approx \mu_{g}$. In this case the discontinuity is less pronounced and a step function is a sufficient approximation of the physical behavior. On the other hand, as extensively discussed in literature (e.g. [15]), a significant difference in the viscosity lead to a discontinuous derivative across the surface in the discretized form of Equation (2.9). For reasons that will be discussed in Section 2.4, this method can be directly used with the VOF method, while Level-Set and Front Tracking will require further treatments in order to solve this issue. On the other hand, using an harmonic mean leads towards a more "noisy" method. In fact, it is known [15], that arithmetic mean over-predict the viscosity, which reduce the amount of short wavelength noise frequency that perturb the liquid surface. A second order accurate method for solving this issue using a second order accurate approach has not yet been published [15].

\subsubsection{Methods for solving the pressure equation}

Equation (2.6) is a Poisson equation that can be solved by using different methods. For what has been discussed until now, after performing the choice of which spatial numerical scheme to adopt (Sections 2.3.2 and 2.3.3) and if surface can be located (using one of the methods presented in Section 2.4), we have all the elements to solve Equation (2.6). If we determine all the terms $\mathcal{A}, \mathcal{D}$ and $\mathcal{F}$ to compute $\mathbf{u}^{*}$, the discrete form of Equation (2.6) reads (for example in $1 \mathrm{D}$ staggered grids):

$$
p_{i}^{\iota+1}=\frac{\frac{1}{\Delta x^{2}}\left(\frac{p_{i+1}^{\iota}}{\rho_{i+1}^{\iota}+\rho_{i}^{\iota}}+\frac{p_{i-1}^{\iota}}{\rho_{i-1}^{\iota}+\rho_{i}^{\iota}}\right)+\frac{u_{i+1 / 2}^{*}-u_{i-1 / 2}^{*}}{2 \Delta t \Delta x}}{\frac{1}{\Delta x^{2}}\left(\frac{1}{\rho_{i+1}^{\iota}+\rho_{i}^{\iota}}+\frac{1}{\rho_{i-1}^{\iota}+\rho_{i}^{\iota}}\right)}
$$

which can be easily extended in three dimensions or directly found on books $[15,25]$. Here, $\iota$ is the iteration step and the whole equation can be 
solved with an iterative method among the ones listed below. A significant number of more sophisticated methods, like the Bi Conjugate Gradient Stabilized (BiCGSTAB ) from Vorst [26], can be used for very high density ratios, which are far beyond the ones used in sprays and canonical multiphase flows.

\section{Successive Over Relaxation (SOR)}

This methods allows a smoother convergence by accounting for the value in the previous timestep in Equation (2.10) and using a over-relaxation parameter, usually called $\varpi$ :

$$
p_{i}^{\iota+1}=\varpi \frac{\frac{1}{\Delta x^{2}}\left(\frac{p_{i+1}^{\iota}}{\rho_{i+1}^{\iota}+\rho_{i}^{\iota}}+\frac{p_{i-1}^{\iota}}{\rho_{i-1}^{\iota}+\rho_{i}^{\iota}}\right)+\frac{u_{i+1 / 2}^{*}-u_{i-1 / 2}^{*}}{2 \Delta t \Delta x}}{\frac{1}{\Delta x^{2}}\left(\frac{1}{\rho_{i+1}^{\iota}+\rho_{i}^{\iota}}+\frac{1}{\rho_{i-1}^{\iota}+\rho_{i}^{\iota}}\right)}+(1-\varpi) p_{i}^{\iota}
$$

the value of $\varpi$ depends on many aspect of the numerical study performed and is usually picked as high as possible to improve the convergence. The two main iteration methods to solve Equation (2.11) are the Jacobi and the Gauss-Siedel methods. In the Jacobi method, at each iteration the solution is computed using only the information of the iteration before. From a computational standpoint, this requires storing two variable fields, for $p^{\iota+1}$ and $p^{\iota}$, but the independence from the current iteration results makes it perfect for modern architectures (e.g. Graphic Processing Unit, GPU). Despite this small advantage, the convergence speed for this method is quite slow and the GaussSiedel method is often preferred. This method improves the convergence speed by "propagating" the solution. In other words, in Equation (2.11), $p_{i-1}^{\iota+1}$ is used instead of $p_{i-1}^{\iota}$ (assuming that the solution sequence goes from 0 to $N_{i}$, the total number of elements in the $i-t h$ direction). This method is also more memory-efficient, as only one variable for pressure is stored.

\section{Multigrid methods}

The SOR methods, although always convergent for appropriate values of $\varpi$, are very slow in reaching convergence especially for high density ratio flows, which is a common case for multiphase flows. One of the main reason is given by the fact that for fine grids, the maximum time step that can be used is dictated by the grid size $[15,25]$. Moreover, most of the computational time is used to compute solution components that takes a significant amount of iterations in order to propagate through the domain numerically. On the other hand, small wavelength components are quickly solved. 
This consideration has actually lead to the first formulations of multigrid solvers from Briggs et al. [27]. Simply described, the finer grid is first used to iterate and find a first attempt solution. Once the convergence rate decreases (hence higher solution frequencies have been solved) the solution is passed into a larger grid, which is then used to find larger components of the solution. This method progresses until the very coarse grids (the largest possible) is reached and the solution frequencies are added to provide the final result.

A detailed explanation of the methods is outside the scope of this thesis, but it is worth mention that the method can be programmed using both quite simple [25] and complex and sophisticated parallelizable methods [27].

\subsection{Interface treatment}

While most of the numerical methods described above are quite well-known in CFD (regardless of the studied case), the treatment of the interface and its corresponding surface forces is unique of gas-liquid multiphase flows. There have been a very significant literature on the topic, as the evolution of the computational methods used to describe this flows have just developed mainly in the last 30 years. As the method used in this thesis is the VOF, most description effort will be devoted in describing this method and its numerical consequences, while other techniques will only be addressed qualitatively with specific references that will allow readers to gather more information on the matter.

\subsubsection{Defining a surface and its advection}

There are various ways to define the liquid surface. In very simple cases, where no topological changes are present (such as detached droplets), the way to define a continuous surface is by defining it as a function of space, such as $S(\mathbf{x})$. If we now take all the points lying on the surface, such as $\mathbf{x}_{s}$, their motion in time can be wrote as:

$$
\frac{d \mathbf{x}_{s}}{d t}=\mathbf{u}\left(\mathbf{x}_{s}, t\right)
$$

Equation (2.12), seen over a a finite number of points, leads directly into the first class of methods, which are intrinsically Lagrangian methods. These methods named interphase-tracking, among which the Front-Tracking (FT) method will be discussed, aim to follow the surface in a Eulerian mesh by defining Lagrangian points on the surface and describing their motion. 
If, on the other hand, one just wants to use a single grid for the problem solution, the Heaviside function comes in handy and the equation describing its motion is :

$$
\partial_{t} H(\mathbf{x})+\mathbf{u} \nabla H(u)=0
$$

This formulation is entirely Eulerian and the methods that are used to solve this equation are called interface-capturing methods, because contrarily to the interphase-tracking methods, here the position of the surface is unknown and needs to be determined. VOF and LS methods belongs to the interfacecapturing category.

\section{Front-Tracking method}

This method was first developed by Unverdi and Tryggvason [28] and successively expanded by Tryggvason et al. [29]. It describes the liquid surface by an arbitrary number of points on its surface and solves the motion of each one using Equation (2.12).

FT has a number of clear advantages. For instance, the solution of Equation (2.12) is relatively straightforward and the determination of surface curvature and surface forces is less complex than in other methods, as the surface position is known. On the other hand, this method has some clear drawbacks. In a first place, the method is not able to handle topological events, such as ligaments breakup, automatically but instead requires a manual intervention. Such task automation is not a computationally easy task when the priority is to maintain an efficient implementation of the algorithm. Another downside, although relatively less complex to solve, is the definition of a Heaviside function so that the liquid properties can be computed using Equation (2.2). Furthermore when there are significant velocity gradients and consequently surface deformations this method needs to undergo a re-meshing of the surface, in order to ensure a reliable description of it by its markers.

It is clear that this method is definitely unsuited for sprays DNS, but its value for more theoretical studies is undeniable. On the other hand, this method is perfectly suited for addressing fundamental studies thanks to its accurate description of the liquid surface. Instability propagation studies and bubbly flows (e.g. [30,31]) are a clear examples of such flows.

\section{Level-Set Method}

LS is an undeniably popular method for multiphase flows and especially for sprays. The reason lies mostly in its simplicity, but few important features 
of this method must be accounted for. The main idea is to define a function that is 0 at the surface, 1 in a region characterized by one fluid and -1 in the region characterized by the other. This smooth function can be called as $F$. As the surface moves with the fluid, it is advected according to Equation (2.13), where $H$ is replaced by $F$.

If the term $\mathcal{F}$ in Equation (2.3) is equal to the surface tension,

$$
\mathcal{F}=\sigma \kappa \delta_{s} \mathbf{n}
$$

the definition of $F$ provided makes the computation of the surface normal trivial:

$$
\mathbf{n}=-\frac{\nabla F}{|\nabla F|}
$$

Equation (2.15) means that no further methods are required in order to reconstruct the surface (like in VOF, as it will be discussed later). Furthermore, some of the advection methods presented in Section 2.3.2 can be used for the flux in $u \nabla F$ thanks to the smoothness of the function. Also, the properties calculation in Equation (2.2) need a Heaviside function, which can be constructed as:

$$
f(x)= \begin{cases}0, & \text { if } F<-\alpha_{F} \Delta x \\ 0.5\left(1+\left(F / \alpha_{F} \Delta x\right)+\sin \left(\pi F / \alpha_{F} \Delta x\right) / \pi\right), & \text { if }|F| \leq \alpha_{F} \Delta x . \\ 1, & \text { if } F>\alpha_{F} \Delta x .\end{cases}
$$

where $\alpha_{F}$ is the thickness of the surface per each side and can be adjusted as wished.

This method is extremely appealing due to the fact that, in principle, no additional methods are required to solve it and it just relies on normal partial differential equations, without the need to reconstruct the surface. Nevertheless, this method is not mass-conserving, which is the major drawback and has forced great sophistications of this method, such as its hybridization with the VOF or the "ghost-fluid" method. This increment in complexity is often decreasing the likelihood of this method in favor of VOF. 


\section{Volume of Fluid}

As this method will be adopted in this thesis, more details will be provided. No special description for momentum conserving methods will be provided as the density ratio used in this work is well below the normal values adopted.

In this method, the color-function $C$ is used instead of the Heaviside function $H$ in Equations (2.2) and (2.13); because of it, the color function $C$ needs to variate between 0 and 1 , which are the extreme values that correspond to the first and the second fluid studied. It is obvious that this definition fits perfectly the needs of Equation (2.2) and no further treatments of $C$ are needed.
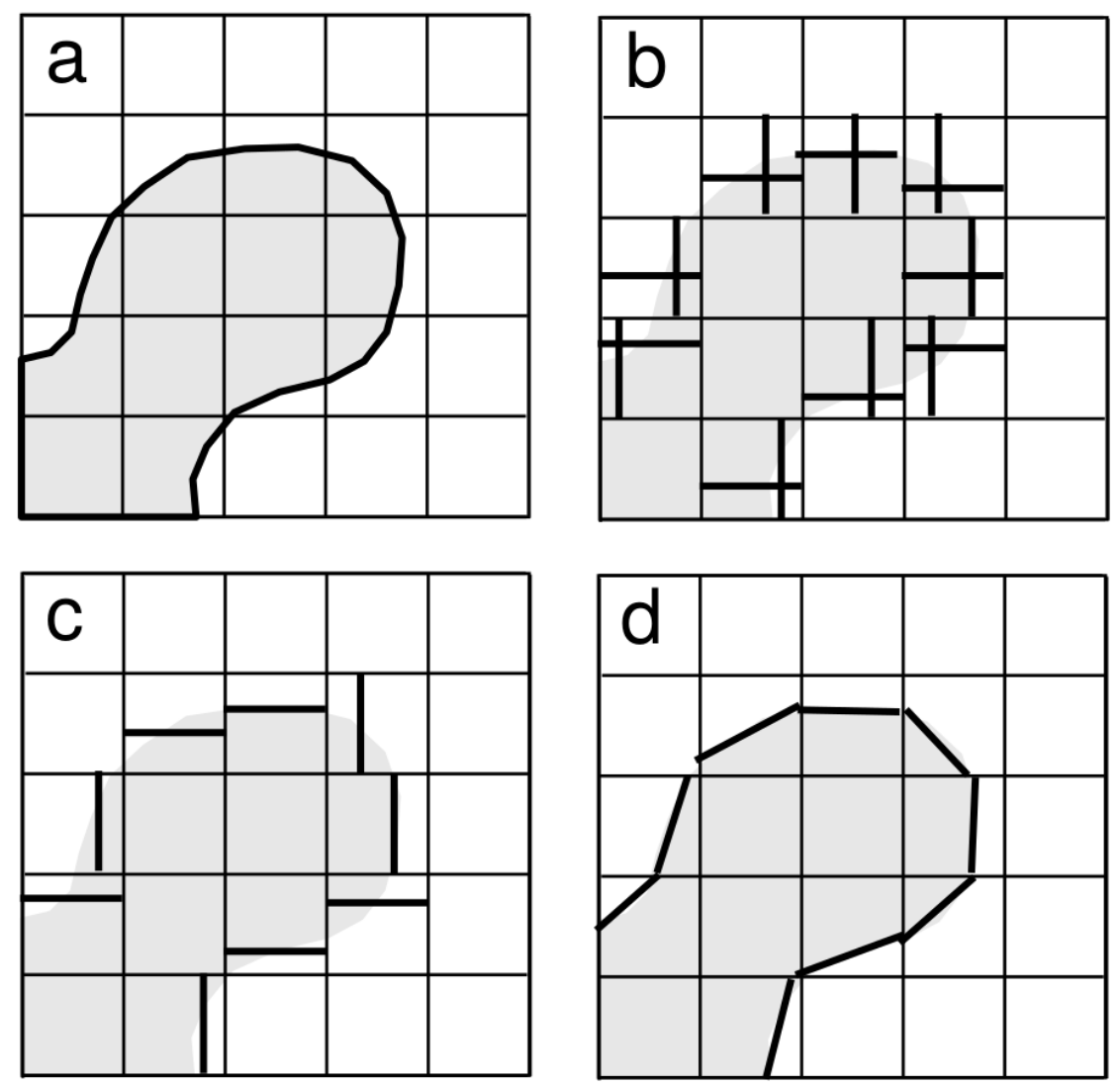

Figure 2.2: Different method for surface reconstruction in VOF (from [15]). $\boldsymbol{a})$ is the original interface, $\boldsymbol{b}$ ) is the SLIC reconstruction, $\boldsymbol{c}$ ) is the reconstruction method proposed by Hirt and Nichols [32] and d) is the PLIC reconstruction. 
The main issue of this method comes in the solution of the advection part of equation, where the flux derivative is performed (second term in Equation (2.13)). While its calculation in one-dimensional problems is trivial [15], its calculation in two and three dimensions needs special treatments. In VOF methods, the field $0<C<1$ means that a cell is partially occupied by liquid. In one-dimension, this means that the surface has to be normal to the direction of propagation of the liquid and its location will be at $C \Delta x$ from the cell face from which the liquid is propagating. In two and three dimensions problems the position of the liquid surface is given by the value of the color function $C$. This problem is well represented in Figure 2.2, taken from the work of Tryggvason et al. [15], adapted from the first explanation of the issue provided by Sussman [33] (please, refer to the caption for the sub figure description). The first attempt in solving this problem was provided by the Simple Line Interface Calculation (SLIC) proposed by Noh and Woodward [34] in 1976. This method was the first to consider the value of the neighbor cells to determine, in each direction, which was the verse of the liquid propagation. Once it was determined, a straight line (normal to the propagation direction considered) was drawn and advected using time-splitting (sequential advection). Once the new position of the surface was found, Equation (2.13) can be solved. A similar approach was undertake by Hirt and Nichols [35] where the surface was approximated by just one segment (or plane). The orientation of the segment had to be parallel to one of the cartesian axes and was decided by computing the normal of the surface in that cell. In order to do so the neighbor cells were once again used as did by Noh and Woodward [34]. The normal was then projected onto the axis in order to find which one was the prominent. Although this first two methods have been popular for a number of years, it has been proved that they provide similar results and significant "artificial" breakup.

A significant improvement for the calculation of the surface advection was provided by the Piecewise Linear Interface Calculation (PLIC) algorithms developed by Youngs [36] and then rediscussed by Sussman [33], "Piecewise Linear Interface Calculation" [37], and Scardovelli and Zaleski [38] for various implementations. Similarly to the SLIC method, PLIC found the surface normal in each cell by using the neighbor values. The surface is then advected in whatever direction its normal dictates, therefore, in this case, finding a good approximation for $\mathbf{n}$ is necessary, as the method accuracy can change significantly. In two and three dimensions this need for accuracy leads to complex geometrical algorithms, on which many authors have different takes and that are discussed in $[15,17]$. 


\subsubsection{Treating the surface tension terms}

Until here, we gained notion on methods available to solve the advection term $\mathcal{A}$, the viscous term $\mathcal{D}$ and how to advect the surface (Equations (2.12) and (2.13)). Using the Chorin projection method ensures a divergence free velocity field, hence also Equation (2.1a) is satisfied. The one term that still need to be addressed is the source force term $\mathcal{F}$, that in this presence work will be reduced to just Equation (2.14) as no buoyancy is accounted for.

The main issues in solving Equation (2.14) are the estimation of the surface normal $\mathbf{n}$ and curvature $\kappa$. Both depend on a correct geometrical description of the liquid surface and on the method used for tracking/capturing it. A significant amount of methods have been developed in literature for achieving a correct estimation of Equation (2.14) while maintaining the computing algorithm as simple as possible, so that its implementation can be computationally efficient. The main methods will be discussed in the following sections, while for a complete review on the methods and their implementation details an indepth review of literature can be found in Tryggvason et al. [15] and Popinet $[16]$.

\section{Continuous Surface Force}

Formulated in 1992 by Brackbill et al. [39], this method is one of the simplest to use and implement and has almost immediate application in both the VOF (being $C$ a direct numerical form of the Heaviside function) and the LS (as $F$ can be described as a Heaviside function by means of Equation (2.16) ). It will be briefly described for the VOF method in the following.

As $C$ is the numerical approximation of $H$, the surface normal is simply computed as

$$
\delta_{s} \mathbf{n}=-\nabla C,
$$

leading to the following equation for the surface tension forces:

$$
\mathcal{F}=-\sigma \kappa \nabla C .
$$

The surface curvature is still required. The usual way to proceed in this method comes from a simple geometrical observation (e.g. see the work of Sussman [33]) for which

$$
\kappa=\nabla \cdot \mathbf{n}
$$


This is the way in which is usually implemented for LS methods. In VOF it is a usual practice to smooth the field $C$ before applying Equation (2.19), which is a class of methods on their own called smoothed CSF and were part of the initial formulation by Brackbill et al. [39]. This method has the advantage of a significant simplicity but it is also subject to notable noise and spurious current propagations. A variant, which is exactly momentum conserving, is the Continuous Surface Stress [15]

\section{Ghost Fluid Method}

Another interesting method for computing the surface tension forces (or, better said, not to) is the Ghost Fluid Method (GSM) by Fedkiw et al. [40]. This method is in fact removing the surface tension force term, while creating a corresponding jump-conditions for pressure at the interface. The second-order discretization of the pressure gradient in one dimension (evaluated at the cell face) is computed as:

$$
\nabla p_{i-1 / 2}=\left(p_{i}-p_{i-1}\right) / \Delta x .
$$

The conditional value of the pressure $p_{i}$ introduced by the GFM is actually modified if the interface is located between $x_{i-1} \div x_{i}$, so that its new value is $p_{i}^{*}=p_{i} \pm \sigma \kappa$.

While this method was originally developed for Level-Set, it is also widely used for VOF. Obviously, this method is not smooth and provide a sharp Heaviside function [16].

\subsubsection{Height Function method}

Among the methods presented until this point, the Height Function (HF) is the most complex from a geometrical standpoint and also the most popular in multiphase DNS codes. In this case it will be presented for VOF algorithm.

Lets call the HF $h$ in a two dimensional case, sketched in Figure 2.3 where each direction has respectively indexes $i$ and $j$ for directions $x$ and $y$. If we want to calculate the height function for the cell at $(i, j)$, it would be:

$$
h_{i, j}=\sum_{j^{*}=j-3}^{j^{*}=j+3} C_{i, j} \Delta x
$$




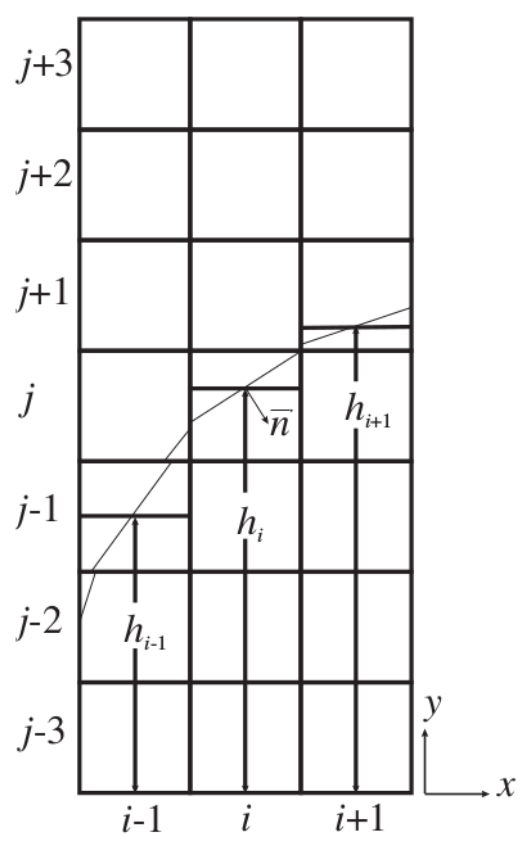

Figure 2.3: Simple 3x7 stencil for the calculation of $h$, from Afkhami and Bussmann [41]

which requires 7 cells in the $y$ direction. The analytical definition of the curvature for a nearly horizontal interface in this case is :

$$
\kappa=\frac{\partial_{y}^{2} h_{i}}{\sqrt{1+\left(\partial_{y} h_{i}\right)^{2}}}
$$

which needs at least a 3 cells stencil in the $x$ direction to be solved using a second order discretization, as:

$$
\kappa=\frac{\left(h_{i-1}-2 h_{i}+h_{i+1}\right) / \Delta x^{2}}{\left.\sqrt{1+\left[\left(h_{i+1}-h_{i-1}\right) /(2 \Delta x)\right.}\right]^{2}} .
$$

the method can be extended to whichever order required and has some important advantages. In a first place, the method improves the robustness of VOF, reducing the formation of small surface fragments (like in CSF). Furthermore, this method is tight-coupled with the surface force term $\mathcal{F}$, hence minimizes the propagation of numerical noise over the surfaces. 


\subsection{Overview of the methods used within ParisSim- ulator}

In this section, the main features used in ParisSimulator are discussed and presented. ParisSimulator implements many of the methods and ideas discussed above and depending of the case studied some methods are more stable or suitable for better physical predictions.

The code is written in Fortran90 (aside from few function and external interfaces in $\mathrm{C}++$ ) and uses a Cartesian staggered mesh for the finite volume discretization of a prismatic domain in order to improve the code parallelization and performances. The parallelization is performed using a homogeneous sub-domain decomposition (all sub-domain are perfect and equal prisms).

Both FT and VOF methods are implemented in ParisSimulator, but obviously in this thesis only the VOF method has been used due to the incapability of the FT to handle complex topology changes. The advection of the surface is done using a modified version of the PLIC algorithm, called the Calcul d'Interface Affine par Morceaux (CIAM). The surface tension contribution are calculated using a CSF method using HF (in its version by Popinet [42]) for computing the curvature.

The spatial discretization of the momentum advection is performed using the QUICK algorithm, while the diffusive flux is solved using a second-order central scheme.

The temporal solution is performed using the Chorin projection method in its second order predictor-corrector formulation. The internal Poisson solver with over-relaxation for the pressure iteration is preferred to the multigrid as it has showed more stability in preliminary studies (also when compared with the library HYPRE). For this reason, one of the tools developed within this thesis aims to improve the time-consumption of the Poisson solver of ParisSimulator using GPU devices and will be briefly described in Appendix A. It should be noted that although this solver was successfully developed and held very positive results, it came at a stage of the thesis when no HPC-GPU hours were available, therefore no applications to the simulations performed within the present thesis was possible. On the other hand, it offers an extremely valuable tool for high density-ratio flows, where the convergence speed of the Poisson solver is often slow. 


\section{References}

[1] Fuster, Daniel and Popinet, Stéphane. "An all-Mach method for the simulation of bubble dynamics problems in the presence of surface tension". In: Journal of Computational Physics 374 (2018), pp. 752-768. DOI: $10.1016 / j \cdot j c p .2018 .07 .055$.

[2] Pitsch, H. and Desjardins, Olivier. "Detailed Numerical Investigation of Turbulent Atomization of Liquid Jets". In: Atomization and Sprays 20.4 (2010), pp. 311-336. DOI: 10.1615/atomizspr.v20.i4.40.

[3] Desjardins, Olivier, Moureau, Vincent, and Pitsch, Heinz. "An accurate conservative level set/ghost fluid method for simulating turbulent atomization". In: Journal of Computational Physics 227.18 (2008), pp. 8395-8416. DOI: $10.1016 / j . j c p .2008 .05 .027$.

[4] "On simulating primary atomization using the refined level set grid method". In: Atomization and Sprays 21.4 (2011), pp. 283-301. DOI: 10.1615/AtomizSpr. 2011002760.

[5] Desjardins, Olivier. NGA. 2012.

[6] Popinet, Stéphane. Gerris code. 2010.

[7] Popinet, Stéphane. Basilisk code. 2014.

[8] Agbaglah, Gilou et al. "Simulation parallèle adaptative octree d'écoulements multiphasiques par suivi d'interface de type volume de fluide". In: Comptes Rendus - Mecanique 339.2-3 (2011), pp. 194-207. DOI: $10.1016 / j$.crme.2010.12.006.

[9] Ling, Y., Fuster, D., Tryggvason, G., and Zaleski, S. "A two-phase mixing layer between parallel gas and liquid streams: Multiphase turbulence statistics and influence of interfacial instability". In: Journal of Fluid Mechanics 859 (2019), pp. 268-307. DOI: 10.1017/jfm. 2018. 825.

[10] Jarrahbashi, D., Sirignano, W. A., Popov, P. P., and Hussain, F. "Early spray development at high gas density: hole, ligament and bridge formations". In: Journal of Fluid Mechanics 792 (2016), pp. 186-231. DOI: 10.1017/jfm. 2016.71.

[11] Hasslberger, Josef, Ketterl, Sebastian, Klein, Markus, and Chakraborty, Nilanjan. "Flow topologies in primary atomization of liquid jets: A direct numerical simulation analysis". In: Journal of Fluid Mechanics 859 (2019), pp. 819-838. DOI: 10.1017/jfm.2018.845. 
[12] Ménard, T, Tanguy, S, and Berlemont, A. "Coupling level set/VOF/ghost fluid methods: Validation and application to 3D simulation of the primary break-up of a liquid jet". In: International Journal of Multiphase Flow 33.5 (2007), pp. 510-524. DOI: 10.1016/j.ijmultiphaseflow.2006.11.001.

[13] CORIA. Archer code. 2005.

[14] Zaleski, S, Tryggvason, G, and Scardovelli, R. ParisSimulator code. 2013.

[15] Tryggvason, Grétar, Scardovelli, Ruben, and Zaleski, Stéphane. Direct numerical simulations of gas-liquid multiphase flows. Cambridge University Press, 2011.

[16] Popinet, Stéphane. "Numerical Models of Surface Tension". In: Annual Review of Fluid Mechanics 50.1 (2017), pp. 49-75. DOI: 10 . 1146 / annurev-fluid-122316-045034.

[17] Scardovelli, Ruben and Zaleski, Stéphane. "Direct Numerical Simulation of Free-Surface and Interfacial Flow". In: Annual Review of Fluid Mechanics 31.1 (1999), pp. 567-603. DOI: $10.1146 /$ annurev . fluid. 31.1 .567$.

[18] Gorokhovski, Mikhael and Herrmann, Marcus. "Modeling Primary Atomization". In: Annual Review of Fluid Mechanics 40.1 (2008), pp. 343366. DOI: 10.1146/annurev.fluid.40.111406.102200.

[19] Chorin, Alexandre Joel. "Numerical solution of the Navier-Stokes equations". In: Mathematics of Computation 22 (1968), pp. 745-762. DOI: $10.2307 / 2004575$.

[20] Kim, J. and Moin, P. "Application of a fractional-step method to incompressible Navier-Stokes equations". In: Journal of Computational Physics 59.2 (1985), pp. 308-323. DOI: 10 . 1016/0021-9991(85 ) 90148-2.

[21] Leonard, B. P. "A stable and accurate convective modelling procedure based on quadratic upstream interpolation". In: Computer Methods in Applied Mechanics and Engineering 19.1 (1979), pp. 59-98. DOI: 10 . 1016/0045-7825 (79) 90034-3.

[22] Shu, Chi Wang and Osher, Stanley. "Efficient implementation of essentially non-oscillatory shock-capturing schemes". In: Journal of Computational Physics 77.2 (1988), pp. 439-471. DOI: 10 . $1016 / 0021-$ 9991 (88)90177-5. 
[23] Liu, Xu-Dong, Osher, Stanley, and Chan, Tony. "Weighted Essentially Non-oscillatory Schemes". In: Journal of Computational Physics 115.1 (1994), pp. 200-212. DOI: 10.1006/jcph.1994.1187.

[24] Bell, John B, Colella, Phillip, and Glaz, Harland M. "In this paper we develop a second-order projection method for the incom- pressible Navier-Stokes equations". In: Journal of Computational Physics 283 (1989), pp. 257-283.

[25] Leer, Bram van and Powell, Kenneth G. Introduction to Computational Fluid Dynamics. 2010. DOI: 10.1002/9780470686652 . eae048.

[26] Vorst, H. A. van der. "Bi-CGSTAB: A Fast and Smoothly Converging Variant of Bi-CG for the Solution of Nonsymmetric Linear Systems". In: SIAM Journal on Scientific and Statistical Computing 13.2 (2005), pp. 631-644. DOI: 10.1137/0913035.

[27] Briggs, William L., Henson, Van Emden, and McCormick, Steve F. "A Multigrid Tutorial, Second Edition". In: A Multigrid Tutorial, Second Edition December 2015 (2011). DOI: 10.1137/1.9780898719505.

[28] Unverdi, S O and Tryggvason, G. "A front-tracking method for viscous, incompressible, multi-fluid flows". In: J. Comput. Phys. 100 (1992), pp. 25-37.

[29] Tryggvason, G. et al. "A Front-Tracking Method for the Computations of Multiphase Flow". In: Journal of Computational Physics 169.2 (2001), pp. 708-759. DOI: 10.1006/jcph.2001.6726.

[30] Lu, Jiacai and Tryggvason, Gretar. "Direct numerical simulations of multifluid flows in a vertical channel undergoing topology changes". In: Physical Review Fluids 3.8 (2018), pp. 1-20. DOI: 10.1103 / PhysRevFluids.3.084401.

[31] Lin, Shengxiang, Lu, Jiacai, Tryggvason, Gretar, and Zhang, Ying. "A numerical study of oscillation induced coalescence in bubbly flows". In: Physics of Fluids 30.12 (2018). DOI: 10.1063/1.5059558.

[32] Hirt, C.W W and Nichols, B.D D. "Volume of fluid (VOF) method for the dynamics of free boundaries". In: Journal of Computational Physics 39.1 (1981), pp. 201-225. DOI: 10.1016/0021-9991(81)90145-5.

[33] Sussman, Mark. "A level set approach for computing solutions to incompressible two-phase flow". In: Journal of Computational Physics 114.1 (1994), pp. 146-159. DOI: 10.1006/jcph.1994.1155. 
[34] Noh, W.F. and Woodward, P. SLIC (simple line interface calculation). [Usable in 1, 2, or 3 space dimensions]. Tech. rep. Livermore, CA: Lawrence Livermore National Laboratory (LLNL), 1976. DOI: 10. 2172/7261651.

[35] Hirt, C. W. and Nichols, B. D. "Volume of fluid (VOF) method for the dynamics of free boundaries". In: Journal of Computational Physics 39.1 (1981), pp. 201-225. DOI: $10.1016 / 0021-9991$ (81)90145-5.

[36] Youngs, D. "Them dependent multimaterial flow with large fluid distribution". In: Numer. Methods Fluid D 24.273-285 (1982).

[37] "Piecewise Linear Interface Calculation". In: Comptes Rendus Academie des Sciences, Serie II: Mecanique, Physique, Chimie, Astronomie 320.8 (1995), pp. 391-396.

[38] Scardovelli, Ruben and Zaleski, Stephane. "Interface reconstruction with least-square fit and split Eulerian-Lagrangian advection". In: International Journal for Numerical Methods in Fluids 41.3 (2003), pp. 251-274. DOI: 10.1002/fld.431.

[39] Brackbill, J. U., Kothe, D. B., and Zemach, C. "A continuum method for modeling surface tension". In: Journal of Computational Physics 100.2 (1992), pp. 335-354. DOI: 10.1016/0021-9991(92) 90240-Y.

[40] Fedkiw, Ronald P., Aslam, Tariq, Merriman, Barry, and Osher, Stanley. "A Non-oscillatory Eulerian Approach to Interfaces in Multimaterial Flows (the Ghost Fluid Method)". In: Journal of Computational Physics 152.2 (1999), pp. 457-492. DOI: 10.1006/jcph.1999.6236.

[41] Afkhami, S. and Bussmann, M. "Height functions for applying contact angles to 3D VOF simulations". In: International Journal for Numerical Methods in Fluids 61.8 (2009), pp. 827-847. DOI: 10.1002/fld. 1974.

[42] Popinet, Stéphane. "An accurate adaptive solver for surface-tensiondriven interfacial flows". In: Journal of Computational Physics 228.16 (2009), pp. 5838-5866. DOI: 10.1016/j.jcp.2009.04.042. 



\section{Chapter 3}

\section{Treatment of the inflow boundary conditions}

In this chapter, the inflow boundary conditions used in this work will be discussed and presented. The synthetic turbulence inflow boundary condition from [1] will be presented, and will generally be referred to as Synthetic Boundary Condition (or SBC). Finally, the procedure for generating the data required for the Mapped Boundary Condition (called MBC) will be discussed, with details on the methods used for the calculation and the implementation. While, at first, the SBC method will be used in Chapter 5, the comparison with the SBC and MBC (adequately tuned for being comparable physically) will be presented in Chapter 7

\subsection{Synthetic inflow boundary conditions}

In this section, a discussion over the synthetic turbulent inlet boundary conditions and their usage in DNS simulation is addressed. First a review of the available suitable methods will be provided and then a description of the method proposed by Klein et al. [1] will be discussed.

\subsubsection{Review of available methods}

Various approaches have been proposed to simulate a synthetic turbulent inflow boundary condition. Klein et al. [1] proposes to use a linear-non recursive 
filter, based on the hypothesis of homogeneous turbulence, to filter a random signal. The result of the filtered procedure is a correlated field in both time and space. An interesting and useful modification to this procedure was provided by Hoepffner et al. [2], where an auto-regressive procedure was used to determine the filter coefficients. This method requires an a-priori knowledge of the turbulence behavior that will be replicated by the synthetic turbulence generator, but it offers a higher flexibility in setting both the time and the lengthscale, allowing to inject vortices of different size and with different dynamic behaviorr.

Perret et al. [3] used Proper Orthogonal Decomposition (POD) to extract the turbulence coherent structures from experimental data. A non-linear Reduced Order Model is then generated using Galerkin projection, which has been widely used in CFD thanks to its capabilities to replicate the non-linear behaviour of turbulence. Still, as for [2], accurate and verified data are required in order to reproduce the injected turbulence behaviour, which may not always been available or adequate to fit the required time or space scales.

Lee et al. [4] used a Fourier harmonics based method to reproduce the behaviour of a certain portion of the energy spectra. Each Fourier mode is individually computed and finally combined with the others through the use of a random phase. This method shows interesting results for isotropic decaying turbulence, but it is worth notice that the maximum change of the random phase and the time needed by the phase to actually change may influence the turbulence behaviour, leading to possible non-physical results [5].

Some interesting reviews of the methodologies for the generation of this synthetic turbulence (not all of them feasible for this test case) are given in [2, $5,6]$. Nevertheless, it has been proven by Druault et al. [7] that uncorrelated velocity fluctuations decays rapidly and are not able to maintain turbulence. Therefore the usage of a synthetic inlet condition correlated in time and space is mandatory to reproduce the behaviour of the nozzle outlet velocity field.

As the effects of the turbulence may only be assessed after a significant penetration, due to the required time for shear stresses to participate in generating the surface instabilities on the spray core [8], no comparison on the effects of different methodologies have been made, although it may represent an interesting analysis for future developments. Finally, the method proposed by [1] has been used, due to the promising results obtained in [9]. 


\subsubsection{Implementation of the synthetic turbulence inlet bound- ary conditions}

As already stated previously, the simulation of an inlet turbulence condition for the jet is of fundamental importance to reproduce the real behaviour of atomization. In the simulations performed in this thesis, the simulation domain is prismatic with cubical cells (according to the solver configurations [10]), with a circular spray incoming from the $-\hat{x}$ direction, with a positive velocity component in the $\hat{x}$ direction. In this frame, the turbulence has to take into account the following features:

- The turbulence has to be distributed on all of the velocity components, with a pre-determined velocity module.

- The turbulence has to be zero-mean in time on all the components, so that the resulting mean component is the original and stationary velocity field input.

- The turbulence distribution has to be continuous in time and space, at all time steps.

To accommodate all this necessities, the original algorithm implemented by Klein et al. [1] has been chosen as a methodology. This method already showed its capabilities for both planar [1] and circular [9] jets, although no analysis on the physical consequences of the tunable parameters of this method have been found in literature.

This method is based on the Reynolds decomposition [11] on the inlet velocity field:

$$
\mathbf{U}\left(x_{0}, t\right)=\langle\mathbf{U}\rangle\left(x_{0}\right)+\mathbf{u}\left(x_{0}, t\right)
$$

where the effective velocity $\mathbf{U}$ is decomposed into a mean field $\langle\mathbf{U}\rangle$ and a fluctuating component $\mathbf{u}$ that is based on a random component. This kind of decomposition for the inlet boundary condition allows to model the behavior of $u$, so that a one point correlation can be imposed, as well as a limitation on the energy level associated with the fluctuating component. In order to determine the fluctuation, it might be decomposed into the product of two factors, namely the Reynold stress tensor and random velocity field, continuous in time and space. Consequently, equation (3.1) can be rewritten as:

$$
\mathbf{u}\left(x_{0}, t\right)=\langle\mathbf{U}\rangle\left(x_{0}\right)+\mathbf{A} \cdot \mathbf{u}\left(x_{0}, t\right)
$$


A is a correlation tensor of coefficients $a_{i j}$, that is designed to match the desired Reynold Stress Tensor from equation (3.2), as demonstrated in [12]. The non-zero Tensor components are:

$$
\begin{aligned}
& a_{11}=\sqrt{R_{11}}, \\
& a_{21}=R_{11} / a_{11}, \\
& a_{22}=\sqrt{R_{22}-a_{21}{ }^{2}}, \\
& a_{31}=R_{31} / a_{11}, \\
& a_{32}=R_{32}-a_{21} a_{31} / a_{22} \\
& a_{33}=\sqrt{R_{33}-a_{31}{ }^{2}-a_{32}{ }^{2}} .
\end{aligned}
$$

Where $R$ is the autocorrelation function, that may be obtained from internal nozzle flow simulations. In order to determine the fluctuating component, a linear non-recursive Finite Impulse Response (FIR) filtering procedure is applied to a random field, as:

$$
u=\sum_{n=-N}^{N} b_{n} r_{n+m}
$$

where $r$ is the random field and $b_{n}$ are the filter coefficients. While the determination of $r$ is a straight forward procedure, it is important to have $\bar{r}_{m}=0$ and $\bar{r}_{m} r_{m}=1$ in order to obtain a two-point correlation.

The determination of the filter coefficients, can be derived through the definition of the autocorrelation function of equation (3.4) as:

$$
\frac{\overline{u_{m} u_{m+k}}}{\overline{u_{m} u_{m}}}=\frac{\sum_{j=-N+k}^{N} b_{j} b_{j-k}}{\sum_{j=-N}^{N} b_{j}^{2}} .
$$

Once the statistical properties of the random field are matched, equation (3.5) can be used to find the filter coefficient values if an autocorrelation function (equation (3.5) left-hand side) is given; to overcome this obstacle, Klein et al. [1] proposed to use the 1D spatial autocorrelation provided by Batchelor in [13]:

$$
R_{u u}(d, 0,0)=\frac{\overline{u_{m} u_{m+k}}}{\overline{u_{m} u_{m}}}=\exp \left(-\frac{\pi d^{2}}{4 L^{2}}\right)
$$


where $d$ is the distance vector (characteristic of the specific problem) and $L$ is the turbulent length scale, for which specific values may be found in [9] for cylindrical jet applications. Equation (3.6) can be combined with (3.5) and solved for $b_{j}$, obtaining:

$$
b_{k}=g_{k} / \sum_{j=-N}^{N} g_{j}^{2} \quad \text { and } \quad g_{i}:=\exp \left(-\frac{\pi k}{2 n^{2}}\right)
$$

where $n=L / \Delta x$ is the discrete length scale (with $\Delta x$ being the mesh size, uniform in the three directions in the present study).

The filtering operation can be easily convoluted in three dimensions and need no further explanations (more details and suggestion on the filter implementation can be found in [1]).

\subsubsection{Velocity Inlet Profile and Turbulence Distribution}

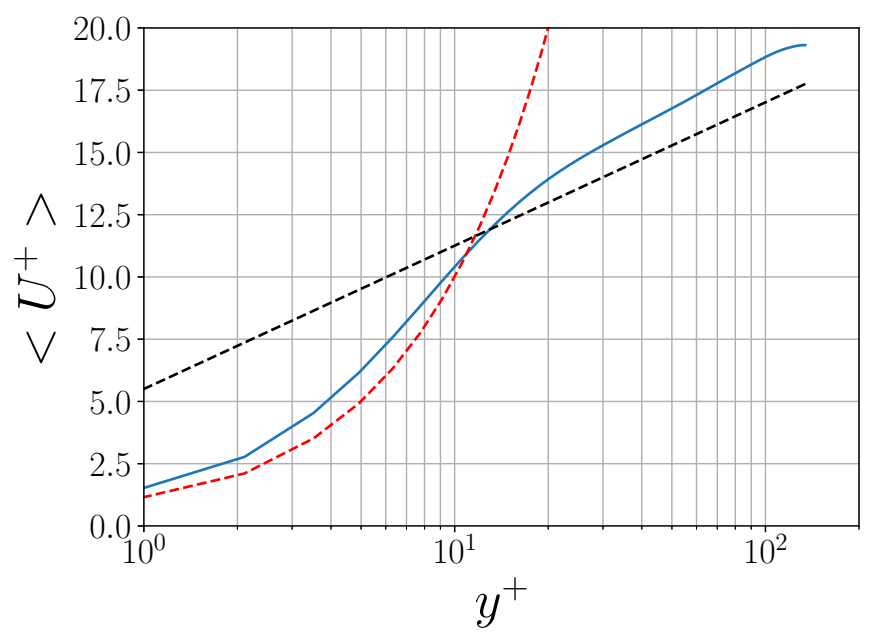

Figure 3.1: Axial velocity profile in a turbulent pipe, from [14]

The usual approach in DNS simulations of sprays [9, 15] is to use a flat-top inlet velocity profile, as the actual profile developed inside the injector is still being discussed and strongly depends on the injector geometry. Therefore, as many applications in different sectors (automotive, energy, aerospace, industrial) have different injection system, it is reasonable to use a fully developed 


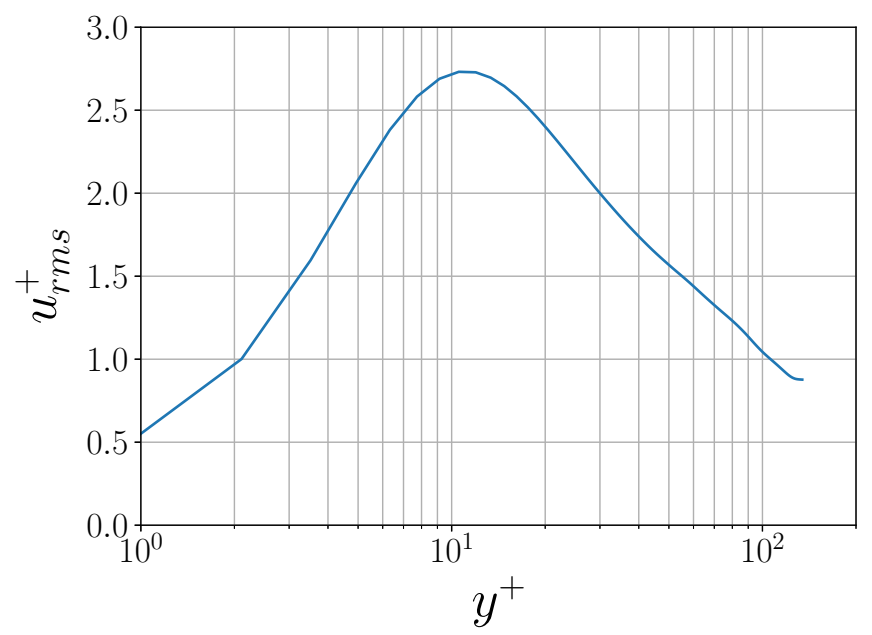

Figure 3.2: Axial velocity fluctuation profile in a turbulent pipe, from [14]

turbulent pipe flow as turbulent source for the spray. In this thesis a turbulent pipe profile has been used both for the radial distribution of the velocity, as well as for the turbulence distribution. While for direct comparison with the MBC cases it is preferable to use them directly to extract these profiles, for an assessment over this boundary condition literature data have been used. Many works are available on both experimental and numerical studies of pipe flow turbulence at moderate Reynolds number $[14,16]$. If the mass flow rate is fixed, the mean velocity profile $\mathbf{U}\left(x_{0}\right)$ impose a significantly higher velocity at the centerline for the case accounting for the wall effects as compared to a typical flat-top profile. For this reason, In the course of this thesis, there will be a differentiation over the bulk Reynolds $R e_{b}$ and the centerline Reynolds $R e_{c}$, as they change significantly and affect many feature of the spray formation. Figure 3.1 shows in blue the velocity profile used in the study of this boundary condition, derived by the work of [14], in red the linear region $\left(y^{+}=y^{+}\right)$, while in dashed black the logarithmic region, defined by:

$$
U^{+}=\frac{1}{k} \log \left(y^{+}\right)+B
$$

with $k=0.4$ and $B=5.5$. The $y^{+}$is defined as:

$$
y^{+}=\frac{y u_{\tau}}{\nu}
$$


where $y$ is the wall distance, $u_{\tau}$ is the shear velocity and $\nu$ is the kinematic viscosity. The lack of agreement between the work in [14] and the theoretical behavior has several reasons. In a first place, the limited $R e$ at which the data are obtained may have influenced the result. On the other hand, the coefficient in Equation (3.8) are obtained from [11] and not directly from the author.

The fluctuating velocity component $\left(u\left(x_{0}, t\right)\right)$ distribution has been set so that it will comply with the behavior illustrated in Figure 3.2. While it will be discussed more in details in the next section, it is necessary to provide a early discussion over the differences between the typical lengthscales of the flow in a pipe (or nozzle/duct) and the atomizing spray scale. In the spray near field, as it will be addressed, there are two different integral scales, depending if we consider the transient or the statistical stationary flow. In the first case, the largest scale is the toroid-vortex behind the spray tip, which has roughly a radius which is twice the nozzle's one. In statitstical stationary conditions, the integral lengthscale is roughly the spray diameter. For fully developed pipe flow, such a scale is usually assumed to be $10 \%$ of the diameter. This discrepancy in integral scales between the internal and external flow force a substantial difference also in the smallest scales, such as $\eta$, which can reflect in the $y^{+}$being substantially smaller than the DNS cell size. Hence the profile in Figure 3.2. will likely been interpolated, therefore no exact solution is required. Furthermore, the most important structures to be inserted in the simulation by the nozzle are the large ones, as the smaller will be quickly smoothed by the surface tension.

\subsection{Mapped boundary conditions}

The idea behind the MBC method is to generate an accurate simulation of the nozzle flow, so that the larger structures can be captured. Larger turbulent structures are, in fact, the ones which have the most energy content and therefore the ones that are responsible for generate instabilities and breakup. For reasons that will be clear in this section, an accurate representation of the smallest structures is indeed unnecessary due to the difference in the characteristic lengthscale between the nozzle flow and the atomizing spray. Hence, a LES simulation has been used to produce a useful boundary condition for the spray DNS. The simulation has been validated and analyzed in detail as it will be demonstrated in the next subsections. 


\subsubsection{Methodology and analysis for the LES simulation}

In order to generate a set of reliable boundary condition, a fully turbulent periodic pipe flow has been simulated using a LES approach. The domain has been designed so that all the hairpins and turbulent structures generated within the flow can freely develop. The crucial parameter in designing the simulation is the ratio $L_{p} / D_{n}$ where $L_{p}$ is the pipe length and $D_{n}$ is the pipe diameter. As extensively addressed in literature $[14,16,17]$ this ratio is crucial to capture the larger structures, which are inherently the ones with the higher energy content and, therefore, the ones that are most likely to affect the spray atomization. Furthermore, the influence of the duct length and the importance of the larger size structures have been analyzed in [18] on a long pipe $\left(L_{p}=15 D_{n}\right)$ at $R e=24580$, confirming that an insufficient length leads to a overall inaccuracy in capturing the larger turbulent structures, as already demonstrated by [19] for channel flows. Many studies have used different ratios, especially for DNS studies. Wu and Moin [20], again, used a $L_{p} / D_{n}=$ 7.5 , which reportedly was enough to allow the formation of the larger structure at $R e_{b}=5300$. This specific $R e_{b}$ is close to the one used in the current analysis and, as confirmed in [16] for a $R e_{b}=5300$, this $L_{p} / D_{n}$ ratio is enough to account for all the scales of motion. Therefore, in the current work, $L_{p} / D_{n}=8$ has been chosen for the LES calculation as a safety ratio, following the analysis of the results in literature. The solver pisoFOAM is used, based on the PISO algorythm Issa [21], to solve the following governing equations:

$$
\begin{gathered}
\frac{\partial \widetilde{u_{i}}}{\partial t}+\frac{\partial \widetilde{u_{i} u_{j}}}{\partial x_{j}}=-\frac{1}{\rho_{l}} \frac{\partial \widetilde{p}}{\partial x_{i}}+\nu \nabla^{2} u_{i} \\
\frac{\partial \widetilde{u_{i}}}{\partial \widetilde{x_{i}}}=0
\end{gathered}
$$

where $\widetilde{u_{i}}$ is the filtered velocity field, $\rho_{l}$ is the liquid density (constant) and $\widetilde{p}$ is the filtered pressure field. The sub grid scale model chosen is the Wall-Adaptive Local Eddy-viscosity (WALE) where the term $\widetilde{u_{i} u_{j}}$ in Equation (3.10a) is rewritten as:

$$
\widetilde{u_{i} u_{j}}=\tau_{i j}+\widetilde{u_{i}} \widetilde{u_{j}}
$$

where $\tau_{i j}$ is the residual (or sub-grid) stress tensor, which is modeled according to Nicoud and Ducros [22].

Finally, the simulation is performed at a fixed time-step of $20 \mathrm{~ns}$ for a time equal to 60 washouts in order to allow for reliable statistics. Although 
a more detailed analysis and discussion about washouts will be provided in Chapters 5 to 7 , for now it is sufficient to think about it as the time required for a liquid infinitesimal element to cross the whole stream direction.

\section{Validation and analysis}

In order to validate the simulation outcome, the results have been compared to literature data from Eggels et al. [14] for both, the average velocity and the root mean squared components in Figures 3.3 and 3.4.

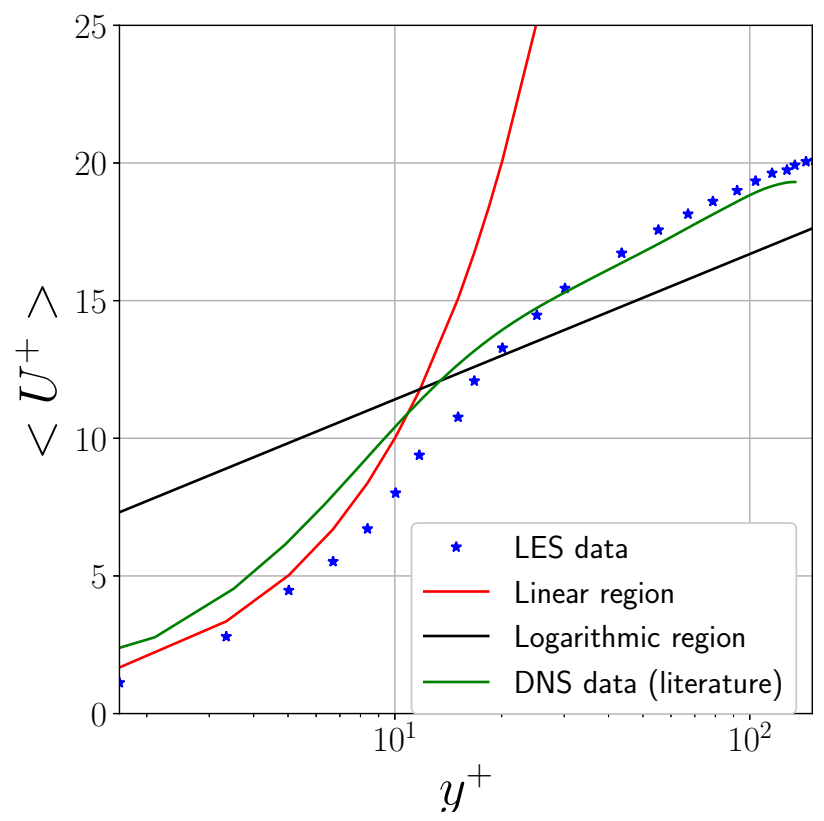

Figure 3.3: Wall law, plotted using the dimensionless mean velocity.

Figures 3.3 and 3.4 shows a good agreement between the LES data and the DNS data, where only an error lower than $5 \%$ is experienced. This behavior inherently states that the simulation is resolved (at least from a statistical standpoint) at the wall as this kind of deviation is quite typical in similar works [20].

Finally, these results lead to the discussion over the mesh size difference between the LES and the DNS simulation. The necessity to resolve the wall shear forces a mesh refinement in the LES simulation, while the DNS, using 


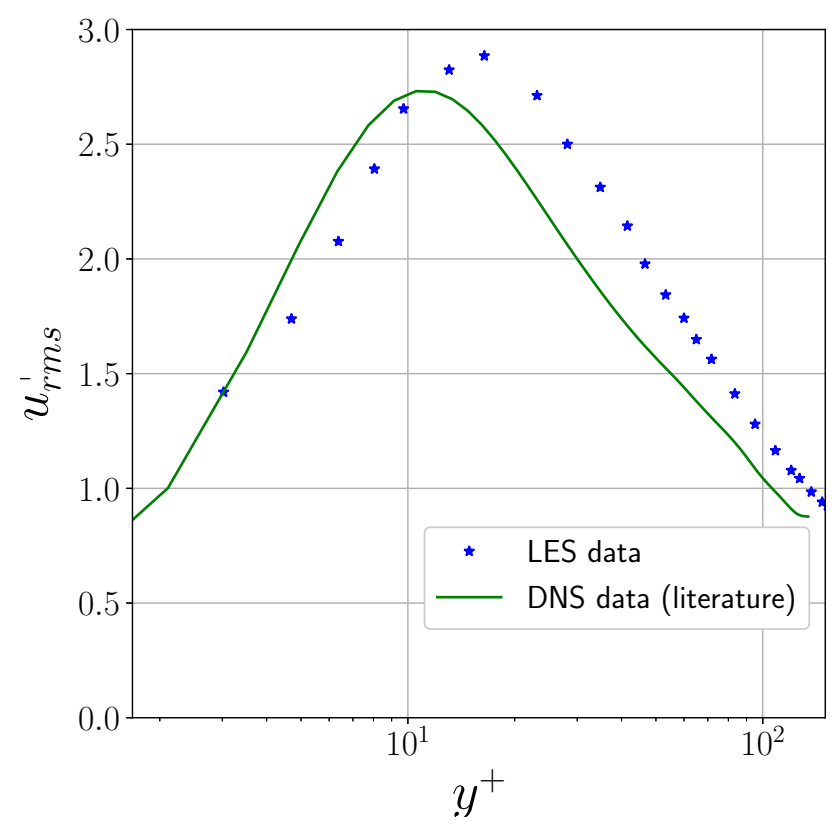

Figure 3.4: Wall-law for the velocity root mean squared velocity component.

a cuboid Cartesian grid, has a constant mesh size. As an interpolation will be mandatory in order to adapt the solution to the coarser DNS mesh, this will also imply that some frequencies will be filtered out. While it remains to be proven that this is not a major influence on the atomization process (especially in the generation of smaller droplets), it is ideal for the present comparison between MBC and SBC.

What discussed above, lead to an obvious conclusion which is worth discussing. In fact, while for smaller scales the hypothesis of homogeneous turbulence may be acceptable (as an extension of the local isotropy hypothesis formulated by Kolmogorov [24]), it is most likely not the case for larger scales of motion, close to the integral length scale. Figure 3.5 displays the turbulent structures detected using the Q-criterion algorithm by [23] colored by the local vorticity. This snapshot (performed after 30 washouts) clearly shows a major axial orientation of the turbulent structures, which are advected by the main flow, suggesting an anisotropic action of the velocity components. 


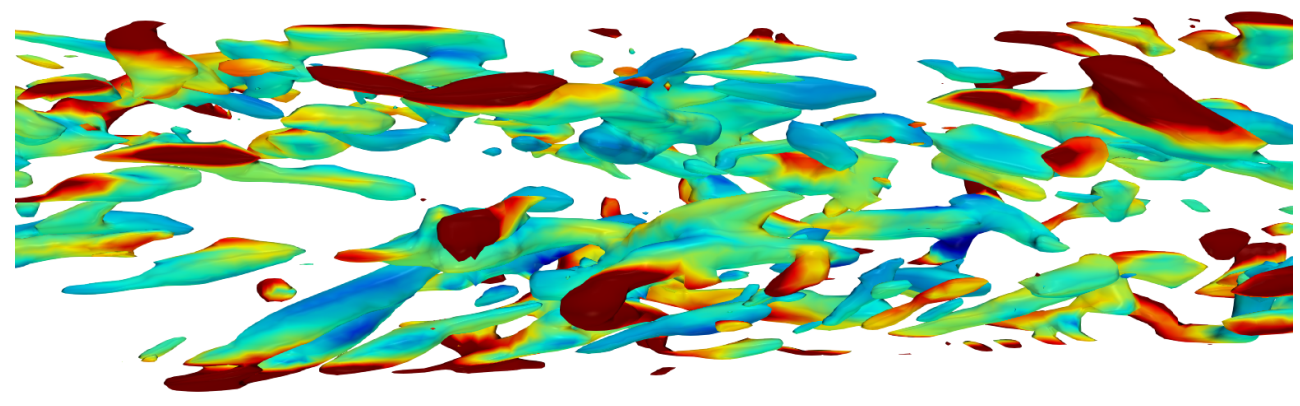

\section{$|\omega|[1 / s]$}

$100 \quad 2.5 e+6 \quad 5 e+6 \quad 7.5 e+6 \quad 1 e+7$

Figure 3.5: Turbulent structures captured by the $Q$ criterion as described in [23].

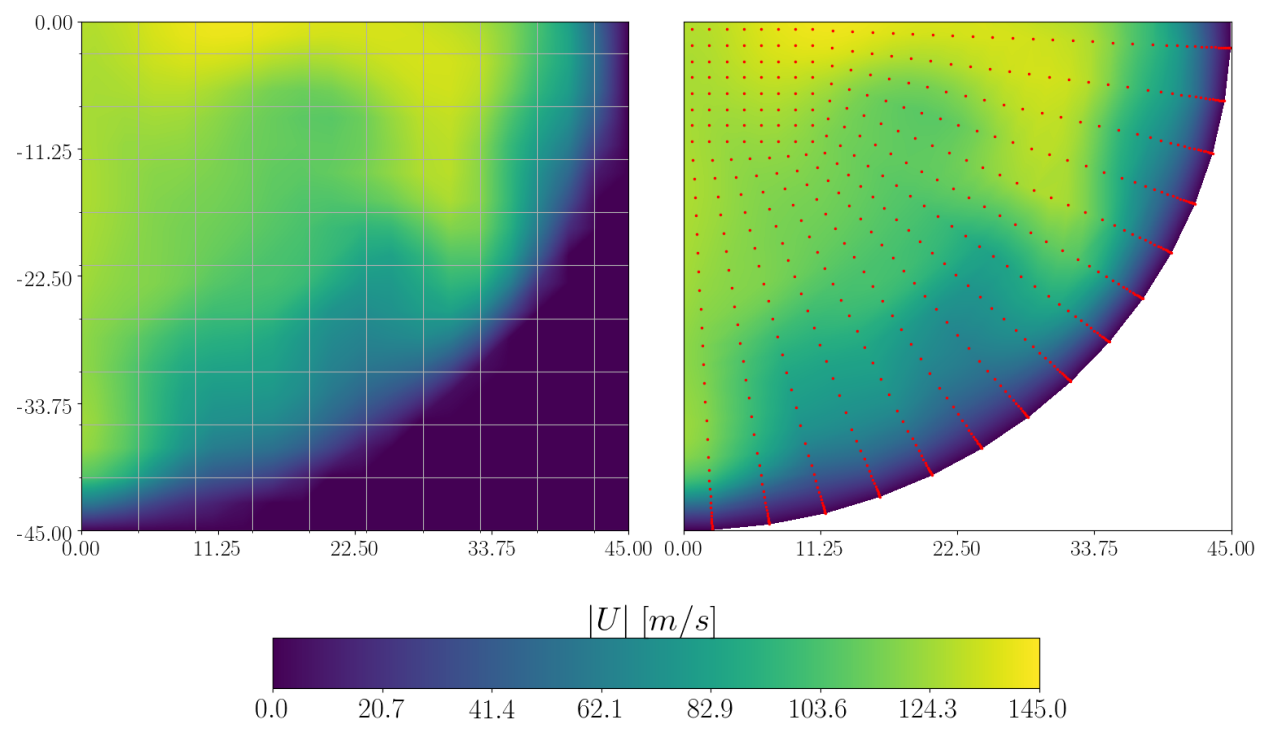

Figure 3.6: Inlet boundary condition for the $M B C$ case. On the left the interpolated result to be fed into ParisSimulator and the mesh, on the right the LES corresponding result and the mesh points in red. All the axis are expressed in $\mu \mathrm{m}$. 


\section{Velocity interpolation from LES to DNS simulation}

Once the LES results have been validated, the results can be interpolated into a cartesian mesh for usage in ParisSimulator. As previously observed, the larger structures are faithfully reproduced but is worth address the wall behavior. As a matter of fact, the wall spacing in the LES simulation is $\delta_{L E S}=0.45 \mu \mathrm{m}$ (and increase exponentially), which is more than five times smaller than the cell size $d x$ in the DNS case. For this specific reason, it is important to notice that the wall behavior, as well as the smallest turbulent structures of the LES, will be neglected in the DNS. In order to reproduce the mean flow (Figure 3.3) it is not significant, as a linear region can easily be reproduced with only 2 points. On the other hand, the velocity fluctuations, especially at their peak (Figure 3.4) will be attenuated.

As previously mentioned, while this simplification may represent an incomplete representation of the LES results, it improves once again the comparability between the MBC and SBC simulations, where the effect of the most turbulent region is under-resolved. Furthermore, a case can be made [25] that surface tension inherently dumps the effect of smaller eddies, largely developed in the near-wall region. In fact, once the liquid in the wall region outgoes the nozzle, it immediately starts producing shear with the calm gas phase, hence generating forces that are orders of magnitudes larger than the ones induced by the wall turbulence. As it will be discussed later, however, the shape of the perturbations developed in the liquid core are of primary importance in the generation of shear and consequently in breakup. This perturbations are indeed induced by the radial and the tangential components of the velocity in the nozzle, hence accurately reproducing the turbulence behavior is of fundamental importance. In Chapter 7 a detailed analysis will be provide, proving how the most important feature to capture in nozzle flows is the large structure behavior. Finally, a cartesian mesh resolved up to $\delta_{L E S}$ is unfeasible even for state of art DNS simulations Ling et al. [26].

Finally, this effect, while worth mentioning, is considered to be irrelevant for the present study.

\subsubsection{Turbulent intensity and lengthscale extracion for the SBC case}

The LES simulation can also be analyzed for extracting the $I$ and $L$ parameters for the synthetic turbulent boundary conditions for the SBC case. The derivation of the two has been made rigorously in this case, as it is impor- 
tant to limit the differences between SBC and MBC cases only to quantifiable causes.

The theoretical formulation for the Integral length scale relies in its definition of being the lengthscale characteristic of larger turbulent structures. The same definition can be applied to the so-called turbulent lengthscale $L$. The equation defining it is:

$$
L(r, t)=\int_{0}^{2 \pi} \phi(r, \delta \theta, t) d(\delta \theta)
$$

where $r$ is the radial distance from the pipe center (equal to $D_{n} / 2$ at the wall), $\theta$ is the azimuthal coordinate and $\phi$ is the dimensionless autocorrelation function. As known from literature Pope [11], for wall-bounded flows, $r$ is the direction on which the turbulence intensity varies (as demonstrated in Figure 3.4) while $\delta \theta$ is the distance vector in the direction on which the turbulence is statistically stationary. Therefore, fixing $r$ and varying $\theta$ we can find a number of locations (finite in our discrete analysis) where the turbulence behave similarly over time. This consideration leads the way that if we integrate over $\theta$, a signal average of $\phi(r, \theta, t)$ over time may be a reasonable way to remove the dependency of $L$ from time.

The calculation of the dimensionless autocorrelation function can be done by considering that the signal is indeed periodic when sampled over $\theta$, hence the first and the last value of the autocorrelation need to be equal when the total autocorrelation length is $2 \pi$. Furthermore, being the signal periodic it is always possible to compute the product $u(\theta) u(\theta+\delta \theta)$ with $\delta \theta \in[0,2 \pi[$ simply by concatenating the signal twice. Therefore, the formal definition of the dimensionless autocorrelation function is:

$$
f_{11}(r, \delta \theta, t)=\frac{\langle u(r, \theta, t) u(r, \theta+\delta \theta, t)\rangle}{\left\langle u(r, \theta, t)^{2}\right\rangle}
$$

The computation of Equations (3.12) and (3.13) provides the result showed in Figures 3.7 and 3.8. The function $L(r)$ is finally averaged to produce a single scalar usable for the SBC case. The result produce a value for $L$ of $9.23 \mu \mathrm{m}$, which corresponds to $10.25 \%$ of the nozzle diameter $D_{n}$.

The definition of $I$ mostly used in CFD is:

$$
I=\frac{u_{r m s}}{\langle U\rangle}=0.16 R e_{b}^{-1 / 8}
$$




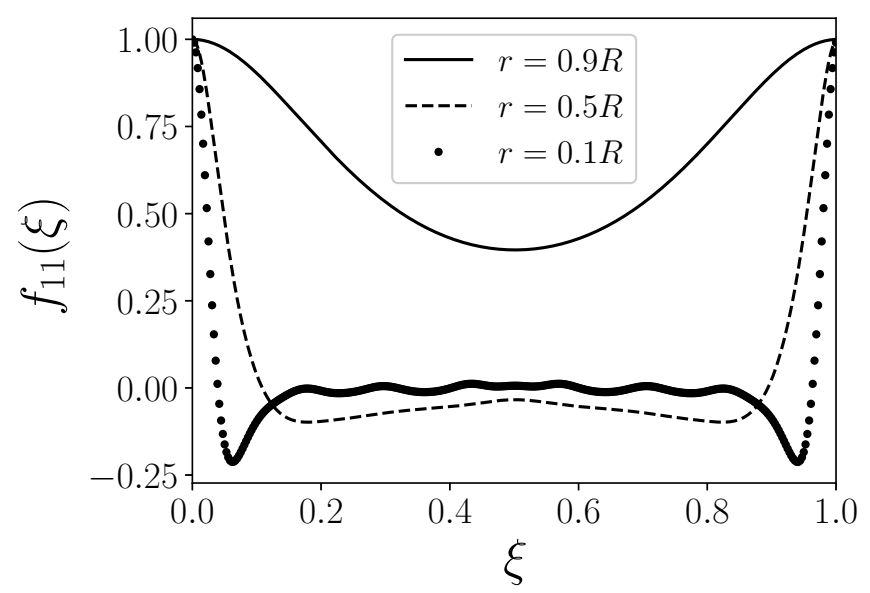

Figure 3.7: Autocorrelation for the LES simulation of pipe flow.

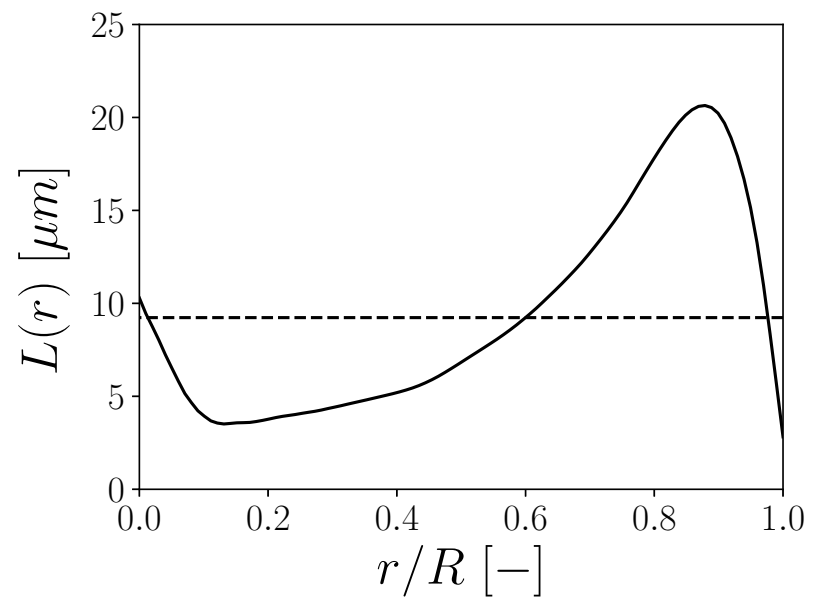

Figure 3.8: Turbulent integral lengthscale as a function of the radial position in the pipe flow.

where $u_{r m s}$ is the root-mean-squared component of the velocity, accounting for all components. The last formulation of Equation (3.14) is the one mostly used in RANS/LES simulation and, in this case gives $5.51 \%$ (for the simulations that will be presented in the following chapter, of dodecane injected in quiescent air). On the other hand, the formal definition was used (second formulation in Equation (3.14)) and averaged over the whole domain, with a result of $5.88 \%$, which is the value that was ultimately used in the SBC 
case.

\subsection{Resume and open questions}

As it has been observed above, adopting the SBC or the MBC has several implication and may be dictated by many factors. In a first place using the SBC is usually a good way to perform a simulation even in case of significant ignorance of the flow within the nozzle. The complexity of modern age applications for nozzle is enormous, with a number of event that may alter substantially the behavior of the nozzle flow. For example, in automotive injectors, the needle transient movement and wobble, complex channel geometry, high injection pression and cavitation are extremely important in determining the flow turbulence within the nozzle. Moreover, when one or more of these conditions occur, it is basically impossible to verify a simulation against experimental data or well established theory of turbulence. While accounting for all these uncertainties is of fundamental importance when the goal of the simulation is to reproduce an experiment, the lack of validation introduces as many uncertainties as the ones given by the flow itself.

These considerations allow to understand why the SBC, in some cases may be preferable. When no validation is available, the simple homogeneous structures generated by the SBC are actually an extremely simple case that neglect all these uncertainties, which is easy to tune using $I, L$ and the velocity profiles and that can be used to generate anisotropic structures as well when Equation (3.3) is adjusted using the provided knowledge on the Reynolds stress tensor. In many cases, this is a preferable choice. Obviously, a significant drawback is that the results will have to be taken as statistical values. In fact, while the Probability Density Function (PDF) for the droplet size distribution may be used to compare against experiments, it is likely that, for example, the rate of total atomized mass (as a percentage over the total mass injected) will not be a comparable value.

While real nozzle cannot always be accurately predict, lab scale experiments may be set in order to reproduce a specific behavior. In this case, an inflow condition as the MBC may actually be recommendable as it catches a number of features that the simple filtering procedure of SBC is not able to capture.

The method discussed above for extracting $I$ and $L$ from the LES simulation, can only aim to reduce differences between the $\mathrm{MBC}$ and the SBC case. In fact, especially in case of strong uncertainty, the tuning performed on the Reynolds stress tensor for the SBC case is limited, hence the synthetic is 
likely to generate isotropic structures, which are substantially different from the anisotropic ones generated by the LES simulation performed for the MBC case.

It will be one of the main goal of this work to understand how to compare this two inflow boundary conditions and what are the main differences produced on the atomization process and the turbulent field.

\section{References}

[1] Klein, M., Sadiki, A., and Janicka, J. "A digital filter based generation of inflow data for spatially developing direct numerical or large eddy simulations". In: Journal of Computational Physics 186.2 (2003), pp. 652-665. DOI: 10.1016/S0021-9991(03)00090-1.

[2] Hoepffner, J., Naka, Y., and Fukagata, K. "Realizing turbulent statistics". In: Journal of Fluid Mechanics 676 (2011), pp. 54-80. DOI: 10. 1017/jfm.2011.32.

[3] Perret, Laurent, Delville, Joël, Manceau, Rémi, and Bonnet, Jean Paul. "Turbulent inflow conditions for large-eddy simulation based on loworder empirical model". In: Physics of Fluids 20.7 (2008). DOI: 10 . 1063/1.2957019.

[4] Lee, Sangsan, Lele, Sanjiva K., and Moin, Parviz. "Simulation of spatially evolving turbulence and the applicability of Taylor's hypothesis in compressible flow". In: Physics of Fluids A 4.7 (1992), pp. 15211530. DOI: $10.1063 / 1.858425$.

[5] Grinstein, F. F. Large Eddy Simulation for Incompressible Flows, An Introduction, 3rd Edition. Vol. 46. 4. Springer Science \& Business Media, 2008, pp. 1021-1023. DOI: 10.2514/1.36015.

[6] Tabor, G. R. and Baba-Ahmadi, M. H. "Inlet conditions for large eddy simulation: A review". In: Computers and Fluids 39.4 (2010), pp. 553567. DOI: $10.1016 / \mathrm{j}$.compfluid.2009.10.007.

[7] Druault, P. et al. "Generation of Three-Dimensional Turbulent Inlet Conditions for Large-Eddy Simulation". In: AIAA Journal 42.3 (2008), pp. 447-456. DOI: $10.2514 / 1.3946$.

[8] Jarrahbashi, D., Sirignano, W. A., Popov, P. P., and Hussain, F. "Early spray development at high gas density: hole, ligament and bridge formations". In: Journal of Fluid Mechanics 792 (2016), pp. 186-231. DOI: $10.1017 / \mathrm{jfm} .2016 .71$. 
[9] Lebas, R., Menard, T., Beau, P.A., Berlemont, A., and Demoulin, François-Xavier. "Numerical simulation of primary break-up and atomization: DNS and modelling study." In: International Journal of Multiphase Flow 35.3 (2009), pp. 247-260. DOI: 10 . 1016 / j . ijmultiphaseflow.2008.11.005.

[10] Ling, Y., Zaleski, S., and Scardovelli, R. "Multiscale simulation of atomization with small droplets represented by a Lagrangian pointparticle model". In: International Journal of Multiphase Flow 76 (2015), pp. 122-143. DOI: 10.1016/j.ijmultiphaseflow. 2015.07. 002.

[11] Pope, Stephen B. Turbulent Flows. 2001. DOI: 10.1088/0957-0233/ 12/11/705.

[12] Lund, T. S., Wu, X., and Squires, K. D. "On the Generation of Turbulent Inflow Conditions for Boundary Layer Simulations". In: Journal of Computational Physics 140 (1998), pp. 233-258. DOI: 10.1006/jcph. 1998.5882.

[13] Davies, T. V. and Batchelor, G. K. The Theory of Homogeneous Turbulence. Vol. 38. 323. Cambridge university press, 2007, p. 64. DOI: $10.2307 / 3609796$.

[14] Eggels, J. G. M. et al. "Fully developed turbulent pipe flow: a comparison between direct numerical simulation and experiment". In: Journal of Fluid Mechanics 268.-1 (2006), p. 175. DOI: 10.1017 / S002211209400131X.

[15] Shinjo, J. and Umemura, Akira. "Simulation of liquid jet primary breakup: Dynamics of ligament and droplet formation". In: International Journal of Multiphase Flow 36.7 (2010), pp. 513-532. DOI: 10 . 1016/j . ijmultiphaseflow.2010.03.008.

[16] El Khoury, George K. et al. "Direct numerical simulation of turbulent pipe flow at moderately high reynolds numbers". In: Flow, Turbulence and Combustion 91.3 (2013), pp. 475-495. DOI: 10.1007/s10494-0139482-8.

[17] Kim, K. C. and Adrian, R. J. "Very large-scale motion in the outer layer". In: Physics of Fluids 11.2 (1999), pp. 417-422. DOI: 10.1063/ 1.869889 .

[18] Wu, Xiaohua, Baltzer, J. R., and Adrian, R. J. "Direct numerical simulation of a $30 \mathrm{R}$ long turbulent pipe flow at $\mathrm{R}+=685$ : Large-and very large-scale motions". In: Journal of Fluid Mechanics 698 (2012), pp. 235-281. DOI: 10.1017/jfm.2012.81. 
[19] Hoyas, Sergio and Jiménez, Javier. "Scaling of the velocity fluctuations in turbulent channels up to $\operatorname{Re} \tau=2003$ ". In: Physics of Fluids 18.1 (2006), p. 011702. DOI: 10.1063/1.2162185.

[20] Wu, Xiaohua and Moin, Parviz. A direct numerical simulation study on the mean velocity characteristics in turbulent pipe flow. Vol. 608. 2008, pp. 81-112. DOI: $10.1017 /$ S0022112008002085.

[21] Issa, R. I. "Solution of the implicitly discretised fluid flow equations by operator-splitting". In: Journal of Computational Physics 62.1 (1986), pp. 40-65. DOI: 10.1016/0021-9991 (86)90099-9.

[22] Nicoud, F. and Ducros, F. "Subgrid-scale stress modelling based on the square of the velocity gradient tensor". In: Flow, Turbulence and Combustion 62.3 (1999), pp. 183-200. DOI: 10.1023/A:1009995426001.

[23] Jeong, Jinhee, Hussain, Fazle, Jinhee, Jeong, and Fazle, Hussain. "On the identification of a vortex". In: Journal of Fluid Mechanics 285.October (1995), pp. 69-94. DOI: doi:10.1017/S0022112095000462.

[24] Kolmogorov, A. N. "The Local Structure of Turbulence in Incompressible Viscous Fluid for Very Large Reynolds Numbers". In: Proceedings of the Royal Society A: Mathematical, Physical and Engineering Sciences 434.1890 (1991), pp. 9-13. DOI: 10.1098/rspa.1991.0075.

[25] Milgram, Jerome H. "Short wave damping in the simultaneous presence of a surface film and turbulence". In: Journal of Geophysical Research: Oceans 103.C8 (1998), pp. 15717-15727. DOI: 10.1029/98jc01191.

[26] Ling, Y., Fuster, D., Tryggvason, G., and Zaleski, S. "A two-phase mixing layer between parallel gas and liquid streams: Multiphase turbulence statistics and influence of interfacial instability". In: Journal of Fluid Mechanics 859 (2019), pp. 268-307. DOI: 10.1017/jfm. 2018. 825. 


\section{Chapter 4}

\section{Methodologies for simulations analysis of turbulence and droplets.}

\subsection{An overview of turbulence theory.}

The theory of turbulence is a quite complex matter, whose complete description goes far beyond the goal of this thesis. On the other hand, some concepts need to be introduced as they will be extensively used within this thesis. While an explanation of some basic concepts (such as the energy cascade and energy dissipation) will be addressed in the following subsections, it is important to understand why turbulence may be a fundamental tool for studying sprays and, in general, primary atomization.

A first important reason for studying turbulence in these type of problems, is to understand what are the typical length scales of the problem. While in Chapter 1 a brief discussion of the smallest scales of motion considered for spray was provided, an extensive description of how these scales can be calculated has not yet been provided (or at least exploited). In this thesis, an original methodology for achieving this goal has been developed based on a pseudo-fluid assumption.

Another reason for understanding the behavior of turbulence in sprays (especially the energy content at each scale) is given by the necessity of quantify- 
ing the interactions occurring between turbulent structures and liquid structures. As a matter of fact, there are some interesting researches in literature correlating the perturbations frequencies to the breakup $[1,2]$. Numerically, these perturbations can be estimated by computing the flow spectra and their energy content is a function of the Fourier coefficient at that frequency. Hence, these analysis may be an interesting way to relate breakup phenomena to turbulent structures if a comprehensive characterization of both processes is provided.

Finally, the study of turbulence and atomization developed in a rather similar fashion. While the first formal description of the turbulence nature was provided by Richardson [3], the first attempt of explaining how the energy is transmitted across scales was provided by Kolmogorov [4]. The idea behind this first discussions was that the turbulent velocity was self-similar, therefore the energy that was generated at larger scales was transmitted to smaller scales, until a dissipation range is reached. The whole process can be seen as a fractal one, where each scale transmits most of its energy towards smaller scales (not only) in order to reach a natural equilibrium, given by the energy dissipation through viscosity. As no geometrical fractal theory can be applied in this case, Kolmogorov [4] proposed that an equivalent log-normal process is occurring, and he calculated the exponent scaling typical of this process. On a similar path, Kolmogorov [5] discussed the breakup of carbon particles and proposed, again, the idea that a fractal process for the breaking process of each particle into a number of smaller particles. Following the assumption that each parent particle (of radius $r_{p}$ ) has a constant probability in breaking into a number of derivate particles (of radius $r_{d}$ ), the fractal representation of the process is give by $r_{d}=\alpha r_{p}$ where $\alpha$ is the fractal ratio. It comes as no surprise that the mathematical evolution of this equation leads to a log-normal representation of the breakup process. Gorokhovski and Herrmann [6] discussed how this fragmentation theory can be moved towards droplets breakup, justifying the usual log-normal droplet distribution observed in sprays.

It is evident that while the two processes, turbulence and atomization, are clearly different but they occur due to similar physical necessity. Turbulence is a natural response to an external force by the fluid, which is trying to dissipate the energy using viscous stress, hence transferring the energy to the scales in which such mechanism is possible. Viscosity in fact is not able to dissipate large amount of energy, hence the flow physically reacts by generating a gradient mechanism that allows for energy transport. Similarly, fragmentation and atomization are a natural response of the medium (solid in the former case and liquid in the latter) to external forces which destabilize the systems 
equilibrium. When a force is applied to the system, it deforms until a limit in its elasticity is reached. In order to absorb the whole energy, the system has to breakup and generate more surface that is able to absorb the excess energy. Lets simplify, for a moment, the breakup phenomena in atomizing liquids and assume that a initial large liquid structure of equivalent diameter $L_{l}$ is subject to an external energy $E$. The breakup process is equivalent to absorbing the amount of energy $\sigma \Delta A$ where $\Delta A$ is the amount of new area generated within the process. Assuming the liquid structure breaks into $n$ smaller structures, the volume conservation yields $L_{l, n}=L_{l} / n^{1 / 3}$. The new total surface will be $n L_{l, n}^{2}=n\left(L_{l} / n^{1 / 3}\right)^{2}=L_{l}^{2} n^{1 / 3}$ with the total amount of structures being roughly proportional to $n \approx\left(E / \sigma L_{l}^{2}\right)^{3}$. This mechanism is conceptually similar to the one occurring in turbulence, but the real key is the estimation of the energy $E$ and the scale on which occurs. In fact, in turbulent sprays, the energy available at each wavelength is promoting breakup and, intuitively, the more energy will be injected into the system, the more liquid surface will need to be created to absorb it.

In sprays, the main sources of energy generation are the injection momentum (from the high velocity liquid that outgoes the nozzle) and the KelvinHelmholtz instability, triggered by the shear stress produced between the relative velocity of the two phases. These sources generate both atomization and turbulence, creating an extremely complex scenario in which the two phenomena are actually interacting with each other. By following the KolmogorovHinze theory [7], the general belief is that droplets can be as small as the maximum stable droplet diameter allows $[7,8]$. Such a droplet diameter is the Hinze scale $\eta_{H}$. On the other hand, it has been proven in many works (e.g. [9]) that $\eta_{H}$ is over-estimating the droplet size, as smaller droplets are found to be formed in the mixing layer.

These reasons, highlight the needs of a solid methodology for analyzing turbulence and droplet properties in sprays. For this reason, the rest of the chapter will be devoted to provide a theoretical context first, and then a detailed explanation of the methodologies developed within this thesis to analyze the results of the DNS simulations. A special focus will be given to the energy cascade, as well as the calculation of the correlation function.

\subsubsection{Background on turbulence}

The main tools that will be used for analyzing the turbulence in this thesis are mainly the autocorrelation function and the energy cascade. Both derives from the early theories of Kolmogorov [4], that have later been slightly modified by including the studies of Wilson and Hulme [10]. As computing is just a late 
addition to the study of turbulence, most of the studies cited will be made using hot-wire anemometry. The discussion provided will only be partial and related to the topic used in the discussion. For a better understanding of the matter, the main books referenced in this discussion are Pope [11] and Cotton et al. [12].

The first thing that is at the bases of the turbulence theory used here are the hypothesis made by Kolmogorov [4], that will be presented in its original form just for sake of clarity, although the more complete formulation based on the structure functions [12] (generalized for the PDF in [11]) may be more useful for a deeper scientific insight. Nevertheless, a slight usage of these concepts will be done in order to improve the understanding of the methodology used in this thesis. For clarity, in the following discussion $u^{\prime}$ is the turbulent velocity component.

The famous statement by Richardson [3] is often used to summarize the first qualitative understanding that was available on turbulence:

"Big worls have little worls, which feed on their velocity;

And little whorls have lesser whorls, And so on to viscosity."

Unfortunately, the scientific community had to wait a couple of decades until the original discussion by Kolmogorov (in 1941) presented 3 principal hypothesis that built the foundations for the modern study of turbulence. Assuming that the reader has already previous understanding and knowledge of turbulence (and statistical tools), we can summarize the motion scales into $\mathcal{L}$, the characteristic flow lengthscale, $L$, the turbulent lengthscale (typical of larger eddies), and the smallest motion scale $\eta$. Figure 4.1 shows a schematic representation of the energy cascade process and where the scales are typically located. Consistently with [11], also the transition lengthscales between ranges $\ell_{E I}$ and $\ell_{D I}$ are represented. We can now present the hypothesis made by Kolmogorov in analyzing turbulent flows, integrated with some considerations from Cotton et al. [12]:

1. Local isotropy hypothesis: when Re is sufficiently high, the small scale motion is statistically isotropic. This first law is extremely important in analyzing turbulent flows and held to a more general formulation using structure functions [12]. Let's define a structure function as:

$$
\mathbf{S}_{n}(\mathbf{x}, \boldsymbol{\ell})=\left(\mathbf{u}^{\prime}(\mathbf{x}+\boldsymbol{\ell})-\mathbf{u}^{\prime}(\mathbf{x})\right)^{n} .
$$


The clear interpretation of the current hypothesis is that each component of $\mathbf{S}_{n}(\mathbf{x}, \boldsymbol{\ell})$ is invariant under any rotation of $\boldsymbol{\ell}$ and $\mathbf{S}_{n}$. The more general hypothesis of homogeneous at small scales can be then seen as invariance under any translation of $\mathbf{x}$, hence

$$
\mathbf{S}_{1}(\mathbf{x}, \ell)=\mathbf{S}_{1}(\mathbf{x}+\delta \mathbf{x}, \ell) .
$$

This actually corresponds to the $\mathbf{H 1}$ hypothesis in [12].

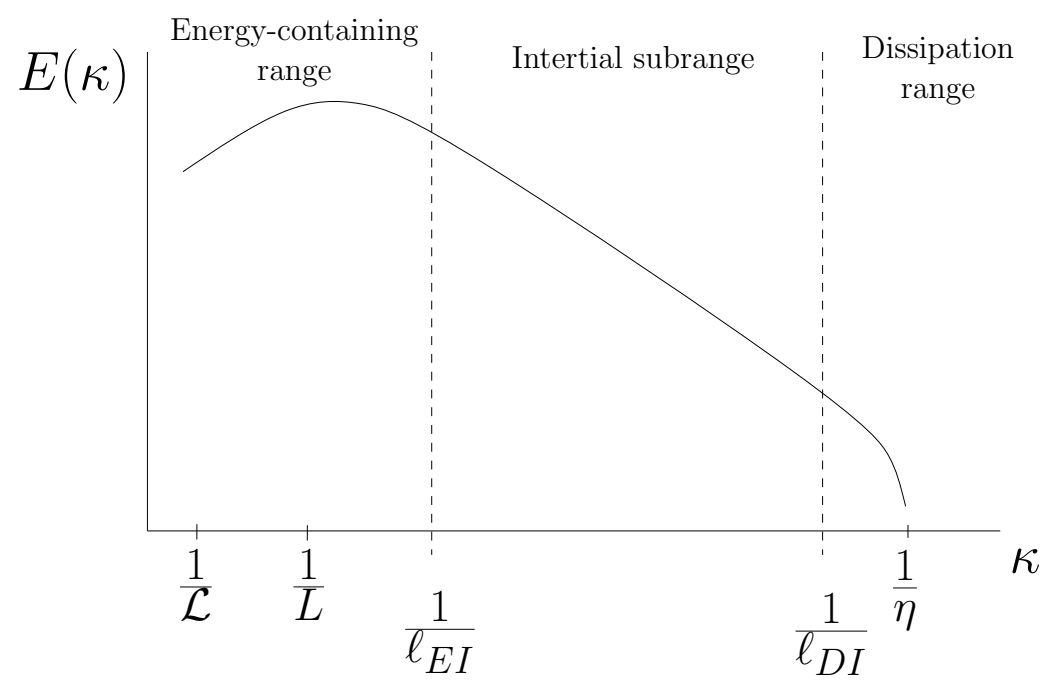

Figure 4.1: Schematic sketch of an energy cascade with the relevant lengthscales.

2. Kolmogorov's first similarity hypothesis: for a sufficiently high Re, at any scale smaller than the ones in the the energy-containing region, the flow small-scale statistics depends only by $\epsilon$ and $\nu$. This hypothesis, while widely used, poses a significant issue for multiphase flows. In fact, no clear definition of $\nu$ is possible in any location due to the atomization process. Furthermore, the usual definition of $\epsilon$ is:

$$
\epsilon=2 \nu\left\langle s_{i, j} s_{i, j}\right\rangle
$$

where $s_{i, j}=\left(\partial_{j} u_{i}^{\prime}+\partial_{i} u_{j}^{\prime}\right) / 2$ is the strain tensor of the fluctuating velocity. In many turbulence studies this is a absolute value. For example, in DNS of isotropic turbulence decay is often used a default value, while in most hot wire-anemometry just one point measurements are taken, hence $\epsilon$ becomes a 
scalar. In atomizing sprays, $\epsilon$ is indeed a function of space (it will be discussed later how this problem can be simplified). This definition in multiphase flows is affected by the uncertainties of having an undefined viscosity value at a given spatial location. Hence, this may lead to two different formulations of $\epsilon$ :

$$
\epsilon=2\left\langle\nu s_{i, j} s_{i, j}\right\rangle
$$

Equation (4.3) is a formulation widely used in literature for gas diffusion in turbulence (see the case of scalar field in [13]). This formulation becomes exact when the strain rate tensor is computed for very small $\delta x_{i}$, hence is usually accepted in DNS.

This issue is non-trivial and is one of the main factors driving researcher away from the usage of historical turbulence theories in multiphase flows. A discussion about some interesting results (achieved in the last 5-10 years) will be discussed later.

3. Kolmogorov's second similarity hypothesis: if the Reynolds number is sufficiently high, for all scales $\eta \ll \ell \ll L$, the motions is uniquely determined by $\epsilon$, hence independent from $\nu$. This is equivalent to say that the flow statistical behavior is uniquely a function of $\ell$ and $\epsilon$. This latest formulation, proposed by Cotton et al. [12], is useful in demonstrating the famous $-5 / 3$ law for turbulent spectrum. Using this statement with the second order structure function $S_{2}$ for $\ell$ in the prescribed range simply leads to $S_{2}=f(\ell, \epsilon)$, which dimensionally is obviously $[L]^{2}[T]^{-2}$. A simple dimensional analysis leads to the conclusion that

$$
S_{2}=C \epsilon^{2 / 3} \ell^{2 / 3}
$$

which is usually referred to as the $2 / 3$ law. Here, $C$ is a dimensionless constant as the discussion about its universality (or lack of) is currently ongoing, but not really of primary interest for this thesis.

To obtain the $-5 / 3$ law there are few ways (e.g. [11, 12]). Here we will used a slightly different approach compared to literature as it will clearly offer a deeper insight on why this theory needs to be valid in multiphase flows, where the above discussion about the definition of $\epsilon$ may raise doubts on this hypothesis applicability. Lets first define the two-point correlation function as:

$$
R_{i j}(\ell)=\left\langle u_{i}^{\prime}(x) u_{j}^{\prime}(x+\ell)\right\rangle .
$$


For the previous discussed properties of structure functions in homogeneous flows, Equation (4.5) for $i=j=1$ can be rewritten as:

$$
R_{11}(\ell)=\left\langle u^{\prime}(x)^{2}\right\rangle-\frac{1}{2} S_{2}(\ell) .
$$

The theory discussed by Wilson and Hulme [14] lead to the formulation of the one dimensional spectra, as:

$$
E_{11}\left(\kappa_{1}\right)=\frac{2}{\pi} \int_{0}^{\infty} R_{11}(\ell) \cos \left(\kappa_{1} \ell\right) d \ell .
$$

Substituting Equation (4.6) into Equation (4.7) and using a gammafunction $\Gamma$ for solving the convergent integral yield to:

$$
E_{11}\left(\kappa_{1}\right) \approx \frac{2 \Gamma(2 / 3) C}{\pi \sqrt{3}} \epsilon^{2 / 3} \kappa_{1}^{-5 / 3}=C_{2} \epsilon^{2 / 3} \kappa_{1}^{-5 / 3} .
$$

This law is extremely used in turbulence and usually is the one against which the turbulence data are presented both experimentally and numerically. It also gives us an interesting insight on the mathematical behavior of velocity in turbulent flow. If we discuss this in the context of Figure 4.1, this law can be demonstrated to be valid only in the inertial subrange. Here the velocity derivatives are non-smooth, as it can be mathematically proven for a function such as the one in Equation (4.8).

\section{Spectrum analysis in multiphase flows.}

In multiphase flows, the definition of $R_{11}$, which is only computed using the velocity field, is unchanged. Hence, the only assumption that has to be valid for a flow in order to verify Equation (4.8) is the statistical homogeneity. On the other hand, other spectrum are possible when one or more of the previous steps is not applicable to the case studied. For example, a widely famous case in multiphase flows is the so-called pseudo-turbulence. This regime is often observed in bubbly flows (see the studies of Mendez-Diaz et al. [15]) and light particles transport (e.g. Mercado et al. [16]). In this case, the power laws usually follows a -3 exponent. In those cases the energy transmission between scales is actually triggered by the shear caused by the bubbles rising in the flow. In these cases, it can be found a coexistence of $-5 / 3$ and -3 laws [17] and DNS studies [18-20] confirm that the lift produced by the bubbles (even when these are treated like particles) is a different mechanism in turbulence. In this 
case, it can often be seen a energy transmission from smaller scales toward larger scales. It should be noted that some authors [21] consider studies like the one in [18] to be not entirely representative due to a artificial forcing at low frequencies that deform the spectra and invalid the results.

On the other hand, these bubbly spectra studies show significantly different dynamics than what presented above. In these cases, in fact, the energy is not only produced in the energy-containing range hence the transmission process is completely different and cannot be generalized for all multiphase flows. In other words, in these cases the homogeneous statistic assumption does not hold. Despite that, these is a notable example of how source terms in the Navier-Stokes equations can create deviation in the energy spectra. An interesting review of bubbly flows can be found in [21], where the distinction of bubbles by their size (e.g. in respect to the Kolmogorov scale) is made.

Other notable studies on the deviation from a the ideal $-5 / 3$ behavior were performed bu Sundaram and Collins [22], based on the previous analysis of Eaton and Fessler [23] for particle laden flows. These authors described how the presence of particles increase the energy at the smaller scales while decreasing the energy at the larger scales (opposite behavior than bubbly flows). In particular, Sundaram and Collins [22] described the effects on the spectra if the velocity is correlated using a particle-particle, fluid-fluid or particle-fluid approach, demonstrating how the particle have a overall dissipative behavior, although far from the -3 exponent in pseudo-turbulence. An interesting review on this subject is provided in [24]. Other interesting studies, especially on particle/droplets of different size and in different condition are resumed in $[21]$.

\section{Spectra of multiphase sprays: literature review.}

A brief discussion of examples in literature of spectrum in sprays will be carried over in this section. As discussed previously, coupling multiphase flows and turbulence knowledge is often a difficult challenge and there is no theory nor guidelines for supporting these studies. Therefore, many authors have used spectrum in multiphase flows for the most different reasons.

One interesting consideration that can be made about spectrum, is that they provide informations on the total amount of energy resolved and can be used, in substitution of complete mesh convergence analysis for evaluating the amount of energy that is not resolved properly. For example, Duret et al. [25] studied the mixing of droplets and air in a periodic box with liquid structures at different average mass concentrations. The authors used, at 
first, the spatial spectra to check the mesh convergence between two cases of $128^{3}$ and $256^{3}$ elements, showing that their numerical methods (similar to the ones used in this thesis) do not affect large scales when mesh is not properly resolved. In other words, it is likely that the energy excess is either dissipated by the numerical scheme (which is obviously an unwanted situation) or so low that surface tension dumps it. Furthermore, Duret et al. [25] used a phase separated spectra approach for describing the liquid and gaseous spectra over a periodic domain. Interestingly, the author showed that a significant divergence from the theoretical spectra is reported while varying the amount of liquid percentage within the box. The results showed that, while larger scales are often displaying similar energy content, one of the two phases is absorbing part of the energy at lower scales, creating an interesting deviation of the spectra. On the other hand, the most interesting result showed in this work, is that different two-phase spectra do not behave in significantly different ways for different average liquid concentrations. Moreover, the author argued that this may be an effect given by the forcing term used to maintain a certain level of Turbulent Kinetic Energy (TKE). This result inspired some considerations that motivate the type of analysis used here, hence, a few comments for these approaches were used to formulate the new methodology:

- Using the two-phase spectra has actually few advantages. In a first place, it neglects the mass concentration, consistently with the definition of both autocorrelations and structure functions that are only velocitydependent.

- Two-phase spectra allow for comparison with single-phase flows. While many subgrid for LES models exists for modeling the energy dissipation in single-phase flows, no knowledge is universally accepted for multiphase flows. In most of the well-resolved LES simulation, the mesh is actually too coarse to capture atomization properly, hence the cell values are actually dependent of the mass concentration. In this sense, the LES dissipation models have no insight on separated contributions provided by the flows, which means that a two-phase spectra is probably more representative then a single-phase one. On the other hand, average turbulence properties should be properly estimated in order to allow for this simplifications.

While other works of the same group proposed similar analysis (e.g. [26]), the one presented is likely the most significant work they performed on the topic, discussing both the methodology and the results. 
Many other interesting observations have been made in literature. Ling et al. [9] used both the spatial and temporal spectra for analyzing a twodimensional coaxial spray. The spatial spectra was used to identify the inertial subrange (where the $-5 / 3$ law applies). They first analyze the instantaneous spectra and then elaborate on the time-average one. Although the influence of this work on the current thesis is limited (due to its publishing date), the approach they used actually corroborate the one used and developed here. In this case, they performed the analysis in the spanwise direction at different streamwise locations. The spanwise direction, similarly to the case of channel flows, is the direction where the turbulence presents a statistical stationary behavior, hence the anisotropy of the flow are not accounted for. A similar methodology has been developed in this thesis but for a more complex geometry. The authors also proposed the usage of a temporal spectra for analyzing the smoothness of the results and the correctness of the energy transmission rate (if any specific frequency was injecting energy into larger or smaller scales) and comparing it with dominant frequency analysis performed for the interfacial stability analysis.

Finally, [27] computed the spatial spectra in a planar jet using the streamwise direction. The spectra was used to demonstrate the turbulent behavior of the spray, but it is worth saying that while a streamwise spectra allows for more data on the longitudinal anisotropic structures of the spray, it is also likely increasing the energy generation range, hence creating more separation among scales.

All the studies presented show clearly how the spectra can be used for different purposes and the amount of information that can be derived out of it. On the other hand, atomizing sprays are significantly out-of-date when compared with state of the art single-phase flows, especially in terms of scales separation (e.g. estimate using $L / \eta$ ). The increment in computing time for capturing the breakup is one of the most significant reason for this discrepancy. Furthermore, a lack of a complete theoretical knowledge on turbulence in multiphase flows is also responsible. The estimation of the Kolmogorov scale in these type of flows is challenging due to the phase variations locally, the strong anisotropy of the flow and, again, the lack of literature references. This thesis tries to add on that, improving the knowledge on how to estimate the scales and how these results can be validated. 


\subsection{An overview of droplet analysis in DNS}

While a brief discussion on the terms choice was done in Chapter 1, we shall spend a few more words on the subject. The term granulometry per-se, is not used among atomization studies. The usual concept of droplet is usually connected to a spheric object [28] and many studies performing this type of studies have this as a fundamental feature characterizing droplets [9, 29]. A droplet in fact reaches the spheric shape when at rest from external perturbations (or at least the main ones). On the other hand, reducing this discussion to spheric droplets is a over-simplification of the breakup phenomena, hence in this thesis, every detached liquid structure is accounted for as a droplet.

Sprays simulations have actually a very complex scenario when it comes to breakup. In a first place, in the mixing layer, droplets are typically roughly characterized by more than 4 points per diameter, which are insufficient for allowing an accurate computation of surface propagation. Shinjo and Umemura [30] proposed in his series of works to neglect droplets smaller than 4 cellsper-diameters, tagging them as debris. On the other hand, these droplets may be the product of many events, such as well-resolved breakup, unresolved pinch-off, satellite atomization among the most important ones. The criteria used actually varies from case to case, depending on the goal of the analysis. The usage of a step-by-step Lagrangian methods for detection [28, 31] is inherently time consuming and reduced to just spherical droplets. The most accurate procedure for analyzing all the liquid structures is analyze the simulation snapshots. On the other hand, the time-output resolution needs usually to be quite coarse due to the storage space required for each snapshot in petascale simulations (with several billions of cells [9, 32]). Again, using on the fly statistics may be excessively time-consuming in the simulation and does not allow to check the method sensitivity. All this aspects, will be discussed further but are usually not addressed in sprays simulations.

\section{Spray droplet analysis in literature.}

Many analysis on spray droplets have been performed in literature and most of these analysis are focused on droplet-size distributions.

Shinjo and Umemura [30] performed an analysis on droplet-size distribution on a transient spray, showing the effect of the velocity on the resulting histograms. These analysis highlight how the higher the Reynolds number, the more the atomization is displayed (intended as higher number of droplets) and the smaller the droplets get. On the other hand, transient results are 
actually only indicative of the atomization generated by the spray tip, hence not really relevant for statistical stationary studies.

"On simulating primary atomization using the refined level set grid method" [33] used a decoupled grid between velocity and Level-Set to evaluate the mesh dependency for the droplet distribution of size (intended as diameter), volume and surface area. The author argued that convergence can be achieved on the droplet-size distribution by refining the mesh, although many authors argues otherwise [27].

Desjardins et al. [34] used PDF for both velocity and droplet-size distributions in order to validate his code results for a coaxial spray against PIV results. The results show that the overall behavior is well captured but a significant offset is observed.

Ling et al. [29] performed a study on the influence of the mesh size on atomization. The real aim of the analysis is actually the comparison of gamma and log-normal distribution models compared against well resolved simulations data. The results indicate that the more the mesh is refined, the more a log-normal distribution is capable of describing the PDF of the droplet-size distribution. Furthermore it also shows an interesting fact, as no simulation actually seems to reach a mesh convergence, even for a case that has proven to resolve the Kolmogorov scale, according to the author estimation in [9]. This work is a perfect example of the type of results that are currently being pursued by the scientific community. The main issue, is the the comparability against experimental data, which are extremely rare and performed in conditions that are difficult to reproduce due to high Reynolds number. Another often limiting aspect of reproducing experiments numerically is that optical techniques, such as PIV that are capable of gathering data on droplet size distribution, need to be applied in the far field of the spray, which requires numerically to solve a huge domain.

An interesting approach, that inspired the analysis performed in this thesis, is proposed in the work of Subramaniam [35, 36]. This studies suggests to use point-process theory, in combination with notions from statistical mechanics to characterize the droplet distribution and properties. While in a first instance, this approach was conceived for initialing simulation with physically reliable conditions, it may be used to extract physical knowledge from the droplet distributions. The author made a complete and detailed description of the two main approach that can be used, the Klimontovich and the Louisville description of the droplet [35]. In the first case, droplets are seen as described by a probabilistic function in a 8-dimension space, in $(\mathbf{u}, \mathbf{x}, d, t)$ where $d$ is the droplet diameter. This statistical description of the droplets is the one used 
in that inspired part of the analysis performed. The resulting fine-grained density function can be written as:

$$
f(\mathbf{x}, \mathbf{u}, d, t)=\sum_{i=1}^{N_{d}(t)} \delta\left(\mathbf{x}-\mathbf{X}^{(i)}(t)\right) \delta\left(\mathbf{u}-\mathbf{U}^{(i)}(t)\right) \times \delta\left(d-D^{(i)}(t)\right) .
$$

The droplet time-dependence is used if evaporation is considered, but in that case also the temperature value should be added. Overall, the temporal dependence can be omitted in case of temporal average, which is the approach used in this thesis. By defined integration in one or more dimensions, one can obtain the number of droplets that satisfy the requirements imposed by the integration range.

The mere droplet distribution function is partially unable to provide full informations on how the droplets are generated, where and when, while the Klimontovich approach is. It is the author personal opinion that a mathematical interpretation can be found in such a way that the why can also be answered. That would open a new way of studying DNS of atomizing sprays.

Understanding the causality of phenomena that lead to the droplet generation is definitely a non-trivial task. Such a knowledge is usually provided by one-dimensional stability theory and analysis of the surface instability generated by both the physical properties of the system, as well as the turbulence field developed. But an approach, such as the one proposed in Equation (4.9) may be an interesting way to propose a useful simplification of the problem, while still allowing to change its dependencies (e.g. adding turbulence variables) that may be helpful in developing subgrid models for RANS and LES simulations.

\subsection{Methodology for turbulence analysis in DNS}

In a first place, the two most important scales in the study of turbulence are Kolmogorov time $\left(\tau_{\eta}\right)$ and space $(\eta)$ scale:

$$
\begin{aligned}
& \eta=\left(\frac{\nu^{3}}{\epsilon}\right)^{1 / 4} \\
& \tau_{\eta}=\left(\frac{\nu}{\epsilon}\right)^{1 / 2}
\end{aligned}
$$


Where $\epsilon$ is the energy dissipation rate. Furthermore, in the present work, statements like to "turbulence level" or "higher turbulence intensity" are referring to the Taylor-Reynolds number, defined as:

$$
R e_{\lambda}=\frac{\sqrt{\left\langle u^{\prime 2}\right\rangle} \lambda}{\nu}
$$

where $\left\langle u^{\prime 2}\right\rangle$ is referring to the average of the summed square of each velocity component and $\lambda$ is defined as the Taylor microscale from [14]:

$$
\lambda=\sqrt{\frac{15 \nu\left\langle u^{\prime 2}\right\rangle}{\epsilon}}
$$

As by definition, $\eta$ and $\tau_{\eta}$ are the smallest scales occurring in a turbulent flow, it seems natural to take them as the resolution to use in the analysis of the flow (obviously, the first spatial and the latest temporal). On the other hand, despite many correlation have been used to estimate a-priori these scales [11], none of them can be defined as conclusive in absence of a complete estimation of the energy dissipation rate. Although these approaches are useful ways to approximately determine the order of magnitude of these scales they are poor indicator of the scales to use in the analysis of these turbulent flows. It is therefore intuitive that the scales that should be considered for the analysis are the cell size $\Delta$ and the time-step $t_{s}$ used in the simulation. In other words, the sampling of the simulation results should be output each cell value, at each time-step.

It is obvious that the analysis of these scales can generate a conspicuous amount of data, hard to process even on the most advanced super computers. Therefore it is important to select which data to extract and how to process them. An appropriate starting point for determine which portion of the domain is most suitable to study sprays, would be the analysis of statistical field, such as average and standard deviation of density, velocity and pressure along the whole domain. Unfortunately, even with the most recent DNS simulations, no real statistical study has been conducted and nor analysis of the flow spectra, which is one of the main aim of this work.

On the other hand, the lack of data on sprays (both numerical and experimental), may be compensated by using data from single-phase jets, which are extensively documented since some are among of the very first analysis of energy spectra computed [37] and have been continuously analyzed and improved over the years [38-41]. The analogy between multiphase flow sprays and jets is quite straight forward. Both fluids are injected in a calm and quiescent 
environment after passing though a cylindrical nozzle where the flow is turbulent (therefore have "similar" dimensionless velocity profiles). Both flows have a main velocity component, namely the axial. Both have a Gaussian velocity decay along the radial coordinate after the Near-Field is surpassed (therefore approximately when $x>25 D$ ), which is generated by shear with the calm quiescent air in the domain and is a function of the axial position; such a decay determines the velocity angle, widely characterized both experimentally and numerically in jets and sprays. An obvious consequence of the latest similarity is that the total momentum need to be conserved while the axial velocity decays, therefore more momentum is transmitted to the points which are at a higher radial distance progressively, until the velocity eventually matches the one of the calm air (and in other words reaches zero). As the velocity decreases radially, it may be assumed that the turbulence intensity decreases accordingly after the mixing layer. These considerations lead to the assumption that at a fixed axial distance, on each iso-radius curve (for each point located at the same radial distance from the jet centerline), the flow statistics need to be statically stationary. In other words, if we use the cylindrical coordinates $(x, r, \theta)$, fixing the first two coordinates at $x=x_{p}$ and $r=r_{p},\left\langle u_{i}(\theta)\right\rangle=$ const. and $\left\langle u_{i}^{\prime 2}(\theta)\right\rangle=$ const.. A representation of such a behavior is showed in Figure 4.2, where the iso-radius lines are displayed (in blue) in relation to the flow average velocity at $x / D=25$. The function $u^{\prime}(\theta)$ at each time-step will obviously be a periodic function of period $2 \pi$ and can be adequately defined with $\theta \in(-\pi, \pi]$.

By being $u^{\prime}(\theta)$ representative of a specific turbulence level, this represents a set of data easy to compare to Hot-Wire Anemometry (HWA) studies of jets. In fact, the usual way to operate in this technique [40] is to collect first a set of time-variant data using a hot-wire sensor, then convert it to space-variant using the Taylor hypothesis:

$$
\partial_{x} u=\frac{1}{u} \partial_{t} u
$$

which is commonly known as the Taylor frozen-turbulence hypothesis [10]. While this hypothesis has not been definitively proven to be valid in freeshear flows [42], it has the inherited advantage of referring to just one point, hence only one level of turbulence, consistent through the whole period of the statistic averaging. In fact, the way in which a monodimensional spectra of the energy is usually calculated in spray is directly applying a Fast Fourier Transform to the axial velocity. As this method is supposed to capture the energy content associated with the eddies, as the turbulence intensity increases along 

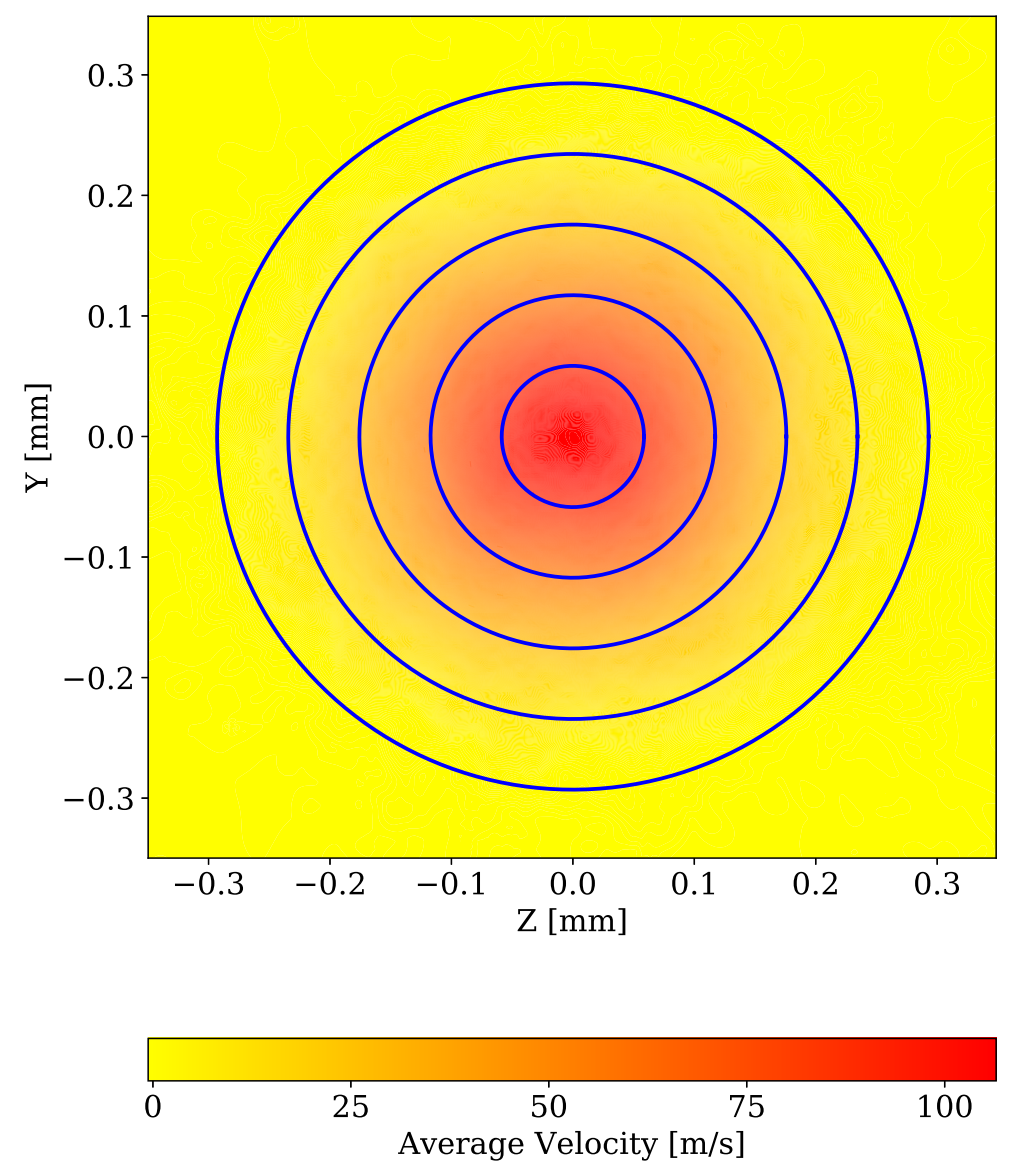

Figure 4.2: Time-average axial velocity field over the analysis plane at $x / D=$ 25 for the $M B C$ case (that will be discussed in more details later on). The lines represents the iso-radius curves over which the flow presents a statistical stationary behavior. 
the centerline [43], this method may lead to overestimation or underestimation of energy at certain frequencies.

Following up on these considerations, the usage of $u(\theta)$ is safely allowing to evaluate all turbulent structures along the azimuthal direction while providing a complete direction even for sprays/jets in which no complete penetration is reached.

All the considerations done lead to the conclusion that a sub-domain reaching the whole radial extension of the spray should be used. In other words, if $x$ is the spray penetrating direction, the whole extension over $y, z$ should be extracted at each time-step, while on the $x$ direction only 3 points can be extracted, which are required to calculate $\partial_{x} u_{i}$ in the strain tensor and should be sufficient if the mesh size is small enough. This will allow to compute with accuracy a fist order derivative scheme, but may be noisy and insufficient for computing energy balances.

On the other hand, the axial position for the area to extract, hereafter called $\Omega$, still remains to be assessed. In order to fix that, the study of the influence of the $L$ parameter over the DNS simulation was used. Here it is found that once a turbulent inlet boundary condition is used, a spray axial breakup is reached and the spray intact core length is no more visible after $x / D>10$. At this point, it is important to remember that part of these methodology is based on the assumption that an analogy between a multiphase and a singlephase flow can be used to study the behavior of the turbulence in sprays. This assumption leads to the implicit consequence that a smooth field for all the fluid properties should be defined as we try to solve Equation (4.10). For this reason, it is important to locate $\Omega$ in a position in which no sharp interphase can be detected stably during the whole simulation. Furthermore, a more diluted region seems more indicated for such an analysis as it is statistically impossible to have any point with a probability of 1 of having liquid during the whole averaging period. Of course, if any droplet that loses momentum may stagnate in the domain, these points are usually less turbulent, therefore out of interest for the present analysis. These considerations positioning $\Omega$ at $x / D \approx 25$.

Once $\Omega$ has been positioned, the first step is the calculation of statistics, most notably the average fields. One-point statistics are most notably $\langle U\rangle$., $\left\langle u^{\prime 2}\right\rangle$ and $\langle C\rangle$ (where \langle\rangle indicates the temporal average). The last one in particular, allows the usage of Equation (2.2) for computing the average fluid properties. Let's now define the field $P$ as:

$$
P(x, y, z)=\langle C\rangle
$$


which could be defined as the probability of finding liquid at any given moment in a specific location of the simulation. Obviously, the value of $P$ varies continuously between 1 (liquid phase) and 0 (gas phase). It could be observed that, for VOF simulation, the field $P$ could be seen as a time average of the color-function $C$, but in order to maintain a general approach it is useful to rethink of it in terms of probability. The extension of this method to other simulation methods or to experimental analysis is still under investigation and it will be object of future analysis. Equation (2.2) can be therefore easily adapted to compute the average density and viscosity in time.

A few words should be spent in describing the implications of this method. Contrary to more fundamental studies, in sprays the prediction of the local average properties is non- trivial. In the study of isotropic turbulence with multiphase flows [25] the gas-liquid proportion is actually an input. While there are some studies discussed above about the transport and diffusion of gaseous species using turbulent jets, the break-up process increases the complexity of the study. While using the variable $P$ may seem a trivial solution, it actually hides a few important considerations. In a first place, this method requires smooth statistics, which may require a larger ensemble time than velocity statistics (first order). Furthermore, even with infinite simulation time, the average may be non-smooth. As it will be discussed later, there will be droplets that reach the far radial position in the domain, losing most of their kinetic energy. This means that, if the droplets stops in some point in space, this point will have a average value of $P$ equal to 1 . This issue can be avoided if a previous knowledge of the system is available. In our case, we already discussed that $u^{\prime}(\theta)$ is a statistically stationary direction. This assumption may be extended as well to $P$, as there are no reason for it not to be applicable to all fields. If this assumption is correct, both a smooth value of $P$ and velocity statistics can be achieved, therefore this hypothesis will be later proven to be verified in the simulations performed in this thesis.

The usage of the parameter $P$, is quite similar to the pseudo-fluid approach, used in various simulations, both for RANS/LES atomization simulations [44] and in chemical environments, e.g. particle flows [45]. In the first case, this approach is used so that the turbulence models can be used assuming that a sufficient mixing occurs in way smaller scales than the ones computed. This hypothesis is almost always correct, but the effects of applying the method are never verified. In the solid-liquid cases, some of the small size particle are assumed to be way smaller than the large particles and therefore their properties are accounted for in the liquid ones, creating a pseudo-fluid. Here this term, although correct, means that although the fluid that occupies a cell changes continuously during the simulation, its temporal 
average is used. The real meaning of that is that dissipation, and therefore viscosity, are actually behaving as a superposition of the effects generated by the two phases. Therefore, the assumption is that:

$$
\epsilon=2\left\langle\nu s_{i, j} s_{i, j}\right\rangle \approx 2\langle\nu\rangle\left\langle s_{i, j} s_{i, j}\right\rangle
$$

which will be discussed in the results.

\section{Methodology for the Spectral Analysis}

There are several ways of obtaining a monodimensional spectra for a specific fluctuating velocity component. In the present work, the method by [10] is used to calculate the autocorrelation first, and then the flow energy spectra.

The autocorrelation function is generally written as:

$$
R_{i j}(\delta x, t)=\left\langle u_{i}^{\prime}(x+\delta x, t) u_{j}^{\prime}(x, t)\right\rangle
$$

Where $\delta x$ represents the lag parameter used as distances between the two correlated points in space. In the studied case, the autocorrelation could be computed over the whole range of the azimuthal coordinate $\theta$, which is the direction over which a statistical stationary behavior can be observed. Still, as $u(\theta)$ is a periodic function, the autocorrelation should not tend to 0 asymptotically, instead should reach a peak again at $\theta=2 \pi$, as the signal returns close to its original value. For this reason Equation (4.16) can be rewritten in its discretized form (for a single time step):

$$
R_{i j}(\xi)=\sum_{n=0}^{N_{\theta}-1} u_{i}^{\prime}(d+\Delta \theta) u_{j}^{\prime}(\Delta \theta)
$$

where $N_{\theta}$ is the number of element of the $\theta$ vector and $\xi$ is the discrete distance vector (that goes linearly from 0 to $\Delta \theta\left(N_{\theta}-1\right)$. By being the signal periodic, any point has $N_{\theta}-1$ points on which $R_{i j}(\xi)$ can be computed, per each value of $d$. Therefore, a useful way to visualize the process is concatenate twice the signal $u_{i}^{\prime}(\theta)$, but only compute $R_{i j}(\beta)$ until the point $N_{\theta}-1$. Finally, any spurious frequency can be filtered by calculating the average $R_{i j}(\beta)$ over the total number of time-steps.

The non-dimensionless autocorrelation function can be used to calculate the one dimensional spectra:

$$
E_{i} j\left(\kappa_{1}\right)=\frac{1}{\pi} \int_{0}^{\infty} R_{i j}(\xi) e^{-i \kappa_{1} \xi} d \xi
$$




\subsection{Methodology for droplet analysis in DNS}

Tagging has been widely used in multiphase flows in general for bubbles and droplets. The application of this methodology to Lagrangian Point-Particle (LPP) is a clear example [31] and excellent methodologies have been developed and employed [28] for achieving this goal. On the other hand, due to the implicit necessity of having a spheric droplet for the drag model added as a source term in Equation (2.1b) only spherical liquid structures are detected. Furthermore, this methodology is often used as a debris removal algorithm to improve the code stability [30]. In general terms, in this work the whole domain is scanned for any liquid structure of any shape and velocity, that are afterwards reduced into a sphere of equivalent mass, center of mass and mean velocity to the original liquid structure. This method, while neglecting many features of the fluid structures is inherently simpler to handle. In fact, it offers a practical and easy way to compare directly the results from DNS simulations to Discrete Droplet Method (DDM) [46]. While many mechanisms are used in RANS and LES simulations in order to model the breakup (from bigger to smaller droplets) this way of analyzing the DNS results may open the way towards a more insightful understanding of the breakup dynamic and a more comprehensive model.

The post-process routine relies on several sequential steps. On the other hand, the processing is performed in parallel using distributed memory (MPI). Similarly to ParisSimulator, the code splits the whole domain in $N$ subdomains. The number of processors per side $\left(n_{i}\right.$ where i can be $x, y$ or $z$ ) is previously decided so that the number of cells per side is perfectly divisible by the number of cores per side. Each cell has an absolute index for uniquely identification that will be used in case liquid structures that are on the boundaries between subdomains can be recognized and later joined. Furthermore, a threshold value for the color function, $C_{t h}$, should be previously determined. The steps that each processor performs are:

1. Remove the spray intact core: in this step all the cells that are continuously attached to the nozzle outlet are removed by the computational domain. The removal procedure starts from each cell in the injection section and checks if at each successive cell in the $\hat{x}$ direction has $C \geq C_{t h}$ (any cell verifying this condition is hereafter considered as full, otherwise empty). If so, the cell index is added to the collection of 
indexes composing the spray core and the cell color function and velocity are saved as well aside the index. The cell is then emptied, hence $C=0$. Finally a recursive algorithm is used to check if each cell is attached to other full cells and recursively so until only empty cells remains in the nearby. This last iteration helps to detect all the ligaments attached to the spray core and remove them as well. Each process performs this function on his own sub-domain and they are ultimately joined using the cells indexes.

2. Detect the droplets: each cell of the domain is scanned to check whether or not is full. If so, the recursive scanning of the cells nearby is once again called. If the code runs into a boundary or into a limit of recursive depth the liquid structure is marked for merging (which will be done at the end). Per each cell composing the droplet, only the index and the values for $C$ and $\mathbf{u}$ are stored and are uniquely attributed to that droplet.

3. Droplet merging from processes: each process results are collected and marked cells are checked for merging. If such an event happens, the cells attribute are merged.

4. Droplet properties calculation: The droplet volumetric diameter is computed as $d_{v}=\sqrt[3]{6 V_{d} / \pi}$ where the droplet volume $V_{d}$ is computed using the cell color function and the cell volume. The velocity and the center of mass are computed as a weighted average of the velocity components and the coordinates.

The results of the procedure described above is showed in Figure 4.3, where an exemplifying snapshot for the MBC case (that will be explained and discussed later) is shown.

The data displayed in the following chapters show the transient as well as the statistically stationary behavior. For the transient, each snapshot is analyzed every $0.5 \mu s$ until the transient time, $T_{t}$, is achieved. The droplet number, divided per diameter will be represented against time. For the statistically stationary behavior, a snapshot is analyzed every $10 \mu s$ from $T_{t}$ until $T_{t}+T_{s s}$, where $T_{s s}$ is the duration of the statistical stationary phase of the simulation. All the snapshots resulting datasets are averaged over $T_{s s}$. The reason for the difference in the time sampling lays within the different goals set for each analysis. The transient analysis, in fact, relies on high frequency snapshot to try capturing the general dynamics that dictate the spray formation and the droplet generation. On the other hand, as it will be showed more 


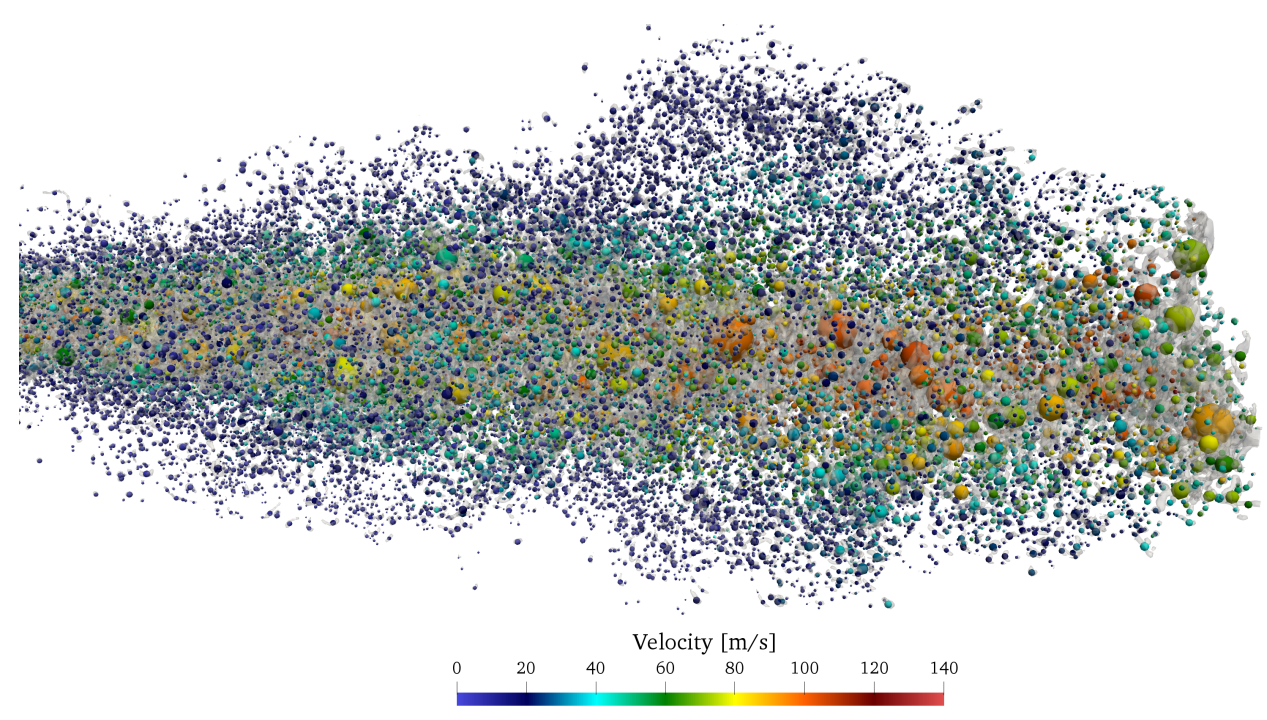

Figure 4.3: Results of the droplet detection procedure at $t=T_{t} / 4$ with $C_{t h}=$ 0.4

clearly in the following sections (and already observed in [32, 47]) most of the smallest droplets that are located at the spray cone periphery have already lost significant kinetic energy due to the air drag. Furthermore, most of the larger droplets (hereafter, every liquid structure will be called droplet regardless of its radius) are generated by the spray breakup and are advected by the main flow. In order to allow a significant time for some of these droplet to leave the domain (if such an event will occur), a significant time interval between each snapshot is required and a longer time for statistics as well. These statements will be supported by analysis later.

As explained above, the only parameter that can be picked in the algorithm is $C_{t h}$. For this reason, it is worth understanding the implication of the selected value and how, even if just in a qualitative way, this parameterization for the value $C_{t h}$ may help in understanding certain phenomena.

Figure 4.4 shows the the effect of the value $C_{t h}$ during the granulometric analysis in the statistically stationary part of the simulation. A first significant trend can be noted in the smallest range of droplets, e.g. between $d_{v} \in[2.34,3.80[$. This range represents droplet of the diameter close to the simulation mesh size $d x$. While these droplets are clearly meaningless physically and indicate, at best, an under-resolution of the mesh size for this type of simulation, they underline a specific behavior of the post-processing algorithm. 


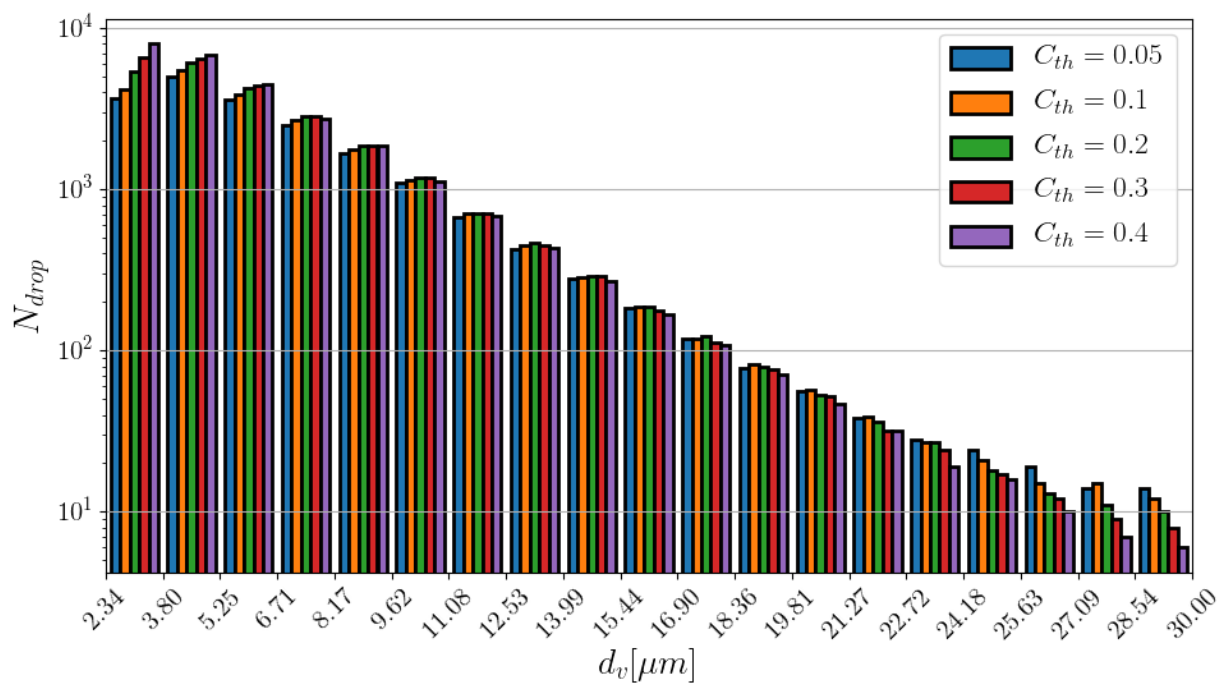

Figure 4.4: Parameterization of the $C_{t h}$ parameter during the statistically stationary part of the simulation (from $T_{t}$ until $T_{t}+T_{s s}$ )

In fact, the lower $C_{t h}$ is, the lower the number of droplets detected $N_{d r o p}$, which shows that indeed in the simulation many liquid structures are hold together by very thin liquid ligaments, which are represented by low $C$ single cells connected with the droplet. Therefore, the higher $C_{t h}$, the more this liquid structures will be split into two (or more) droplets. This lead to a obvious underestimation of larger droplets for high values of $C_{t h}$. In other words, low values of $C_{t h}$ accounts for ligaments on the verge of atomizing (where the low value of $C$ compose the ligaments) as being part of larger structures, hence increasing the number of larger droplets. On the other hand, this ligaments are likely to be breaking up after few simulation timesteps, hence accounting for them as a small droplet is not strongly misleading when analyzing the results.

More importantly, Figure 4.4 highlights how there is a certain number of ranges, where $d_{v} \in\left[6.71,22.72\left[\right.\right.$, for which $N_{\text {drop }}$ is quite close, regardless of the value picked for $C_{t h}$. In the authors opinion, this is actually the region where the analysis should be focused and where the most reliable data are provided. 


\section{References}

[1] Vassallo, P. and Ashgriz, N. "Satellite Formation and Merging in Liquid Jet Breakup". In: Proceedings of the Royal Society A: Mathematical, Physical and Engineering Sciences 433.1888 (2006), pp. 269-286. DOI: 10.1098/rspa. 1991.0047.

[2] Ashgriz, Nasser. Handbook of Atomization and Sprays. Springer Science \& Business Media, 2011. DOI: 10.1007/978-1-4419-7264-4.

[3] Richardson, Lewis F. "Weather prediction by numerical process, second edition". In: Weather Prediction by Numerical Process, Second Edition (1922), pp. 1-236. DOI: 10.1017/CB09780511618291.

[4] Kolmogorov, A. N. "The Local Structure of Turbulence in Incompressible Viscous Fluid for Very Large Reynolds Numbers". In: Proceedings of the Royal Society A: Mathematical, Physical and Engineering Sciences 434.1890 (1991), pp. 9-13. DOI: 10.1098/rspa.1991.0075.

[5] Kolmogorov, A. "On the log-normal distribution of particles sizes during breakup process". In: Dokl. Akad. Nauk. XXXI.4 (1941), pp. 99101.

[6] Gorokhovski, Mikhael and Herrmann, Marcus. "Modeling Primary Atomization". In: Annual Review of Fluid Mechanics 40.1 (2008), pp. 343366. DOI: 10.1146/annurev.fluid.40.111406.102200.

[7] Lasheras, J. C., Eastwood, C., Martínez-Bazán, C., and Montaes, J. L. "A review of statistical models for the break-up an immiscible fluid immersed into a fully developed turbulent flow". In: International Journal of Multiphase Flow 28.2 (2002), pp. 247-278. DOI: 10.1016/S03019322(01)00046-5.

[8] Eastwood, C. D., Armi, L., and Lasheras, J. C. "The breakup of immiscible fluids in turbulent flows". In: Journal of Fluid Mechanics 502 (2004), pp. 309-333. DOI: 10.1017/S0022112003007730.

[9] Ling, Y., Fuster, D., Tryggvason, G., and Zaleski, S. "A two-phase mixing layer between parallel gas and liquid streams: Multiphase turbulence statistics and influence of interfacial instability". In: Journal of Fluid Mechanics 859 (2019), pp. 268-307. DOI: 10.1017/jfm. 2018. 825. 
[10] Wilson, S. D. R. and Hulme, A. "The Effect of Bubbles Attached to an Electrode on Electrical Resistance and Dissolved Gas Concentration". In: Proceedings of the Royal Society A: Mathematical, Physical and Engineering Sciences 387.1792 (1983), pp. 133-146. DOI: 10.1098 / rspa. 1983.0054.

[11] Pope, Stephen B. Turbulent Flows. 2001. DOI: 10.1088/0957-0233/ 12/11/705.

[12] Cotton, William R., Bryan, George, and Heever, Susan C. van den. Turbulence. Vol. 99. C. Cambridge University Press, 2011, pp. 53-86. DOI: $10.1016 /$ S0074-6142(10)09909-2.

[13] Gauding, M., Danaila, L., and Varea, E. "One-point and two-point statistics of homogeneous isotropic decaying turbulence with variable viscosity". In: International Journal of Heat and Fluid Flow 72.May (2018), pp. 143-150. DOI: 10.1016/j . ijheatfluidflow . 2018.05. 013.

[14] Wilson, S. D. R. and Hulme, A. "The Effect of Bubbles Attached to an Electrode on Electrical Resistance and Dissolved Gas Concentration". In: Proceedings of the Royal Society A: Mathematical, Physical and Engineering Sciences 387.1792 (1983), pp. 133-146. DOI: $10.1098 /$ rspa. 1983.0054.

[15] Mendez-Diaz, S., Serrano-García, J. C., Zenit, R., and HernándezCordero, J. A. "Power spectral distributions of pseudo-turbulent bubbly flows". In: Physics of Fluids 25.4 (2013). DOI: 10.1063/1.4800782.

[16] Mercado, Julián Martínez, Prakash, Vivek N., Tagawa, Yoshiyuki, Sun, Chao, and Lohse, Detlef. "Lagrangian statistics of light particles in turbulence". In: Physics of Fluids 24.5 (2012). DOI: 10.1063/1.4719148.

[17] Prakash, Vivek N. et al. "Energy spectra in turbulent bubbly flows". In: Journal of Fluid Mechanics 791 (2016), pp. 174-190. DOI: 10.1017/ jfm. 2016.49 .

[18] Mazzitelli, Irene M., Lohse, Detlef, and Toschi, Federico. "On the relevance of the lift force in bubbly turbulence". In: Journal of Fluid Mechanics 488.488 (2003), pp. 283-313. DOI: 10 . 1017 / S0022112003004877.

[19] Mazzitelli, Irene M. and Lohse, Detlef. "Evolution of energy in flow driven by rising bubbles". In: Physical Review E - Statistical, Nonlinear, and Soft Matter Physics 79.6 (2009), pp. 1-9. DOI: $10.1103 /$ PhysRevE.79.066317. 
[20] Roghair, Ivo et al. "Energy spectra and bubble velocity distributions in pseudo-turbulence: Numerical simulations vs. experiments". In: International Journal of Multiphase Flow 37.9 (2011), pp. 1093-1098. DOI: 10.1016/j.ijmultiphaseflow. 2011.07.004.

[21] Elghobashi, Said. "Direct Numerical Simulation of Turbulent Flows Laden with Droplets or Bubbles". In: Annual Review of Fluid Mechanics 51.1 (2018), pp. 217-244. DOI: 10.1146/annurev-fluid-010518040401.

[22] Sundaram, Shivshankar and Collins, Lance R. "A numerical study of the modulation of isotropic turbulence by suspended particles". In: Journal of Fluid Mechanics 379 (1999), pp. 105-143. DOI: 10.1017/ S0022112098003073.

[23] Eaton, J. K. and Fessler, J. R. "Preferential concentration of particles by turbulence". In: International Journal of Multiphase Flow 20.SUPPL. 1 (1994), pp. 169-209. DOI: 10 . 1016/0301-9322(94) 90072-8.

[24] Toschi, Federico and Bodenschatz, Eberhard. "Lagrangian Properties of Particles in Turbulence". In: Annual Review of Fluid Mechanics 41.1 (2009), pp. 375-404. DOI: 10.1146/annurev.fluid.010908.165210.

[25] Duret, B. et al. "DNS analysis of turbulent mixing in two-phase flows". In: International Journal of Multiphase Flow 40 (2012), pp. 93-105. DOI: 10.1016/j.ijmultiphaseflow.2011.11.014.

[26] Chesnel, J., Menard, Thibaut, Reveillon, Julien, and Demoulin, Francois-Xavier. "Subgrid analysis of liquid jet atomization". In: Atomization and Sprays 21.1 (2011), pp. 41-67. DOI: 10.1615/atomizspr. v21.i1.40.

[27] Pitsch, H. and Desjardins, Olivier. "Detailed Numerical Investigation of Turbulent Atomization of Liquid Jets". In: Atomization and Sprays 20.4 (2010), pp. 311-336. DOI: 10.1615/atomizspr.v20.i4. 40.

[28] Herrmann, M. "A parallel Eulerian interface tracking/Lagrangian point particle multi-scale coupling procedure". In: Journal of Computational Physics 229.3 (2010), pp. 745-759. DOI: 10.1016/j.jcp.2009.10.009.

[29] Ling, Yue, Fuster, Daniel, Zaleski, Stéphane, and Tryggvason, Grétar. "Spray formation in a quasiplanar gas-liquid mixing layer at moderate density ratios: A numerical closeup". In: Physical Review Fluids 2.1 (2017), p. 014005. DOI: 10.1103/PhysRevFluids.2.014005. 
[30] Shinjo, J. and Umemura, Akira. "Simulation of liquid jet primary breakup: Dynamics of ligament and droplet formation". In: International Journal of Multiphase Flow 36.7 (2010), pp. 513-532. DOI: 10 . 1016/j.ijmultiphaseflow . 2010.03.008.

[31] Ling, Y., Zaleski, S., and Scardovelli, R. "Multiscale simulation of atomization with small droplets represented by a Lagrangian pointparticle model". In: International Journal of Multiphase Flow 76 (2015), pp. 122-143. DOI: 10.1016/j.ijmultiphaseflow.2015.07. 002.

[32] Shinjo, J. and Umemura, A. "Detailed simulation of primary atomization mechanisms in Diesel jet sprays (isolated identification of liquid jet tip effects)". In: Proceedings of the Combustion Institute 33.2 (2011), pp. 2089-2097. DOI: 10.1016/j.proci.2010.07.006.

[33] "On simulating primary atomization using the refined level set grid method". In: Atomization and Sprays 21.4 (2011), pp. 283-301. DOI: 10.1615/AtomizSpr. 2011002760.

[34] Desjardins, Olivier, McCaslin, Jeremy, Owkes, Mark, and Brady, Peter. "Direct Numerical and Large-Eddy Simulation of Primary Atomization in Complex Geometries". In: Atomization and Sprays 23.11 (2013), pp. 1001-1048. DOI: 10.1615/AtomizSpr. 2013007679.

[35] Subramaniam, S. "Statistical representation of a spray as a point process". In: Physics of Fluids 12.10 (2000), pp. 2413-2431. DOI: 10.1063/ 1.1288266 .

[36] Subramaniam, S. "Statistical modeling of sprays using the droplet distribution function". In: Physics of Fluids 13.3 (2001), pp. 624-642. DOI: $10.1063 / 1.1344893$.

[37] Corrsin. Spectra and Diffusion in a Round Turbulent Jet. Tech. rep. 8. 1943, pp. 399-405.

[38] Antonia, R. A., Anselmet, F., and Chambers, A. J. "Assessment of local isotropy using measurements in a turbulent plane jet". In: Journal of Fluid Mechanics 163.-1 (1986), pp. 365-391. DOI: 10.1017 / S0022112086002331.

[39] Hussein J. Hussein, Steven P. Capps, and William K. George. "Velocity measurements in a high-Reynolds-number, momentum-conserving, axisymmetric, turbulent jet". In: Journal of Fluid Mechanics 001.258 (1994), pp. 31-75. 
[40] Sadeghi, H. and Pollard, Andrew. "Axial Velocity Spectra Scaling in a Round, Free Jet". In: Proceeding of THMT-12. Proceedings of the Seventh International Symposium On Turbulence, Heat and Mass Transfer Palermo, Italy, 24-27 September, 2012 November (2014), p. 11. DOI: 10.1615/ichmt.2012. procsevint sympturbheattransf pal . 1220.

[41] Craske, John, Debugne, Antoine L.R., and Van Reeuwijk, Maarten. "Shear-flow dispersion in turbulent jets". In: Journal of Fluid Mechanics 781 (2015), pp. 28-51. DOI: 10.1017/jfm.2015.417.

[42] Tong, Chenning and Warhaft, Z. "Passive Scalar Dispersion and Mixing in a Turbulent Jet". In: Journal of Fluid Mechanics 292.-1 (1995), pp. 1-38. DOI: $10.1017 /$ S0022112095001418.

[43] STANLEY, S. A., SARKAR, S., and MELLADO, J. P. "A study of the flow-field evolution and mixing in a planar turbulent jet using direct numerical simulation". In: Journal of Fluid Mechanics 450.January 2002 (2002), pp. 377-407. DOI: 10.1017/s0022112001006644.

[44] Trask, Nathaniel, Schmidt, David P., Lightfoot, Malissa, and Danczyk, Stephen. "Compressible Modeling of the Internal Two-Phase Flow in a Gas-Centered Swirl Coaxial Fuel Injector". In: Journal of Propulsion and Power 28.4 (2012), pp. 685-693. DOI: 10.2514/1.b34102.

[45] Du, Wei, Bao, Xiaojun, Xu, Jian, and Wei, Weisheng. "Computational fluid dynamics (CFD) modeling of spouted bed: Influence of frictional stress, maximum packing limit and coefficient of restitution of particles". In: Chemical Engineering Science 61.14 (2006), pp. 4558-4570. DOI: $10.1016 / \mathrm{j}$.ces . 2006.02.028.

[46] Dukowicz, John K. "A particle-fluid numerical model for liquid sprays". In: Journal of Computational Physics 35.2 (1980), pp. 229-253. DOI: 10.1016/0021-9991(80)90087-X.

[47] Salvador, F.J., Ruiz, S., Crialesi-Esposito, Marco, and Blanquer, Ignacio. "Analysis on the Effects of Turbulent Inflow Conditions on Spray Primary Atomization in the Near-Field by Direct Numerical Simulation". In: International Journal of Multiphase Flow 102 (2018), pp. 4963. DOI: $10.1016 /$ j.ijmultiphaseflow.2018.01.019. 


\section{Chapter 5}

\section{Influence of integral length scale on primary atomization}

In Chapter 3 the Synthetic Boundary Condition (SBC) have been described and it appears that three main quantities can be tuned, namely the integral length scale $L$, the turbulence intensity $I$ and the Reynolds tensor $a$. From a practical standpoint, most of the works proposed in literature are acting on the value of $L$, while $I$ is usually taken to be $5 \%$ and $a$ is tuned in order to have homogeneous turbulence (at least, this is according to the information provided in literature). For example, the change of $L$ is visible in [1-3], although the different injection condition makes impossible to draw physical insight on the matter. In this context, the present chapter will address the influence of the parameter $L$ on primary atomization, with an emphasis on the morphological analysis and the droplet generation.

\subsection{Introduction and case description}

The main objective of this thesis is to provide an insight on the primary atomization in the early spray formation region, usually quantifiable in high speed sprays between $2-3 \mathrm{~mm}$ of distance from the orifice. In this region many types of atomization occur, starting from the mushroom tip breakup, followed by atomization induced by the aerodynamic forces on the spray core.

Very few experimental works are available on near field visualization [47], as already discussed in Chapter 1; furthermore to the best of the author 
knowledge, no experiments at low speed (corresponding to low injection pressures) are available, which is mandatory for DNS simulations. For this reason, the liquid/gas parameters, as well as the geometrical parameters were chosen according to a Spray A usual configuration [8], where a low speed Dodecane jet is injected in a pressurized nitrogen vessel. Experiments on Spray A case have been conducted extensively throughout last years and it is likely to be the best experimental configuration for future validation of simulation results. The parameters used are resumed in Table 5.1. These properties have been set during the whole thesis as strongly representative of many applications. Especially, the density ratio is typical of many combustion processes, where the combustion chamber is pressurized in order to favor the thermodynamic conditions for combustion to occur.

\begin{tabular}{|c|c|c|}
\hline Parameter & Values & Units \\
\hline Injector diameter & 0.09 & $\mathrm{~mm}$ \\
\hline Fuel viscosity & $1.34 \cdot 10^{-3}$ & $\mathrm{~Pa} \cdot \mathrm{s}$ \\
\hline Fuel density & 750 & $\mathrm{~kg} / \mathrm{m}^{3}$ \\
\hline Fuel Mean Velocity & 100 & $\mathrm{~m} / \mathrm{s}$ \\
\hline Fuel/Nitrogen Surface Tension & $2.535 \cdot 10^{-2}$ & $\mathrm{~N} / \mathrm{m}$ \\
\hline Nitrogen Viscosity & $1.85 \cdot 10^{-5}$ & $\mathrm{~Pa} \cdot \mathrm{s}$ \\
\hline Nitrogen Density & 22.8 & $\mathrm{~kg} / \mathrm{m}^{3}$ \\
\hline
\end{tabular}

Table 5.1: Simulation physical parameters

As known from the theory of self-similarity in sprays and jets [9], the velocity profile should assume a Gaussian shape only for $x / D>25 \div 30$, where $x$ is the spray axial penetration and $D_{n}$ the nozzle diameter. In order to comply with this hypothesis, the turbulence inside the nozzle needs to be simulated and used as a boundary condition in the DNS simulation, in order to prompt the atomization process and therefore leading to a axial breakup that will ultimately allow to generate a Gaussian profile. This consideration also guides, in a first instance, in the design of the simulation domain. In order to replicate and verify this behavior, the domain has been set to 2.34 $\mathrm{mm}, 0.6 \mathrm{~mm} 0.6 \mathrm{~mm}$ in $x, y$ and $z$ respectively. As suggested in [10] the cells are cubes of $2.34 \mu \mathrm{m}$, resulting in approximately 65.5 millions of cells.

As reported in [1], the turbulent length $(L)$ scale can be assumed as $10 \%$ of the diameter and the turbulent intensity $(I)$ might be assumed as a $5 \%$ of the axial mean velocity. Still, the turbulent length scale may change significantly with the nozzle geometry: for this reason, one of the main goal of this study is to assess the effects on the primary atomization of the parameter $L$ by means of 3 DNS cases, described in Table 5.2. 


\begin{tabular}{|c|c|c|c|}
\hline & $L$ & $R e_{c}$ & $R e_{b}$ \\
\hline case 0 & 0 & 5037 & 5037 \\
\hline case 1 & $0.1 D$ & 6700 & 5037 \\
\hline case 2 & $0.17 D$ & 6700 & 5037 \\
\hline
\end{tabular}

Table 5.2: Inlet turbulence model parameters for all the cases simulated

As far as the author's knowledge goes, no previous researches on the topic are available where $L$ have been investigated by a parametric study. Furthermore, as most studies on internal nozzle presents velocity fluctuations that are comparable to the one used here, the main effort is concentrated on the lengthscale $L$. The parameter $L$ acts directly as a source for turbulent energy for the spray. In fact, the formation of larger turbulent structures, such as eddies and vortexes, generates a cascade effect that distributes the energy to the lower scales. As it will be shown in the following section, the local atomization is highly influenced by the wavelength of the local disturbances, therefore the $L$ parameter is a key factor in determine the droplet breakup regime.

In the present chapter, first a comparison of the three cases will be made in Section 5.2 aiming to highlight the differences introduced by the different boundary conditions depicted in Table 5.2. Section Section 5.3 will focus the analysis on the spray morphology, while Section 5.3.1 will discuss the effects of the different axial velocities in the 3 cases. The analysis of the physical processes generating the atomization will be addressed in Section 5.4. The droplet analysis, in terms of diameter, distribution and physical properties will be studied in Section 5.5. Finally, some considerations on the basic properties of the Gaussian profile has been made in Section 5.6.

Figure 5.1 shows a schematic representation of the main spray elements analyzed in this thesis. The external non-perturbed length, $L_{n p}$, coincides with the length of the spray region where the liquid core maintains a pseudocylindrical shape, before the atomized region. The intact core length, $L_{i c}$, is the statistical length that determines the distance between the injector nozzle outlet and the point in the spray axis in which the probability of finding liquid is below $99.9 \%$ (hence the axial liquid column stop being continuous). The ligaments will be identified by their diameter, $D_{l}$, and their external perturbations will be described by the wavelength, $\lambda_{l}$, that the liquid interface forms due to external disturbances. The usage of the symbol $\lambda_{l}$ As the spray starts atomizing, an atomized region is formed, where the spray core is hidden by the droplets cloud. This droplets cloud is usually used to define the spray cone angle in near-field visualization experiments. The tip region is the region 


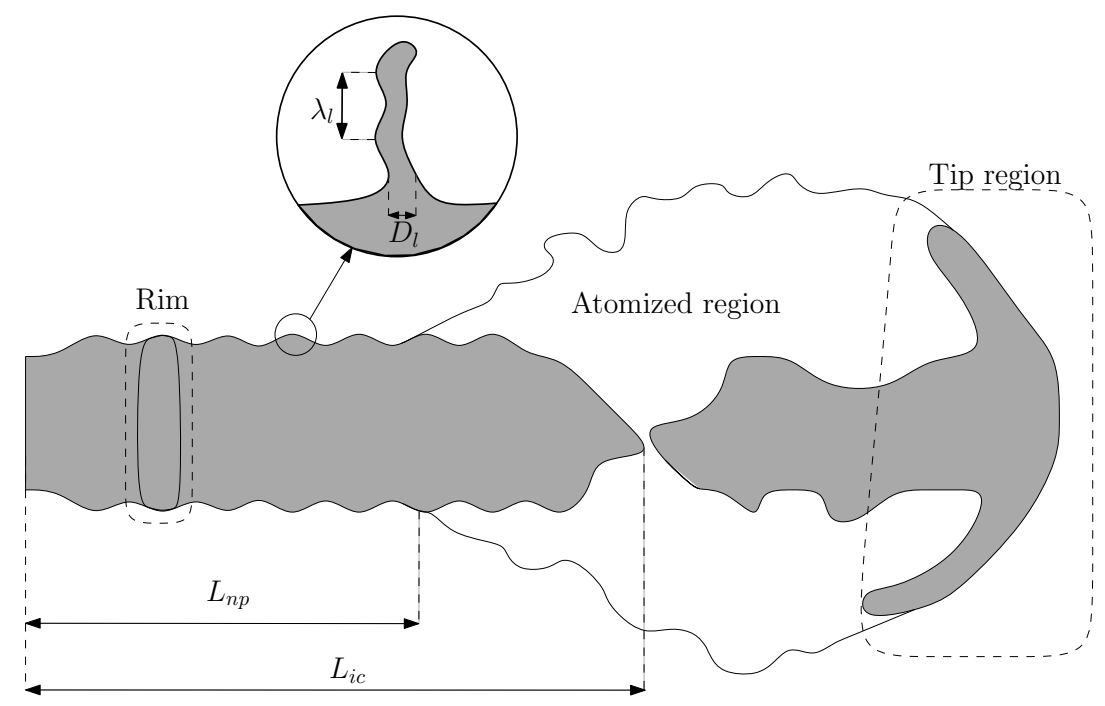

Figure 5.1: Schematic representation of the spray, presenting the nomenclature used in the analysis.

where the cylinder spray is bent by its impact with calm air. Finally, the irregularity created on the external liquid core (visible in the external nonperturbed region) are called rims. This liquid structures are of fundamental importance as they are directly involved in the ligaments formation.

\subsection{Effects of the Synthetic Boundary Conditions}

As a first qualitative validation of the algorithm used, Figure 5.2 highlights the changes generated by the synthetic turbulence boundary condition, presented in Chapter 3 for the 3 cases ( Table 5.2). This figure shows the vorticity effects generated by the inlet turbulence on the liquid surface (here represented with VOF isosurface) over the spray external non perturbed region at $t=20 \mu \mathrm{s}$.

Figure 5.2(a) shows the vorticity field on a $2 \mathrm{D}$ section. The synthetic turbulence in case 0 to 2 increases the area influenced by a strong vorticity field, as already observed in Section 5.1; in particular, is clear how cases 1 and 2 present a strong turbulent field and how the area of influence of the turbulent structures grows with the parameter L. Figure 5.2(a) highlights how, for higher values of $L$, the structures generated within the spray in the external non perturbed region increase their size, the region of influence and their magnitude. It is notable that in case 2, the structures reach the fluid 


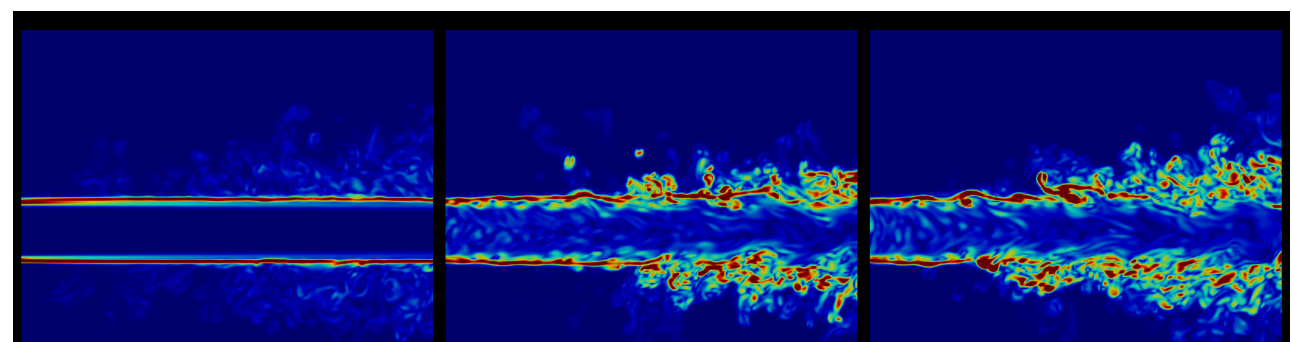

Vorticity $[1 / s]$

0

$2.5 e+06 \quad 5 e+06 \quad 7.5 e+06 \quad 1 e+07$

(a) Vorticity in the spray core in the external non perturbed region. Left to right case 0,1 and 2 .

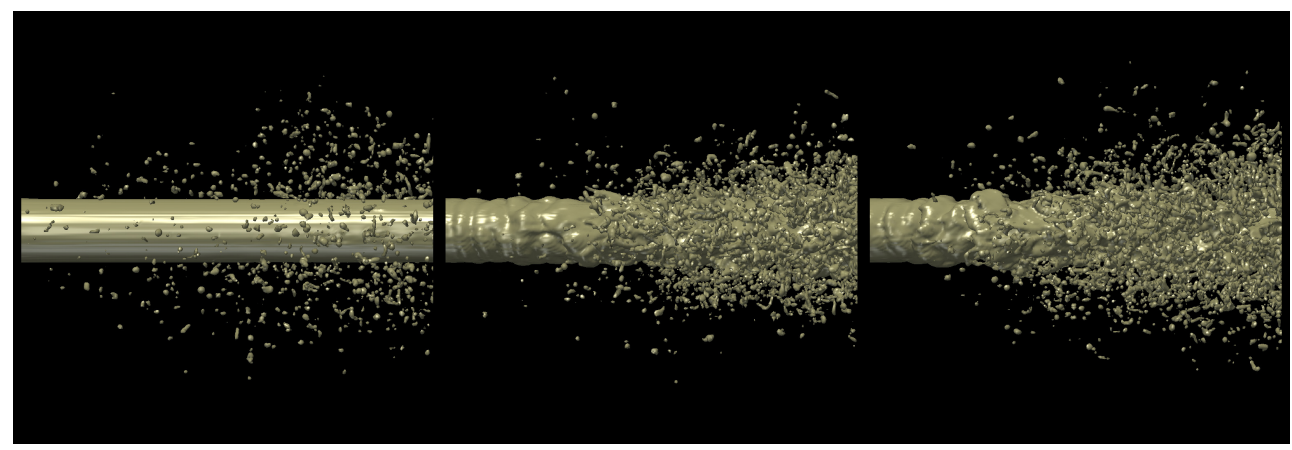

(b) VOF renderization in the external non perturbed region.

Figure 5.2: Spray behavior in the transient spray under the synthetic boundary condition effects. Left to right case 0, 1 and 2.

surface faster than in case 1, leading to consequent larger portions of the liquid core been affected by irregularities, as shown in Figure 5.2(b). On the other hand, case 0 shows a plane aspect, leading to the conclusions that in this region of the spray the aerodynamic forces are not sufficient to generate perturbation on the liquid surface. This behavior observed in case 0 lead to an atomization process almost only exclusively promoted by the spray tip breakup.

Figure 5.3 shows, in the upper part of the spray, a scheme of the eddies formation, while in the lower part the air relative velocity with respect to 


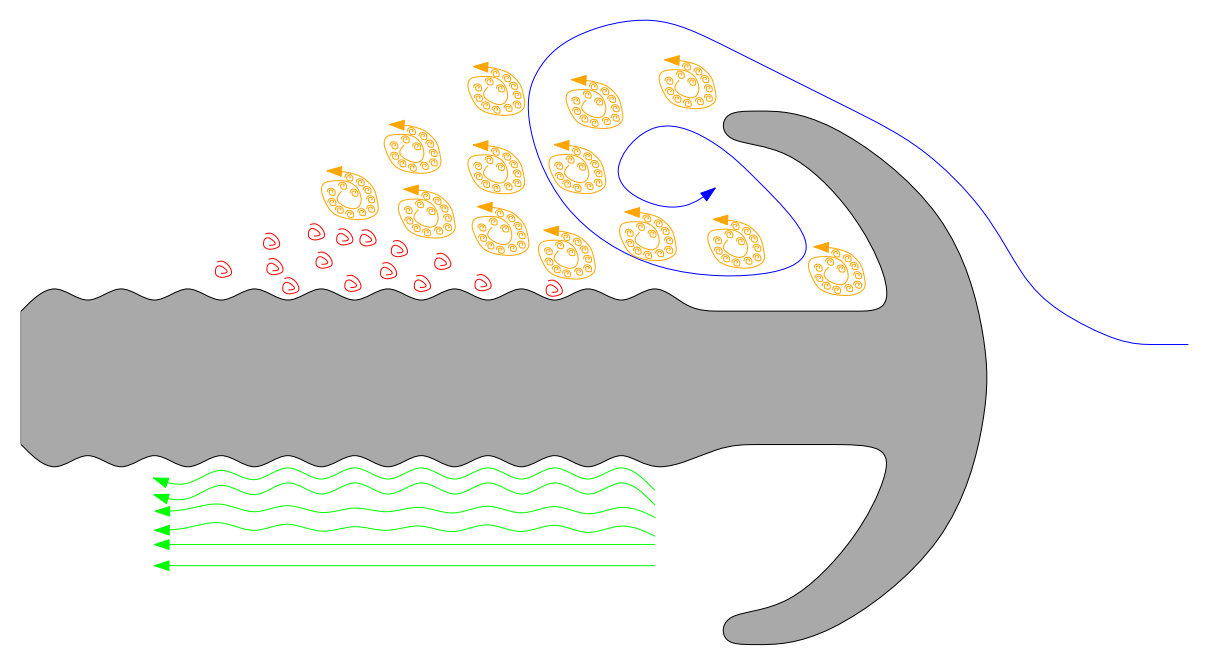

Figure 5.3: Simplified scheme of the distribution of aerodynamic drag forces and vorticity field around the spray tip and core

the spray. This simple scheme can help to explain the three different effects generated by the turbulent inlet condition on the atomization process, which are described in the following:

(1) The synthetic turbulence described in Chapter 3 generates effects in the three dimensions and deforms the liquid core from the inside-out. The velocity propagation in the core, allows the formation of small size rims along the liquid core in the first milliseconds of the spray penetration.

(2) The tip bends due to its impact with calm air, generating the mushroom shape peculiar of the tip region. This liquid-gas interaction generates, at first, an eddy structure of large size (even larger than the nozzle diameter), represented as a single blue line in Figure 5.3. This large turbulence structure is a toroid in three dimensions. The large eddy contributes to both modify and perturb the liquid region below the spray tip. While doing so, the structure decomposes into smaller eddies that propagates in the opposite direction with respect to the spray penetration (the yellow and red structures in Figure 5.3). As this smaller eddies are moving from the tip region towards the liquid core, they acts as external disturbance for the core liquid surface, while amplifying the rim size and modifying the local velocity field, eventually leading to the core breakup (defined by the intact core length). 
(3) As the spray keeps penetrating, the effects of the vortex cascade generated by the larger tip eddy are mitigated, due to the higher distance a eddy has to travel to reach the nozzle outlet. As a matter of facts, the vorticity effects on the spray core almost disappear after a certain spray penetration. Once this level of penetration has been reached, the only force acting on liquid core is the aerodynamic drag force. As a well known phenomena, the drag depends on the relative velocity between the two moving bodies or, in this case, between the two phases as showed in Figure 5.3 with green lines. When a rim is formed (by the mechanism depicted in (1) without the contribution of the effects explained in (2)) the green line in Figure 5.3 are bended and the drag forces generate a radial stress that acts like a disturbance. This radial disturbance is way more important in the spray core deformation than the simple axial stress generated in absence of rims.

Figure 5.4 shows a 2D section of the simulation domain for case 0 (top) and case 1 (bottom). The liquid phase is represented and the field is colored by the vorticity field; finally, displayed on the background, vortex lines obtained from the Line Integral Convolution (LIC) technique are displayed. The comparison is made at $10 \mu s$ after injection starts. Here the hypothesis (1), (2) (3) on how the velocity fields develops and affects the atomization process can be numerically observed. At this time, the spray penetration is still reduced (when compared to the whole domain) but it is quite evident the difference that effects (2) and (3) are inducing on the spray core and on the atomization process (especially for case 0). Furthermore, at this instant, case 1 is still showing an almost intact liquid core, which make easier to apply the analysis of the effects (2) and (3).

In a first place, the turbulence macro-structures developed in case 0 and case 1 will be analyzed, with the objective of drawing considerations on how dynamics involved with effect (2) are affected by the use of the synthetic turbulence at the inlet. In both cases, in Figure 5.4, the larger eddy (described in effect (2) and represented with a blue line in Figure 5.3) can be clearly identified by the the vortex lines around the spray tip, bended toward the spray axis. Also, the larger structure shows the same size, although case 1 display a more chaotic behavior, obviously related to the synthetic inlet boundary conditions. This first observation lead to the conclusion that the synthetic inlet turbulence does not affect the large scale motion of the fluid (except for the spray penetration, that will be addressed later in section 5.3.1) as it is expected. Nevertheless, Figure 5.4 shows a significant variation in both the vorticity field, as well as in the shape of the liquid spray. Case 0 shows 
a high intensity region for vorticity only in the immediate proximity of the spray tip, suggesting how the development of turbulence in this case is strictly related to effect (2), confirming that effect (3) is still not affecting at this stage of the penetration. On the other hand, case 1 displays many dispersed high vorticity regions: in this case a more intense turbulence is generated close to the surface instability. This phenomena could be explained by the higher shear stress generated by the relative motion of liquid and gas when the liquid surface is bended and not cylindrical (as for case 0). For this reason, case 1 promote, in a first place, the generation of a more intense turbulence field around the spray.

Further observations could be made on how the instabilities are generated in the external non-perturbed length region. At this time, the spray is sufficiently far from the nozzle so that the effects that mechanism (2) is generating are mitigated and only effects (3) and (1) (for case 1) are responsible for the spray core perturbation in the area closer to the nozzle outlet. As showed clearly in Figure 5.4, case 0 shows a very cylindrical aspect in the first part (closer to the nozzle) while the liquid core got perturbed closer to the spray tip (due to effect (2)). Again, in both cases a higher values of vorticity can be observed close to the surface corrugation, while the highest absolute vorticity value can be located close to the larger eddy's center. An insight on how the turbulence behavior relates to the surface corrugations is given in Figure 5.5. This figure shows, for both, case 0 and 1 , the contour of the second invariant of the velocity gradient tensor, representing hairpin vortexes. Both, in case 0 and 1, a significant amount of hairpins are generated close to the spray tip. In order to isolate the effects of the synthetic turbulence, the behavior of the vortexes will be first studied for case 0 , where only effect (2) is responsible for the generation of turbulent structures. Similarly to the literature results $[11,12]$, case 0 displays the ongoing formation of axisymmetric structure in the spray tip, normal to the penetration axis. As the spray tip penetrates, these vortexes are distorted by the velocity field behind the tip. The vortexes are re-oriented and finally, when they are sufficiently far from the tip, they are almost parallel to the penetration axis. The absence of structures in the region close to the nozzle suggests that, in lack of a highly turbulent environment, the vortexes quickly dissipate. Case 1 displays some major differences when compared to case 0 . As the synthetic inlet turbulence is imposing a fluctuating velocity in the three directions, the spray tip will display a strongly asymmetric tip when compared to case 0 (see Figure 5.4). Therefore, the dispersion of the turbulent structures will have a stronger radial component, while their formation will not be as axisymmetric as for case 0. Furthermore, the presence of corrugation on the spray surface in the external non perturbed 
length, due to the radial components of the injected turbulence, promote the generation of rims (effect (1)) that, due to the shear with the gas phase cause the generation of turbulence structures. This structures are similar to horseshoe hairpin, and ensure the conservation of the turbulence even where the disturbance generated by the spray tip cannot reach the liquid core. In other words, in case 0 the vortexes spreading from (2) are not able to reach the nozzle outlet, meaning that, for case 0 , only effect (3) is acting on the spray liquid core at the immediate nozzle outlet. On the other hand, case 1 shows a significantly perturbed liquid core region, given by a mix of effects (1) and (3) explicated above. Consequently for case 0, in Figure 5.4 the liquid surface is perturbed only in the areas where hairpins can be found in Figure 5.5

Finally, the liquid core in the tip region shows a more chaotic structure and a first discontinuity in the axial mass concentration appears. The sequence of events (1) (2) (3) in case 1 generates a more severe perturbation on the liquid surface that ultimately leads to the axial breakup as the spray penetrates. Even when the liquid surface instabilities generated by (2) are far from the nozzle, effect (1) is still sustaining and even amplifying the rims in this region, increasing the aerodynamic stress generated by effect (3). Once again, the axial breakup helps interpreting the results, quantifying that the generation of rims and, consequently, of radial forces is way more significant in the spray formation than axial shear stress induced by aerodynamic interaction between calm air and liquid.

\subsection{Observation on the Spray Morphology}

Figure 5.6 shows the external aspect of the spray at $t=20 \mu \mathrm{s}$. As it can be clearly noted, the higher the turbulence induced, the sooner the atomization process starts, shortening the external non-perturbed length. When comparing all cases, case 2 displays the formation of a earlier atomization region, due to the rims created in the external non-perturbed legth region that creates a dense cloud of droplets in the near-nozzle field. On the other hand, it is evident that in case 0 the external non-perturbed length maintain an almost exact cylindrical shape up to the spray tip, due to the low nitrogen density and the low injection velocity. As a confirmation of the synthetic turbulence influence on the atomization process, in case 1 the droplet cloud (that will eventually define the spray angle) starts in an axial position between case 0 and case 2. Similar results have been obtained in [13] for a nozzle of similar size in different injection conditions. 

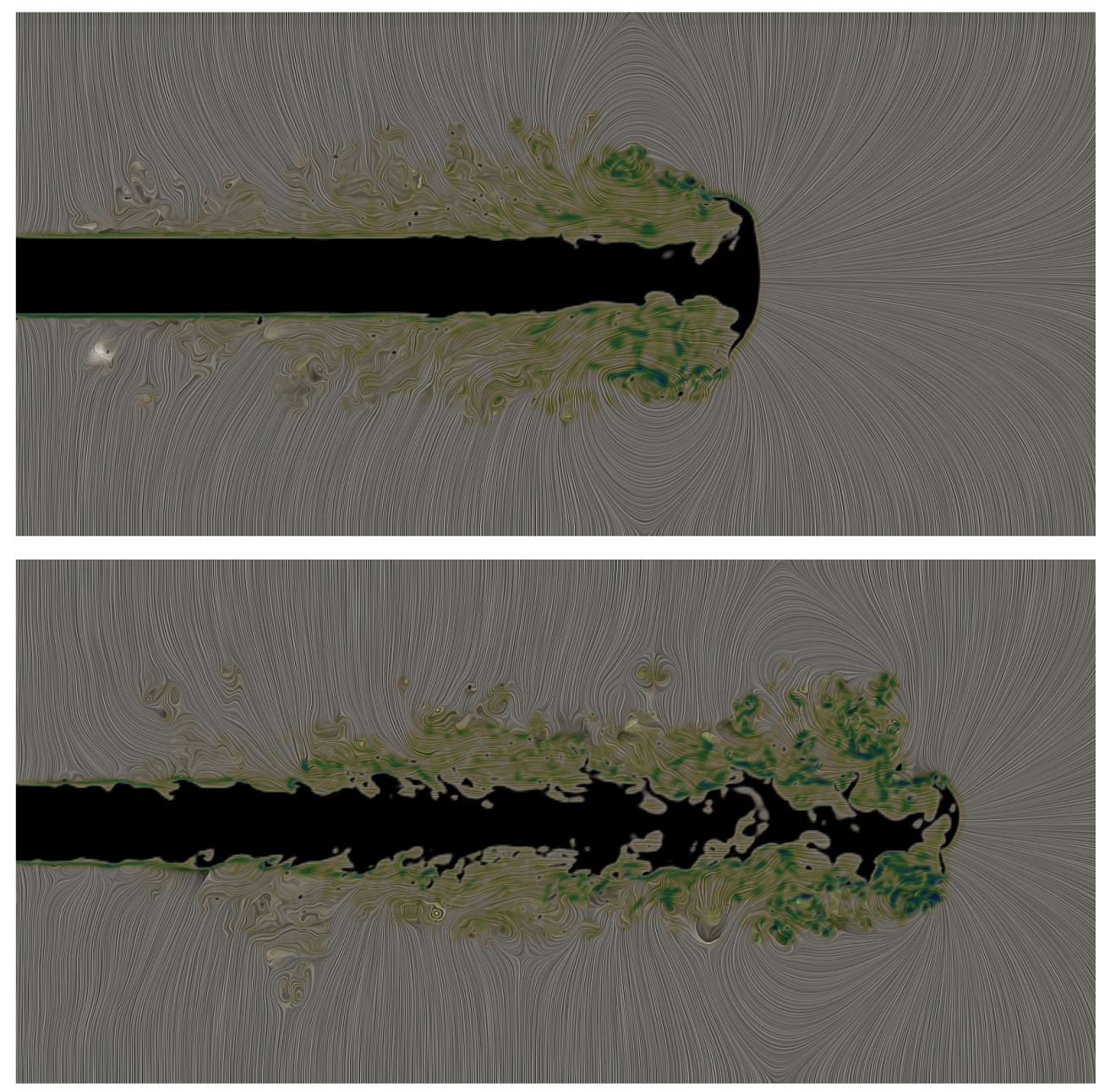

\section{Vorticity [1/s]}

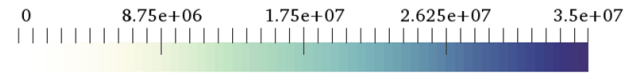

Figure 5.4: Case 0 and 1 at $10 \mu \mathrm{s}$. 

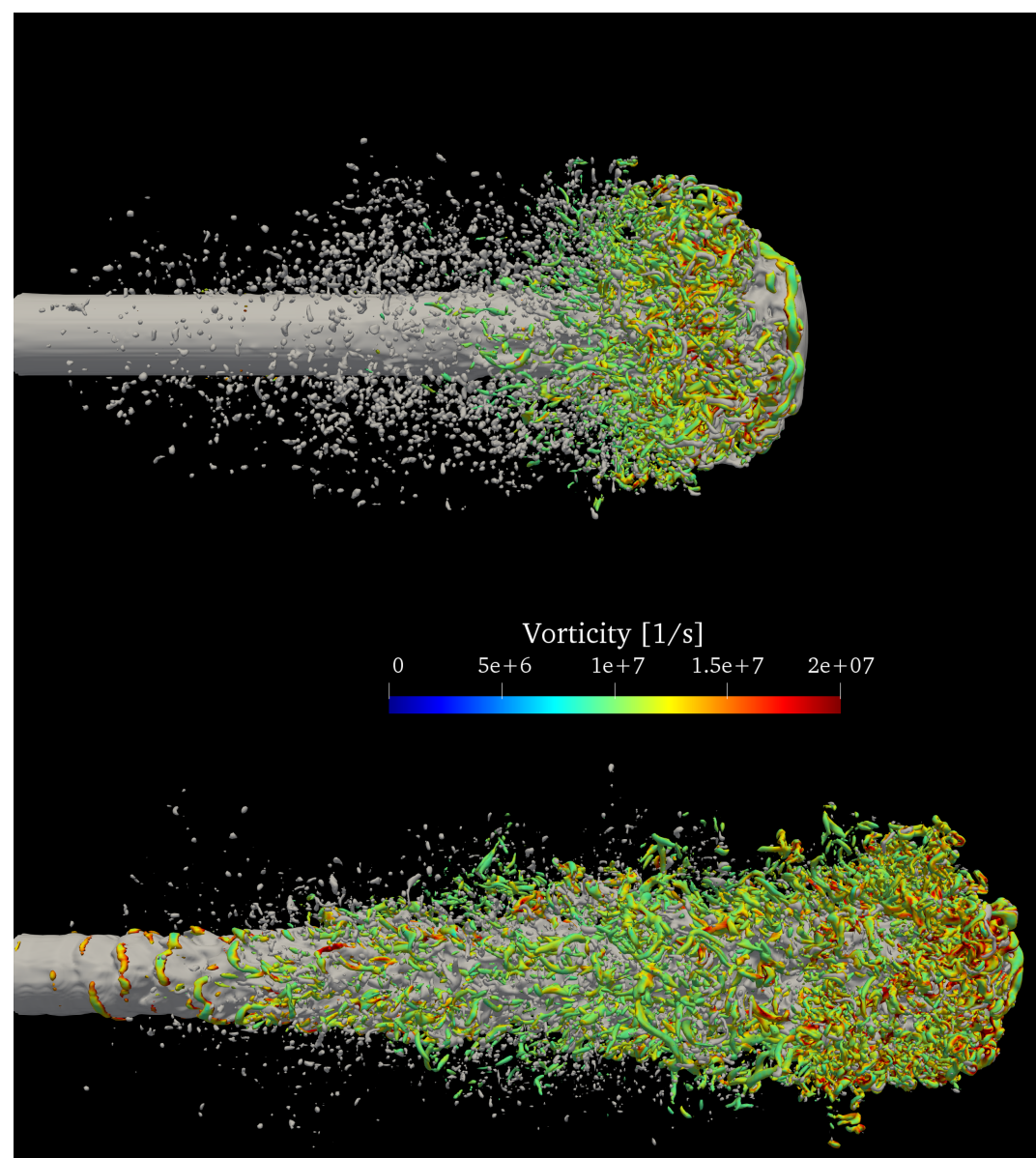

Figure 5.5: Turbulent structures detected through the Q-criterion for cases 0 and 1 at $10 \mu \mathrm{s}$. 


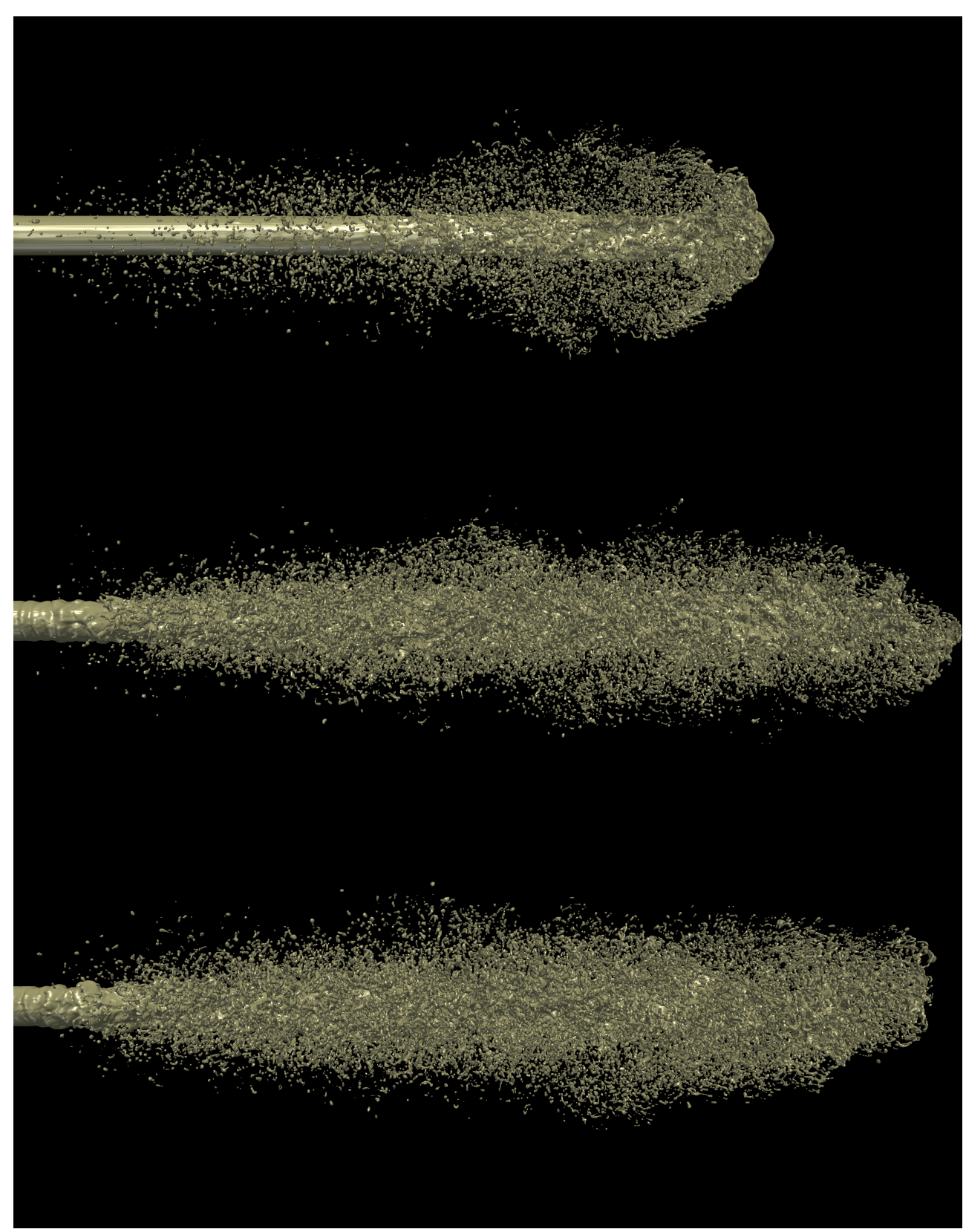

Figure 5.6: External aspect of the injected spray at $t=20 \mu \mathrm{s}$. From top to bottom, case 0, 1 and 2 


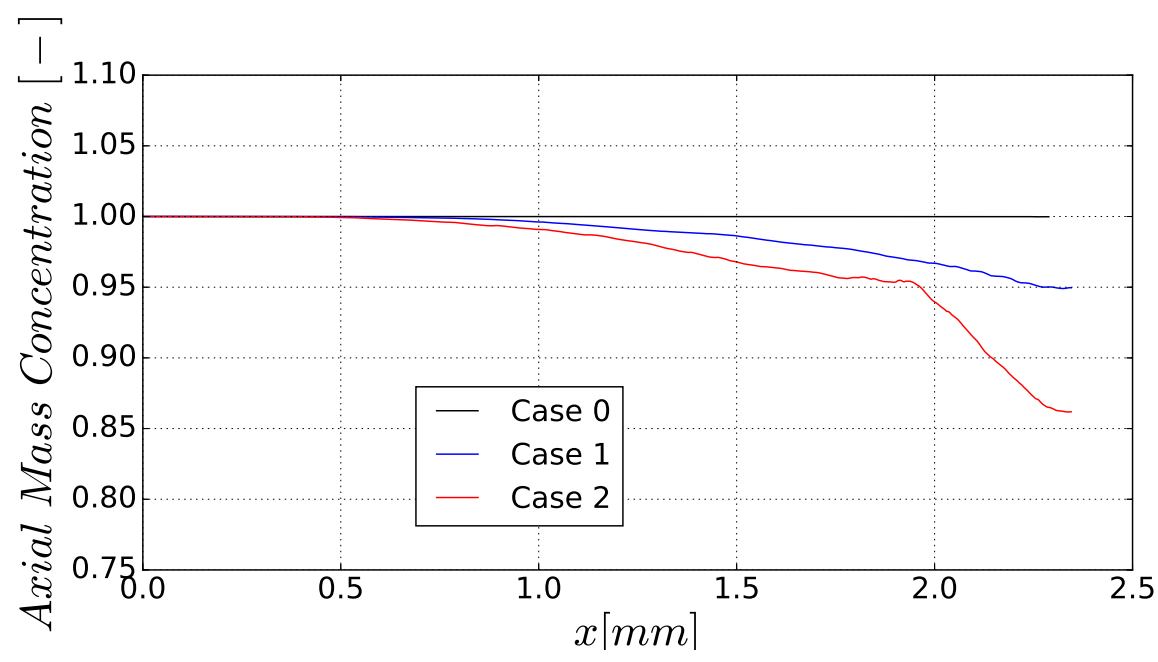

Figure 5.7: Axial time-averaged mass concentration

The different behavior among the three cases can be quantified by means of the liquid mass concentration in the axis, calculated as :

$$
m_{c}=\frac{\rho_{l} \cdot C}{\rho_{l} \cdot C+\rho_{g} \cdot(1-C)}
$$

Figure 5.7 shows the time-averaged mass concentration in the spray axis. Once the spray is stabilized for the three cases, $m_{c}$ is used to characterized the intact core length ( Figure 5.1), which is directly related to the atomization intensity. As it can be seen in Figure 5.7, in the case 0, due to the poor atomization, the mass concentration in the axis is not perturbed, showing a value of 1 (pure liquid) in the spatial window analysed (up to $2.34 \mathrm{~mm}$ ). However, in case 1 and especially in case 2, the intact core length drastically decreases as a result of the higher turbulence induced in the nozzle exit. This behaviour quantifies the earlier qualitative explanation of Figure 5.6, where the external non-perturbed length increases with the inlet turbulence lengthscale.

It is interesting to notice that the case with the highest turbulence level (namely case 2) in Figure 5.2 experiences a core deformation that creates rims very close to the nozzle outlet. This generates an increase in the vorticity and in the local velocity field at the interphase between liquid and air (Figure 5.2(a)), finally increasing the atomization, as showed in Figure 5.6. The rims are almost non existent in case 0 leading to a low vorticity field and, 
consequently low atomization, mainly focused in this case around the spray tip where droplets are separating from the ligaments.

Finally, Figure 5.7 allows to estimate the liquid core length. Taking as a threshold an axial mass concentration of 0.97 , case 1 and 2 present a liquid core length of 1,47 $\mathrm{mm}$ and $1.91 \mathrm{~mm}$ respectively, while this parameter cannot be assessed for case 0 .

\subsubsection{Consideration on the Axial Spray Penetration}

The results presented in Figure 5.6 highlights an interesting aspect about penetration, as the 3 cases seem to show different axial position of the spray tip at the same timestep. A first remark between case 0 and cases 1 and 2 involved the axial Reynolds $\left(R e_{c}\right)$ : as reported in Table 5.2, although the 3 cases have the same inlet mass flow rate (same average spray velocity at the nozzle outlet), the velocity distribution drastically changes as showed in Chapter 3 . Therefore, it is assumable that axial penetration is mostly a function of $R e_{c}$.

Finally, the difference in penetration between case 1 and 2 can be found in the different rate of atomization between the two cases. As showed in Figure 5.7, case 2 atomizes more than case 1, causing a more atomized tip region as well. This behavior is actually responsible for the difference showed in the render in Figure 5.6, as the total momentum needs to be conserved. In fact it has been widely proven in sprays that the largest the angle (and therefore the spray atomization) the lower the penetration will be.

\subsection{Observations on the atomization process}

Shinjo and Umemura [11] and Shinjo et al. [12] highlight how the ligaments formation could occur as a consequence of a liquid detachment from the tip region or from the liquid core. In the first case, the ligament are created by the shear generated by the tip region macro-vortex (blue line in Figure 5.3), while in the second scenario, the ligaments formation is related to the aerodynamic force (effect (3)), increased by the smaller eddies generated from the macro vortex (effect (2)). The analysis in [11] also highlights how one of the most significant dynamic in spray atomization is given by droplets impacting the rim surface, due to the large eddy produced by the spray tip that drove the droplets towards the spray core. A significant insight on this mechanism is given by Jarrahbashi et al. in [14]. Here the authors highlights how the shear stress, described in [15], is itself sufficient to generate counter-rotating hairpins that are responsible for the formation of ligaments and, subsequently for the primary atomization. 


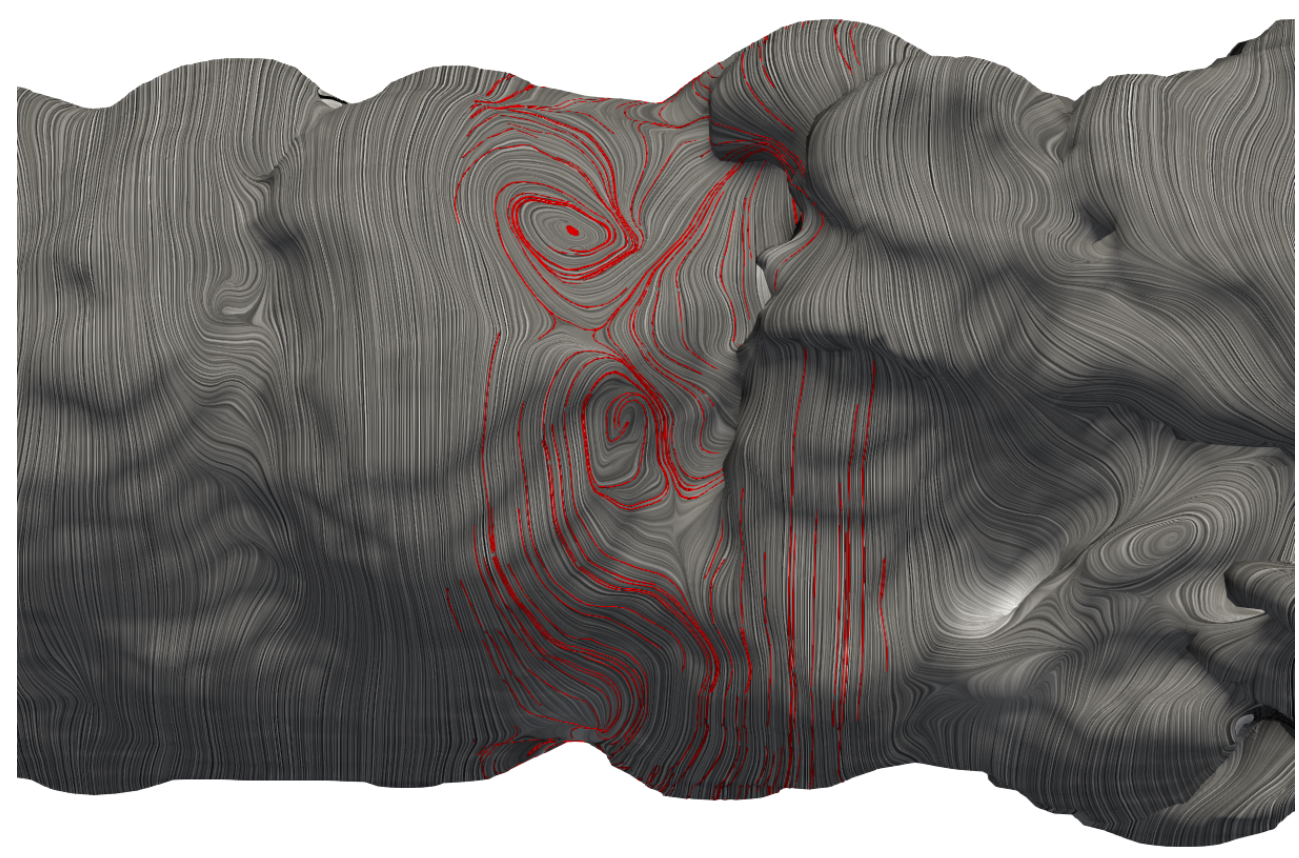

Figure 5.8: Detailed view of the vortex lines over the spray core at $t=65 \mu \mathrm{s}$. In red, the vortex lines belonging to the same rim are highlighted.

Figure 5.8 shows a zoomed view of the isolated spray core in the external non-perturbed length region. The vortex lines projected over the spray core are displayed in black. Finally, in analogy with [14], the vortex line belonging to the same rim are coloured in red. Figure 5.8 clearly displays a more chaotic surface than the one displayed in [14], nevertheless, the presence of counterrotating vortices is evident in the red lines and, in general, along the whole region. On the other hand, assume that the rim tear (that ultimately leads to the formation of ligaments) is only due to the aerodynamic shear stress is somehow hard to prove in the three cases tested in the present work. Referring to Figure 5.6, is clear that the aerodynamic forces have not yet generated a sufficient tension on the liquid surface to trigger the formation of instabilities (similar results have been found in [11]). The real phenomena occurs in such a way that the external non-perturbed length is not able to keep the cylindrical shape for such a length as in case 0 or in $[11,12]$, rather it shows a behavior closer to the one in cases 1 and 2, as showed in [16], due to the obvious perturbation that the fluid undergoes at the nozzle exit. For these reason, the rim/ligaments breakup is more likely to occur due to the combination 
of aerodynamic shear stress and instabilities, that may be triggered both by small vortexes or by the impact of the droplets recirculated by the main larger eddy. In any case, in such a complex scenario, it is difficult to separate effects, therefore future studies on this subjects are required.

Figure 5.9 displays the evolution of the spray in a $1 \mu \mathrm{s}$ interval for case 1. Four zones can be can be identified, depending of the values the axial mass concentration showed in Figure 5.7. Obviously, the division in areas will change for each case, depending on his axial mass concentration. On the left side of the red line, the external non-perturbed length can be identified. In this zone no major effect of the aerodynamic drag can be identified (effect (3) in Section 5.2) and the external shape of the liquid core is almost uniquely affected by the inlet velocity profile (effect (1) in Section 5.2). This region do not show any significant ligament and therefore no atomization is observed here; such a peculiarity is easy to observe experimentally. In fact, is one of the few measurable parameter from optical measurements [7].

A second region can be observed between the red and the green line (where the axial mass concentration drops below $99 \%$ in Figure 5.7). Here the small rims created in the previous region grow due to the increase in the aerodynamic forces acting on the spray core (effect (3) in Section 5.2). This zone represents the region where atomization eventually starts and marks the border between the external non-perturbed length and the atomization region. As a matter of fact, some of the rims are already showing an atomized behaviour, due the ligaments created by the shear stress. Furthermore, the almost toroid/conical shape that the rims displays in the previous region is almost entirely lost here and a severe breakup has already start to occur. As can will be observed in Section 5.3, this region shape depends on the turbulence intensity.

A third region, between the green and the blue line, shows a region with developed atomization regimes. The limits of this region are given, on the left side, by the amplification of the wave amplitude of the instabilities generating the rims, while on the right side by the axial point on which the axial mass concentration drops below $97 \%$ in Figure 5.7. Here the atomization regime is fully developed and air-liquid interaction creates a sufficient condition to continuously support the formation of the ligaments and their instabilities, that finally determines atomization.

Finally, a last region can be analyzed on the right side of the blue line. Here the axial mass concentration on time averaged results is clearly below $96 \%$ (as well as the probability to find liquid in the spray axis) and the whole movement of the liquid parts occur on larger length scales. The main liquid 


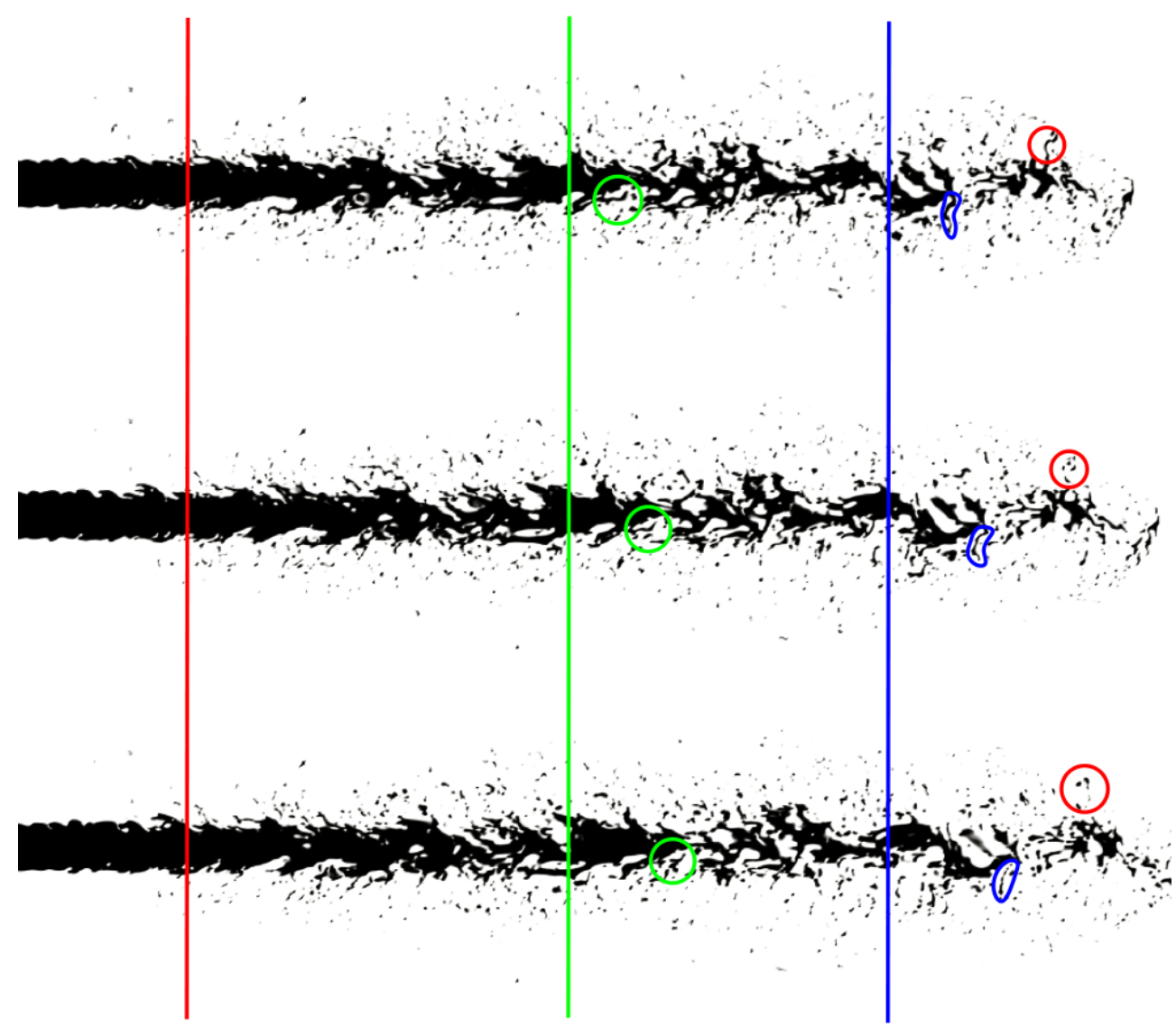

Figure 5.9: Time sequence of spray formation. The three planes show the liquid evolution at $20 \mu \mathrm{s}, 20.5 \mu \mathrm{s}$ and $21 \mu \mathrm{s}$ (respectively from top to bottom) for case 1 .

perturbation is given by the spray tip-air interaction. The eddies appear at a bigger scale (for the biggest reaching almost the size of the spray itself) and this effect leads to higher disturbance wavelength if compared to the previous zone.

Based on the analysis reported in $[17,18]$, some further analysis can be made on the type of atomization regime by studying the local Weber number $W e$, defined as:

$$
W e=\frac{\rho u_{r}^{2} D_{l}}{\sigma}
$$




\begin{tabular}{|c|c|c|}
\hline & $\lambda_{l} / D_{l}$ & $W e$ \\
\hline Red & 10.9 & 3.2 \\
\hline Blue & 7.9 & 5 \\
\hline Green & 6 & 9 \\
\hline
\end{tabular}

Table 5.3: Ligament parameters of the ligaments highlighted in Figure 5.9

where $D_{l}$ is the ligament diameter (Figure 5.1) and $u_{r}$ is the relative velocity between the liquid and the air, calculated as $u_{r}=u_{l}-u_{g}$, where the $u_{g}$ is taken per each cell at a $1.5 D_{l}$ from the ligament surface. The ligament diameter $D_{l}$ is computed using its $3 \mathrm{D}$ volume.

In order to analyze the axial evolution of the atomization process, three different ligament formation and breakup have been studied during their process, circled in Figure 5.9 in blue, red and green. Each one reflects different influences of the relative velocity field between liquid and air. In fact, it can be assumed that the farther the ligament is from the spray tip, the calmer will be the air, as it will show less influence from the tip larger eddy (effect (2) in section 5.2). This is testified by the decreasing Weber number along the spray axis: as the size of the 3 ligaments is comparable, is clear how the relative velocity between air and liquid is decreasing along the spray axis. Furthermore, is clear that most of the ligament velocity is generated by the spray liquid core, therefore this gives space to the conclusion that the relative velocity is higher in the spray region closer to the nozzle. The results shows a good agreement with the Weber numbers found for ligaments by [11].

Interesting conclusion can be drawn also on the secondary breakup and the formation of satellite droplets, based on the approach proposed in [17]. Being all the ligaments considered in this analysis in the medium wavelength Rayleigh breakup regime, this behavior is assumed to be consistent and repeatable [17]. As reported in [17], the higher the ratio between the ligament wavelength $\lambda_{l}$ and the ligament diameter $D_{l}$, the higher the ratio between the satellite droplet diameter and the main droplet diameter is. In other words, this indicates that, for sprays, secondary breakup is most likely to occur in regions where the Weber number is lower and therefore the air speed is higher.

\subsection{Analysis of droplets generation}

In order to assess the variation in the atomization process introduced by the SBC, a study of the droplet statistics was performed. The study has been done over an average time of $20 \mu s$ when the spray has reached the complete 


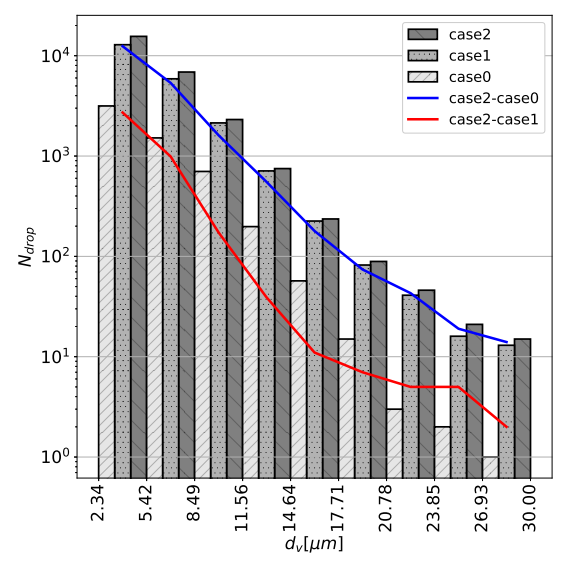

(a) Number of droplets against droplet di- (b) Number of droplets against droplet raameter
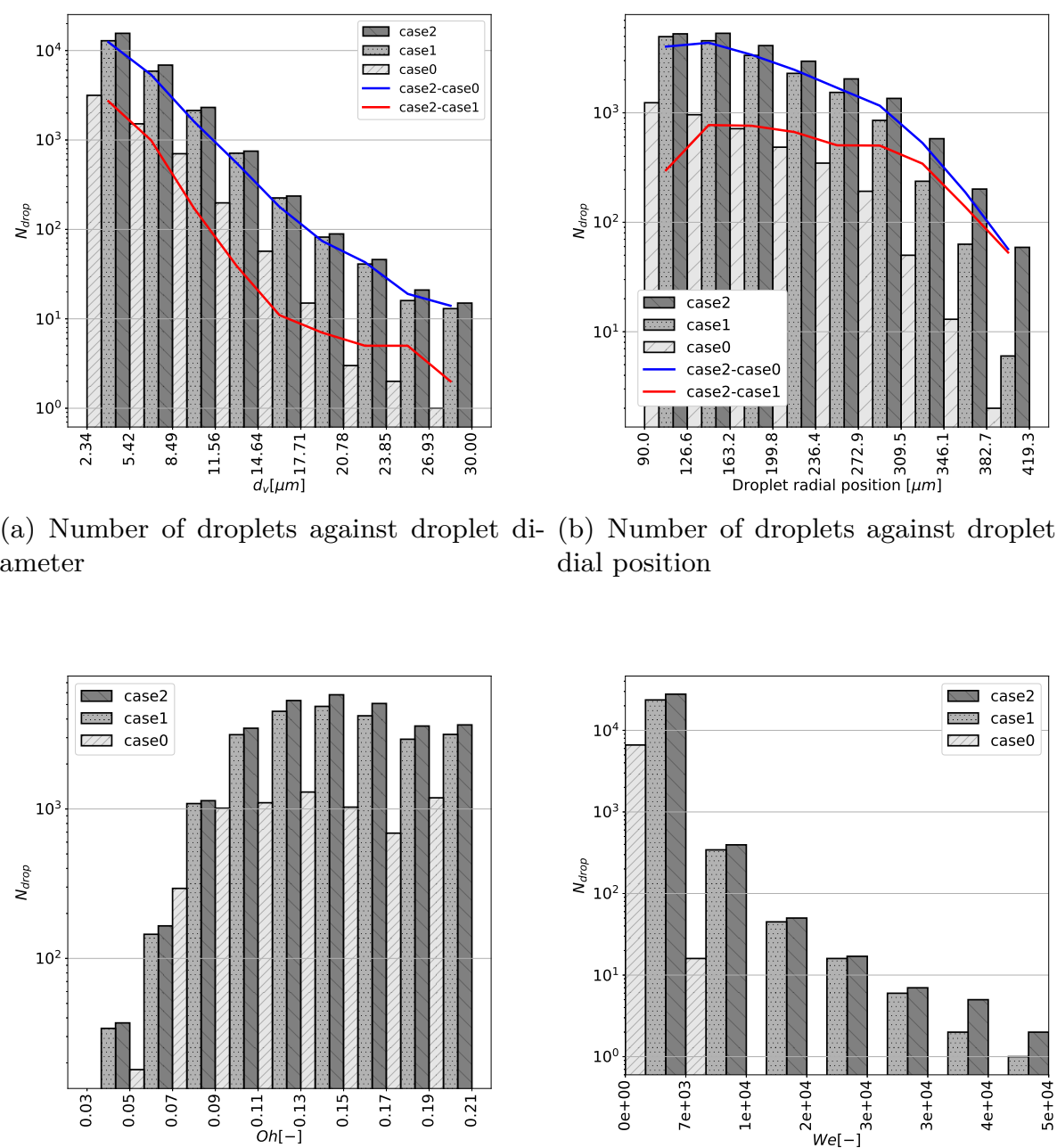

dial position

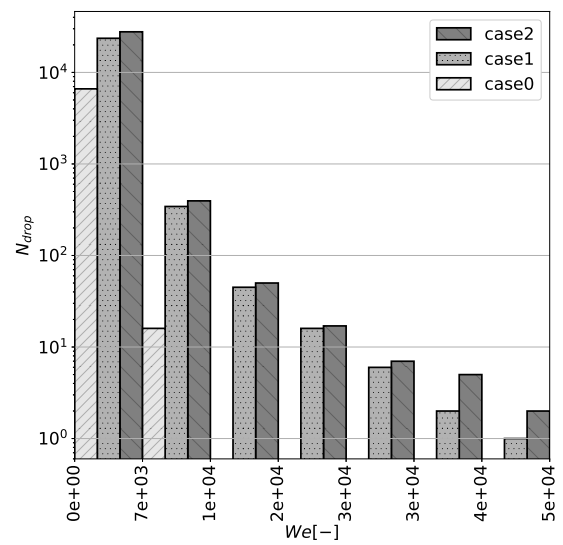

(c) Number of droplets against $O h$ number (d) Number of droplets against $W e$ number 
penetration. Such a time interval is insufficient for turbulence statistics, but it provides a significant sample time for studying the droplet behaviour, as it allows the incoming spray to reach the end of the domain for case 1 and 2 .

This study is incomplete and insufficient for drawing strong conclusions but it is an interesting quantitative way to understand the effects provided by synthetic boundary condition. In fact, due to the short total simulation time, turbulence statistics cannot be computed, therefore the droplet analysis offers an interesting alternative way to study the effects of the nozzle turbulence.

Figure 5.10(a) shows the droplet radius $d_{v}=\sqrt[3]{6 V / \pi}$, where $V$ is the droplet volume. The blue line shows the difference in number of droplets between case 2 and case 0 , while the red line shows the difference between case 2 and case 1 (been case 2 the case with the largest number of broplets overall).In a first place, it is worth noting that, as already expressed in many works $[19,20]$, the droplets of a diameter smaller than two times the cell size should be neglected, as the VOF method is likely incapable to accurately describe the droplet of such a size. The number of droplets clearly decrease exponentially as the droplet diameter increase. It appears evidently that cases 1 and 2 generates a significant higher number of droplets, but no significant variation can be appreciated between this two cases. On the other hand, the difference between case 0 and cases 1 and 2 decrease almost exponentially. The difference between case 2 and 1 is almost negligible in respect to the total number of droplets in the 2 cases and it may be assumed that it is within the variability introduced by the random matrix generated for the synthetic boundary condition. Furthermore, is interesting to observe that case 1 and 2 also generates many liquid structures of significant size, showing that the instabilities generated by the synthetic boundary condition also allows the breakup of larger liquid structures (as it can be appreciated in Figure 5.9).

Figure 5.10(c) and 5.10(d) show respectively the Ohnesorge $(O h)$ and the Weber $(W e)$ number for the droplets. The Ohnesorge number is defined as:

$$
O h=\frac{\mu}{\sqrt{\rho \sigma d_{v}}}
$$

It is important to notice that, in this case, the droplet velocity used for the $W e$ number determination in equation 5.2 is the absolute velocity of the droplet, being difficult to apply the previous criteria for the relative velocity $u_{r}$ in the denser regions. Also, in this time average, there are no effects given by the tip vortices, therefore the faster droplets are likely to be located closer to the spray core. The higher number of droplet with a lower $W e$ number are representative of significant number of droplets with lower velocity and/or 
smaller size. These droplets are mostly the droplets that are stagnating in the peripheral area of the spray, where the spray cone angle is usually defined. As already said, for case 0 no spray axial breakup occur, therefore no spray cone angle can be defined. For this reason there are no droplets with higher $W e$ for case zero, meaning that the droplets detected are mostly in calm air regions and no new droplets (with higher kinetic energy) are formed in the time span where the analysis have been pursued. On the other hand, the $O h$ seems to behave in the same way for the three cases (as a consequence of a similar distribution for the droplet diameter) and highlights a general predominance of the viscous forces in respect of the surface tension forces.

Finally, Figure 5.10(b) shows the number of droplet and their radial distribution. Again, as for Figure 5.10(a) the blue and red lines are the difference in number of droplets as noted in the legend. For case 0 the number of droplet is decreasing faster as the radial position increase. This is a clear evidence that the droplets do not posses enough velocity to reach the domain borders, while case 1 and especially case 2 presents a high number of droplets at farther radius, implying a higher kinetic energy associated to each droplet. Figure 5.10(b) also allows to appreciate one of the biggest contribution introduced by the increment of the turbulence lengthscale. While the difference in the number of droplets between case 2 and 1 is relatively small at lower radius (less than one order of magnitude), it gets quite significant as the distance from the spray axis increase. This behaviour is quite significant as it is strictly related to the spray cone angle. In fact, experimental observations, made by means of Mie technique, identify the last region with a significant concentration of droplets as the region that defines the spray cone angle, that in this case would be significantly different for the three cases. As the spray cone angle observed in DNS simulations may be difficult to immediately compare with experimental data, these considerations will be useful guidelines for future studies.

\subsection{Observation on Gaussian behavior of the spray}

As already seen in Figure 5.7, no axial breakup would be possible without simulating an appropriate turbulent boundary condition at the nozzle outlet. This process is fundamental in breaking the liquid core continuity. Furthermore, the turbulence inlet boundary condition is responsible for the axial velocity drop, causing the radial velocity to be distributed as a gaussian profile instead of top-hat profile. 


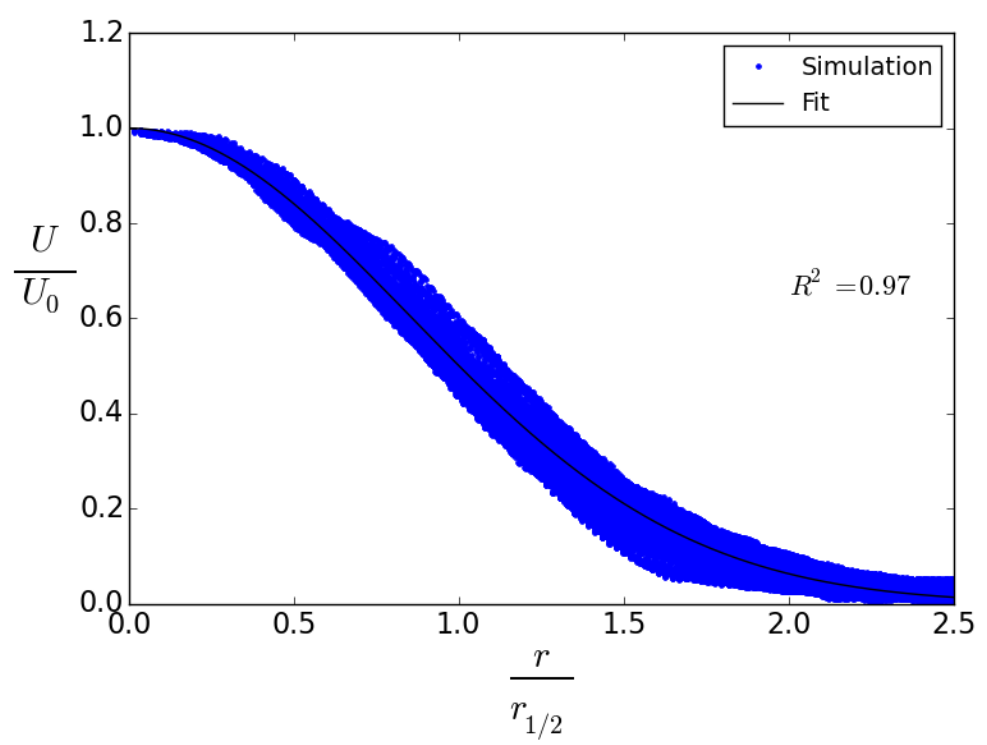

Figure 5.11: Self-similar profile obtained for case 1 at $x / D=22$

As proved in $[9,21]$, a spray displays a gaussian behavior after a certain penetration. In other words, the dimensionless axial velocity (made dimensionless by dividing the temporal-averaged component $U$ by the centerline velocity $\left.U_{0}\right)$ drops with the same behaviour along the dimensionless radius (made dimensionless by dividing the radius $r$ by the radial location at which the axial velocity drops by $50 \%$ in respect to the centerline velocity, $r_{1 / 2}$ ) at any axial position. More information about the axial velocity as a function of the density ratio between liquid and gas can be found at [22], that justify the applicability of the study in [21] to sprays. Figure 5.11 shows the gaussian profile obtained for case 1 at $x / D=22$. The blue dots are the velocity values for all the points in that section, while the black line is the data interpolation, according to the following correlation

$$
U(x, r)=U_{0}(x) \exp \left(-\alpha\left(\frac{r}{r_{1 / 2}}\right)\right)
$$

provided in [22], where $\alpha$ is the coefficient for the gaussian radial profile, explained in [22]. This behaviour is obviously kept for each section at $x / D>$ 22. The agreement between the simulation results and the expected behaviour can be assessed by the goodness of the fit. While the general behaviour is 
well captured, even better agreement is expected for higher wash-out time (time used by the fluid to get from the inlet to the outlet of the simulation domain), by increasing the simulation time. Due to the limitations in the domain size, dictated by the increasing computational costs, no other section can be displayed, because either to close to the outlet boundary, the nongaussian region or to the section displayed.

As this behavior emerges clearly for case 1 and 2, it is clear how an inlet turbulent boundary condition is essential in order to reproduce the real behavior of the spray. Furthermore, the characterization of $L$ and $I$, only achievable throughout simulation analysis, will be addressed in the next chapters in order to provide an improved comparability betweem the SBC and the MBC case.

\subsection{Main conclusions}

The analysis on the synthetic inlet boundary condition has provided many interesting considerations that have been used as drivers in designing the simulations presented in the next chapters. In a first place, the droplet distribution analysis highlights how, excluding case 0 , the trend is not dictated by the amount of turbulence generated within the nozzle. In fact, a higher value of $L$, for identical physical conditions (as the ones used here), means that the turbulence generated within the nozzle has larger scale separation (higher ratio between $L / \eta$ ), hence the larger $L$, the more turbulent energy is injected into the system. On the other hand, while the statistical distribution of the droplet generation seem to have its origins in the turbulence generated, typical features observable experimentally as external non-perturbed length, spray cone angle and axial mass concentration are heavily affected by $L$. Finally, case 0 seem to be completely unrepresentative of the physical problem, hence it will be avoided in future studies.

Some more practical remarks can also be made:

1. The transient analysis showed how the main vortex, the toroid one generated during the spray transient, may not be properly captured by the domain shape. It appears clearly in Figure 5.4 that some streamlines are almost tangent to the outflow boundary condition. While this is difficult to evaluate in the transient phase and no errors have been detected, it remains to be proven. On the other hand, the computing time required for such demanding simulations, as well as the wall-clock-time required for the post-processing, have dictated a more "safe" approach. Consequently, the domain in future works will be doubled in the radial 
directions $(y$ and $z)$. This increase the number of cells by a factor 4 , but also allow to avoid the analysis of the issue, which may requires a significant number of simulations just to characterize the issue. As the simulations presented in the next chapters have showed a more reliable behavior, this issue is considered solved.

2. The statistics are clearly insufficient for characterizing turbulence. This can be clearly observed by the dispersion of radial data in Figure 5.11. We define the number of wash-out as

$$
N_{w}=U_{i n j} T_{t o t} / l_{x}
$$

where $l_{x}$ is the length of the domain in the injection direction and $T_{t o t}$ is the total simulation time. In this case $N_{w} \approx 4$, which is obviously insufficient. Due to the high air density, it may be a safe assumption to consider that almost 2 wash-outs are used for the full advection of all major structures. In order to reach a safe number of $N_{w} \geq 10$ the total time has been increased by three times.

3. As already discussed, the increment of $L$ leads to an increment of turbulence level, hence more breakup occurs. Although extremely qualitative, this can be used as a "sanity-check" for the synthetic turbulent boundary conditions, aside from the considerations discussed above.

From a more operational standpoint, these simulations have proved to be of enormous size. The total size of the raw data reaches the $4 \mathrm{~TB}$ of data. As discussed already, the simulations that will be discussed in the following chapters presents 4 times more domain and 3 times more simulations time. In other words, replicating this study would cost around 48TB of storage data. This represents a significant issue, both financially and technically, as BigData algorithm should be employed. In order to reduce the data to process we chose two approaches:

- The data output format has been changed from ASCII to compress XML format, using the VTK library. This has the advantage of reducing the data storage (of about 70\%) and allow for parallel post-processing (e.g. within the Paraview environment).

- As the first 2 wash-outs are important just for the transient, a high output frequency is used to capture it. After the spray has fully penetrate, 
the sampling decrease. Furthermore, for the spectral analysis, only a plane (3 points in the injection direction) is output. This consideration has already been discussed in Chapter 3

\section{References}

[1] Lebas, R., Menard, T., Beau, P.A., Berlemont, A., and Demoulin, François-Xavier. "Numerical simulation of primary break-up and atomization: DNS and modelling study." In: International Journal of Multiphase Flow 35.3 (2009), pp. 247-260. DOI: 10 . 1016 / j . ijmultiphaseflow.2008.11.005.

[2] Klein, M., Sadiki, A., and Janicka, J. "A digital filter based generation of inflow data for spatially developing direct numerical or large eddy simulations". In: Journal of Computational Physics 186.2 (2003), pp. 652-665. DOI: 10.1016/S0021-9991 (03)00090-1.

[3] Hasslberger, Josef, Ketterl, Sebastian, Klein, Markus, and Chakraborty, Nilanjan. "Flow topologies in primary atomization of liquid jets: A direct numerical simulation analysis". In: Journal of Fluid Mechanics 859 (2019), pp. 819-838. DOI: 10.1017/jfm.2018.845.

[4] Benajes, Jesus, Salvador, Francisco Javier, Carreres, Marcos, and Jaramillo, David. "On the relation between the external structure and the internal characteristics in the near-nozzle field of diesel sprays". In: Proceedings of the Institution of Mechanical Engineers, Part D: Journal of Automobile Engineering 231.3 (2017), pp. 360-371. DOI: 10.1177/0954407016639464.

[5] Ghiji, M., Goldsworthy, L., Brandner, P. A., Garaniya, V., and Hield, P. "Numerical and experimental investigation of early stage diesel sprays". In: Fuel 175 (2016), pp. 274-286. DOI: 10.1016/j.fuel.2016.02.040.

[6] Hult, J. et al. "Interior flow and near-nozzle spray development in a marine-engine diesel fuel injector". In: Experiments in Fluids 57.4 (2016), p. 49. DOI: $10.1007 / \mathrm{s} 00348-016-2134-8$.

[7] Payri, Raul, Salvador, Javier, Gimeno, J., and De la Morena, J. “Analysis of Diesel Spray Atomization By Means of a Near-Nozzle Field Visualization Technique". In: Atomization and Sprays 21.9 (2012), pp. 753774. DOI: 10.1615/atomizspr. 2012004051. 
[8] Pickett, Lyle M. et al. "Comparison of Diesel Spray Combustion in Different High-Temperature, High-Pressure Facilities". In: SAE International Journal of Engines 3.2 (2010), pp. 156-181. DOI: 10.4271/201001-2106.

[9] Pope, Stephen B. Turbulent Flows. 2001. DOI: 10.1088/0957-0233/ 12/11/705.

[10] Bnà, S., Manservisi, S., Scardovelli, R., Yecko, P., and Zaleski, S. "Vofi - A library to initialize the volume fraction scalar field". In: Computer Physics Communications 200 (2016), pp. 291-299. DOI: 10.1016/j . cpc. 2015.10.026.

[11] Shinjo, J. and Umemura, Akira. "Simulation of liquid jet primary breakup: Dynamics of ligament and droplet formation". In: International Journal of Multiphase Flow 36.7 (2010), pp. 513-532. DOI: 10. 1016/j. ijmultiphaseflow. 2010.03.008.

[12] Shinjo, J., Xia, J., and Umemura, A. "Droplet/ligament modulation of local small-scale turbulence and scalar mixing in a dense fuel spray". In: Proceedings of the Combustion Institute 35.2 (2015), pp. 1595-1602. DOI: $10.1016 / j$.proci.2014.06.088.

[13] Ménard, T, Tanguy, S, and Berlemont, A. "Coupling level set/VOF/ghost fluid methods: Validation and application to $3 \mathrm{D}$ simulation of the primary break-up of a liquid jet". In: International Journal of Multiphase Flow 33.5 (2007), pp. 510-524. DOI: 10.1016/j.ijmultiphaseflow. 2006.11.001.

[14] Jarrahbashi, D., Sirignano, W. A., Popov, P. P., and Hussain, F. "Early spray development at high gas density: hole, ligament and bridge formations". In: Journal of Fluid Mechanics 792 (2016), pp. 186-231. DOI: 10.1017/jfm.2016.71.

[15] Jarrahbashi, D. and Sirignano, W. A. "Invited Article: Vorticity dynamics for transient high-pressure liquid injection". In: Physics of Fluids 26.10 (2014). DOI: 10.1063/1.4895781.

[16] Payri, Raul, Bracho, Gabriela, Marti-Aldaravi, Pedro, and Viera, Alberto. "Near Field Visualization of Diesel Spray for Different Nozzle Inclination Angles in Non-Vaporizing Conditions". In: Atomization and Sprays 27.3 (2017), pp. 251-267. DOI: 10.1615/atomizspr. 2017017949. 
[17] Vassallo, P. and Ashgriz, N. "Satellite Formation and Merging in Liquid Jet Breakup". In: Proceedings of the Royal Society A: Mathematical, Physical and Engineering Sciences 433.1888 (2006), pp. 269-286. DOI: 10.1098/rspa.1991.0047.

[18] Ashgriz, Nasser. Handbook of Atomization and Sprays. Springer Science \& Business Media, 2011. DOI: 10.1007/978-1-4419-7264-4.

[19] Shinjo, J. and Umemura, A. "Detailed simulation of primary atomization mechanisms in Diesel jet sprays (isolated identification of liquid jet tip effects)". In: Proceedings of the Combustion Institute 33.2 (2011), pp. 2089-2097. DOI: 10.1016/j.proci.2010.07.006.

[20] Ling, Yue, Fuster, Daniel, Zaleski, Stéphane, and Tryggvason, Grétar. "Spray formation in a quasiplanar gas-liquid mixing layer at moderate density ratios: A numerical closeup". In: Physical Review Fluids 2.1 (2017), p. 014005. DOI: 10.1103/PhysRevFluids.2.014005.

[21] Hussein J. Hussein, Steven P. Capps, and William K. George. "Velocity measurements in a high-Reynolds-number, momentum-conserving, axisymmetric, turbulent jet". In: Journal of Fluid Mechanics 001.258 (1994), pp. 31-75.

[22] Desantes, J. M., Salvador, F. J., López, J. J., and De La Morena, J. "Study of mass and momentum transfer in diesel sprays based on X-ray mass distribution measurements and on a theoretical derivation". In: Experiments in Fluids 50.2 (2011), pp. 233-246. DOI: $10.1007 /$ s00348010-0919-8. 



\section{Chapter 6}

\section{Analogies between multiphase and single-phase turbulence}

Before moving on in drawing significant physical analysis of the spray turbulent field, the hypothesis and the methodologies presented in Chapter 4 need to be discussed. In a first place, a discussion over the statistical convergence of turbulent variables will be performed, aiming at demonstrating that the total number of washouts used for the analysis is sufficient. While convergence of average fields, such as mean velocity is quite easy to achieve (see the discussion in Section 5.6) the pulsating values may be difficult to bring to convergence. For this reason, the statistical convergence of the PDF for both pulsating axial velocity $u$ and axial derivative of pulsating component $\partial_{x} u$ will be showed and discussed.

The pseudo-fluid hypothesis will be discussed in details, and its influence in computing the variables that are typical of turbulent flows (such as energy dissipation rate) will be highlighted. This hypothesis is an important foundation of the discussion that will be provided in the next chapter, and relies on some brutal and very effective simplifications of the flow features (such as surface location), hence its implications needs to be discussed.

Also, a few discussions over the conditions used for the simulation will be discussed. The implications of a low Reynolds number setup will be shown via a complete description of turbulent scales (from the largest to the smallest) and demonstrating how the turbulent spectra is indeed heavily affected by it. 
The following data and analysis will be performed for the simulation where the mapped boundary condition is used. The physical properties of this simulation and the domain design (dictated by the findings in Chapter 5) are presented in Tables 6.1 and 6.2 respectively.

\begin{tabular}{|c|c|c|c|c|c|c|c|}
\hline$R e_{b}$ & $\rho_{l}$ & $\rho_{g}$ & $\mu_{l}$ & $\mu_{g}$ & $\sigma$ & $\langle U\rangle$ & $D_{n}$ \\
\hline 5037 & 750 & 22.8 & $1.34 \cdot 10^{-3}$ & $1.85 \cdot 10^{-5}$ & $2.53 \cdot 10^{-2}$ & 100 & 90 \\
\hline- & $\mathrm{kg} / \mathrm{m}^{3}$ & $\mathrm{~kg} / \mathrm{m}^{3}$ & $\mathrm{~Pa} \cdot \mathrm{s}$ & $\mathrm{Pa} \cdot \mathrm{s}$ & $\mathrm{N} / \mathrm{m}$ & $\mathrm{m} / \mathrm{s}$ & $\mu \mathrm{m}$ \\
\hline
\end{tabular}

Table 6.1: Physical simulation properties for the case studied.

Again, the case simulated is dodecane for the Spray A configuration as for the study in Chapter 5 and the simulation physical properties are reported in Table 6.1 .

\begin{tabular}{|c|c|c|c|c|c|}
\hline$d x$ & $d t$ & $l_{x}$ & $l_{y, z}$ & $T_{t}$ & $T_{s s}$ \\
\hline 2.34 & 4.0 & 2.4 & 1.2 & 0.04 & 0.26 \\
\hline$\mu m$ & $n s$ & $m m$ & $m m$ & $m s$ & $m s$ \\
\hline
\end{tabular}

Table 6.2: Simulation setups.

Finally, a render of the simulation analyzed in this chapter can be found in Figure 6.1. Here, the VOF contour is colored by the local velocity magnitude, while the shadowed green plane display the region where the statistical analysis is performed (see Chapter 4)

\subsection{Statistics convergence and analysis.}

\subsubsection{Reasons for the post-process design}

As already discussed in Chapter 4, the main analysis for sprays in this thesis will be performed in a single plane in an axial position of $x / D_{n} \approx 25$. This choice was exclusively dictated by storage requirements imposed for performing statistical analysis of large data ensembles. In order to give an approximate idea, each plane in the simulation discussed here accounts for a domain of $3 \times 512 \times 512$ respectively in the $x, y, x$ directions. Each plane is saved for 60000 time-steps and it generates approximately $8 \mathrm{~TB}$ of raw data. Data compression can than be applied to take the storage requirement down of 


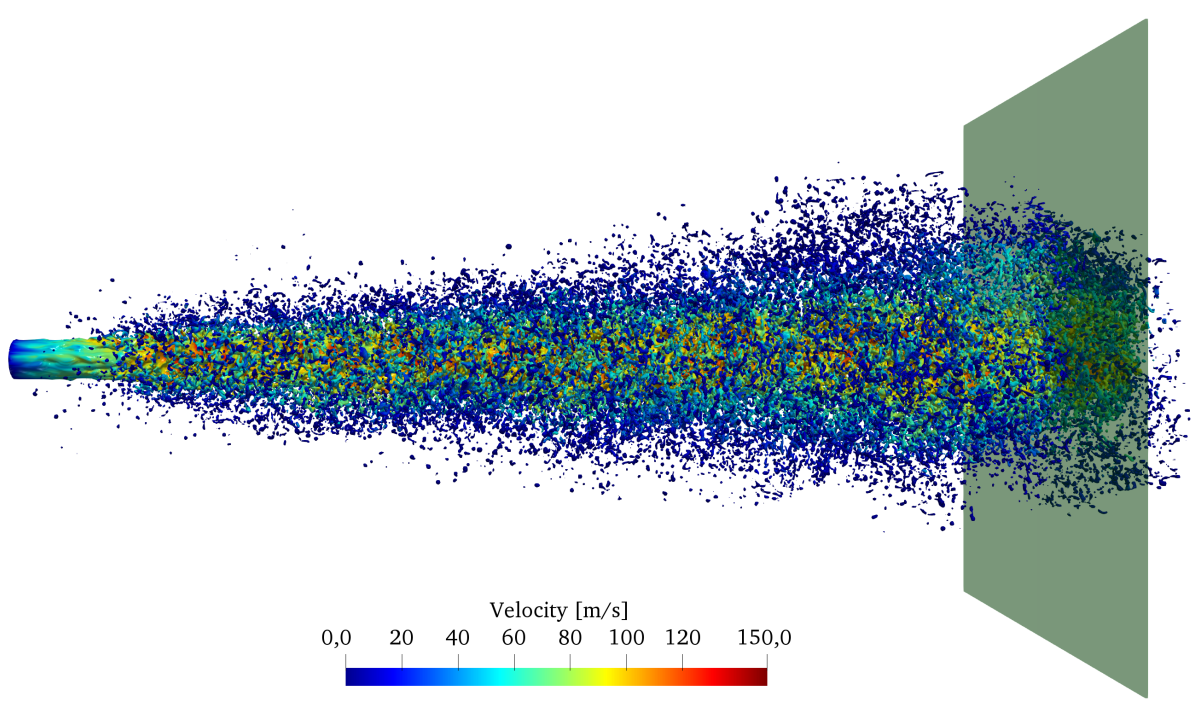

Figure 6.1: Render of the simulation described in Tables 6.1 and 6.2 and analyzed in this chapter. The VOF is contoured and colored by the local velocity. The shadowed green plane shows the position of the domain over which statistics have been analyzed.

about $70 \%$, reaching roughly $2.3 \mathrm{~TB}$ of compressed data. The post-processing can easily reach 10TB. This storage is required per each simulation, without accounting for the droplet analysis, which can easily reach a similar value. Furthermore, the turbulence statistics computation requires the development of ad-hoc parallel post-process environments/routines, which may require several days to run on a modern workstation (24 processors and 128GB of RAM). This enormous amount of data, explains why only one axial position has been analyzed and the significant amount of time required for both designing the post-process procedures and to run it.

A major objection to this procedure may be that these statistics can be performed on-the-fly (e.g. during the simulation runtime) as common practice in single-phase DNS and other theoretical studies that require exascale computing resources. While this is the correct way to proceed, also in the author opinion, it has some significant drawbacks. In a fist place, this procedure requires a well established and verified algorithm and a fully debugged code. In multiphase turbulence, there is no well established theory and many authors keep arguing whether the energy cascade needs to be similar to the 
one in singlephase flows, if it should be computed in the same way and what are the most important variables that dictate the behavior of the flow at both small and large scales. In this sense, what to obtain from the simulation is not known a-priori and therefore, a full output of a reduced region of the simulation is recommended.

\subsubsection{Statistics convergence}

As discussed previously, convergence of time-averaged variables is not per-se a proof of convergence of a statistical stationary process like turbulence is. In fact, Section 5.6 shows a self-similar velocity profile which almost reached statistical convergence, although the total average-time was about 3 washouts. The reason may be that the average performed here is not only temporal, but also spatial. As explained in Chapter 4, round sprays and jets have the common attribute of being axisymmetric, hence, statistically speaking many points at various azimuthal position and same radial position can be used to produce a smoother average. This procedure is robust from a physical standpoint and widely used in turbulence analysis of jets.

Figure 6.2(a) shows the convergence for the probability function of the velocity axial pulsating component, $u$, at a radial position $r=0.8 r_{1 / 2}$. This radial position has been chosen as far enough from the spray center. In this region, still significant atomization (secondary mostly) and breakup is occurring and the turbulent structures are, as will be discussed later, generally significantly smaller than the radius. The series of $N_{w}=10$ is the one used in general for all the statistics provided in this study. It is evident how the convergence is reached quite rapidly and already for $N_{w}=1.7$ the results are quite similar. The higher the number of washouts $N_{w}$, the smoother the statistics result. The Cartesian mesh that the simulation provides, is interpolated into a cylindrical mesh of 90 radial positions and 360 azimuthal positions. Surprisingly, similar results have been obtained for the $\mathrm{x}$-derivative of the $\mathrm{x}$-component of the velocity in Figure 6.2(b) at the same radial position, where a even better convergence is obtained. This behavior is quite unexpected as derivatives are usually noisy and even their average may result in noisy signals, while in Figure 6.2(b) a faster convergence than in Figure 6.2(a) is achieved. This aspect is surprising and will be investigated more in future research, although a few hypothesis can be made. In a first place, Figure 6.2(a) shows that velocity may have fluctuations that are comparable to the velocity average itself (that in this radial position may be around $60 \mathrm{~m} / \mathrm{s}$ ). This occurrences are capture even for low $N_{w}$, meaning that the phenomena has a significant statistical influence. On the other hand, the rapid decrement of the pseudo-Gaussian 


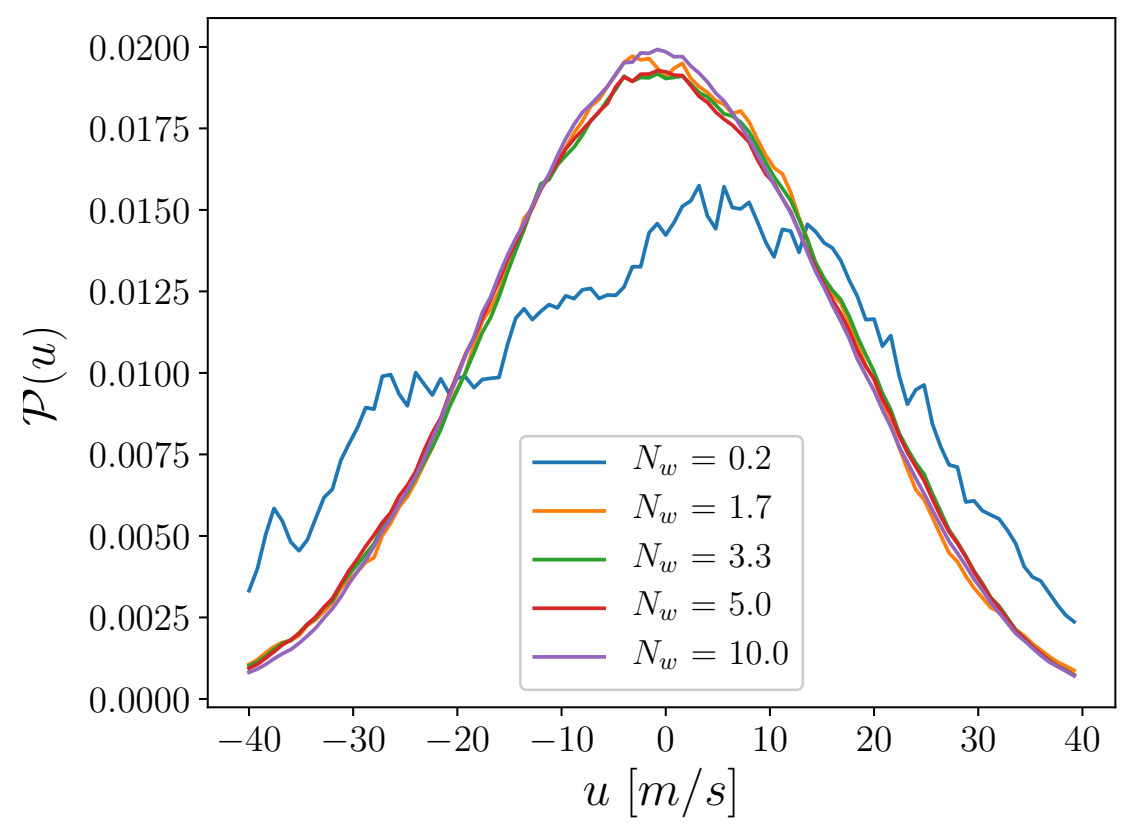

(a) Convergence of probability function for the axial velocity pulsating component.

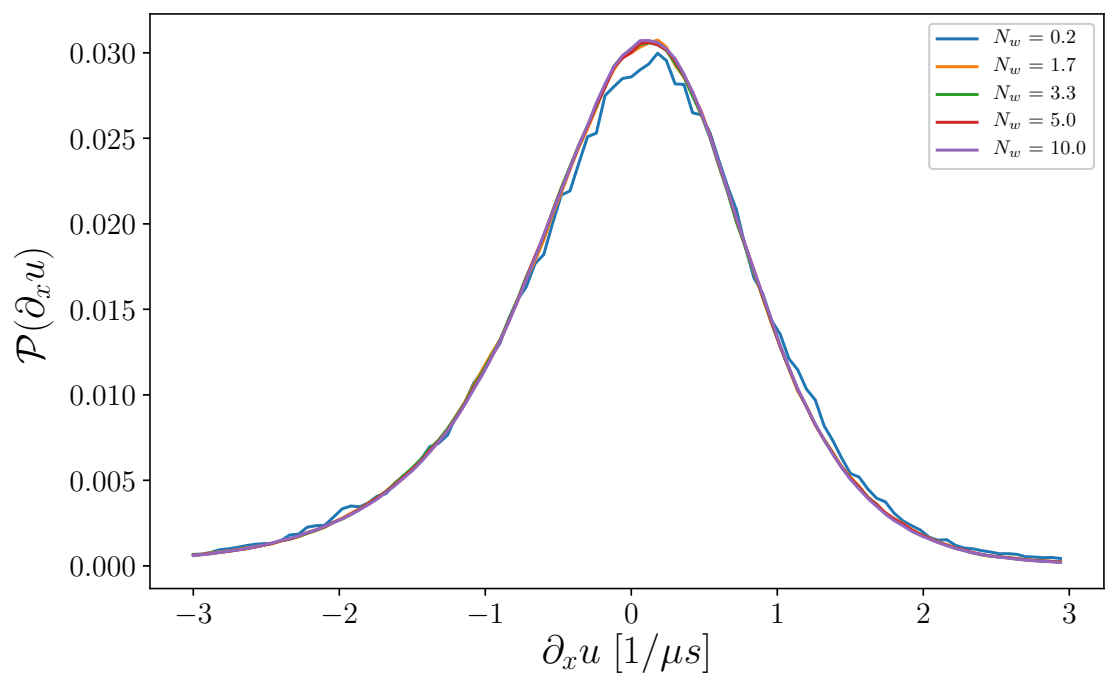

(b) Convergence of probability function statistics for $\mathrm{x}$-derivative the axial velocity pulsating component.

Figure 6.2: Convergence of the probability function statistics for axial velocity and its axial derivative. 
behavior of $\partial_{x} u$ decrease quite rapidly from its average peak, showing how turbulent structures are mostly transported axially and that their oscillations are quite small, as justified by the reduced turbulent field imposed by the low Reynolds number.

\section{Statistics analysis.}

A further analysis can be carried out on the probability function at different radial positions and it is showed in Figure 6.3. For both Figure 6.3(a) and Figure 6.3(b), the coloring goes from light gray to black as the radius increments from $r=0$ to $r=1.6 r_{1 / 2}$ with 40 line series. The pulsating axial velocity probability function, showed in Figure 6.3(a), displays a very interesting behavior, as radial positions closer to the spray centerlines are skewed towards the positive values while the farthest radius are skewed towards negative values. The transition is marked at $r=0.8 r_{1 / 2}$, showed in Figure 6.2(a), which is an almost perfect Gaussian. Although this behavior is quite rare, is not unique in literature and its validity has also been demonstrated mathematically by Jimenez [1]. Here the author argued that flows that have a spectra which is steeper than -1 in the inertial subrange (for 3D turbulence) do not necessarily display a Gaussian behavior in the pulsating velocity probability function. Future studies will address this issue in more details.

The axial derivative of the axial pulsating component of the velocity, showed in Figure 6.3(b), shows a behavior different to the one displayed by the velocity pulsating component in Figure 6.3(a). Here the behavior is still slightly skewed in a similar fashion, but the behavior is clearly more symmetric than for in Figure 6.3(a).

A deeper knowledge on the probability functions behavior may be reached by studying them at different axial positions, in order to prove if these patterns are maintained or if, by reaching a perfect self-similarity (with very low mass concentration in the spray centerline) the Gaussian profiles reach perfect symmetric. In that case, the velocity fluctuation may be related to the transition between the dense region and the dispersed region by analyzing the flow velocity probability also for other variables (e.g. mass concentration as a function of radius).

\subsection{The pseudo-fluid approach.}

As extensively described in Chapter 4, the classic theory of turbulence relies heavily on the description of material properties of the fluid. While in single 


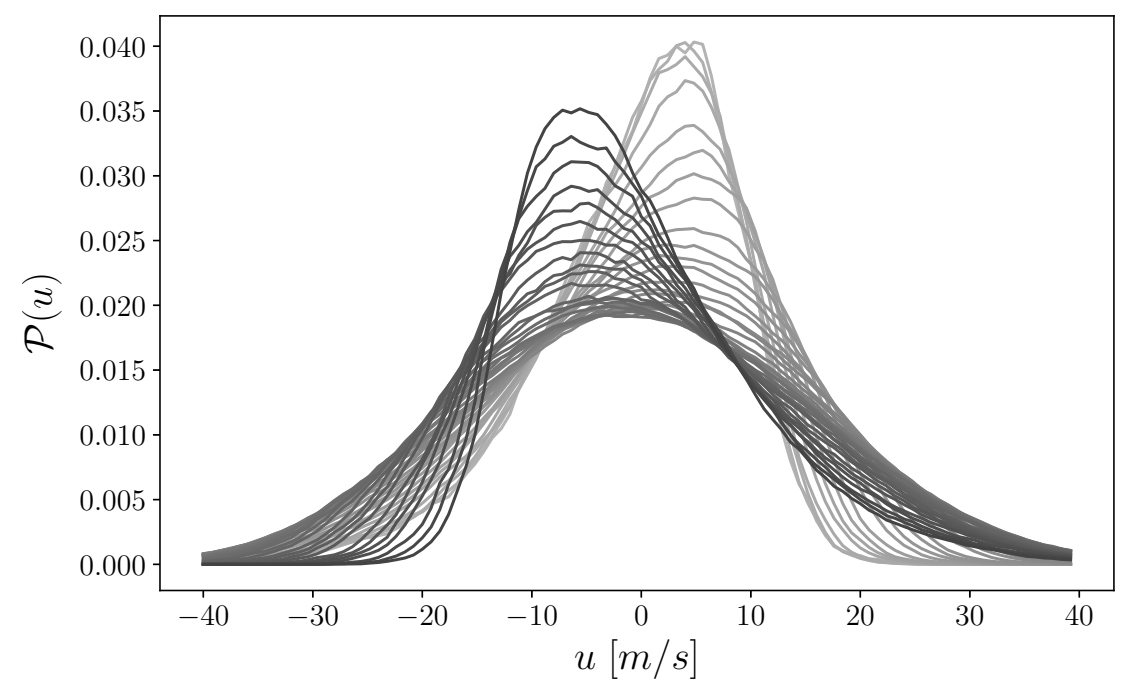

(a) Probability function for the axial velocity pulsating component at different radial position. The coloring goes from light grey to black as the radius increments. The series goes from $r=0$ to $r=1.6 r_{1 / 2}$ with 40 line series.

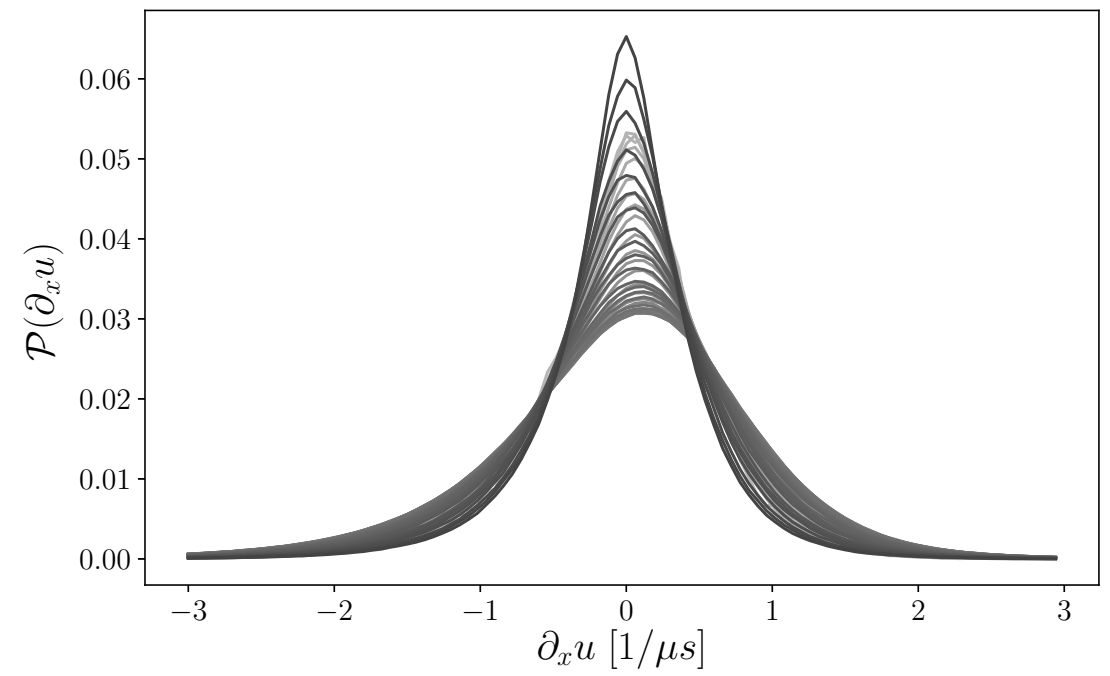

(b) Probability function for the axial derivative of the velocity pulsating component at different radial position. The coloring goes from light grey to black as the radius increments. The series goes from $r=0$ to $r=1.6 r_{1 / 2}$ with 40 line series.

Figure 6.3: Probability function as a function of radial position. 
phase flows these are easy to assess (in lack of compressibility or thermal gradients), in multiphase flows the issue is more substantial, throwing shades over the reliability and applicability of single phase turbulence in multiphase flows $[2,3]$. In fact, as a significant limit for the smallest scales of motion is imposed by the interface thickness, it is arguable that viscosity is still the only mechanism of dissipation [4].

The choice of using a pseudo-fluid approach is actually a way to address the multiphase turbulence and simplify an extremely complex scenario. In fact, the instantaneous description of the flow relies on its local properties $\rho(\mathbf{x}, t)$ and $\nu(\mathbf{x}, t)$, which are both function of time and position. The pseudofluid assumption in this case translates into accounting the time-dependence of these properties by substituting them by their time-average value in time. This may have some serious consequences in the determination of one-point statistics. For example, the actual definition of the average energy dissipation rate, in case of a phase change is:

$$
\epsilon=2\left\langle\nu s_{i j} s_{i j}\right\rangle=2\left(\langle\nu\rangle\left\langle s_{i j} s_{i j}\right\rangle+\left\langle\nu^{\prime} s_{i j} s_{i j}\right\rangle\right),
$$

where the viscosity has been decomposed into its average and pulsating component via a Reynolds decomposition $\nu=\langle\nu\rangle+\nu^{\prime}$. The pseudo fluid hypothesis consists in affirming that the second term on the last formulation is negligible, hence:

$$
\epsilon=2\left\langle\nu s_{i j} s_{i j}\right\rangle \approx 2\left(\langle\nu\rangle\left\langle s_{i j} s_{i j}\right\rangle .\right.
$$

In order to compute the average viscosity $\langle\nu\rangle$, the time-average volume fraction $\langle C\rangle$ is used and it is showed in Figure 6.4. This can be used instead of the Heaviside in Equation (2.2) to compute the time-average viscosity.

The result of the pseudo-fluid approximation is showed in Figure 6.5, where all the terms in Equation (6.1) are showed. As emerges from the analysis, the term $\left\langle\nu^{\prime} s_{i j} s_{i j}\right\rangle$ is negligible in respect to the average term $\langle\nu\rangle\left\langle s_{i j} s_{i j}\right\rangle$, validating the pseudo-fluid approach. Similar results have been found for variable viscosity fluid mixtures by Gauding et al. [5], where the fluctuating term also was negligible, despite the significant easier fashion with which mixing occurs in miscible fluids instead of multiphase flows.

An interesting consideration emerges from this analysis. Although the interface is a key factor in creating turbulence (e.g. via Kelvin-Helmholtz instability and droplet dispersion), its effect on the average analysis of turbulence is mostly negligible. This is a strong statement and needs further study 


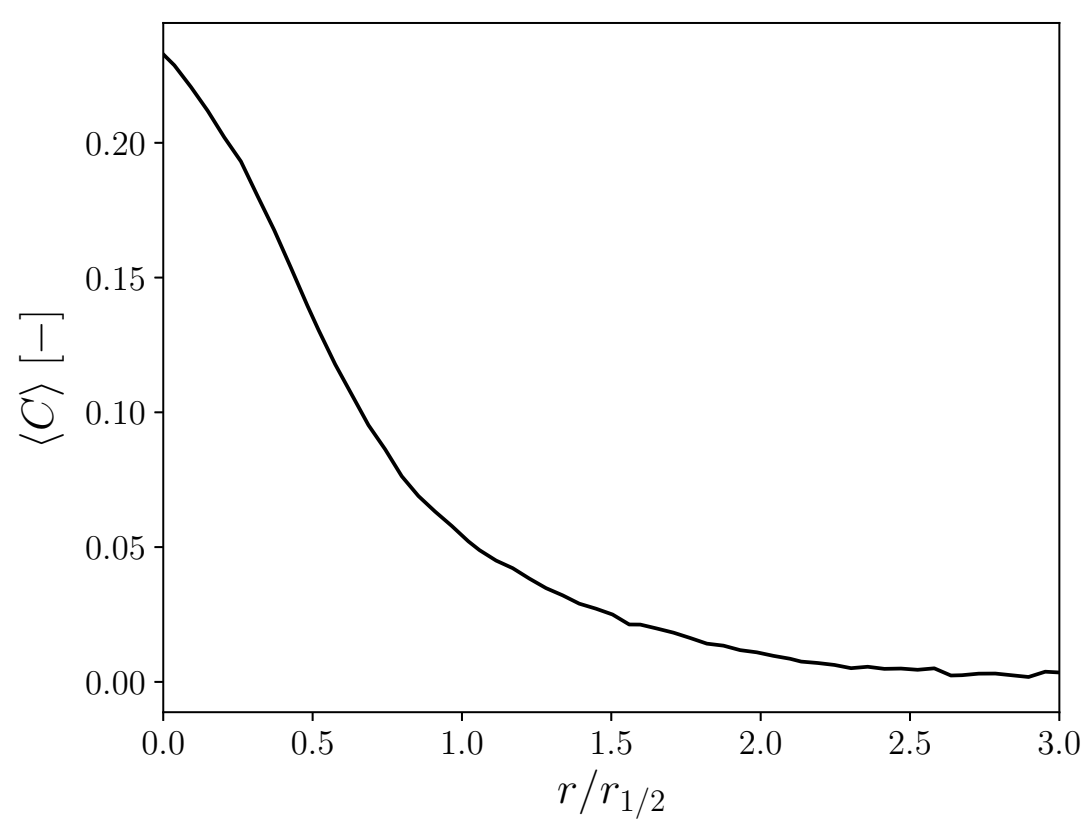

Figure 6.4: Time-average volume fraction as a function of the radial position.

and validation, although, if proved, could affect significantly the future studies of turbulence in multiphase flows. In fact, this hypothesis could also be a significant way to simplify the sub-grid modeling of turbulence in multiphase flows.

Accordingly to what discussed above, also the Kolmogorov scale can be computed and the result is showed in Figure 6.6. The Kolmogorov scale actually provides a very interesting insight on the flow behavior. In a first place, unlike the calculation of the Kolmogorov scale in particle-laden $[6,7]$ and droplet in isotropic turbulence [8], here the flow is strongly anisotropic, hence the local value of the Kolmogorov scale changes with the radial position. While this statement seem in contradiction with the academic explanation of this scale (usually described as the lengthscale characterizing the smallest eddies in a flow, hence uniquely defined by a single value) it actually opens up a newer interpretation of the scale in such a context. The smallest eddies are also characterized by a short timescale, hence they dissipate quite rapidly. It is therefore assumable that while they may get advected axially by the main flow, their radial displacement may be too slow to actually transmit the eddies generated in higher turbulent region to the outer diameters. If that 


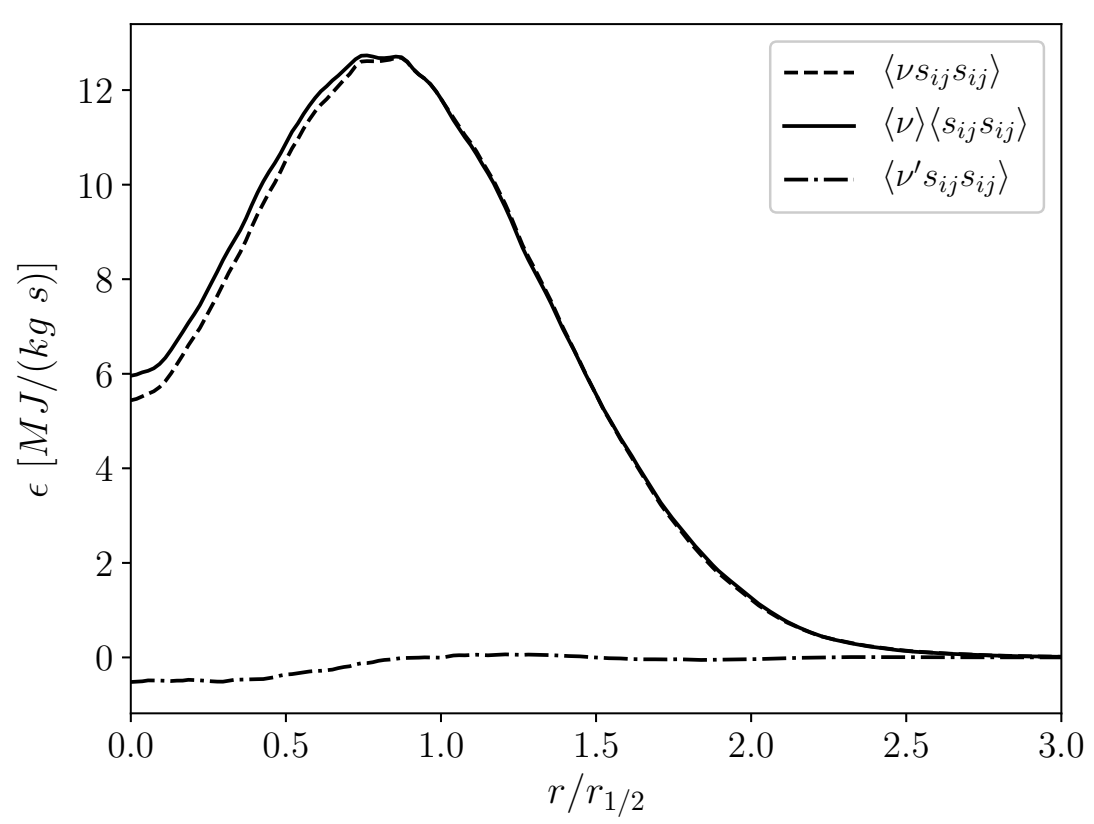

Figure 6.5: Average energy dissipation rate terms, as presented in Equation (6.1).

is the case, the information condensate in Figure 6.6 may be an excellent guideline for reduce the computational time required for these flows. In fact, as the mesh size may decrease rapidly with the radial position, the information over $\eta$ may provide a useful guide for algorithms like AMR, in order not to lose sensible informations over the turbulent structures.

\subsection{Autocorrelation and integral lengthscale.}

The first step in analyzing the flow spectra is computing the autocorrelation function, as discussed in Chapter 4. Also just in itself, the autocorrelation offers the chance of evaluating how the velocity keeps its self-relation, hence gives an estimation on whether a turbulent structure can be defined within a certain length. For example, an autocorrelation that does not reach zero is symptomatic of a turbulent structure that cannot be entirely defined within the length considered. Due to the radial approach that has been used in this thesis for the spectral analysis the autocorrelation at low radial distance from the spray centerline have a limited perimeter, hence they are likely to not 


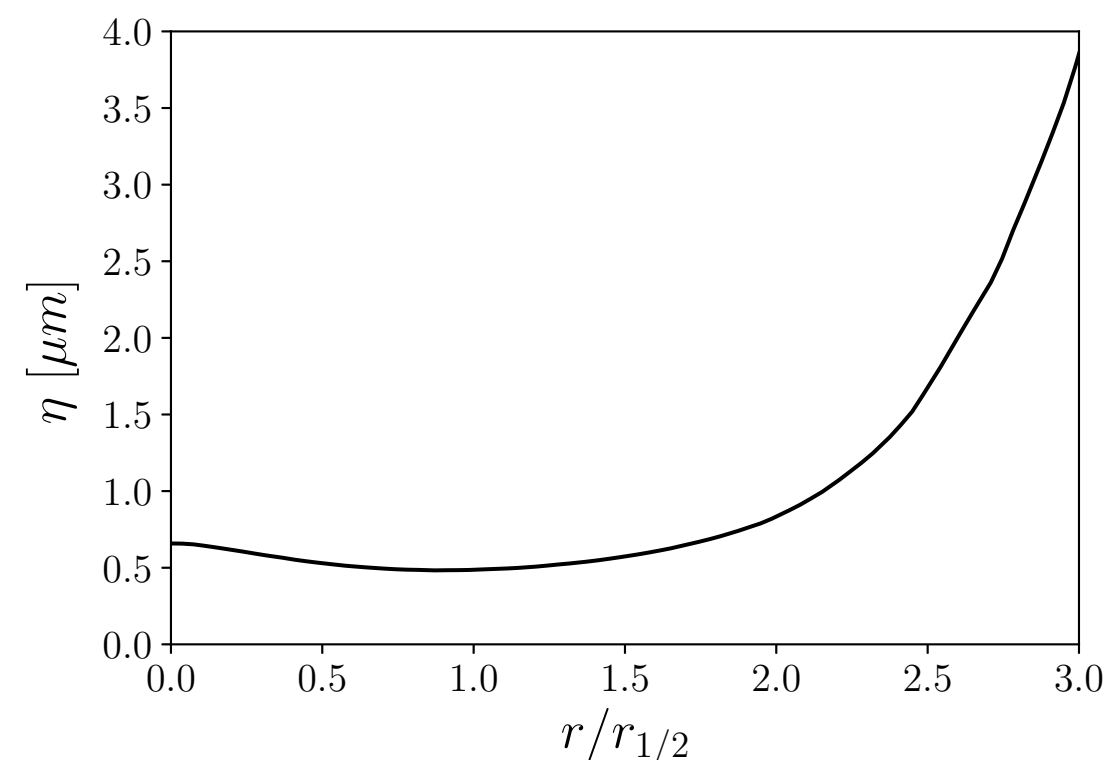

Figure 6.6: Kolmogorov scale as a function of the radial position.

reach zero, as some larger structures that goes through the spray centerline are indeed larger than their perimeter. This side-effects rapidly mitigates as the radial position increase and soon becomes secondary.

Figure 6.7 shows the autocorrelation function $f_{11}$ (described in Equation (3.13)) for the MBC case as a function of the dimensionless azimuthal coordinate $\xi$. In the legend each curve is described by its radial position $\left(r / r_{1 / 2}\right)$, its Reynolds-Taylor $R e_{\lambda}$ number and its Kolmogorov scale $\eta$. The radial positions showed have been picked to avoid the above mentioned effect (which will be showed later in Chapter 7). All the autocorrelation picked show a rapid decay, meaning that the turbulent structures have a shape that is significantly smaller than the radial perimeter over which the autocorrelation is computed. Also, the farther from the spray axis, the more rapidly the autocorrelation decays and this behavior can be explained by the fact that, as stated above, the perimeter over which the $f_{11}$ is computed increase with the radius.

Another interesting consideration can be made by computing the integral lengthscale $L_{11}$ (as presented in Equation (3.12)). The result, as a function of the dimensionless radial position $\left(r / r_{1 / 2}\right)$ is showed in Figure 6.8. The overall behavior is quite similar to the Kolmogorov scale in Figure 6.6, showing a scale separation $\left(L_{11} / \eta\right)$ within $1-2$ orders of magnitude. It can be noted 


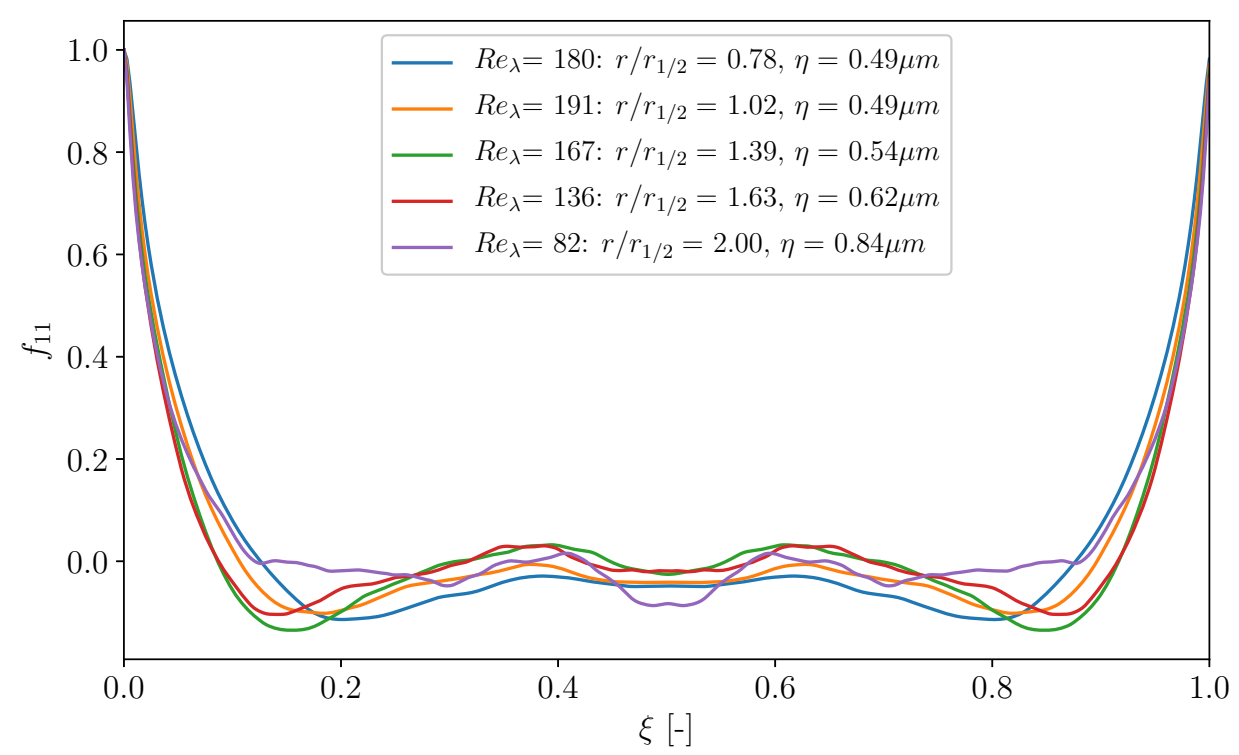

Figure 6.7: Autocorrelation function $f_{11}$ for the $M B C$ case as a function of the dimensionless azimuthal coordinate $\xi$. In the legend each curve is described by its radial position $\left(r / r_{1 / 2}\right)$, its Reynolds-Taylor $R e_{\lambda}$ number and its Kolmogorov scale $\eta$.

that, within the mixing region, the integral lengthscale is of the order of the nozzle radius, while progress quite rapidly to almost twice the diameter as the radius increases. This information also provides a interesting insight on how the Kolmogorov scale is computed in order to justify, a priori, the mesh size in DNS simulations. In fact, in many works [9-11] the determination of the Kolmogorov scale (hence the mesh size) relies on the parameters provided for the turbulence developed within the nozzle flow (e.g. $L$ and $I$ in the SBC case described in Chapter 3). The difference between this value of $L_{11}$ and the one found in Section 3.2 shows clearly why this practice should not be, in the author opinion, pursued. On the other hand, this approach is reasonable in lack of data to compute $\eta$ otherwise.

\subsection{Kolmogorov spectra scaling.}

Using the autocorrelation data (in their non dimensionless form) showed in Figure 6.7, the one dimensional energy spectra of the flow can be computed. Figure 6.9 shows the energy spectra, made dimensionless using the 


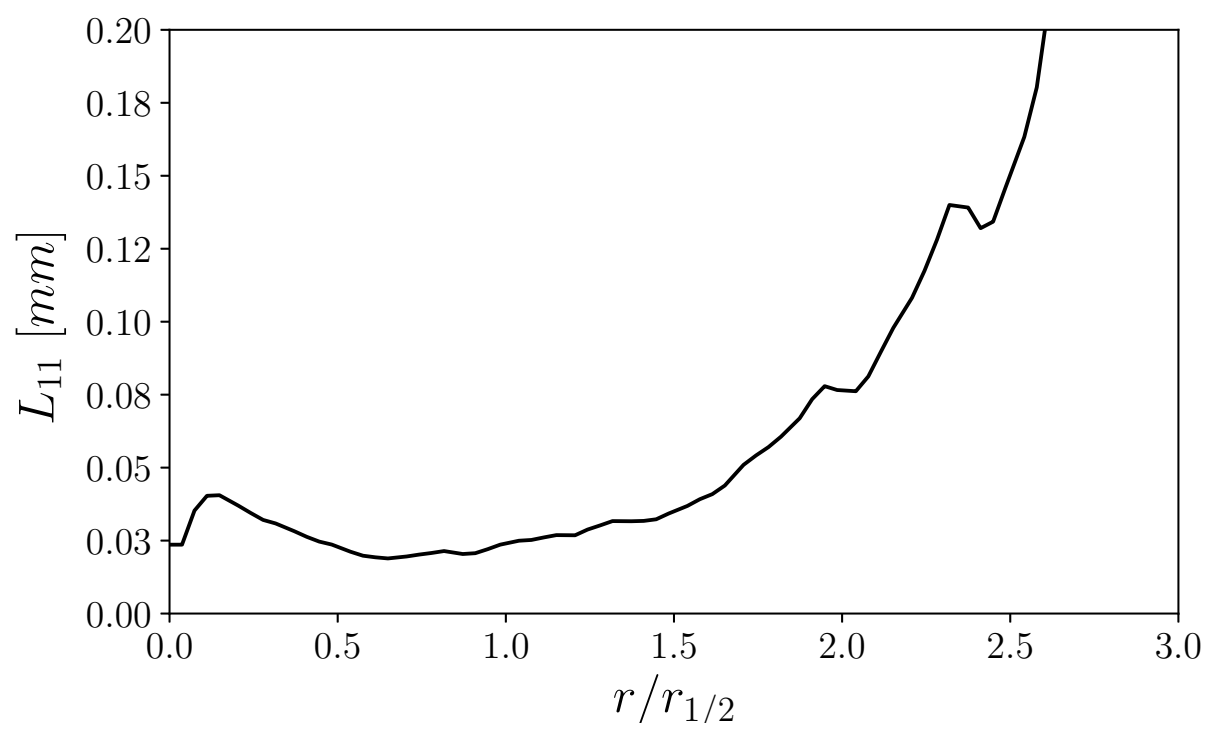

Figure 6.8: Integral lengthscale $L_{11}$ as a function of the dimensionless radial position $\left(r / r_{1 / 2}\right)$.

Kolmogorov scaling $[13,16]$. Each value of $\eta, \nu$ and $\epsilon$ has been taken at the exact radial position where the respective autocorrelation is computed from the data displayed in previous section in this chapter. The Kolmogorov scaling representation of the one-dimensional spectra presents many advantages. In a first place, it enables the comparison of different spectra computed in various ways, from both experimental and numerical analysis. In Figure 6.9, the comparison with data from Uberoi and Freymuth [12], Veeravallif [13], Harris et al. [14], and Comte-Bellot and Corrsin [15] (as reported in [13, 16]) is showed. The spectra for different radius collapse for $\kappa \eta>0.1$ as discussed in [16]. This behavior is typical of flows that verify the first similarity hypothesis (see Section 4.1.1), for which it can be proved (see $[16,17]$ ) that the one dimensional spectra is a function of $\kappa \eta$. Furthermore, thanks to the Kolmogorov scaling, some interesting considerations over the pseudo-fluid approach can be made. In fact, the curve collapsing shows that the turbulence behavior, although changes significantly with the radial position, maintains a behavior coherent with the Kolmogorov first similarity hypothesis. Furthermore, this also implies that the velocity spectra is not affected by the atomization process. In fact, Figure 6.4 shows that for $0 \leq r / r_{1 / 2} \leq 2$ there is still a quite significant atomization activity, therefore the droplet and breakup should affect the spectra. Instead, this data shows that it is likely that the breakup is actu- 


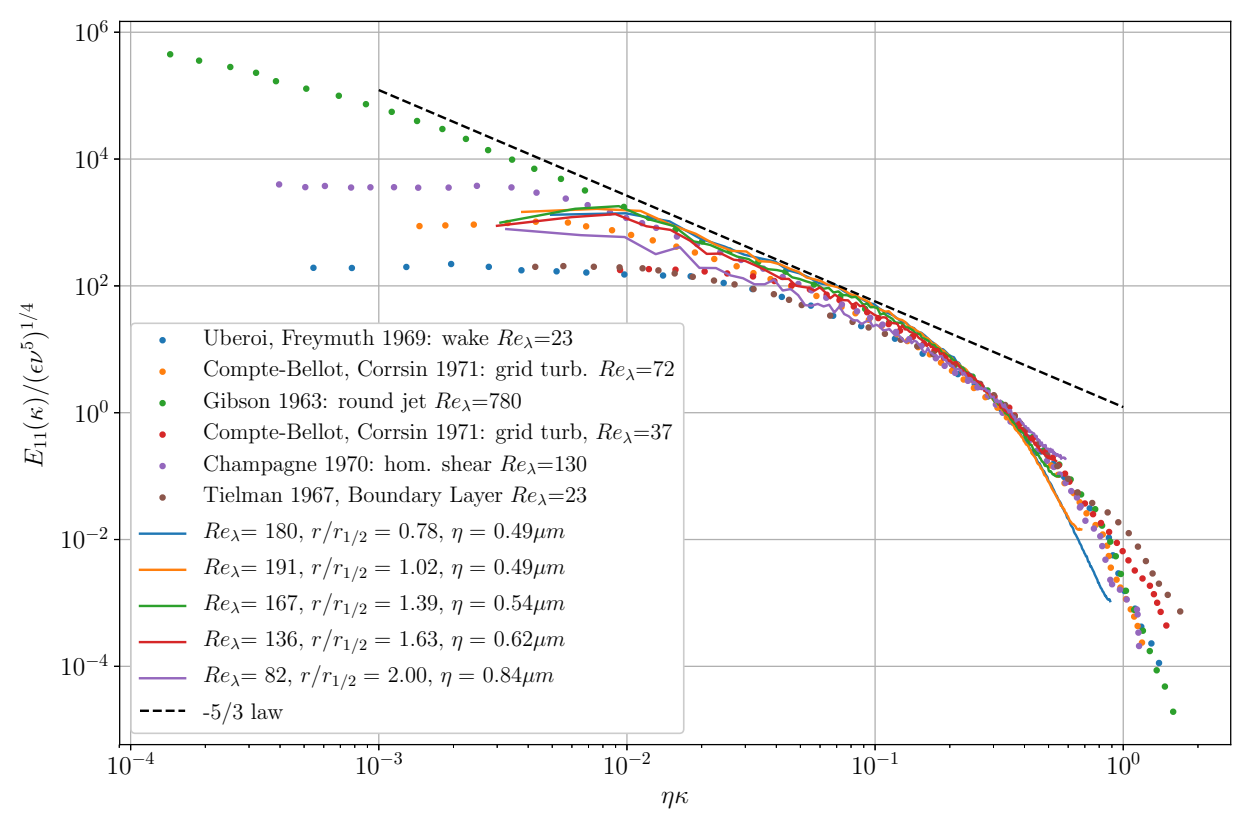

Figure 6.9: One dimensional energy spectra showed using the Kolmogorov scaling. Each radius is made dimensionless using its respective Kolmogorov scale in Figure 6.6 and the respective $\epsilon$ (Figure 6.5) and $\nu$. The data are from Uberoi and Freymuth [12], Veeravallif [13], Harris et al. [14], and ComteBellot and Corrsin [15].

ally a mechanism for increasing the turbulence level (hence $R e_{\lambda}$ ), therefore no significant disruption of the spectra in the transition between the inertial subrange and the dissipation range is cause, explaining the curves collapse for $\kappa \eta \approx 0.1$.

On the other hand, it is important to notice that while the comments above are valid regarding the level of turbulence (as confirmed by the findings of Uberoi and Freymuth [12]) this case shows a limited turbulence behavior, as the $-5 / 3$ law of the inertial subrange actually applies only for one order of magnitude on the wavelength axis, symptom of a reduced scale separation. This considerations can be extended to all the cases showed in this thesis, which are performed at the same $R e_{b}$. Still, this context provides an excellent test-case to prove the method that has been used in analyzing the spray and provide a significant insight on the spray turbulent behavior. While the scale separation reported here is not in line with state of the art single-phase flow turbulence analysis, is in line with state of the art studies of multiphase flows. 
Furthermore, the scale separation is still sufficient to assess a inertial subrange, hence turbulence is indeed developed to a level sufficiently high to appreciate the energy generation range, the inertial subrange and the dissipation range.

\subsection{Conclusions.}

The study performed in this chapter has various implications in the analysis of sprays and their physical interpretation. In a first place, it proves that the pseudo-fluid approximation for the study of turbulence can be used as demonstrated by Figures 6.5 and 6.9. Nevertheless, it may be argued that this may be an effect of the model used. In fact, as pointed out in literature (e.g. [10]) this issue may relate to the validity of the governing equations used (especially the color function advection), but it is difficult to asses. It can be argued that the result of this simulation and, in general, of simulations in literature using similar methods do not points toward a ill-posed problem from a physical standpoint. Therefore, the pseudo-fluid method allows for comparison with existent data from single-phase turbulent flows and gives room to possible comparisons, previously not available for multiphase flows in general.

Interestingly, while many variables seem to have a smooth behavior, the autocorrelation and the integral length scale show a certain roughness in Figures 6.7 and 6.8. This may be given by an insufficient ensemble time to perform the average of stationary stable sprays, although it seems that other variables showed do not point in towards that conclusion.

A strong skewness has been noted in the fluctuating velocity PDF in Figure 6.3(a), which needs to be addressed in further studies. This phenomena may be caused by a non developed spray (given by the dense region), an air-entrainment behavior of the spray or by the atomization process.

Finally, an interesting study suggested by these analysis may be the comparison of a single-phase jet in the same injection condition as the one presented here. This study would allow to analyze how the atomization affect the local level of turbulence $\left(R e_{\lambda}\right)$ and the spectral behavior, giving room for stronger conclusions on the pseudo-fluid method and the effects of atomization on turbulence and vice-versa.

\section{References}

[1] Jimenez, Javier. "Turbulent velocity fluctuations need not be Gaussian". In: Journal of Fluid Mechanics 376.-1 (2000), pp. 139-147. 
[2] Duret, B. et al. "DNS analysis of turbulent mixing in two-phase flows". In: International Journal of Multiphase Flow 40 (2012), pp. 93-105. DOI: 10.1016/j.ijmultiphaseflow.2011.11.014.

[3] Ling, Y., Fuster, D., Tryggvason, G., and Zaleski, S. "A two-phase mixing layer between parallel gas and liquid streams: Multiphase turbulence statistics and influence of interfacial instability". In: Journal of Fluid Mechanics 859 (2019), pp. 268-307. DOI: 10.1017/jfm. 2018. 825.

[4] Pitsch, H. and Desjardins, Olivier. "Detailed Numerical Investigation of Turbulent Atomization of Liquid Jets". In: Atomization and Sprays 20.4 (2010), pp. 311-336. DOI: 10.1615/atomizspr.v20.i4. 40.

[5] Gauding, M., Danaila, L., and Varea, E. "One-point and two-point statistics of homogeneous isotropic decaying turbulence with variable viscosity". In: International Journal of Heat and Fluid Flow 72.May (2018), pp. 143-150. DOI: 10.1016/j .ijheatfluidflow. 2018.05. 013.

[6] Park, George Ilhwan, Bassenne, Maxime, Urzay, Javier, and Moin, Parviz. "A simple dynamic subgrid-scale model for les of particle-laden turbulence". In: Physical Review Fluids 2.4 (2017), pp. 1-20. DOI: 10. 1103/PhysRevFluids . 2.044301.

[7] Bassenne, Maxime, Esmaily, Mahdi, Livescu, Daniel, Moin, Parviz, and Urzay, Javier. "A dynamic spectrally enriched subgrid-scale model for preferential concentration in particle-laden turbulence". In: International Journal of Multiphase Flow 116 (2019), pp. 270-280. DOI: 10. 1016/j. ijmultiphaseflow . 2019.04.025.

[8] Elghobashi, Said. "Direct Numerical Simulation of Turbulent Flows Laden with Droplets or Bubbles". In: Annual Review of Fluid Mechanics 51.1 (2018), pp. 217-244. DOI: 10.1146/annurev-fluid-010518040401.

[9] Lebas, R., Menard, T., Beau, P.A., Berlemont, A., and Demoulin, François-Xavier. "Numerical simulation of primary break-up and atomization: DNS and modelling study." In: International Journal of Multiphase Flow 35.3 (2009), pp. 247-260. DOI: 10 . 1016 / j . ijmultiphaseflow.2008.11.005.

[10] Gorokhovski, Mikhael and Herrmann, Marcus. "Modeling Primary Atomization". In: Annual Review of Fluid Mechanics 40.1 (2008), pp. 343366. DOI: 10.1146/annurev.fluid.40.111406.102200. 
[11] Hasslberger, Josef, Ketterl, Sebastian, Klein, Markus, and Chakraborty, Nilanjan. "Flow topologies in primary atomization of liquid jets: A direct numerical simulation analysis". In: Journal of Fluid Mechanics 859 (2019), pp. 819-838. DOI: 10.1017/jfm.2018.845.

[12] Uberoi, Mahinder S. and Freymuth, Peter. "Turbulent energy balance and spectra of the axisymmetric wake". In: Physics of Fluids 13.9 (1970), pp. 2205-2210. DOI: 10.1063/1.1693225.

[13] Veeravallif, Srtntvas V. "Local Isotropy in Turbulent Boundary Layers at High Reynolds Number". In: Journal of Fluid Mechanics 268.-1 (1994), pp. 333-372. DOI: 10.1017/S0022112094001370.

[14] Harris, V. G., Graham, J. A.H., and Corrsin, S. "Further experiments in nearly homogeneous turbulent shear flow". In: Journal of Fluid Mechanics 81.4 (1977), pp. 657-687. DOI: 10.1017/S0022112077002286.

[15] Comte-Bellot, Geneviève and Corrsin, Stanley. "Simple Eulerian time correlation of full-and narrow-band velocity signals in grid-generated, âisotropic' turbulence". In: Journal of Fluid Mechanics 48.2 (1971), pp. 273-337. DOI: 10.1017/S0022112071001599.

[16] Pope, Stephen B. Turbulent Flows. 2001. DOI: 10.1088/0957-0233/ 12/11/705.

[17] Cotton, William R., Bryan, George, and Heever, Susan C. van den. Turbulence. Vol. 99. C. Cambridge University Press, 2011, pp. 53-86. DOI: $10.1016 / \mathrm{S} 0074-6142(10) 09909-2$. 



\section{Chapter 7}

\section{Effects of isotropic and anisotropic turbulent structures on primary atomization}

The analysis performed in Chapter 5 highlighted how the amount of turbulence (in terms of the integral length scale $L$ ) developed into the nozzle may significantly affect the atomization process from a quantitative standpoint, while similar patterns in case 1 and 2 have been noticed as far as droplet statistics. On one hand, this "robustness" of droplet statistics may be important for fundamental statistical analysis of atomization, as the atomizations patterns seems to develop similarly regardless of the amount of perturbation developed into the nozzle (likely, as far as this variation are within physical values). On the other hand, comparison with experiments may be extremely challenging, as features like the external non-perturbed length and the spray cone angle are significantly different in the two cases. In this sense, if a DNS simulation should be compared with experiments, an ad-hoc inflow boundary condition should be generated in order to capture the actual dynamic of the flow. This raise further questions on the reliability of the nozzle flow simulations, as experiments providing data for turbulence profiles may be unfeasible.

In this context, the only nozzle with a significant experimental validation and a well established (although non conclusive) turbulence description is the 
pipe flow. This flow is quite representative of many cylindrical nozzles, but the actual length of the duct is often insufficient for a fully developed turbulent profile. Because of these uncertainties, many DNS simulations are still performed using the synthetic inflow boundary condition described in Section 3.1. The SBC on the other hand should have another important feature that should be handled with careful. In lack of data for the $a$ matrix (Equation (3.3)), the turbulence profile is often used in its version based on the homogeneous turbulence, hence generating homogeneous structures. This is quite a heavy hypothesis at large scale as strong anisotropy has widely been observed in wall flows [1]. In general, the nozzle flows are strongly anisotropic, hence the hypothesis may be heavily influencing the development of the spray, especially in the dense region, where the discussion in Chapter 5 proved that the irregularities on the spray core are responsible for the breakup of liquid structures. For this reason, the mapped boundary condition (hereafter MBC) described in Section 3.2 is compared with SBC. In order to improve the comparison between the two cases, the parameters $I$ and $L$ of the SBC have been calibrated as in Section 3.2.2.

On the other hand, the considerations for the domain design discussed in Section 5.7 have been accounted for, hence the new domain designed is described in Table 6.2. Here, $l_{x, y, z}$ indicate the domain size in each of the respective direction, $d x$ is the mesh size (spatially uniform due to Cartesian cubic cells), $d t$ is the timestep. As discussed in Section 5.7, transient information is of limited utility for significant statistics (at least for drawing significant physical conclusions). For this reasons, the statistical analysis of the spray has been performed for $T_{s s}$ time (statistically stationary time interval) after the spray has fully penetrated the domain axially (which is, after $T_{t}$ time). Obviously, $T_{t o t}=T_{t}+T_{s s}$ is the total time of simulation.

\subsection{Mean fields analysis using one-point statistics}

A first indication on the differences between the SBC and MBC can be obtained by analyzing the averaged fields. Figures 7.1 and 7.2 present the main and, perhaps, most important and documented quantities that can be obtained by performing a time-average operation (here, the operator \langle\rangle indicates the time-average) over the time span $T_{S S}$ on a plane located at $x / D=25$. A first important consideration should be made on the values of the centerline velocity $U_{c}$ (evaluated where the profile is calculated) and the radial position where the velocity have decayed by half of its centerline value, $r_{1 / 2}$. As this parameters are widely used in literature to compare jets and sprays at 
different injection conditions, here a special care should be devoted to their numerical value, as they are quite revealing on how the axial inlet velocity spreads radially. In fact, the MBC case opens up faster than the SBC cases, therefore showing a lower axial velocity $\left(U_{c, M B C} / U_{c, S B C}=0.95\right)$ and a higher $r_{1 / 2}\left(r_{1 / 2, M B C} / r_{1 / 2, S B C}=1.16\right)$. Furthermore, while both simulations are performed with the same mean velocity inlet profile, the MBC centerline axial velocity has overall decayed by a $20 \%$ (in comparison to its inlet value) while SBC roughly by a $15 \%$.

Despite the differences in the values of $U_{c}$ and $r_{1 / 2}$, Figure 7.1(a) displays a remarkable similarity in the Gaussian distribution of the velocity for the two cases, also when compared against the results obtained in [2] for a round jet. On the other hand, interesting differences can be observed in Figure 7.1(b). Here the comparison with [2] is avoided for clarity, but it is quite evident that although the overall behavior is preserved, the radial component is not yet as significant as reported in literature and this deserve to be discussed further. In a first place, it is evident that $U_{r}$ positive values are significantly lower than reported in literature, where $\max \left(\left\langle U_{r}\right\rangle / U_{c}\right)$ is 0.017 and it is reached at $r / r_{1 / 2}=0.6$. While a similar radial position for the maximum is showed for the $\mathrm{MBC}$ case, the lower values of the radial velocity suggests that the spray for both cases is still in its Near-Field region. Furthermore, in both cases the tendency of air entrainment is predominant over the spray spreading (the module of the absolute minimum is higher than the absolute maximum), which reinforce the previous hypothesis. Consistently with what has been observed in the $U_{c}, r_{1 / 2}$ discussion, the MBC case tends to have a wider radial spreading than the SBC case.

The velocity fluctuations are showed in Figure 7.2(a). Again, the inconsistency against literature data support the hypothesis that the spray self similarity is not yet fully developed. The maximum values for both the axial velocity fluctuations $\left\langle u^{2}\right\rangle$ and radial velocity fluctuations $\left\langle u_{r}^{2}\right\rangle$ are about $25 \%$ of values reported in literature. For $r_{1 / 2}<1$ the curves slope is still strongly positive, underlining how the influence of the liquid core is still predominant in the mean flow.

Of particular interest in this analysis is the averaged Heaviside function $\langle C\rangle$ (also considerable as a volume fraction) showed in Figure 7.2(b). For the reasons explained in Chapter 3, is of central importance in determining $\eta$, therefore for analyzing the turbulence behavior of the fluid. The MBC case presents a lower axial value of $\langle C\rangle$ which suggests a higher atomization. This assumption is consistent with the higher radial spreading of the MBC case in respect to the SBC. Integrating the value of $\langle C\rangle$ over the radius we can obtain 


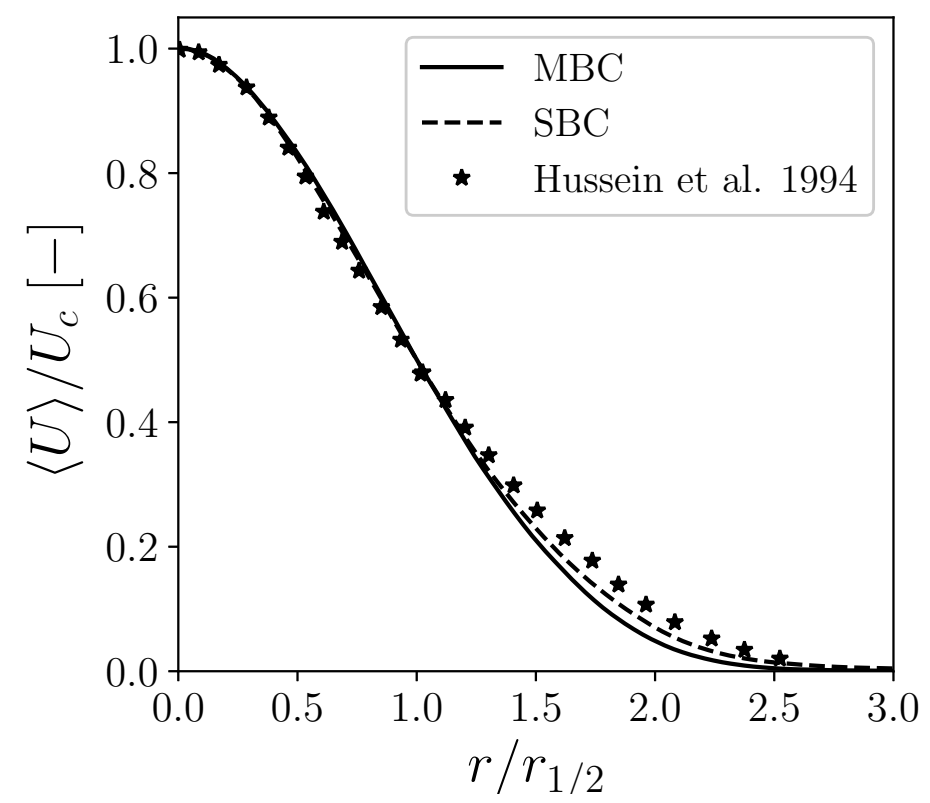

(a) Radial profile for the mean axial velocity component.The results are compared against experimental data from [2].

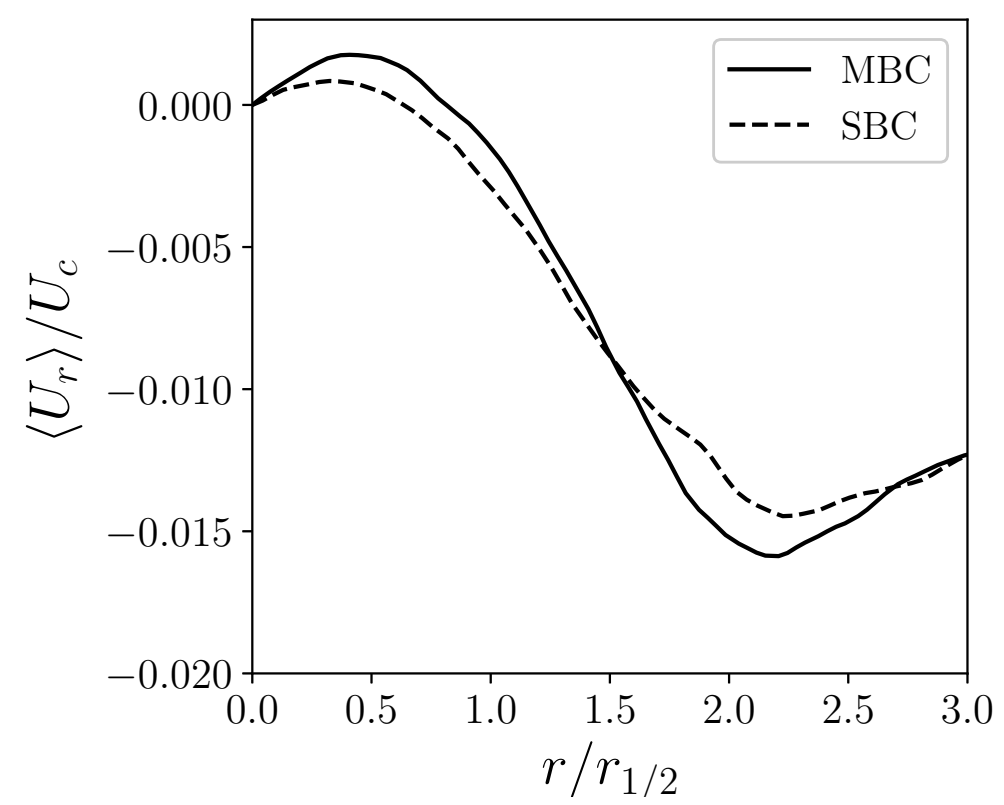

(b) Radial profile for the mean radial velocity component.

Figure 7.1: Time averaged fields. 


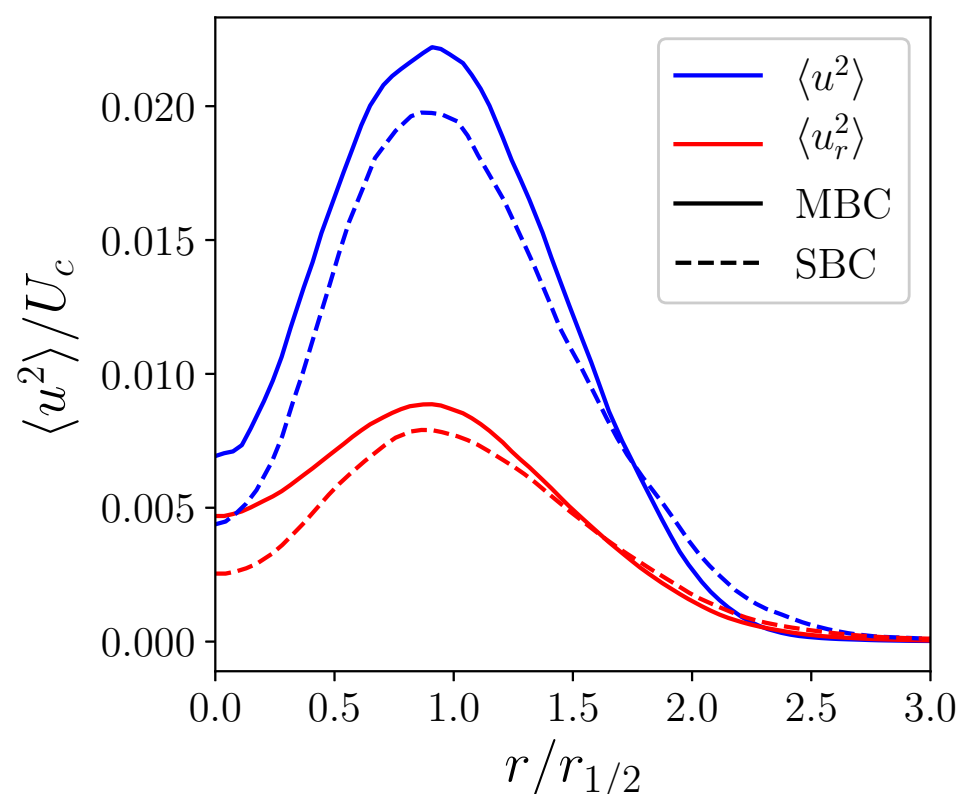

(a) Radial profile for the velocity fluctuating components. Line color represent the component (axial or radial) while the linestyle differentiate the cases.

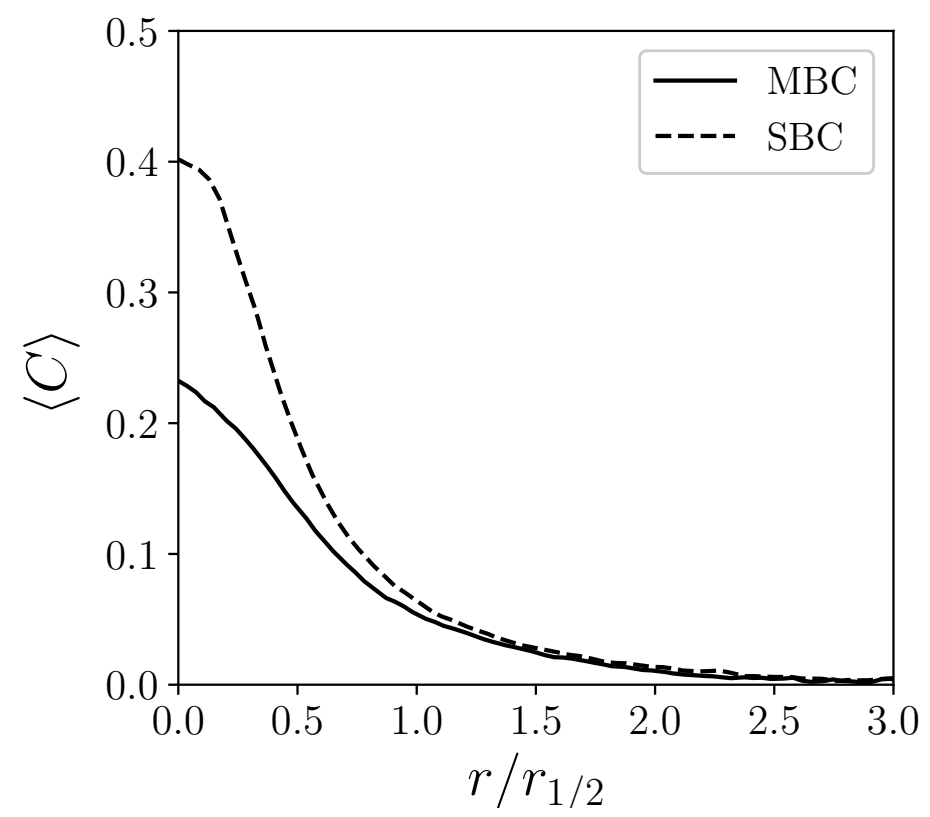

(b) Radial profile for the averaged Heaviside function.

Figure 7.2: Time averaged fields. 
the area $\Xi$ as :

$$
\Xi=\int_{0}^{R}\langle C\rangle d r
$$

which is indicative of the total mass that have been released into droplets (or air entrainment) and a useful mean of comparison. In this case, the integral ratio gives $\Xi_{S B C} / \Xi_{M B C}=1.28$ which, as it will be addressed in Section 7.4, can be related to the ratio in numbers of droplets generated.

The differences between the two cases are remarkably interesting, especially considering that similar conclusions where obtained in the previous study in Chapter 5, during a parametric study of the $L$ parameter in the SBC case. In Chapter 5, it is highlighted how larger turbulent structures are able to increase the perturbations on the liquid surface, inducing a earlier breakup and, overall, a earlier radial spread of the spray. Despite in the present study both cases have the same $L$ at the inlet, the comparison with the results in Chapter 5 suggests that the structure anisotropy of the MBC case generates effects similar to an increment of $L$. This consideration leads to the believes that the anisotropy of the turbulent structures of the MBC case can be associated with higher turbulent kinetic energy at the largest scales of motions.

The analysis made on Figures 7.1 and 7.2 led to the conclusion that at $x / D=25$ the flow is still not self-similar. It is although important to empathize that the concept of self-similarity in sprays, especially experimentally speaking and in the injectors analysis, is usually more relaxed than what discussed in the literature of jets [3]. In fact, for characterizations of injector's sprays, the Gaussian profile on the axial velocity is often considered a sufficient condition for self-similarity. On the other hand, this section clearly highlights how even for axial concentration of $\langle C\rangle>0.2$ such a condition is not reached. This needs to be further addressed in future studies with larger domains, able to capture the flow transition to the developed region.

\subsection{Determination of the Kolmogorov scale}

In this section, a specific focus on the turbulent scalar fields is used to improve the understanding of the behaviors previously identified by analyzing the mean flow statistics. In particular, the main focus of this analysis is the determination of the Kolmogorov scale, using the methodology described in Chapter 5. The importance of the Kolmogorov scale for the present study 
lies mainly in two reason. In a first place, it helps to asses the quality of the simulation, as it gives an idea on the amount of scales that can be resolved by the simulation. In fact, $\eta$ cannot be faithfully determined a-priori and its direct calculation after the simulation has reached convergence and stationary statistics is required.

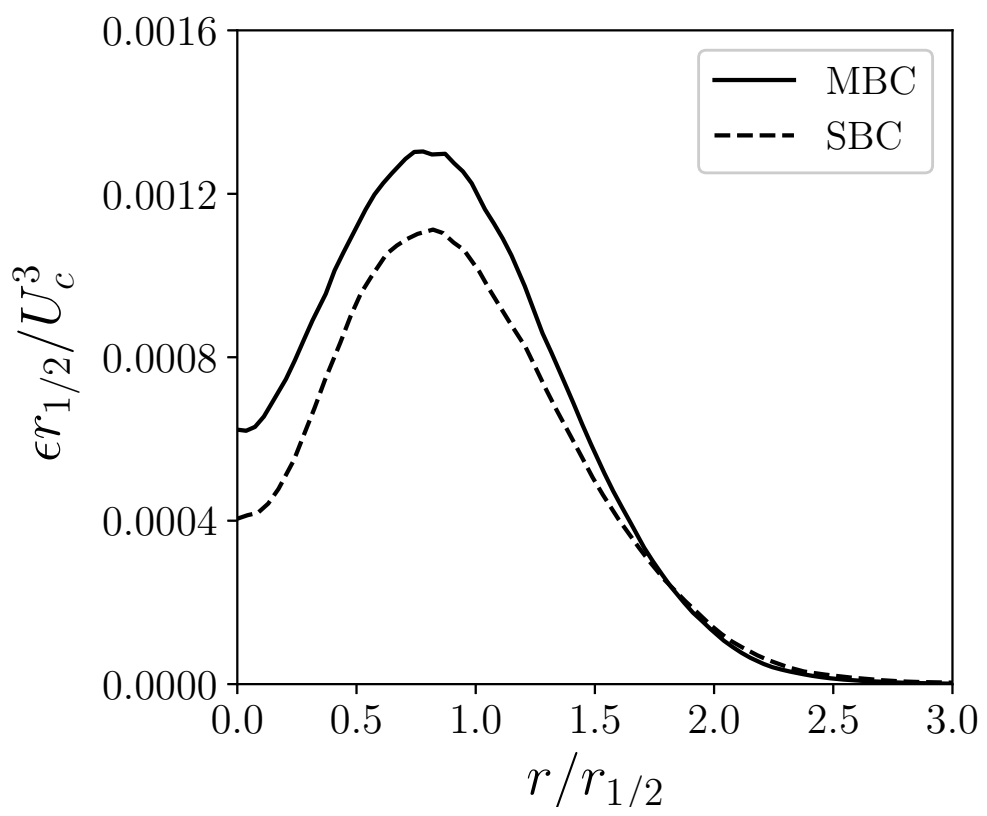

Figure 7.3: Energy dissipation rate.

A canonical approach is to study, for example, just the gas phase of the flow, here a more complete analysis is performed aiming to understand where the under-resolved regions of the simulation are and how/if it is affected by the primary atomization. In a second instance, $\eta$ provides a universal scaling factor which will allow to study several aspects of the simulation. For example rescaling the spectrum of the two simulations in order to improve the comparability. Ultimately, it also allows to ensure that the difference in the type of structures forced into the system by the BCs are not affecting in any way the small scales motion.

The energy dissipation rate is showed in Figure 7.3. Consistently with what has been showed previously, the numerical value of $\epsilon$ has been made dimensionless by multiplying it by $r_{1 / 2} / U_{c}^{3}$. On the other hand, in this case this data manipulation, which indeed improve the comparison with literature 
data, may be misleading. In fact the value of $\epsilon$ for the SBC case is almost 1.18 times the one computed for the $\mathrm{MBC}$ case. The most relevant reason for that is the difference in the $\langle C\rangle$ profile in Figure 7.2(b), which is used to compute the average viscosity field. Aside from this consideration, the two profile are indeed showing a similar behavior and consistent with what have been presented previously.

Figure 7.4 shows the radial profiles for $R e_{\lambda}$ (Figure 7.4(a)) and $\eta$ (Figure 7.4(b)). Here, $\eta$ is presented with its numerical value in order to improve the quality of the comparison and to provide a numerical reference useful for comparing similar simulations in future studies. Once again, the inverse proportionality $R e_{\lambda} \propto 1 /\langle\nu\rangle$ pushes towards the generation of a stronger turbulence field although do not yield any differences in the region where the smallest scales $\eta$ can be detected. As it can be noted, a flat region for $r<1.5 r_{1 / 2}$ is found, where the value of $\eta$ fluctuates between $0.5 \div 0.75$. As it can coherently be observed in $R e_{\lambda}$ this region corresponds to the highest turbulent intensity.

The extreme similarity in the value of $\eta$ deserves to be addressed. As assessed in literature, $\eta$ is independent of the large scale energy and, in general, from their anisotropic or isotropic behavior [4]. On the other hand, the analysis performed until this point are revealing a significant change in the overall spray behavior. Above all, the main difference is in the atomization behavior, that is clearly demonstrate in Figure 7.2(b) (and that will further addressed later). Most likely, the overall dynamic of the phenomena tends to balance out the liquid breakup and the velocity fluctuations. Although it is difficult to prove with a sound mathematical analysis of the data available, further proves will be provided later on.

\subsection{Spectral analysis of turbulence}

The spectra of axial velocity fluctuations is used here as an invaluable tool to understand the differences between the two cases. As described in Chapter 4 , the determination of the one-dimensional velocity spectra goes through the determination of the autocorrelation function. As the analysis of $f_{11}(\xi)$ (where $\xi=\delta \theta / 2 \pi$ is the dimensional radial distance function) can be revealing on the presence of velocity patterns, it is shown in Figure 7.5. Here, the axial velocity fluctuation autocorrelation function is displayed for various radial positions, where the corresponding values of $r / r_{1 / 2}, \eta$ and $R e_{\lambda}$ are showed in legend for each case. The radial position, of significant importance in sprays, is here expressed metrically to highlight an interesting behavior. Despite the difference in the one-point statistics showed in Section 7.1 a general collapse 


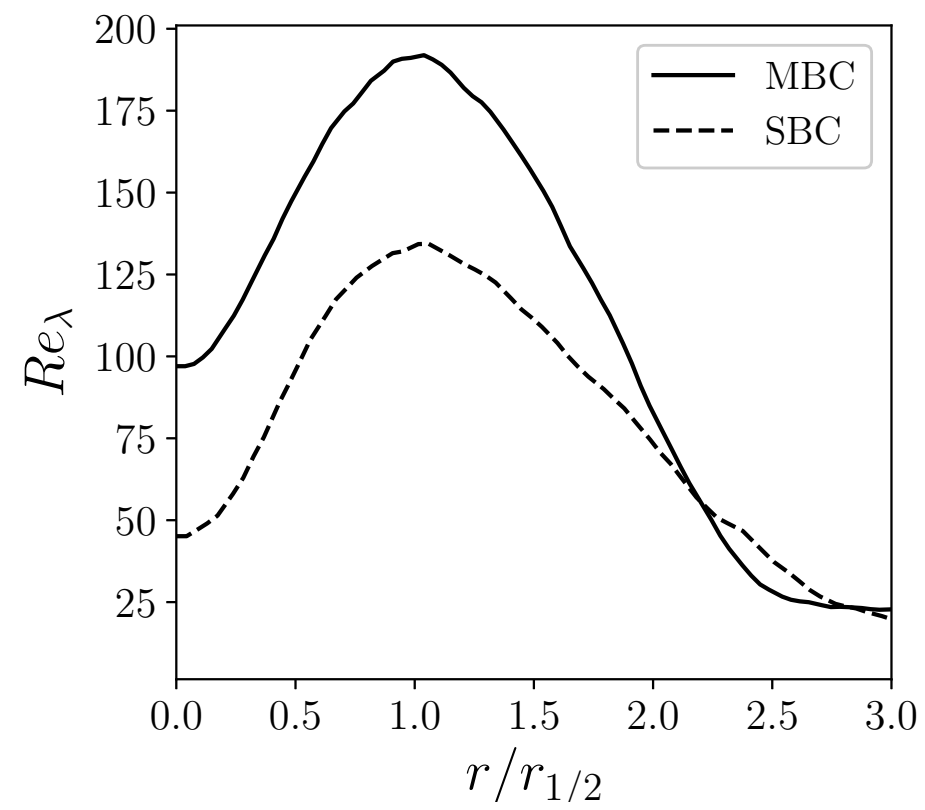

(a) Radial profile of $R e_{\lambda}$.

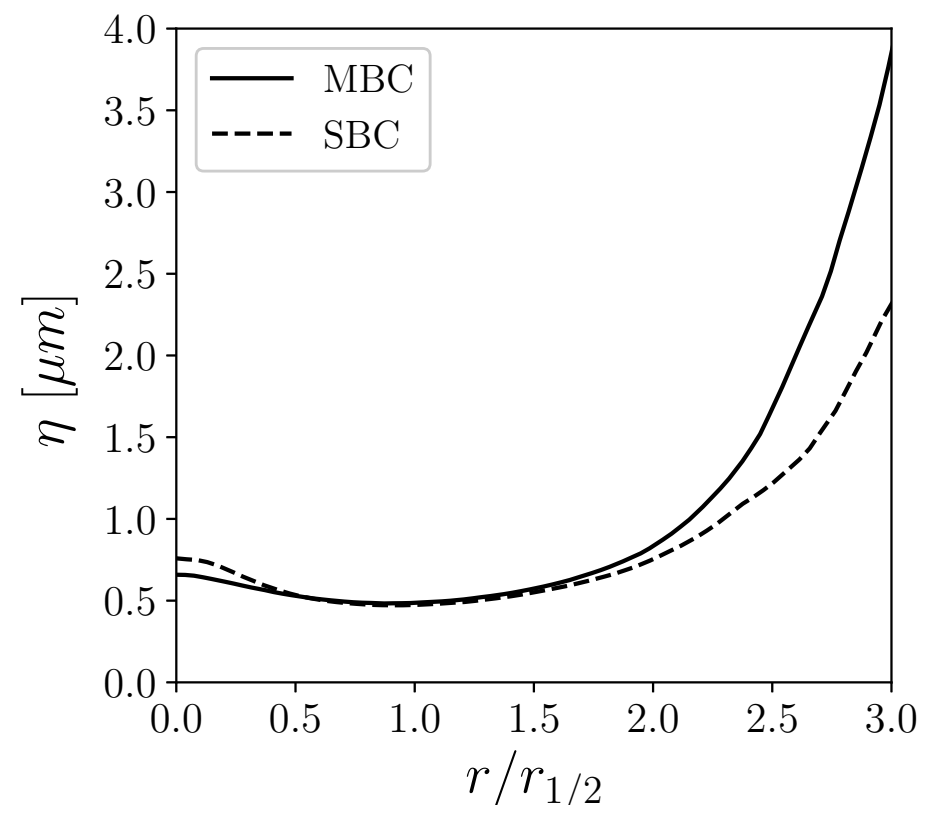

(b) Radial profile of the Kolmogorov scale.

Figure 7.4: Time averaged turbulent fields. 
is achieved by using $r / r_{1 / 2}$ as a dimensionless radial position. This is in common agreement with findings on sprays, jets and other free-shear flows. Due to a significant difference in the value of $r_{1 / 2}$ for the two cases, quite different behaviors are expected at the same radial absolute position. Figure 7.5 shows that this assumption is likely wrong or incomplete for higher two-point statistics. In fact, similar pattern can be observed. While it is clear that different energy content are displayed, it seems that at least in the final results, the sum of the harmonic function composing the signal is providing similarity in the patterns.
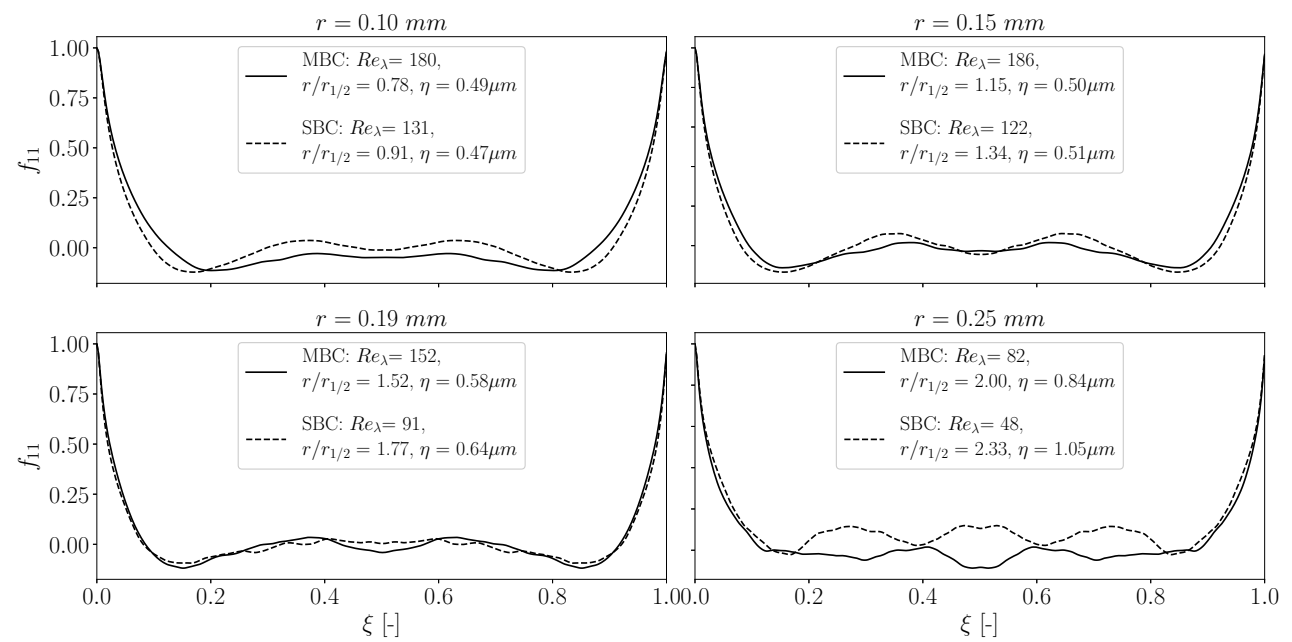

Figure 7.5: Axial fluctuating velocity autocorrelation. Each plot represents a radial position, expressed both in metrical values (in the title) and dimensionless value (in the legend). Legend also report the local value of $R e_{\lambda}$ and $\eta$ for reference with previous figures.

According to these considerations on $f_{11}$, and in order to improve the applicability and the readability of the results, the spectra of each absolute radial position is compared for the two cases in Figure 7.6. Each radial position is represented with a different color (continuous line for the MBC case and discontinuous for SBC ) and its local properties are reported in the legend. It is immediately clear that where the turbulence is fully developed $\left(R e_{\lambda}>100\right)$ the collapse of the each sets of curves is remarkably accurate. It can clearly be assumed that the flow is satisfying the hypothesis of local isotropy at smallest scales, hence losing the anisotropic behavior in case MBC. For all the fully turbulent sets of curves, the inertial range have a quite neat agreement with 
the $-5 / 3$ law, demonstrating that the energy transmission along scales is accurately modeled.

The dissipative range, on the other hand, is clean and no deviations are observable. In cases with solid particles analyzed as Lagrangian particles $\left(d_{v}>\eta\right)$ or point particle $\left(d_{v}<\eta\right)$ a deviation at higher frequencies is observed due to the absorption of part of the kinetic energy by the particle motions $[5,6]$. Spectra deviations were also experimentally found for bubbly flows [7]. In particle laden flows the spectra is computed on the fluid phase, which is likely to experience energy losses or increments during the transit of a droplet at a given location. Furthermore, bubbly flows spectra, which is usually a time-spectra, can be achieved by applying a Fourier transform to the velocity fluctuation time signal. This is mostly the main difference. When one performs the operation proposed in Equation (4.17) and average it over time a double "filtering" procedure is applied. In fact, Equation (4.17) already accounts for all the velocity products, whether liquid or gas is present at a certain location. Therefore only certain frequency patterns (of a frequency equal to the inverse of the droplet characteristic length) will be present in the autocorrelation. Finally, the time average of the autocorrelation provides a further dumping of the phase influence. This procedure is what really distinguished a pseudo-fluid approach by, for example, a hot-wire procedure. This approach allows to remove the disturbances introduced by the primary atomization, hence only evaluate the effects of the averaged turbulent structures. Finally, consistently with the cylindrical coordinate prospective described in Chapter 4 , the more the radial distance increase the more the cut-off frequency decrease.

Perhaps the most important differences in the spectrum lie in the low frequency energy range (large wavenumber, hence large structures). Here, it can be clearly observed that the $\mathrm{MBC}$ and the SBC cases present a clear tendency. In fact, the energy content of the MBC case is constantly higher than the SBC. This is a major difference between the two cases and the fact that the spectrum presents a perfect agreement at higher frequencies and a significant difference in the energy containing range is mostly the main explanation for what we have consistently observed until this point. In fact, as addressed in literature [8-10] the surface instabilities are closely connected with the nozzle turbulence, which generates radial deformations of the liquid phase that ultimately increase the shear forces and promote the breakup. The shear stress generates a number of hairpin turbulent structures that promote the formation and detachment of liquid structures [11]. Furthermore, the larger turbulent structures are responsible for the differences in such a process 
as they are the ones with a sufficient energy level to disrupt or perturb the liquid surface.

The larger structures contains more energy in the MBC case than the SBC case, hence the structures anisotropy favors the liquid breakup and the generation of more energetic eddies. On the other hand, for the same radial position, the neat collapse of the two cases spectrum highlights how, once the liquid structures start atomizing, the turbulence behavior for the two cases is almost identical. In other words, the larger structures relate closely to the detachment of liquid structures from the liquid core, while the smallest relate to the events that follow (liquid structures advection, secondary breakup, liquid structures coalescence, etc...). This consideration on different energy content at lower spatial frequencies explains why the liquid concentration is higher in the SBC case than in the MBC case Figure 7.2(b). Also, the similarity in the spectrum higher frequencies justify the collapse of the Kolmogorov scale in 7.4(b). This kind of behavior would have been difficult to spot without using a pseudo-fluid approach.

\subsection{Droplet size and properties distribution}

A first understanding of the simulation results can be provided by analyzing how the droplets are distributed. As already pointed out in [12], a droplet description can be reduced to its position $\mathbf{x}_{\mathbf{d}}$, its velocity $\mathbf{u}_{\mathbf{d}}$ and its diameter $d_{v}$. This determines, in the most applicable scenario, a 7-dimensional space for the droplet description. In literature of point-processes systems, such a representation is classified as a Klimontovich description of the system. While for many fluids some of this dimension can easily be lumped (e.g. $\mathbf{x}_{\mathbf{d}}$ in particle laden isotropic turbulence boxes [13]) sprays displays, at least, 2 main direction of development, the axial and the radial. For this reason, a first idea of the atomization patterns can be provided by understanding which is the typical droplet size distribution and how many of the kinetic energy of the liquid core is retained by atomized droplets.

Figure 7.7 shows the probability of droplet size distribution expressed as $\mathcal{P}_{d_{v}}=N / N_{\text {tot }}$ where $N_{t o t}$ is the total number of droplet. In a first place, it must be noted that the MBC case displays more atomization than the SBC case, given that $N_{t o t, M B C} / N_{t o t, S B C}=1.2$. This should not be discredited as marginal, as such an increment in atomization means a significantly higher mass loss in the spray core, hence a significantly higher loss in the axial momentum on the centerline. In this case, it is evident that the turbulent homogeneous structures induced by the SBC case are less prone to trigger at- 


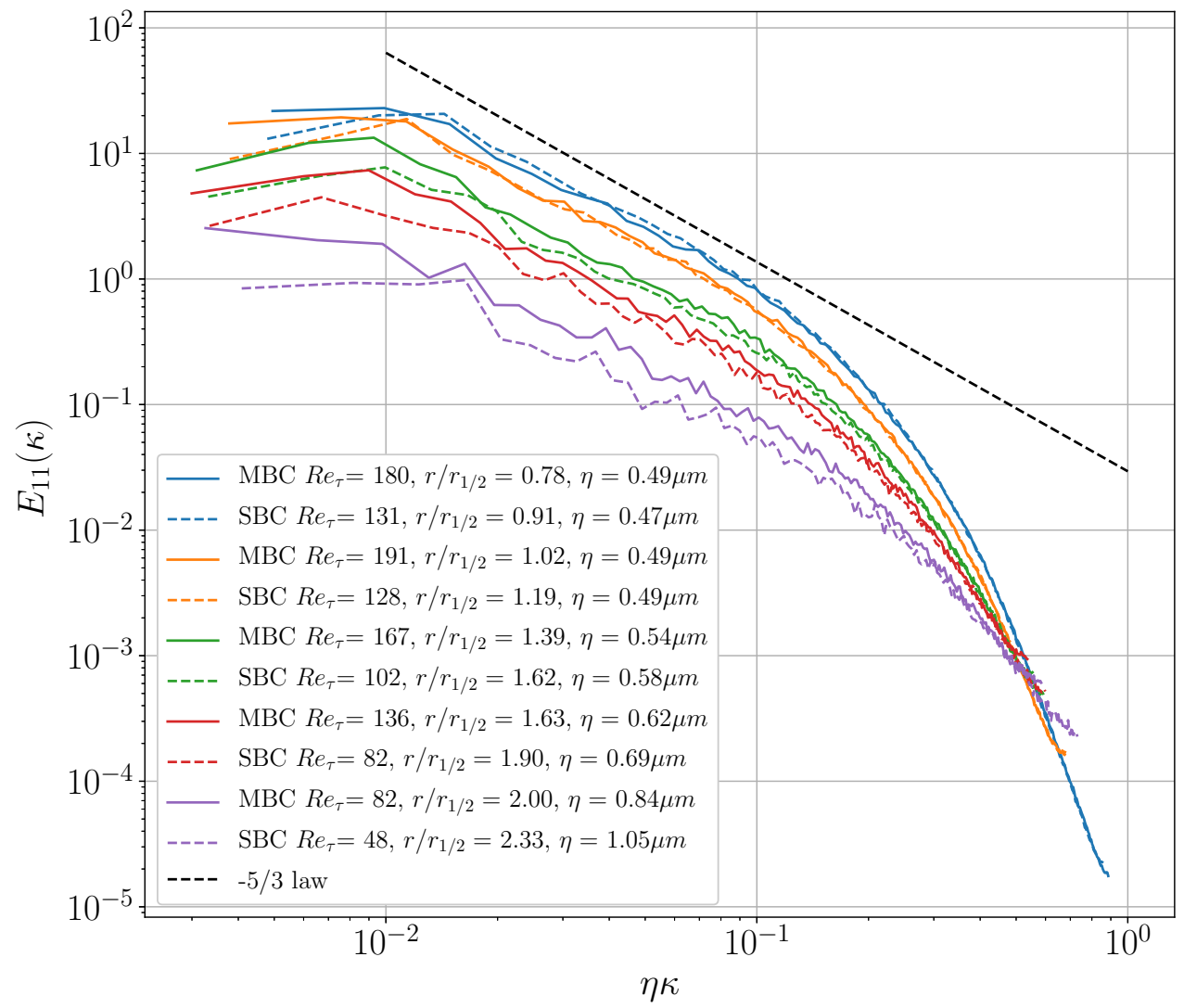

Figure 7.6: Axial fluctuating velocity autocorrelation. Each plot represents a radial position, expressed both in metrical values (in the title) and dimensionless value (in the legend). Legend also report the local value of $R e_{\lambda}$ and $\eta$ for reference with previous figures. 


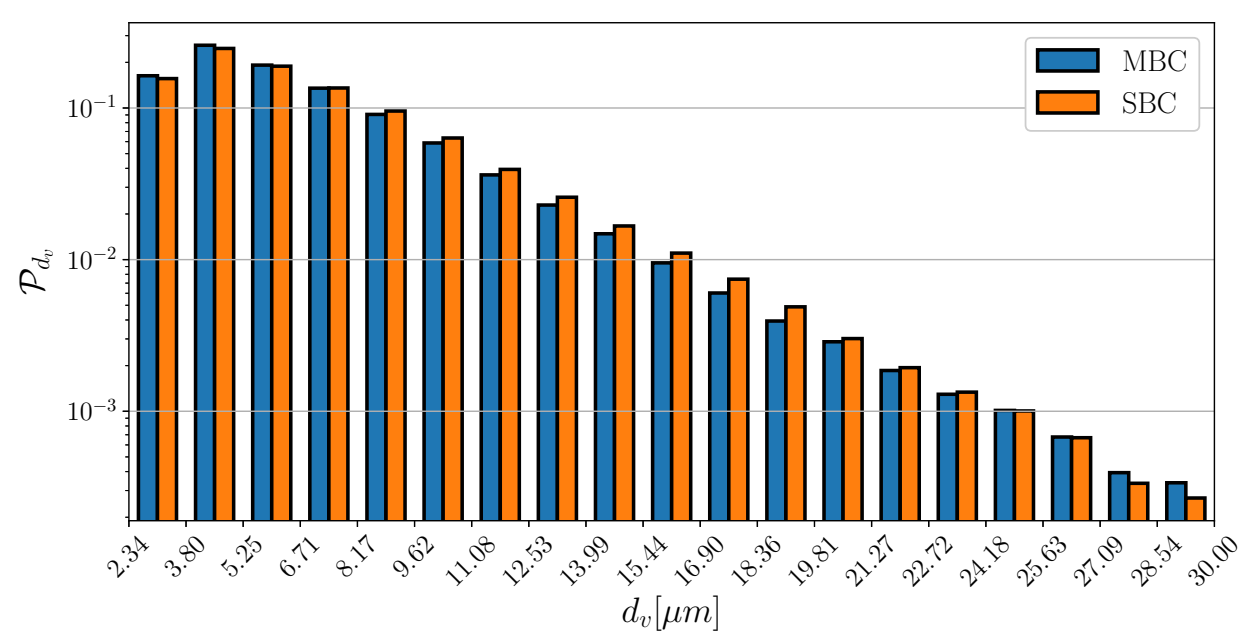

Figure 7.7: Probability of droplet droplet size distribution.

omization than the anisotropic structures produced in MBC. The meaningful ratios used to study this simulations are summarized in Table 7.1

$$
\begin{array}{ccccc} 
& r_{1 / 2} & U_{c} & \Xi & N_{d} \\
\frac{M B C}{S B C} & 1.16 & 0.95 & 1.28 & 1.2
\end{array}
$$

Table 7.1: Simulation lumped quantities ratio summary.

Aside from the total droplet count, Figure 7.7 highlights a interesting aspect of the atomization phenomena. In fact, the probability function $\mathcal{P}_{d_{v}}$ is remarkably similar for the two cases suggesting that, despite total number of droplets differs significantly, the way in which the ligaments forms and the spray breakup occurs follows a similar pattern. Moreover, the number ratio between the total number of droplet is consistent with the ratio between the concentration integral $\Xi$. This similarity helps to assess the quality of the droplets analysis (as all the mass lost has to convert into generated droplets). As already addressed in Section 7.3 the only significant differences in the onedimension kinetic energy spectrum can be located at the largest scales. In this context, this could lead to the conclusion that the larger structures are responsible for the amount of liquid that detaches from the liquid core. Is then likely that, once the larger liquid structures are detached from the liquid spray core, smaller eddies acts as surface perturbations that prompt atomization and further breakup. This hypothesis, although difficult to definitively 
prove with this simulations datasets, would be in agreement with the Kolmogorov hypothesis of log-normal distributions resulting from fragmentations and its consequent fractal behavior, observed in liquid sprays by $[14,15]$ and elegantly summarized in [16]. On the other hand, its proof would rely on droplet tracking and breakup detection which could be very computational demanding, especially in conjunction with the extraction of the turbulence statistics reported above.

As a droplet detaches from the liquid core, two possible scenarios are possible from a kinematic standpoint. Either the droplet is advected by the main flow, or is deviated towards the spray periphery. Being this study carried over in the dense region, where high average density can still be found on the spray axis, the fist scenario is quite likely to occur for droplets that have just detached from the liquid core and that have not yet reached a significant radial penetration. Furthermore, as the radial velocity component is still significantly less than the axial are (less than, for example, reported in [2]) and considering a rapid decaying Gaussian axial velocity profile, a droplet will rapidly lose velocity the further it gets from the spray axis. These considerations suggest that the droplet kinetic energy $E_{k}$ is a good indicator to have an understanding of the behavior of droplets in the 3 dimensions, $\mathbf{u}_{d}$, by lumping them via a physical parameter.

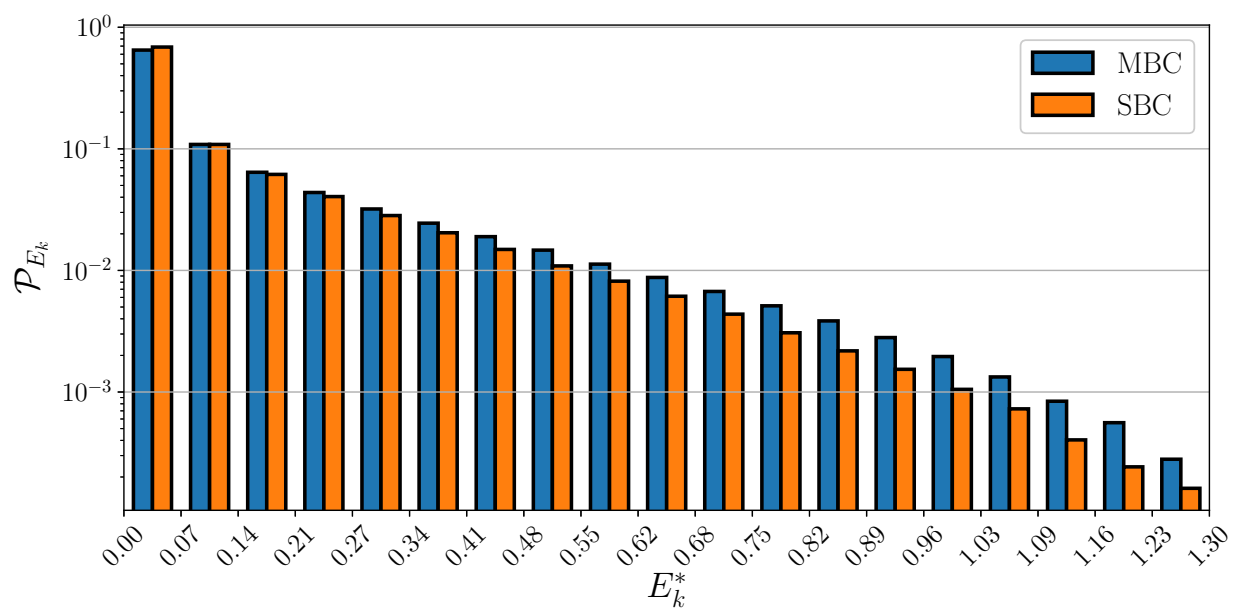

Figure 7.8: Probability of droplet kinetic energy distribution. The kinetic energy is made dimensionless by dividing by the injection kinetic energy $E_{i n j}=1 / 2\langle U\rangle^{2}$. 
Figure 7.8 shows the probability function for the droplet kinetic energy. In this figure, the kinetic energy of each droplet is made dimensionless by dividing it for the injection kinetic energy $E_{i n j}=1 / 2\langle U\rangle^{2}$, giving $E_{k}^{*}=E_{k} / E_{i n j}$. The expectancy of $\mathcal{P}_{E_{k}}$ at low kinetic energy values is significantly higher than other values, suggesting that many droplets have reached a quasi-rest state, hence a significant distance from the spray axis. Discussions available in literature $[8,17]$ suggest that these particles are generated by the curvature of the spray front into the typical mushroom-like shape. The peripheral liquid sheet is then teared by aerodynamic shear given by the large toroid-eddy generated on the front. This eddy usually displays $|\mathbf{U}| \approx 1.5\langle U\rangle$ tangential to the toroid and in the opposite direction of the main flow. Once this transient phenomena is passed, the velocity of the droplets generated by the spray front rapidly decay due to the aerodynamic drag provided by the spray core. These droplets are likely the ones that display a very small $E_{k}$ in Figure 7.8.

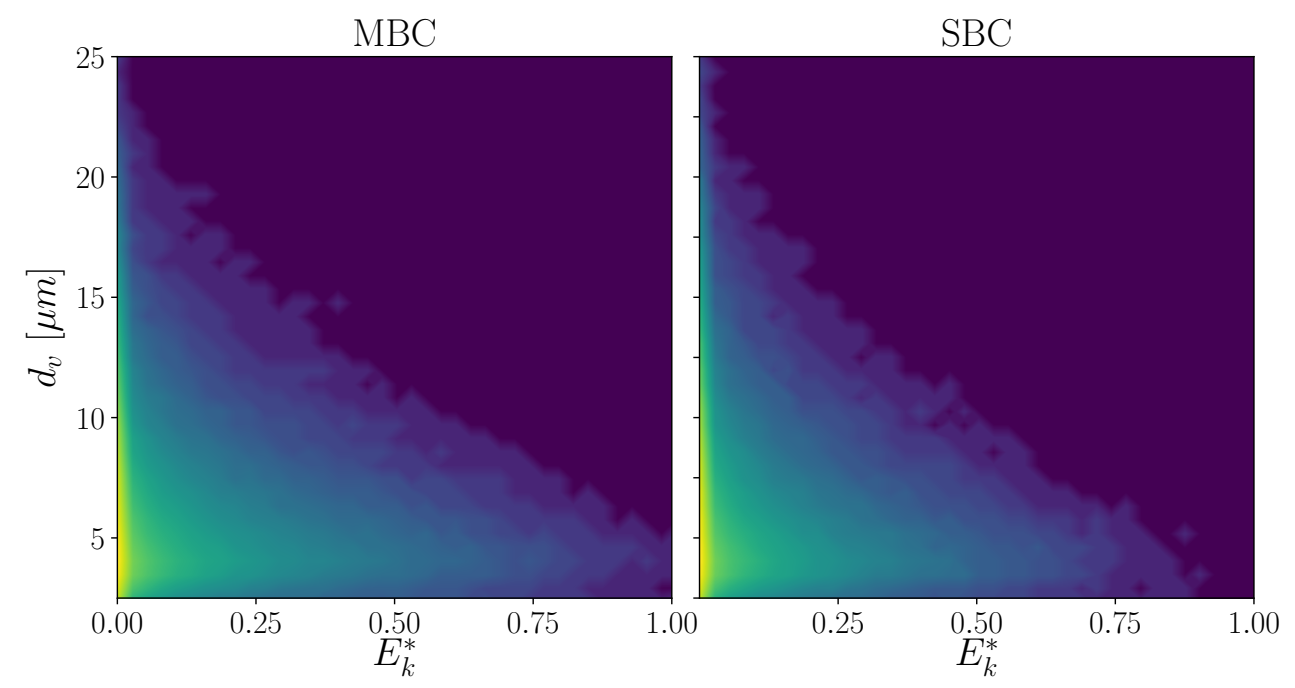

Figure 7.9: Joint PDF of droplet diameter $d_{v}$ and dimensionless kinetic energy $E_{k}^{*}$

To have a better insight on how the kinetic energy is distributed among droplets, Figure 7.9 shows the joint PDF for the dimensionless kinetic energy $E_{k}^{*}$ and the droplet diameter $d_{v}$. In order to improve the data readability, the contour coloring is logarithmic and the values are divided by the maximum value, hence no colorscale is reported. Figure 7.9 shows that, despite droplets of any diameter have the possibility of having very low kinetic energy, most of them are among the ones with the smaller diameter. Once again, the 
MBC case and the SBC case show a consistency in the described patterns. Finally, the resulting PDF from Figures 7.7 and 7.8 is showed in Figure 7.10 where a neat log-normal is shown for the PDF of the droplet diameter PDF in Figure 7.10(a) and an exponential decay for the kinetic energy in Figure 7.10.

The existence of expectancy of droplets with kinetic energy higher than the injection kinetic energy (in Figure 7.8) also needs to be discussed. Although this may seem physically unrealistic, its reason lies in Figure 3.3. In fact, the mean velocity profile imposed at the spray inlet generates a discrepancy between the the bulk and the centerline value of the Reynolds number, such as $R e_{c} / R e_{b}=1.35$. The velocity decay discussed in 7.1 shows also that such a droplet could be generated everywhere in the spray axial location due to its slow velocity axial decay.

Aside from the singularities in the extreme regions of the flow explained above, Figure 7.8 shows an exponential decay of the number of the kinetic energy distribution over droplets.

The final set of variable that need to be addressed in the Klimontovich approach are the droplets coordinates, which are going to be addressed in next sections.

\subsection{Droplet radial position}

The mere description of the radial position of each droplet (which examples can be found in Chapter 5) may be interesting for general spray characterization, but is insufficient for a statistical description of the spray. In fact, round sprays are conical in nature at sufficiently high Reynolds numbers and their spray angle changes with the considered axial position, as described in [18]. In particular, the spray cone angle changes significantly, depending on whether the dense region or the dilute region are considered. In the first case, it is difficult to define a spray cone angle as in experimental visualization. When the spray enters the dilute region it is easier to define a set of straight lines defining the spray cone projection over the image plane. For this reason, the droplet radial distribution is showed in conjunction with the corresponding axial position which gave a complete understanding of the space occupied in the pseudo-cone described by the spray.

Figure 7.11 shows the joint PDF for the radial position (divided by its axial position) and the axial position (divided over the total x-length $l_{x}$ ). As done before, the contour is logarithmic and made normalized by its larger value. This figure shows that for $0<x / l_{x}<0.37$ in both cases no angle is yet 


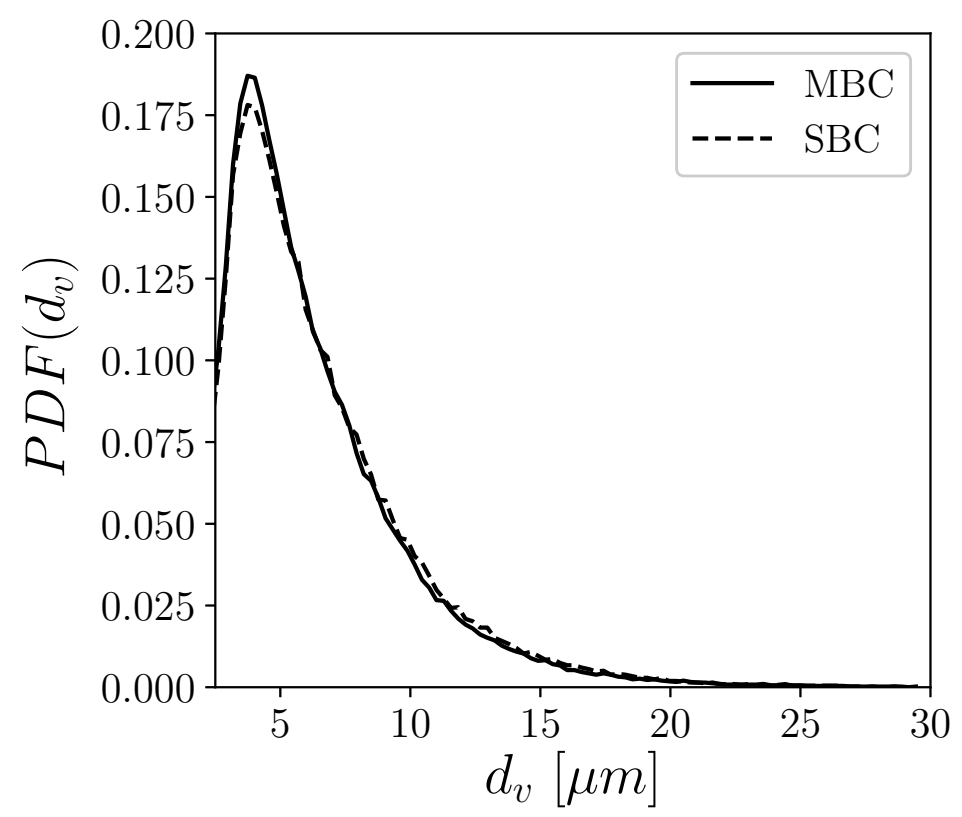

(a) PDF of droplets diameter.

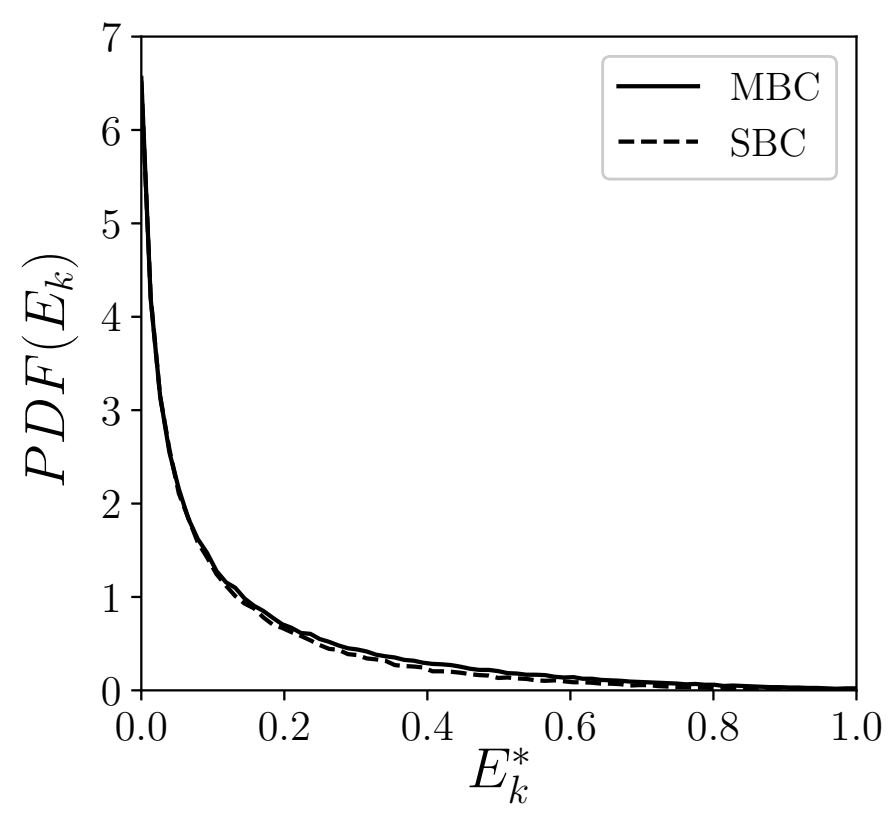

(b) PDF of dimensionless kinetic Energy.

Figure 7.10: Time averaged fields. 

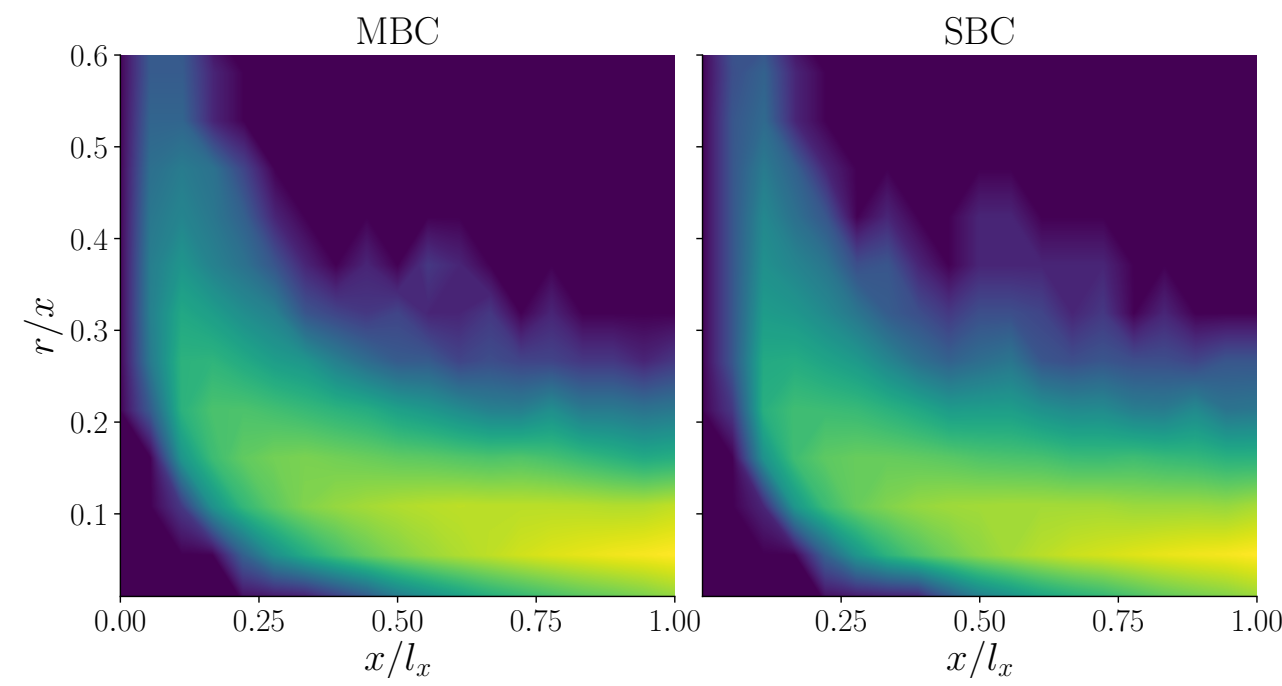

Figure 7.11: Joint PDF for the droplet radial position $r$ (normalized by its axial position $x$ ) and axial position $x$ (normalized by its axial total length $l_{x}$ ).

formed. this region shows a small number of droplets which are pushed quite far from the spray axis. The analysis of the time sequence of the snapshots reveals that these droplet are not generated in the spray when the statistical stationary state is reached. In fact, these droplets are generated by the spray tip during its transient penetrations and, for reasons explained previously their kinetic energy slows down quite fast until they reach a negligible velocity in comparison with the spray characteristic time. As a matter of fact, similar values for the tip radius during the transients are found in [17].

For $x / l_{x}>0.37$ there seems to be a convergence of the droplet dense region towards a well established pattern. In the radial region where $r / x<$ 0.25 no uniform droplet distribution is yes reached. that is because, as it is already discussed for the velocity profiles, the region is still dense and a self-similar spray behavior is not yet stably reached. It is expected that a more uniform droplet distribution can be reached in the dilute region, with a Gaussian distribution for the droplet radial position. Here, in both cases, no such a behavior has been reached while a dense region of droplet can be found for $0.37<x / l_{x}<1$ which, for all our intents, determines the atomized region in which most of the droplets are located.

In general, Figure 7.11 shows a distinct pattern for both cases and it reinforces, once again, the idea that no significant differences in the atomization behavior can be observed aside from the total amount of atomization. Once 
again, it is interesting to notice that the larger turbulence scales have no significant effect on the general droplet spread behavior aside from the total number of droplets generated.

\subsection{Spray axial development}

Figure 7.11 already gave a first grasp of how the droplet are distributed axially, but still the total number of droplets at each radial position should be addressed.

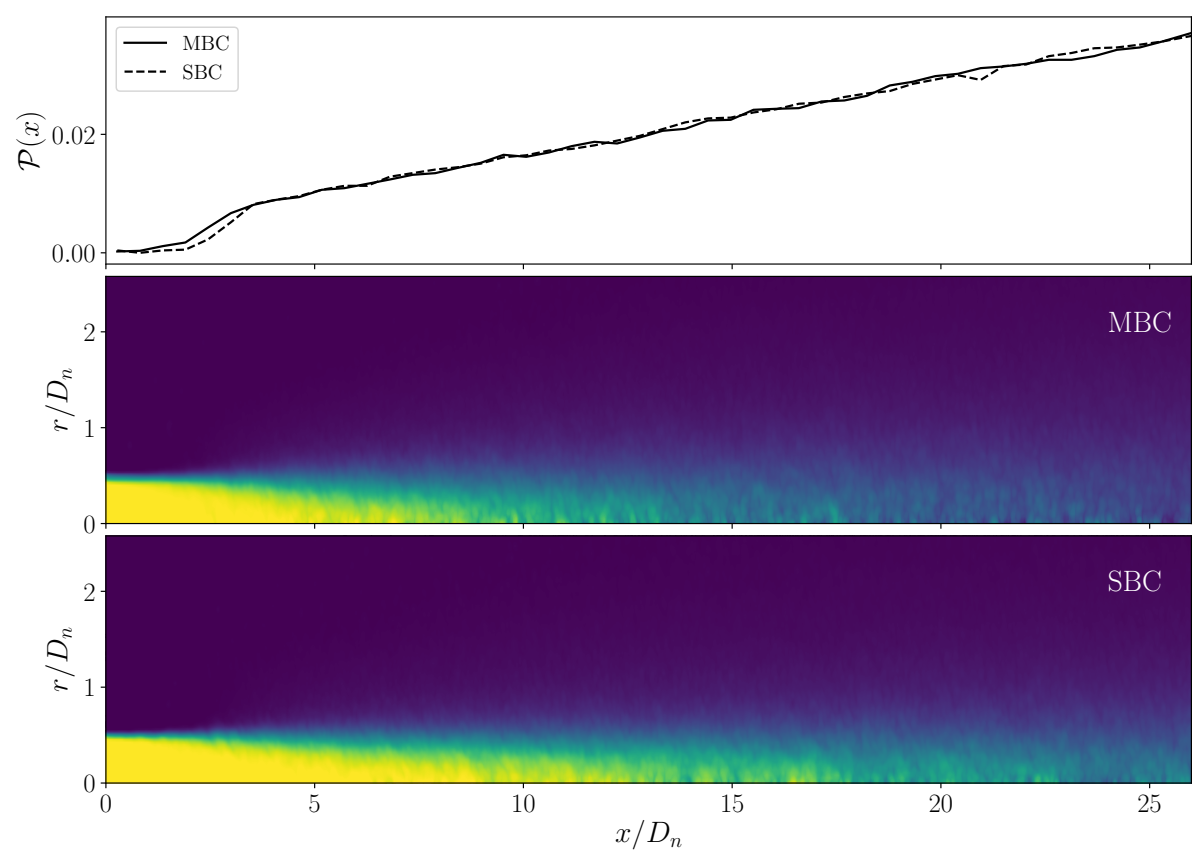

Figure 7.12: From top to bottom. (top) Probability $\mathcal{P}(x)$ of droplet presence as a function of axial position. (middle and bottom) Simulations snapshots of the volume fraction as a function of the radial position as well as the axial distance, both normalized by the nozzle diameter $D_{n}$. The $x$-axis of the figures are synchronized.

Figure 7.12 shows the probability $\mathcal{P}(x)$ of finding droplets at a given axial position (normalized by diameters $D_{n}$ ); below the curve, two time-averaged contour of the volume fraction in the simulation are showed. After a non 
atomized region ( for $x / D_{n}<2$ ) both cases start atomizing and, after a steep region at $2<x / D_{n}<5$, the probability $\mathcal{P}(x)$ for the two cases collapse again, into an extremely similar behavior. Despite this similarity, the axial timeaverage of the volume fraction decays significantly faster in the MBC case. This behavior is in agreement with what discussed until now on the similar atomization patterns. Another interesting consideration can be made over the steeper region of $\mathcal{P}(x)$. In fact, a certain correspondence can be found between the start of such a region and the axial shrinking of the liquid region, although the liquid region does not end when the steeper region stops. This is in contrast with what deduced in experimental analysis ( e.g. [18] ) and will need to be addressed in further studies.

The comparison of the volume fraction also support the idea previously stated, that the low frequency turbulence (larger scales) is responsible for the large liquid structures detachment while higher frequencies (smaller scales) generate the successive fracture of smaller liquid structures. Furthermore, it is interesting to understand how the droplet size is distributed at each axial distance. In fact, this would help to understand what are the drivers which generate atomization at each axial position.
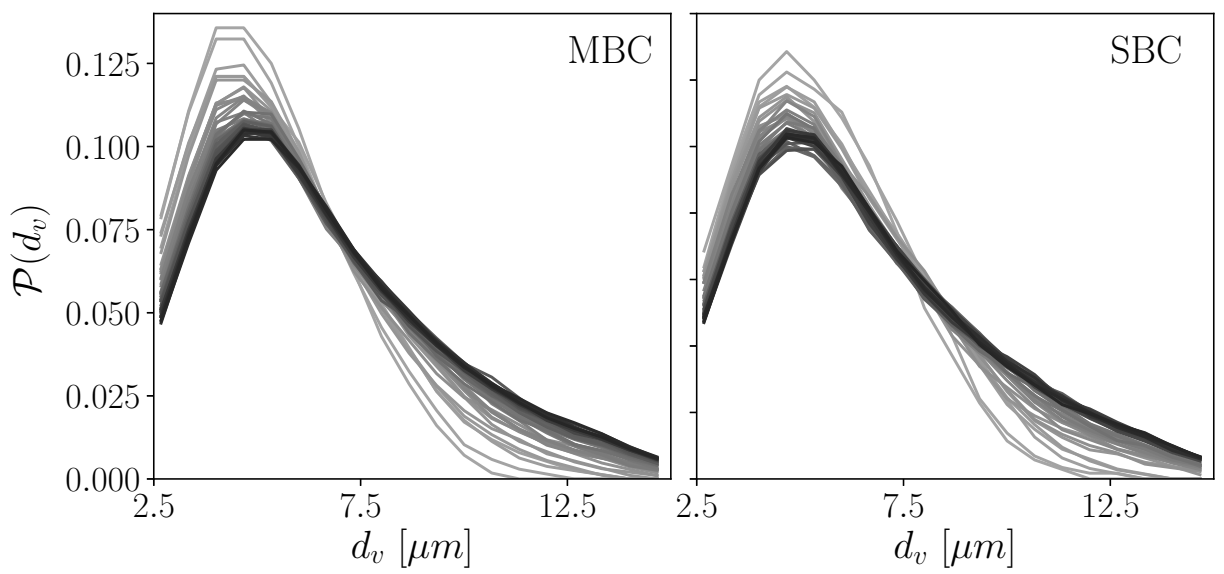

Figure 7.13: Probability $\mathcal{P}\left(d_{v}\right)$ for droplet size. The lines go from gray to black for $x \rightarrow l_{x}$. The axial distance goes from $x / D_{n}=2.5$ to $x / D_{n}=25$ according to the grayscale

Figure 7.13 shows the probability function $\mathcal{P}\left(d_{v}\right)$ at various axial position from $x / D_{n}=2.5$ to $x / D_{n}=25$ at increasing grey-scales. The number of bins for the droplet diameter has been reduced to increase the curve resolution as less droplets were available at each axial position (especially at lower values of 
$\left.X / D_{n}\right)$. As previously described, it is interesting to notice that at early axial positions most of the droplets generated are of small size where larger $d_{v}$ are almost non existent. Again this is because, as previously assessed, most of the droplets in this region are just created by the spray tip, which is a isolated phenomena in the simulation transient. While increasing the axial position, the curves converge over a more evenly distributed diameter size and converge towards a results similar to what has been obtained in Figure 7.7. Again both curves converge towards the same distributions although, for low penetrations, the MBC case is more biased towards the generation of smaller droplets.

\subsection{Generation of droplets over time}

Another revealing analysis that may be helpful in understanding how the dynamic of the atomization is affected by the different boundary conditions is the temporal analysis of the atomization. While many approaches could be used for this purpose, we chose to be consistent with the methodology used in Sections 7.4 and 7.6 and show, in a first place, the temporal generation of droplets grouped by size.
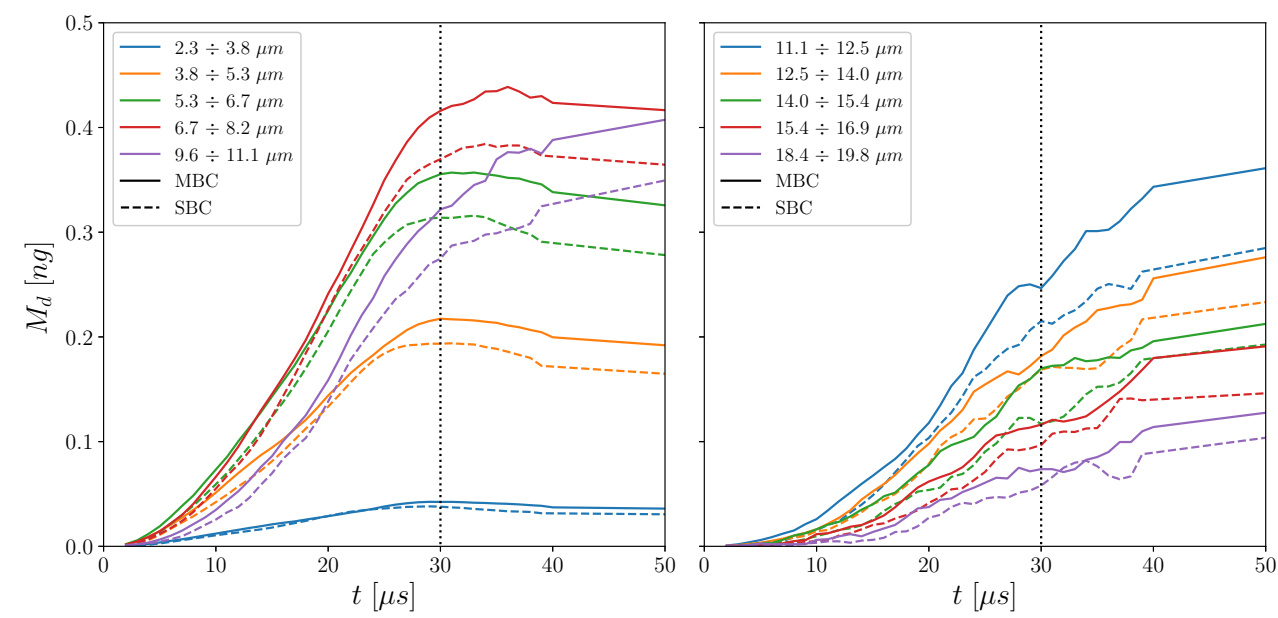

Figure 7.14: Total droplet mass divided by droplet size range, displayed as a function of time. The 2 plots represents the same study at different ranges and have been divided for clarity.

Figure 7.14 shows the generation of droplet within a size range (reported in the legend) in terms of total mass per diameter range, $M_{d}$. The sets of 
curves have been divided over two figures to improve readability and only ranges with significant mass are showed. The spray tip reaches the end of the domain at $t_{\text {end }} \approx 0.27 \mu \mathrm{s}$ and the statistics showed in Sections 7.4 and 7.6, as previously discussed in Chapter 4, are taken for $40 \mu s<t<300 \mu s$. Until $t_{\text {end }}$, all droplets sets display a linear increment of mass, which is extremely neat for those sets where a high droplet number is available for statistics (e.g. $\left.d_{v}<10 \mu \mathrm{m}\right)$. The correspondent time-averaged slope, expressed as $d M / d t$ (for $t<30 \mu \mathrm{s}$ ) is showed in Figure 7.15(a). As obvious for this part of the simulation, all the derivatives are positive and a clear trend can be observed.

It is immediately evident that droplet sets that are of high relevance in droplet diameter distributions statistics, such as $d_{v}<5.5 \mu \mathrm{m}$ are less relevant from a total mass standpoint. In fact, it can be observed how significant importance is gained by droplets for which $5.5 \mu \mathrm{m}<d<12.5 \mu \mathrm{m}$. As there are no evidences that a sufficient mesh size has ever been achieved for fully turbulent atomizing sprays, this trend suggests that non-fully-resolved DNS simulations (at least from an atomization standpoint) may still be useful tools for providing data for sub-grid models and for improving the understanding of the spray behavior.

After the total spray penetration has been reached, still a quite clear trend can be observed as the simulation reaches a statistical stationary state. In order to do so, similarly to what previously discussed for the transient, Figure 7.15(b) shows mass generation per unit time for the different droplet size. As widely discussed before, we hypothesized that the smallest droplets are generated by the spray tip, therefore it is likely to see that their generation rate decrease. Such a phenomena can be appreciated for $d_{v}<10 \mu m$ where globally the total number of small droplets decrease. In fact, it is likely that aside from a reduction in their production, overall a significant amount of coalescence is occurring, justifying a negative value of the derivative. On the other hand, there is still a slight production of droplets for $d_{v}>10 \mu \mathrm{m}$ but significantly smaller than what achieved during the initial transient.

It is clear that most part of the droplets generation is reached in the transient phase of the spray before a complete penetration occurs. To show this behavior, Figure 7.16 the ratio between the total mass of droplets, $M_{d}$, and the total injected mass, $M_{i n j}$, over time. Before $t_{\text {end }}$ the total amount of atomized mass grows faster than the total mass injected. Overall, the MBC accounts for more atomized mass, although the ratio is less prominent than what discussed previously for the SBC case, showing that most of the droplets generating the discrepancy are of small size. When $t>t_{\text {end }} M_{d}$ increase way slower than the total amount of mass injected $M_{i n j}$ (which grows linearly with 


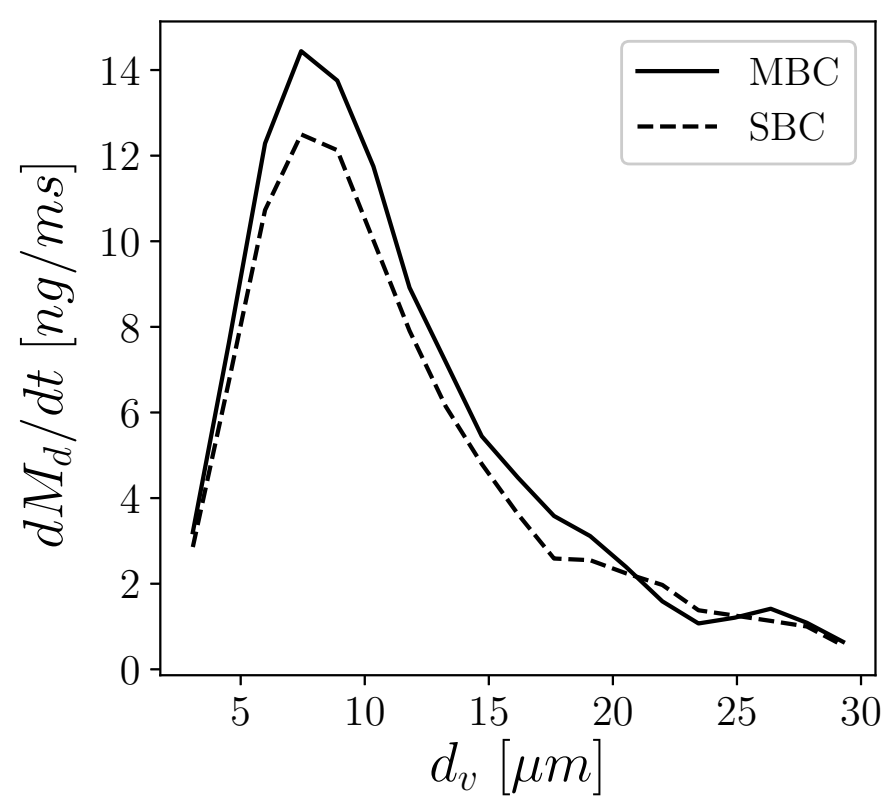

(a) Transient phase.

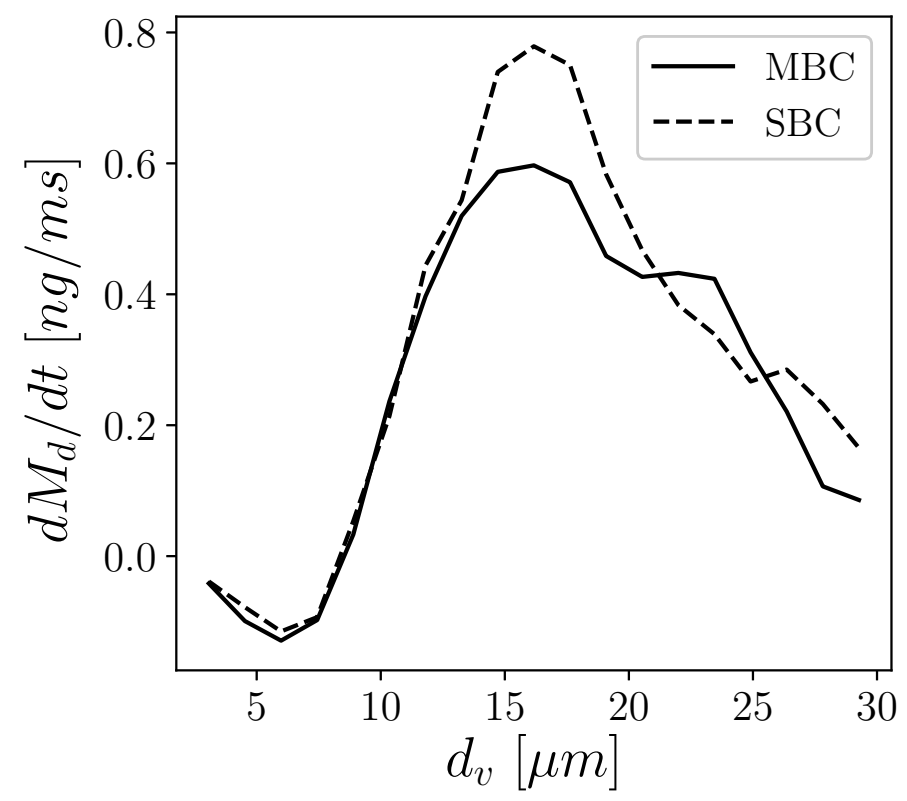

(b) Statistically stationary phase.

Figure 7.15: Averaged droplets generation rate as a function of the droplet diameter. 
time), hence the curves collapse toward an hyperbole.

Indeed this consideration highlights the fact that most of the droplets are generated by the transient phase. The aerodynamic shear, which is the main driver generating the turbulence necessary for triggering atomization is responsible just for a small amount of the droplet generated hence it is likely that the effects generated by the anisotropic structures of the MBC are far more important in the initial transient, although no easy explanation can be provided in lack of tools such as the spectral analysis, which require statistically stationary measurements.

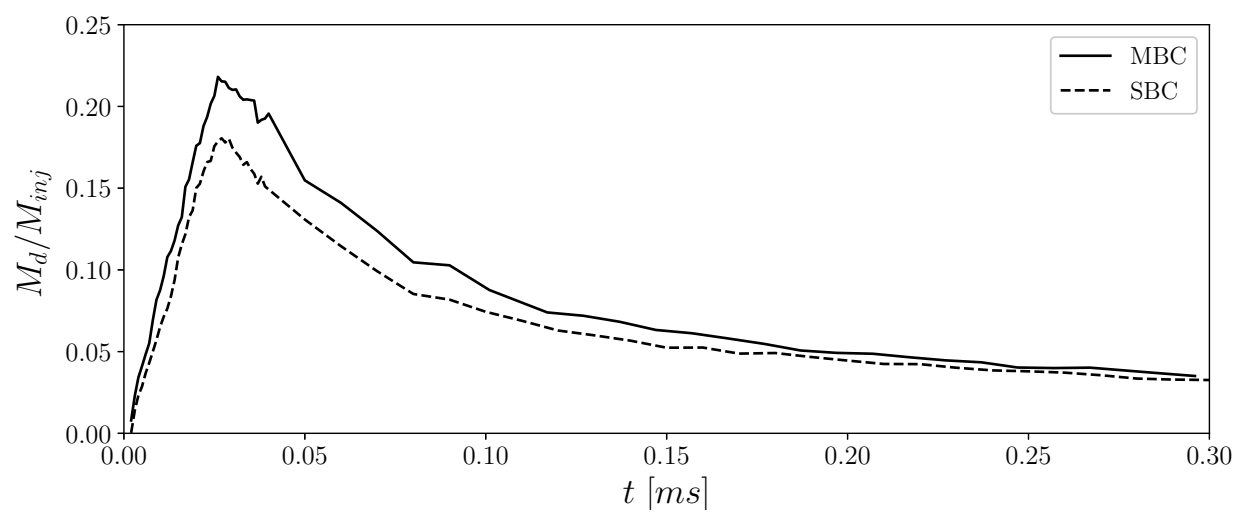

Figure 7.16: Total mass of atomized droplets over the total mass injected as a function of time.

\subsection{Radial Distribution Function analysis.}

One of the advantages of treating a spray as a point-process is that the droplets statistical location can be resumed by very few parameters in the disperse region. One of them, and maybe the most important, is the relative distance between droplets, namely the Radial Distribution Function (RDF).

As already mentioned in Section 7.4, the droplet discretization used here has the inherited possibility of treating the spray by means of a Klimontovich approach [12]. In this case, if the temporal evolution of the spray is neglected, the fine-grained statistical discrete function that describes the spray depends only on velocity $\mathbf{U}$, location $\mathbf{x}$ and diameter $d_{v}$. All three variables have been statistically characterized above, but no information has been provided on how droplets space among them. In statistical mechanics, as well as in point-process, this information can be summarized into the RDF. Intuitively, 

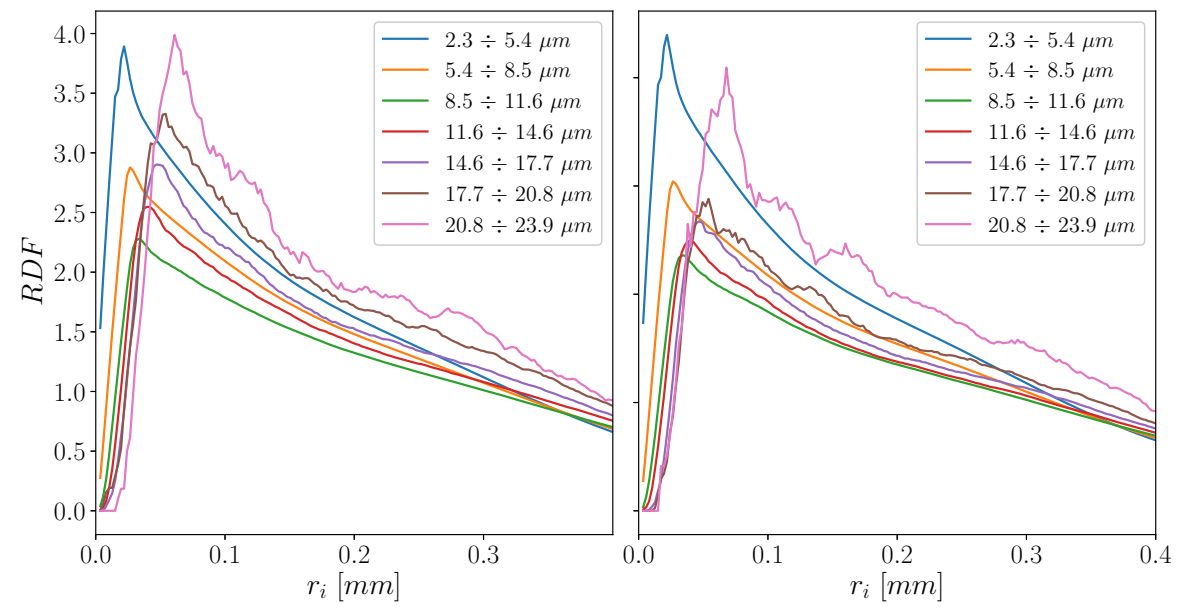

Figure 7.17: RDF for droplets ranges (reported in legend). On the left, the results of the $M B C$ case are showed while the results of the $S B C$ case are showed on the right.

this function characterizes the probability of finding a droplet at a specific distance from another one, given that all droplets positions are known and that each distance between a droplet pair is computed at their mass center. This probability can then be compared to the case in which all the given droplets are equally distributed along the domain. A simple mathematical description of this function can be given by:

$$
R D F\left(r_{i}\right)=\frac{N_{p, i}}{N_{p}} \frac{V}{\delta V_{i}}
$$

where $N_{p, i}$ is the total number of droplets pair at a distance contained in a sphere shell of radius $r_{i}$ and volume $V_{i}$ divided by the uniform droplet pair distribution $N_{p} / V$ where $N_{p}$ is the total number of droplet pair and $V$ is the total domain volume.

It is worth notice that the definition in Equation (7.2) is useful in experiments and in particle laden simulations, where the droplets uniform distribution has an actual sense, given the geometry of the physical problem. It is the author opinion that for sprays it should be revisited. The geometry of sprays, as already widely discussed, can be well characterized by a cone where the angle can be defined by the mass concentration radial distribution (see [18]). In a domain as the one used here, there will be a significant portion 
of the domain that will not be populated by droplets and that will offset the $\mathrm{RDF}$ function. On the other hand, the values obtained here are comparable with data found in literature $[13,19]$ but, in the author opinion, the real value should be higher. As the discussion of this topic could be complex in the dense region, this problem is destined to future works.

Figure 7.17 shows the RDF for the MBC (left) and the SBC (right) cases. As the number of smaller droplets $\left(d_{v}<15 \mu \mathrm{m}\right)$ is significantly high, smoother statistics are obtained, while for larger droplets a noisier signal is found. Nevertheless, it can be appreciated as very small droplets have the tendency to cluster together. As the droplet size increases their clustering decrease and a minimum is found for $d_{v} \approx 10 \mu \mathrm{m}$. Finally, as the diameter increase further, droplets again tend to form clusters. These trends are likely due to different phenomena. Smaller droplets are easier to advect due to their low mass, therefore tends to follow the small-particle behavior, hence forming clusters in low turbulence regions. Larger droplets are mostly formed in the spray core and have a significant inertia, as well as total mass. While these factors would lead to the conclusion that these droplets should disperse, the fact that most o the velocity in the spray core is still predominantly axial, these droplets are still penetrating axially, therefore their dispersion is relatively low.

\subsection{Conclusions}

In this section, the analysis of two different cases, namely SBC and $\mathrm{MBC}$, allowed to understand and quantify the influence of turbulent structures shapes, in principle described by the same integral quantities $L$ and $I$, on the spray breakup and atomization. The effects of this structure is quantified both from analyzing the consequent turbulent field generated as well as analyzing in depth the droplets properties statistics. The discussion performed in Sections 7.2 and 7.3 is particularly revealing. Here, data showed that an a-priori determination of $\eta$ for this kinds of multiphase flows is likely to be fairly inaccurate. This simple correlation in fact neglect the complexity of the flow in the mixing area of the dense region. On the other hand, the spectra demonstrates that the unresolved energy is a negligible percentage of the total one, hence the information loss do not prevent to obtain significant results. On the other hand the smallest scale for atomization on DNS is still to be reached and, as discussed in literature [20], will likely require the development of more suitable numerical techniques able to resolve up to the phase-interface thickness.

Despite this limitations in modern multiphase DNS simulations, some interesting contribution over the flow spectra have been achieved. In a first 
place, the pseudo-fluid method for obtaining spectra was used to achieve smooth spectrum and to understand how the energy distributes among scales at different radial positions of the spray. The same tool was used to quantify the differences in the Fourier coefficients representing larger structures, providing an explanation on the different breakup dynamics occurring in the two cases. The differences in the one-point statistics allowed to assess that the current domain is still far from reaching the full axial self-similarity described in literature for jets and sprays in the disperse region. Despite that, this section contributes in the understanding of the transition from near-field to far-field and how the velocity profiles behave when affected by different nozzles (here represented by the differences in the turbulent structure shapes).

The droplets analysis allowed to improve the understanding on how turbulence affects breakup. In fact, the small differences in probability (and probability density) functions highlights how $R e$ and $W e$, here constant for both the MBC and the SBC cases, are still the main parameters that need to be investigated in this flows in order to capture differences in the atomization behavior. While anisotropic structures trigger a significantly stronger atomization, the overall probabilities are not significantly different, showing that nozzle turbulence could be used to accelerate the spray breakup but not to control the atomization behavior if the same turbulence parameters are maintained. In fact, this analysis shows that although it is possible to force more breakup events by enhancing the nozzle turbulence and its behavior, the overall diameter distribution keeps been statistically similar, hence dictated by other physical phenomena.

Finally, the description of droplets size, generation, position (absolute and relative) and energy is widely discussed, opening the ground for statistical modeling and description of sprays.

\section{References}

[1] Álamo, Juan C. del and Jiménez, Javier. "Spectra of the very large anisotropic scales in turbulent channels". In: Physics of Fluids 15.6 (2003), pp. 41-44. DOI: 10.1063/1.1570830.

[2] Hussein J. Hussein, Steven P. Capps, and William K. George. "Velocity measurements in a high-Reynolds-number, momentum-conserving, axisymmetric, turbulent jet". In: Journal of Fluid Mechanics 001.258 (1994), pp. 31-75.

[3] Corrsin. Spectra and Diffusion in a Round Turbulent Jet. Tech. rep. 8. 1943, pp. 399-405. 
[4] Pope, Stephen B. Turbulent Flows. 2001. DOI: 10.1088/0957-0233/ 12/11/705.

[5] Sundaram, Shivshankar and Collins, Lance R. "A numerical study of the modulation of isotropic turbulence by suspended particles". In: Journal of Fluid Mechanics 379 (1999), pp. 105-143. DOI: 10.1017/ S0022112098003073.

[6] Burton, Tristan M. and Eaton, John K. "Fully resolved simulations of particle-turbulence interaction". In: Journal of Fluid Mechanics 545.2005 (2005), pp. 67-111. DOI: 10.1017/S0022112005006889.

[7] Prakash, Vivek N. et al. "Energy spectra in turbulent bubbly flows". In: Journal of Fluid Mechanics 791 (2016), pp. 174-190. DOI: 10.1017/ jfm.2016.49.

[8] Salvador, F.J., Ruiz, S., Crialesi-Esposito, Marco, and Blanquer, Ignacio. "Analysis on the Effects of Turbulent Inflow Conditions on Spray Primary Atomization in the Near-Field by Direct Numerical Simulation". In: International Journal of Multiphase Flow 102 (2018), pp. 4963. DOI: 10.1016/j.ijmultiphaseflow.2018.01.019.

[9] Jarrahbashi, D. and Sirignano, W. A. "Invited Article: Vorticity dynamics for transient high-pressure liquid injection". In: Physics of Fluids 26.10 (2014). DOI: 10.1063/1.4895781.

[10] Zandian, A., Sirignano, W. A., and Hussain, F. "Understanding liquidjet atomization cascades via vortex dynamics". In: Journal of Fluid Mechanics 843 (2018), pp. 293-354. DOI: 10.1017/jfm.2018.113.

[11] Jarrahbashi, D., Sirignano, W. A., Popov, P. P., and Hussain, F. "Early spray development at high gas density: hole, ligament and bridge formations". In: Journal of Fluid Mechanics 792 (2016), pp. 186-231. DOI: 10.1017/jfm.2016.71.

[12] Subramaniam, S. "Statistical representation of a spray as a point process". In: Physics of Fluids 12.10 (2000), pp. 2413-2431. DOI: 10.1063/ 1.1288266 .

[13] Park, George Ilhwan, Bassenne, Maxime, Urzay, Javier, and Moin, Parviz. "A simple dynamic subgrid-scale model for les of particle-laden turbulence". In: Physical Review Fluids 2.4 (2017), pp. 1-20. DOI: 10. 1103/PhysRevFluids . 2.044301.

[14] Marmottant, Philippe. "Atomisation d'un jet liquide par un courant gazeux". PhD thesis. 2001. 
[15] "The fractal geometry of round turbulent cryogenic nitrogen jets at subcritical and supercritical pressures". In: Atomization and Sprays 14.1 (2004), pp. 81-91. DOI: 10.1615/AtomizSpr.v14.i1.50.

[16] Gorokhovski, Mikhael and Herrmann, Marcus. "Modeling Primary Atomization". In: Annual Review of Fluid Mechanics 40.1 (2008), pp. 343366. DOI: 10.1146/annurev.fluid.40.111406.102200.

[17] Shinjo, J. and Umemura, A. "Detailed simulation of primary atomization mechanisms in Diesel jet sprays (isolated identification of liquid jet tip effects)". In: Proceedings of the Combustion Institute 33.2 (2011), pp. 2089-2097. DOI: 10.1016/j.proci.2010.07.006.

[18] Benajes, Jesus, Salvador, Francisco Javier, Carreres, Marcos, and Jaramillo, David. "On the relation between the external structure and the internal characteristics in the near-nozzle field of diesel sprays". In: Proceedings of the Institution of Mechanical Engineers, Part D: Journal of Automobile Engineering 231.3 (2017), pp. 360-371. DOI: 10.1177/0954407016639464.

[19] Sahu, S., Hardalupas, Y., and Taylor, A. M.K.P. "Droplet-turbulence interaction in a confined polydispersed spray: Effect of turbulence on droplet dispersion". In: Journal of Fluid Mechanics 794 (2016), pp. 267-309. DOI: $10.1017 / \mathrm{jfm} .2016 .169$.

[20] Pitsch, H. and Desjardins, Olivier. "Detailed Numerical Investigation of Turbulent Atomization of Liquid Jets". In: Atomization and Sprays 20.4 (2010), pp. 311-336. DOI: 10.1615/atomizspr.v20.i4. 40. 


\section{Chapter 8}

\section{Conclusion and future works}

In this chapter, the main scientific conclusions of this thesis will be highlighted, with a focus on how these lead to future works and further analysis. A synthetic resume of the findings of each chapter will be performed with a more open view on the overall works, hence for specific conclusions it is suggested to check the final section of each of the analysis chapter.

While, along the thesis, many insights on the physical problem have been provided, no extensive discussion over the computational aspects of this thesis have been performed. In fact, the whole discussions provided on the simulation results presented previously, accounts for approximately 3 millions core-hours of simulations and $60 \mathrm{~TB}$ of raw simulation data. The analysis of these dataset required in fact a substantial development of toolboxes for data handling, storage requirements reduction and parallel processing. Furthermore as discussed in Appendix A, a fast solver has been developed in order to account for future simulations. The development of all this tools, which will be discussed further in next sections, was made in order to create a useful framework for continuing the research within the research group in CMT-Motores Térmicos. On the other hand, its full discussion has been avoided as the main focus of the work is purely physical and it was among this work priority to keep the discussion as clean and linear as possible.

Finally, based on what discussed above, few suggestions on future works will be provided. Part of the activity that will be proposed are already ongoing within the research group and will be briefly discussed. Furthermore, the analysis performed in the previous chapters open the path for more funda- 
mental questions on sprays, primary atomization and multiphase turbulence, hence these fields will be addressed as well.

\subsection{Conclusion over physics analysis}

\subsubsection{Considerations over the inflow boundary conditions}

As a significant amount of the work has been centered over the discussion, selection, implementation and calibration of the inflow boundary condition, some considerations over the choice among the one discussed should be performed. As often in physics and engineering, no "perfect solution" is available and the answer on whether to pick the MBC or the SBC is really up to the goal of the study and the amount of experimental data available for comparison (both for the nozzle flow as well as for the atomizing spray). One of the most important driver in the decision, in fact, is whether or not data of the nozzle flow are available, allowing, for example, comparison with other theoretical studies (e.g. DNS or experiments of boundary layer and pipe flows). The limited informations over the nozzle flow is, in fact, often limiting in the validation phase, hence many different researchers chose different approaches.

As discussed in Chapter 7 a very interesting conclusion is that the PDF and, in general, the study of droplets do not changes significantly by changing the boundary condition type (mapped from LES or synthetically generated) and whether or not the anisotropy of the nozzle structures is accounted for the boundary condition. This means that the physical process that describes the atomization is not affected in its behavior by the boundary conditions. For fundamental works, where experimental PDF of droplets are available for comparison, the analysis of Chapter 7 highlights how whether the $\mathrm{MBC}$ or the SBC is used the same statistical behavior is achieved on both droplet size distribution, kinetic energy, spatial distribution and generation over time. This is an important result to keep in mind when designing a simulation that aims to compare the statistical behavior of atomization.

Unlike the "robustness" of the droplet statistics (and the physical process that cause them), the atomized mass changes significantly. Many reasons can be found for that. The different properties of the turbulent structures, whether homogeneous or anisotropic, may be a possible cause, as Section 7.3 showed how the spectral behavior is significantly different for the two cases, hence a more prominent turbulence is generated when the anisotropy develop within the nozzle is accounted for. The intricate phenomenas undergoing between atomization and turbulence do not have a fully physical explanation. 
Regardless, this thesis clearly highlights how higher level of turbulence (intended as $R e_{\lambda}$ ) corresponds to higher atomization. This hypothesis has been clearly proved both in changing the integral lengthscale $L$ in Chapter 5 and in using different turbulent structures incoming from the nozzle in Chapter 7 . Although it may seem a trivial conclusion, its effects are not. In fact, experimental measurements such as spray cone angle and penetration are heavily affected by the turbulence level and the total atomized mass. For example, the cases with higher turbulence level (case 2 in Chapter 5 and the MBC case in Chapter 7) show a more prominent opening, which will likely lead in the disperse region to a larger cone angle and to its earlier formation. Also, that leads to a shorter intact core length, which is also a measurable parameter (e.g. using optical connectivity).

For these reasons, while the boundary condition may be of secondary importance for comparison with statistical data, it is not for comparison with physical measurements that are typical of these applications. This makes the study of this cases particularly challenging, as it is difficult to have a good degree of certainty about the fidelity with which the nozzle flow is actually calculated.

Furthermore, having a reliable PDF of droplet size distribution in this region is, currently, almost impossible due to the high optical density of the medium in the dense region. Similar PDFs collected in downstream regions may be biased, as in the disperse region, phenomenas as secondary atomization and coalescence are predominant and the droplet size distribution is likely heavily affected by it. Furthermore, the description of the temporal generation of the droplets made in Section 7.7 shows how most of the smaller droplets are generated due to the turbulence created by the spray tip, hence this phenomena is likely to reduce in the disperse region (where the spray tip has less mass due to the atomization in the dense region) and be mitigated by coalescence.

Once again, the best boundary condition cannot be defined by this results, but it is mostly a matter concerning the available data for comparison. It is my personal opinion that the best way to settle this issue is working jointly between experimentalists and experts in designing experiments that can be faithfully replicated both using DNS and experimental techniques. This point will also be addressed later.

\subsubsection{Considerations over the analysis of droplets properties}

The main considerations performed over the atomization behavior, intended as both droplets properties (e.g. see the Klimontovich description Section 4.2) 
and their generation in time should be drawn by the analysis in Chapter 7 . The previous studies performed in Chapter 5, although qualitatively similar and extracted with the same methodology, do not present a sufficient collection of data and should be considered primarily as an introduction and design study, that ultimately led to the study in Chapter 7. Here, the droplet has been fully characterized by means of various analysis.

Regarding the droplets size distribution, some interesting patterns have been found. In a first place, it is interesting to notice that, as stated in previous section, the statistical behavior is not influenced by turbulence generated within the nozzle, as far as it is sufficient to generate a considerable number of breakup events (unlike case 0 in Chapter 5). On the other hand, the amount of droplets generated changes significantly with the turbulence developed within the nozzle, as showed in Chapter 7 by an increment of $20 \%$ in the total atomized mass of the MBC case, compared to the SBC. Both these informations give interesting insight on the physical process generating the atomization. In fact, it appears that, regardless of the total amount of mass that is breaking-up into droplets, the diameter distribution keeps a consistent behavior. In other words, the dynamic that dictates the diameter of the droplet is unrelated to the turbulence intensity level of the region or the number of breakup events occurring in the surroundings. This is quite an interesting and important result, that may lead to further more specific studies in future. It is the author opinion that a description of the breakup phenomena can be related to the way in which the energy distributes among different wavelength in the energy cascade. This may be further supported by the similarity in the power-law (log-normal) behavior that both the droplet distribution and the energy cascade display. The logical consequence would than be that the wavelengths (e.g. in the frequencies axis in the energy spectra) and their respective energy content are related to the droplet formation by means of physical scaling concerning the smaller scales. By derivation, it could also be stated that larger wavelengths are responsible for creating the most energetic surface perturbation, which are required by the liquid core for breaking up into smaller liquid structures. The energy of smaller frequencies will then sum up and then generate the breakup into a droplet of specific diameter, which can be related to the frequency that triggered it. Evidently, larger levels of turbulence imply more energy to be dissipated, hence a larger number of smaller structures developed, therefore a higher number of "triggered events" that cause the droplet breakup. This topic is obviously very vast and likely one of the most challenging in fluid mechanics, currently researched in many research groups worldwide. 
Some interesting insights have also been provided by the temporal description of spray formation, in Section 7.7. Here it is presented how smaller droplets are mostly created by the spray tip during the initial transient. This evidence is also supported by the axial development of the droplet size distribution presented in Section 7.6. This is a novel and important contribution for many reasons. In a first place, to the best of the author knowledge, the simulations performed in Chapter 7 are the longest and statistically most relevant studies available, as appears by literature analysis and conversations with peers. Despite that, both cases are still significantly shorter than the average duration of experiments (which are usually performed for at least a order of magnitude longer time). This leads to believe that the smaller droplets will have less influence for longer statistical averages, as their generation after the spray tip penetrates is marginal. As longer DNS simulations are currently extremely expansive (tens of millions core-hours), this bias in the droplet size distribution should be considered during comparison with experiments and while performing statistical description of the spray.

\subsubsection{Consideration on multiphase turbulence}

Turbulence is still one of the most challenging topic and its nature is still considered to be a widely unknown, despite all the latest findings (some of which, extremely recent as, for instance, Cardesa et al. [1] and Lanotte et al. [2]). Multiphase turbulent flows are even more complex, as less theoretical studies are available and there is a substantial lack of both formal theory and numerical/experimental evidences (at least compared with the single phase flow). This framework makes it difficult to operate a sound analysis of turbulence in atomizing sprays, lacking evidence and data support. Furthermore, the scientific community has not yet found, to the best of the author knowledge, a unique approach to tackle the topic, making it somehow difficult to navigate literature with consistent guidelines.

This thesis, while is not trying to make definitive statements, tries to add onto this issue, merging the gap left between the analysis of multiphase flows and the analysis of turbulence in single-phase flows. The reasons for this ambitious goal are many and will be discussed in the following.

In a first place, the author wish of providing some type of validation for the results obtained. It has been vastly pointed out, both in this thesis and in literature, that the lack of certainties in determining the smallest motion scale is somehow still a significant issue in these flows, often overlooked by researchers. This was the original motivation that moved towards the use of 
the pseudo-fluid approach, as a way to use the Kolmogorov scale to estimate the mesh quality and the reliability of the simulation results.

The analysis of the turbulent spectra and the autocorrelation, on the other hand, had multiple objectives. In a first place, once again, assessing the amount of unresolved energy in the simulation. The Kolmogorov scaling of the spectra showed in Section 6.4 aimed to estimate the differences and similarities, if any, with similar spectrum computed for single phase turbulence. The evidences produced in Chapter 6 seem to suggest that the atomization process do not affect significantly the flow spectra. Some of the deviations (e.g. at higher frequencies) displayed in Section 6.4 will be object of future studies that will require finer meshes. Furthermore, the usage of the pseudofluid approach proved once again to be an interesting tool, that allowed the comparison of single phase and multiphase flows thanks to the curve collapse obtained in the dimensionless spectra.

Another main point of the analysis of turbulence is the comparison of the single phase jet against the atomizing spray. The analogy between these cases has a long history and it proves to be an accurate comparison in the disperse region. On the other hand, the dense-region in sprays (or the near field in jets) is still vastly unknown for both cases. Regardless, single phase jets have a substantial amount of data, for spectra, one and two points statistics and axial evolution, while most of these data are lacking for sprays. The analysis provided in Chapter 7 showed how the self-similar behavior for the axial velocity is captured quite well. On the other hand, both the radial velocity component and the pulsating components of the velocity appear to be quite different, especially close to the spray centerline. This behavior has likely two reasons. In fact, a possible explanation is that the spray and jet are too different in nature, with the prominent presence of a high density flow in the centerline that significantly changes the momentum. Further studies will address this issue, trying to provide an explanation for the differences highlighted in Chapter 7.

\subsubsection{How far is DNS from reality?}

This may be one of the most important problems of DNS for engineering applications. In fact, the cases studied in this thesis are at moderate Reynolds (far from real applications) and even for such conditions, DNS simulations seem to not fully resolve the Kolmogorov scale. Furthermore, it is likely that the Kolmogorov scale is not even the smallest scale that need to be accounted for in atomizing flows, as flow interface thickness and atomization scales (such as Hinze scale) may imply the need of higher space resolution. 
While all these aspects are real problems that need to be taken into account, from an applied standpoint it is of fundamental importance to understand what can be done with modern computer capabilities, what may provide erroneous data and what are the next steps to undertake. As already pointed out at the beginning of this thesis, it is of fundamental importance to improve the understanding of the physical phenomenas behind primary atomization. In order to achieve that, a trade-off needs to be found between experimental and numerical approaches. Hence, in the author opinion, it is of fundamental importance to achieve reliable experiments of atomization that can be accurately reproduce numerically. The validation of the numerical simulations against experiments will allow to gain a significant amount of knowledge from simulations, and likely understand the phenomenas that may then be modeled in real applications. On the other hand, while this fundamental studies are ongoing, DNS may still be used for engineering applications, but it is important to keep in mind that results from under-resolved DNS may only be used to draw trends and qualitative analysis, while quantitative results as well as physical conclusions may still be vastly incorrect. Finally, for spray DNS simulations to reach the level of accuracy required for studying real injection conditions (especially in propulsion applications), a further step in supercomputing will be required, given both by the codes' improvements as well as HPC architectures improvement.

\subsection{Development of a post processing toolbox}

As already mentioned, the goal of this thesis is to provide insight on physical processes involved in sprays and primary atomization. For this reason, the toolboxes developed for this purpose were addressed only theoretically and qualitatively, but it is worth recompiling the tools developed. The main focuses in developing computational tools were on:

- Implement boundary conditions, both for the MBC and the SBC case.

- Storing and handling datasets.

- Planes parallel post-process.

- Domain parallel post-process.

- Improve ParisSimulator computational performances. 
To clarify the main contribution, each tool will be summarily described in the following section. All the tools are currently used by all students and faculty members involved in DNS of sprays in CMT-Motores Térmicos. Aside from the implemented tools in ParisSimulator, all the postprocessing and preprocessing tools were implemented in Python 2.7 and parallelized using MPI.

\subsubsection{Boundary conditions in ParisSimulator}

Both the boundary conditions have been implemented within the ParisSimulator code and parallelized using MPI. As the matrix describing the velocity fluctuations in the nozzle were quite reduced (larger case simulated used a stencil of $150^{3}$ elements for describing the nozzle in the SBC case) the faster way to implementing was handling the data processing (whether computing the fluctuations in the SBC or loading the data in the $\mathrm{MBC}$ ) on one core (usually called rank0) and redistribute it using the MPI protocol.

\subsubsection{Datasets storing and handling}

Data storage and handling is a often unsung issue in DNS, but the amount of data that a simulation can provide may be overwhelming, especially if statistical analysis are required. The original output implemented in ParisSimulator required approximately 140 bytes per cell, which translates into roughly $8.5 G B$ of storage required per snapshot on $60 \mathrm{M}$ cells simulations (like the ones in Chapter 6 ). The related postprocess may increase the data storage by a factor 4, requiring almost 4TB of data storage in VTK legacy 2.0 format. By using the VTK-XML compressed format, and switching to a rectilinear grid (instead of structured grid) representation this information can be reduced by $80 \%$. This reduction also reflects significantly in the post-process. VTKXML allows for significant advantages, like fully parallel visualization, high compression, high performance parallel I/O, but due to implementation and compilation issues in HPC devices, the whole transformation had to be done offline in post-processing. Furthermore, the number of file can be overwhelming (millions of files, especially for the planes), and this is a significant issue in handling files, both in transfering to/from HPC clusters or for post process procedures.

A solution to this problem came recently thanks to the H5 output format (implemented in the DNS research group at CMT-Motores Térmicos) where a MPI I/O is dumping all data chunks into a single file. This solution is the one currently implemented in ParisSimulator and available to all the code users worldwide. 


\subsubsection{Parallel post-process}

The parallel post-process was developed for two main reasons. In a first place, it is necessary to speed up the data analysis, which for big datasets may easily takes months. Secondly, process independent chunks of data is a necessity when the dataset is significantly larger than the the amount of memory available in workstations (in many cases by at least a order of magnitude). In fact, latest simulation performed within the research group reached up to 2 billions of cells, requiring hundreds of GB just for opening a single snapshot.

The whole environment was developed so that both the analysis of planes and total domain can be performed in individual chunks, bringing to zero the interprocessor communication while limiting the amount of memory per core requires. For example, for the planes analysis, sets of planes are attributed to each processor and generate partial outputs, which are finally recomposed by one processor off-line. In a similar fashion, the data for the whole domain is parallelized using regular sub-domain parallelization. This allows these programs to be executed both on workstations and supercomputer efficiently. An obvious downside of this approach is the high $\mathrm{I} / \mathrm{O}$ flux of data, but it is unavoidable to the best of the author knowledge.

\subsubsection{ParisSimulator performance improvement using GPU}

Using GPUs for scientific computing is a common trend nowadays and it is establishing as the future path towards which CFD for massive parallel application will need to steer. Both for historical reasons (many codes are adaptation of programs developed more than 20 years ago) and scientific gap (engineers and physicist are usually not academically prepared for computer science and vice versa) the GPUs usage is somewhat rare in the CFD world.

In this thesis, in collaboration with Institut Jean Le Rond d'Alembert at Sorbonne University (Paris), a GPU Poisson solver has been developed, as presented in Section 2.5. This feature allows the code ParisSimulator to operate on hybrid CPU/GPU clusters, which are the current state of the art architectures. This solver is particularly useful when in high gradients flows, like, for example, atomization with high density ratio. In water-air atomization, this solver has proved to speedup the simulation by a factor 3 in simple tests (e.g. 8CPUs against 8CPUs plus a commercial graphic card) and, for this reason, its development will be improved in the future. This feature also makes, to the best of the author knowledge, the code ParisSimulator one of the first multiphase flows DNS code able to perform on hybrid clusters such as the ones reported in the TOP500 list. 


\subsection{Future works}

As often remarked along this thesis, many interesting point have been raised that may legitimate future researches, works and collaborations. While some of those suggestions have yet to find a practical way to be implemented into a research and have only be theoretically discussed, others have actually been already planned and/or are already ongoing. In the interest of clarity each future activity will be specifically addressed in the following subsections.

\subsubsection{Using X-Rays and DNS for studying sprays}

This activity has been developed in collaboration with Argonne National Laboratories and CMT-Motores Térmicos. The aim of this study is comparing X-Rays simulations with DNS results, in order to validate the numerical approach and gain physical insight on the process and the data obtained by means of X-Rays measurements in the dense region. A key aspect for the comparison is recreating an experimental setup that can be simulated with a high degree of confidence on the uncertainties and the variabilities that may be encountered. At the current stage, both teams are working on designing an injection system that may be able to reproduce a pipe flow for the nozzle, hence been reproduced in LES/DNS simulation of the nozzle and compared against literature data.

\subsubsection{Analysis of Reynolds, Weber and density ratio influence over the atomization process}

This is an internal activity in CMT-Motores Térmicos and has been ongoing since 2018. The goal of this research project is using the toolbox developed in this thesis to analyze the influence of dimensionless numbers on the atomization process, while analyzing both the droplet generation and the turbulence statistics. Due to the high Reynolds number set as a goal in this project (about $\left.R e_{b}=15000\right)$ and the requirements in terms of Kolmogorov scale proved in this thesis, some simulations required for this project will require several millions of core-hours of computational time. Some simulations have already been partially completed, but more simulation time is required in order to achieve significant statistics and meaningful physical results. All the cases of this project will use the MBC feature implemented in ParisSimulator in this thesis. Finally, a near field visualization experimental comparison is also envisioned, due to a specific vessel designed and manufactured during this thesis in order to achieve this goal. The title of the corresponding national project is Estudio de la atomizacion mediante simulaciones DNS y tecnicas opticas de 
muy alta resolucion (ref.:RTI2018-099706-B-I00, supported by Ministerio de Ciencia, Innovación y Universidades)

\subsubsection{Analysis of aeroengines airblast atomizers using DNS}

This research is related to a European project, namely the CLEANSKYH2020. The goal is to provide a phenomenological atomization model for airblast atomizers in aeronautic applications, based on the results of a DNS simulations batch performed using ParisSimulator. The characterization of droplet size distribution performed in Chapter 7 will be extended to various injection condition in order to extract a predictive model able to complement the RANS/LES informations that are produced at an industrial level in the aeronautic sector.

\subsubsection{Analysis of atomization causality in relation to turbu- lence scale}

This project in not currently undergoing and aims to address one of the fundamental issues about primary atomization. The overall goal is to try relating turbulent structures and droplet formation by analyzing the effects caused by every specific turbulence frequency.

\section{References}

[1] Cardesa, José I., Vela-Martín, Alberto, and Jiménez, Javier. "The turbulent cascade in five dimensions". In: Science 357.6353 (2017), pp. 782-784. DOI: $10.1126 /$ science.aan7933.

[2] Lanotte, Alessandra S., Benzi, Roberto, Malapaka, Shiva K., Toschi, Federico, and Biferale, Luca. "Turbulence on a Fractal Fourier Set". In: Physical Review Letters 115.26 (2015), pp. 1-5. DOI: 10.1103 / PhysRevLett.115.264502. 

Appendices 



\section{Appendix A}

\section{GPU Poisson-Solver}

A GPU based solver is also available for solving the Poisson equation when a significant number of iterations is required to achieve convergence. The pressure is solved using a Jacobi method for the pressure iteration:

$$
\begin{aligned}
p_{i, j, k} & \leftarrow(1-\varpi) p_{i, j, k}+\frac{\varpi}{A_{7, i, j, k}}\left(A_{1, i, j, k} p_{i-1, j, k}\right. \\
& +A_{2, i, j, k} p_{i+1, j, k}+A_{3, i, j, k} p_{i, j-1, k}+A_{4, i, j, k} p_{i, j+1, k} \\
& \left.+A_{5, i, j, k} p_{i, j, k+1}+A_{6, i, j, k} p_{i, j, k-1}-A_{8, i, j, k}\right)
\end{aligned}
$$

Which is the three dimensional discretized form for Equation (2.11), where the pressure coefficient for the solution of the projection step are $A_{m, i, j, k}$ with $m$ being the element of the pressure stencil. The need for the Jacobi method instead of a Gauss-Seidel arises because of the intrinsic nature of GPU devices. The usual domain decomposition parallelization allows the implementation of the iterative step by using a simple for loop over the indexes $i, j, k$. The sequentiality of the indexes cannot be achieved on GPU devices, as in this case each index combination is ideally computed simultaneously. In this sense, the higher number of iteration required by the Jacobi method is mitigated by the speed-up provided by GPUs.

The memory handling is a critical aspect in GPUs applications and it is even more critical in DNS. Although a Jacobi method intrinsically requires doubling the memory usage for the matrix $p$, it also enables the leanest data 
transfer between CPUs and GPUs, which is also a critical aspect of normal CUDA applications. A Gauss-Seidel red-black solver is in principle possible and beneficial for certain applications. In fact, let us assume that the size of the matrix $p$ is $N_{p}=n_{x} n_{y} n_{z}$, the normal implementation of a red-black Gauss-Seidel solver would be

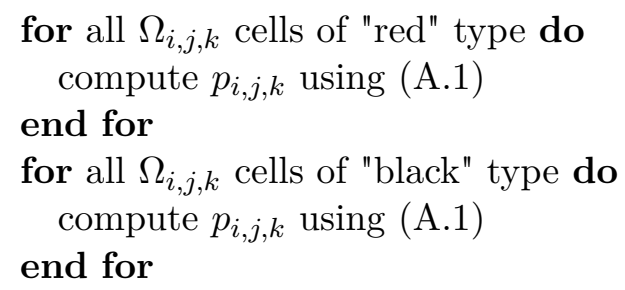

which inherently reduces the memory usage required. On the other hand, the first for loop is not parallelizable in an efficient way in $C U D A$. Therefore, such an algorithm would be beneficial from a memory standpoint, but would improve the computational time only if $N_{p}$ is at least 4 times greater than the number of GPU process available. As it is usually not the case, the beneficial effects of a red-black algorithm are limited, although it will be object of future studies.

The implementation of the algorithm is achieved by means of the opensource $C U D A$ library for $C$ developed by NVIDIA, while the intercommunication between processors is still achieved by using MPI. For this reason, an interface between Fortran90 and $C$ is created in module_CUDA.f90. By passing through the interface, each process transfers the matrices $A$ and $p$ to $C / C U D A$ environment (in poissonCUDA.cu) where the iteration step is performed. The boundary conditions, as well as the MPI communication, are enforced in the environment that originally created the MPI communication, hence these functions are programmed in cudaFun.f90. 


\section{Global Bibliography}

"A sub-grid surface dynamics model for sub-filter surface tension induced interface dynamics". In: Computers and Fluids 87.480 (2013), pp. 92-101. DOI: $10.1016 / j$.compfluid.2013.02.008 (cited on pages 30,31,33).

Afkhami, S. and Bussmann, M. "Height functions for applying contact angles to 3D VOF simulations". In: International Journal for Numerical Methods in Fluids 61.8 (2009), pp. 827-847. DOI: $10.1002 / \mathrm{fld} .1974$ (cited on page 60).

Agbaglah, Gilou et al. "Simulation parallèle adaptative octree d'écoulements multiphasiques par suivi d'interface de type volume de fluide". In: Comptes Rendus - Mecanique 339.2-3 (2011), pp. 194-207. DOI: 10.1016/j.crme. 2010.12.006 (cited on page 44).

Álamo, Juan C. del and Jiménez, Javier. "Spectra of the very large anisotropic scales in turbulent channels". In: Physics of Fluids 15.6 (2003), pp. 41-44. DOI: 10.1063/1.1570830 (cited on page 160).

Antonia, R. A., Anselmet, F., and Chambers, A. J. "Assessment of local isotropy using measurements in a turbulent plane jet". In: Journal of Fluid Mechanics 163.-1 (1986), pp. 365-391. DOI: 10.1017/S0022112086002331 (cited on page 98 ).

Ashgriz, Nasser. Handbook of Atomization and Sprays. Springer Science \& Business Media, 2011. DOI: 10 . 1007/978-1-4419-7264-4 (cited on pages 3, 21, 86, 129).

Balachandar, S. and Eaton, John K. "Turbulent Dispersed Multiphase Flow". In: Annual Review of Fluid Mechanics 42.1 (2010), pp. 111-133. DOI: 10. 1146/annurev.fluid.010908.165243 (cited on page 22). 
Balarac, Guillaume and Métais, Olivier. "The near field of coaxial jets: A numerical study". In: Physics of Fluids 17.6 (2005), pp. 1-14. DOI: 10. 1063/1.1900786 (cited on page 30).

Bassenne, Maxime, Esmaily, Mahdi, Livescu, Daniel, Moin, Parviz, and Urzay, Javier. "A dynamic spectrally enriched subgrid-scale model for preferential concentration in particle-laden turbulence". In: International Journal of Multiphase Flow 116 (2019), pp. 270-280. DOI: $10.1016 / \mathrm{j}$. ijmultiphaseflow.2019.04.025 (cited on page 149).

Bassenne, Maxime, Moin, Parviz, and Urzay, Javier. "Wavelet multiresolution analysis of particle-laden turbulence". In: Physical Review Fluids 3.8 (2018), p. 084304. DOI: 10.1103 / PhysRevFluids . 3.084304 (cited on page 32).

Batchelor, G. K., Howells, I. D., and Townsend, A. A. "Small-scale variation of convected quantities like temperature in turbulent fluid: Part 2. The case of large conductivity". In: Journal of Fluid Mechanics 5.1 (1959), pp. 134139. DOI: $10.1017 /$ S0022112059000106 (cited on page 28).

Battistoni, Michele et al. "Experimental and Computational Investigation of Subcritical Near-Nozzle Spray Structure and Primary Atomization in the Engine Combustion Network Spray D". In: SAE Technical Paper 2018-010277 (2018), pp. 1-15. DOI: 10.4271/2018-01-0277 (cited on pages 17, 19).

Bell, John B, Colella, Phillip, and Glaz, Harland M. "In this paper we develop a second-order projection method for the incom- pressible Navier-Stokes equations". In: Journal of Computational Physics 283 (1989), pp. 257-283 (cited on page 50).

Benajes, Jesus, Salvador, Francisco Javier, Carreres, Marcos, and Jaramillo, David. "On the relation between the external structure and the internal characteristics in the near-nozzle field of diesel sprays". In: Proceedings of the Institution of Mechanical Engineers, Part D: Journal of Automobile Engineering 231.3 (2017), pp. 360-371. DOI: 10.1177/0954407016639464 (cited on pages 113, 175, 179, 184).

Berrocal, E., Kristensson, E., Hottenbach, P., Aldén, M., and Grünefeld, G. "Quantitative imaging of a non-combusting diesel spray using structured laser illumination planar imaging". In: Applied Physics B: Lasers and Optics 109.4 (2012), pp. 683-694. DOI: 10.1007/s00340-012-5237-9 (cited on page 11). 
Berrocal, Edouard, Kristensson, Elias, Richter, Mattias, Linne, Mark, and Alden, Marcus. "Multiple Scattering Suppression in Planar Laser Imaging of Dense Sprays By Means of Structured Illumination". In: Atomization and Sprays 20.2 (2010), pp. 133-139. DOI: 10.1615/atomizspr .v20.i2. 30 (cited on page 11).

Berrocal, Edouard, Sedarsky, David L., Paciaroni, Megan E., Meglinski, Igor V., and Linne, Mark A. "Laser light scattering in turbid media Part I: Experimental and simulated results for the spatial intensity distribution". In: Optics Express 15.17 (2007), p. 10649. DOI: 10.1364/oe.15.010649 (cited on page 8).

Bnà, S., Manservisi, S., Scardovelli, R., Yecko, P., and Zaleski, S. "Vofi - A library to initialize the volume fraction scalar field". In: Computer Physics Communications 200 (2016), pp. 291-299. DOI: 10.1016/j.cpc.2015.10. 026 (cited on page 114).

Bourouiba, Lydia, Dehandschoewercker, Eline, and Bush, John W.M. "Violent expiratory events: On coughing and sneezing". In: Journal of Fluid Mechanics 745 (2014), pp. 537-563. DOI: 10.1017/jfm. 2014.88 (cited on page 1).

Brackbill, J. U., Kothe, D. B., and Zemach, C. "A continuum method for modeling surface tension". In: Journal of Computational Physics 100.2 (1992), pp. 335-354. DOI: 10 . 1016/0021-9991(92) 90240-Y (cited on pages 58,59$)$.

Bravin, Alberto. "Exploiting the x-ray refraction contrast with an analyser: The state of the art". In: Journal of Physics D: Applied Physics 36.10 A (2003). DOI: 10.1088/0022-3727/36/10A/306 (cited on page 15).

Briggs, William L., Henson, Van Emden, and McCormick, Steve F. "A Multigrid Tutorial, Second Edition". In: A Multigrid Tutorial, Second Edition December 2015 (2011). DOI: 10.1137/1.9780898719505 (cited on page 53).

Burton, Tristan M. and Eaton, John K. "Fully resolved simulations of particleturbulence interaction". In: Journal of Fluid Mechanics 545.2005 (2005), pp. 67-111. DOI: 10.1017/S0022112005006889 (cited on page 169).

Canu, Romain et al. "Where does the droplet size distribution come from?" In: International Journal of Multiphase Flow 107.January 2019 (2018), pp. 230-245. DOI: 10.1016/j.ijmultiphaseflow.2018.06.010 (cited on pages 22, 23). 
Cardesa, José I., Vela-Martín, Alberto, and Jiménez, Javier. "The turbulent cascade in five dimensions". In: Science 357.6353 (2017), pp. 782-784. DOI: 10.1126/science. aan7933 (cited on page 193).

Charalampous, G., Hardalupas, Y., and K. P. Taylor, A. M. "Novel Technique for Measurements of Continuous Liquid Jet Core in an Atomizer". In: AIAA Journal 47.11 (2009), pp. 2605-2615. DOI: 10.2514/1.40038 (cited on pages 11,13$)$.

Chesnel, J., Menard, Thibaut, Reveillon, Julien, and Demoulin, FrancoisXavier. "Subgrid analysis of liquid jet atomization". In: Atomization and Sprays 21.1 (2011), pp. 41-67. DOI: 10.1615/atomizspr.v21.i1.40 (cited on page 93$)$.

Chorin, Alexandre Joel. "Numerical solution of the Navier-Stokes equations". In: Mathematics of Computation 22 (1968), pp. 745-762. DOI: 10.2307/ 2004575 (cited on page 48).

Comte-Bellot, Geneviève and Corrsin, Stanley. "Simple Eulerian time correlation of full-and narrow-band velocity signals in grid-generated, âisotropic' turbulence". In: Journal of Fluid Mechanics 48.2 (1971), pp. 273-337. DOI: 10.1017/S0022112071001599 (cited on pages 153, 154).

CORIA. Archer code. 2005 (cited on page 45).

Corrsin. Spectra and Diffusion in a Round Turbulent Jet. Tech. rep. 8. 1943, pp. 399-405 (cited on pages 5, 98, 164).

Cotton, William R., Bryan, George, and Heever, Susan C. van den. Turbulence. Vol. 99. C. Cambridge University Press, 2011, pp. 53-86. DOI: 10.1016/ S0074-6142(10)09909-2 (cited on pages 88-90, 153).

Craske, John, Debugne, Antoine L.R., and Van Reeuwijk, Maarten. "Shearflow dispersion in turbulent jets". In: Journal of Fluid Mechanics 781 (2015), pp. 28-51. DOI: 10.1017/jfm.2015.417 (cited on page 98).

Crua, Cyril, Heikal, Morgan R., and Gold, Martin R. "Microscopic imaging of the initial stage of diesel spray formation". In: Fuel 157 (2015), pp. 140150. DOI: $10.1016 / \mathrm{j}$.fuel.2015.04.041 (cited on pages 13,14).

Davies, T. V. and Batchelor, G. K. The Theory of Homogeneous Turbulence. Vol. 38. 323. Cambridge university press, 2007, p. 64. DOI: 10.2307 / 3609796 (cited on page 70). 
Desantes, J. M., Salvador, F. J., López, J. J., and De La Morena, J. "Study of mass and momentum transfer in diesel sprays based on X-ray mass distribution measurements and on a theoretical derivation". In: Experiments in Fluids 50.2 (2011), pp. 233-246. DOI: $10.1007 / \mathrm{s} 00348-010-0919-8$ (cited on page 134).

Desjardins, Olivier. NGA. 2012 (cited on page 44).

Desjardins, Olivier, McCaslin, Jeremy, Owkes, Mark, and Brady, Peter. "Direct Numerical and Large-Eddy Simulation of Primary Atomization in Complex Geometries". In: Atomization and Sprays 23.11 (2013), pp. 10011048. DOI: 10.1615/AtomizSpr.2013007679 (cited on pages 3, 26, 96).

Desjardins, Olivier, Moureau, Vincent, and Pitsch, Heinz. "An accurate conservative level set/ghost fluid method for simulating turbulent atomization". In: Journal of Computational Physics 227.18 (2008), pp. 8395-8416. DOI: $10.1016 / \mathrm{j} \cdot \mathrm{jcp} .2008 .05 .027$ (cited on pages 26, 44).

Druault, P. et al. "Generation of Three-Dimensional Turbulent Inlet Conditions for Large-Eddy Simulation". In: AIAA Journal 42.3 (2008), pp. 447456. DOI: 10.2514/1.3946 (cited on page 68).

Du, Wei, Bao, Xiaojun, Xu, Jian, and Wei, Weisheng. "Computational fluid dynamics (CFD) modeling of spouted bed: Influence of frictional stress, maximum packing limit and coefficient of restitution of particles". In: Chemical Engineering Science 61.14 (2006), pp. 4558-4570. DOI: 10.1016/ j.ces.2006.02.028 (cited on page 102).

Dukowicz, John K. "A particle-fluid numerical model for liquid sprays". In: Journal of Computational Physics 35.2 (1980), pp. 229-253. DOI: 10.1016/ 0021-9991 (80)90087-X (cited on page 104).

Duran, S. P., Porter, J. M., and Parker, T. E. "Ballistic Imaging of Sprays at Diesel Relevant Conditions". In: 12th International Conference on Liquid Atomization and Spray Systems. 2012, pp. 1-5 (cited on page 10).

Duret, B., Reveillon, J., Menard, T., and Demoulin, F. X. "Improving primary atomization modeling through DNS of two-phase flows". In: International Journal of Multiphase Flow 55 (2013), pp. 130-137. DOI: 10 . 1016/ j . ijmultiphaseflow.2013.05.004 (cited on page 22).

Duret, B. et al. "DNS analysis of turbulent mixing in two-phase flows". In: International Journal of Multiphase Flow 40 (2012), pp. 93-105. DOI: 10. 1016/j.ijmultiphaseflow. 2011.11.014 (cited on pages 92, 93, 102, 148). 
Eastwood, C. D., Armi, L., and Lasheras, J. C. "The breakup of immiscible fluids in turbulent flows". In: Journal of Fluid Mechanics 502 (2004), pp. 309-333. DOI: 10.1017/S0022112003007730 (cited on page 87).

Eaton, J. K. and Fessler, J. R. "Preferential concentration of particles by turbulence". In: International Journal of Multiphase Flow 20.SUPPL. 1 (1994), pp. 169-209. DOI: 10 .1016/0301-9322(94) 90072-8 (cited on page 92$)$.

ECN. ECN Webpage (cited on page 7).

Eggels, J. G. M. et al. "Fully developed turbulent pipe flow: a comparison between direct numerical simulation and experiment". In: Journal of Fluid Mechanics 268.-1 (2006), p. 175. DOI: 10.1017/S002211209400131X (cited on pages $71-75)$.

El Khoury, George K. et al. "Direct numerical simulation of turbulent pipe flow at moderately high reynolds numbers". In: Flow, Turbulence and Combustion 91.3 (2013), pp. 475-495. DOI: 10.1007/s10494-013-9482-8 (cited on pages 72,74$)$.

Elghobashi, Said. "Direct Numerical Simulation of Turbulent Flows Laden with Droplets or Bubbles". In: Annual Review of Fluid Mechanics 51.1 (2018), pp. 217-244. DOI: 10 . 1146 / annurev-fluid-010518-040401 (cited on pages 22, 92, 149).

Fedkiw, Ronald P., Aslam, Tariq, Merriman, Barry, and Osher, Stanley. "A Non-oscillatory Eulerian Approach to Interfaces in Multimaterial Flows (the Ghost Fluid Method)". In: Journal of Computational Physics 152.2 (1999), pp. 457-492. DOI: 10.1006/jcph.1999.6236 (cited on page 59).

Fellouah, H., Ball, C. G., and Pollard, A. "Reynolds number effects within the development region of a turbulent round free jet". In: International Journal of Heat and Mass Transfer 52.17-18 (2009), pp. 3943-3954. DOI: 10.1016/j.ijheatmasstransfer.2009.03.029 (cited on page 30).

Fuster, Daniel and Popinet, Stéphane. "An all-Mach method for the simulation of bubble dynamics problems in the presence of surface tension". In: Journal of Computational Physics 374 (2018), pp. 752-768. DOI: 10.1016/ j.jcp.2018.07.055 (cited on page 44).

G, Charalampous, C, Hadjiyiannis, Y, Hardalupas, and A, Taylor. "Measurement of continuous liquid jet length in atomizers with optical connectivity, electrical conductivity and high-speed photography techniques". In: ... on Liquid ... September. 2010, pp. 1-10 (cited on pages 6, 11). 
Gauding, M., Danaila, L., and Varea, E. "One-point and two-point statistics of homogeneous isotropic decaying turbulence with variable viscosity". In: International Journal of Heat and Fluid Flow 72.May (2018), pp. 143-150. DOI: $10.1016 /$ j.ijheatfluidflow. 2018.05.013 (cited on pages 90, 148).

Ghiji, M., Goldsworthy, L., Brandner, P. A., Garaniya, V., and Hield, P. "Numerical and experimental investigation of early stage diesel sprays". In: Fuel 175 (2016), pp. 274-286. DOI: 10.1016/j.fuel.2016.02.040 (cited on page 113 ).

Gorokhovski, M. A. and Saveliev, V. L. "Analyses of Kolmogorov's model of breakup and its application into Lagrangian computation of liquid sprays under air-blast atomization". In: Physics of Fluids 15.1 (2003), pp. 184192. DOI: 10.1063/1.1527914 (cited on pages 31,32).

Gorokhovski, Mikhael and Herrmann, Marcus. "Modeling Primary Atomization". In: Annual Review of Fluid Mechanics 40.1 (2008), pp. 343-366. DOI: 10.1146/annurev.fluid.40.111406.102200 (cited on pages 6, 7, 28, 32, $46,86,152,155,173)$.

Grinstein, F. F. Large Eddy Simulation for Incompressible Flows, An Introduction, 3rd Edition. Vol. 46. 4. Springer Science \& Business Media, 2008, pp. 1021-1023. DOI: 10.2514/1.36015 (cited on page 68).

Gureyev, T. E. et al. "Refracting Röntgen's rays: Propagation-based x-ray phase contrast for biomedical imaging". In: Journal of Applied Physics 105.10 (2009). DOI: 10.1063/1.3115402 (cited on page 15).

Harris, V. G., Graham, J. A.H., and Corrsin, S. "Further experiments in nearly homogeneous turbulent shear flow". In: Journal of Fluid Mechanics 81.4 (1977), pp. 657-687. DOI: 10 . 1017 / S0022112077002286 (cited on pages 153,154$)$.

Hasslberger, Josef, Ketterl, Sebastian, Klein, Markus, and Chakraborty, Nilanjan. "Flow topologies in primary atomization of liquid jets: A direct numerical simulation analysis". In: Journal of Fluid Mechanics 859 (2019), pp. 819-838. DOI: 10.1017/ jfm. 2018 . 845 (cited on pages 30, 45, 113, $152)$.

Herrmann, M. "A parallel Eulerian interface tracking/Lagrangian point particle multi-scale coupling procedure". In: Journal of Computational Physics 229.3 (2010), pp. 745-759. DOI: $10.1016 /$ j.jcp. 2009.10 .009 (cited on pages 95,104$)$. 
Hinze, J. O. and Shell-laboratory, Royal Dutch. "Fundamentals of the hydrodynamic mechanism of splitting in dispersion processes". In: AIChE Journal 1.3 (1955), pp. 289-295. DOI: 10.1002/aic.690010303 (cited on page 28).

Hirt, C. W. and Nichols, B. D. "Volume of fluid (VOF) method for the dynamics of free boundaries". In: Journal of Computational Physics 39.1 (1981), pp. 201-225. DOI: 10.1016/0021-9991 (81) 90145-5 (cited on page 57).

Hirt, C.W W and Nichols, B.D D. "Volume of fluid (VOF) method for the dynamics of free boundaries". In: Journal of Computational Physics 39.1 (1981), pp. 201-225. DOI: 10 . 1016/0021-9991(81) 90145-5 (cited on page 56).

Hoepffner, J., Naka, Y., and Fukagata, K. "Realizing turbulent statistics". In: Journal of Fluid Mechanics 676 (2011), pp. 54-80. DOI: 10.1017 / jfm . 2011.32 (cited on page 68).

Hoyas, Sergio and Jiménez, Javier. "Scaling of the velocity fluctuations in turbulent channels up to $\operatorname{Re} \tau=2003$ ". In: Physics of Fluids 18.1 (2006), p. 011702. DOI: 10.1063/1.2162185 (cited on page 74).

Hult, J. et al. "Interior flow and near-nozzle spray development in a marineengine diesel fuel injector". In: Experiments in Fluids 57.4 (2016), p. 49. DOI: $10.1007 / \mathrm{s} 00348-016-2134-8$ (cited on page 113).

Hussein J. Hussein, Steven P. Capps, and William K. George. "Velocity measurements in a high-Reynolds-number, momentum-conserving, axisymmetric, turbulent jet". In: Journal of Fluid Mechanics 001.258 (1994), pp. 3175 (cited on pages 98, 134, 161, 162, 173).

Ilavsky, Jan and Jemian, Peter R. "Irena: Tool suite for modeling and analysis of small-angle scattering". In: Journal of Applied Crystallography 42.2 (2009), pp. 347-353. DOI: $10.1107 /$ S0021889809002222 (cited on page 17).

Issa, R. I. "Solution of the implicitly discretised fluid flow equations by operator-splitting". In: Journal of Computational Physics 62.1 (1986), pp. 40-65. DOI: 10.1016/0021-9991 (86)90099-9 (cited on page 74).

Jarrahbashi, D. and Sirignano, W. A. "Invited Article: Vorticity dynamics for transient high-pressure liquid injection". In: Physics of Fluids 26.10 (2014). DOI: 10.1063/1.4895781 (cited on pages 24, 25, 30, 31, 126).

Jarrahbashi, D. and Sirignano, W. A. "Invited Article: Vorticity dynamics for transient high-pressure liquid injection". In: Physics of Fluids 26.10 (2014). DOI: 10.1063/1.4895781 (cited on page 169). 
Jarrahbashi, D., Sirignano, W. A., Popov, P. P., and Hussain, F. "Early spray development at high gas density: hole, ligament and bridge formations". In: Journal of Fluid Mechanics 792 (2016), pp. 186-231. DOI: 10.1017/ jfm.2016.71 (cited on pages 25, 45, 68, 126, 127, 169).

Jeong, Jinhee, Hussain, Fazle, Jinhee, Jeong, and Fazle, Hussain. "On the identification of a vortex". In: Journal of Fluid Mechanics 285.October (1995), pp. 69-94. DOI: doi : 10.1017/S0022112095000462 (cited on pages 76, 77).

Jimenez, Javier. "Turbulent velocity fluctuations need not be Gaussian". In: Journal of Fluid Mechanics 376.-1 (2000), pp. 139-147 (cited on page 146).

Kaiser, Max, Heilig, Ansgar, and Dinkelacker, Friedrich. "Application of the Optical Connectivity Method to a Real Size Heavy Duty CIDI-Injector ( Application of the Optical Connectivity Method )". In: COMODIA 2012. Comodia. 2012, pp. 506-511 (cited on page 12).

Kastengren, A. et al. "Measurements of droplet size in shear-driven atomization using ultra-small angle x-ray scattering". In: International Journal of Multiphase Flow 92 (2017), pp. 131-139. DOI: $10.1016 / \mathrm{j}$. ijmultiphaseflow.2017.03.005 (cited on page 17).

Kastengren, Alan and Powell, Christopher F. "Synchrotron X-ray techniques for fluid dynamics". In: Experiments in Fluids 55.3 (2014). DOI: 10.1007/ s00348-014-1686-8 (cited on pages 6, 15, 17).

Kastengren, Alan et al. "Correlation of Split-Injection Needle Lift and Spray Structure". In: SAE Technical Paper Series 1 (2011). DOI: 10.4271/201101-0383 (cited on pages 17, 18).

Keating, Anthony, Piomelli, Ugo, Balaras, Elias, and Kaltenbach, Hans Jakob. "A priori and a posteriori tests of inflow conditions for large-eddy simulation". In: Physics of Fluids 16.12 (2004), pp. 4696-4712. DOI: 10.1063/1. 1811672 (cited on page 30).

Kim, J. and Moin, P. "Application of a fractional-step method to incompressible Navier-Stokes equations". In: Journal of Computational Physics 59.2 (1985), pp. 308-323. DOI: 10 .1016/0021-9991(85) 90148-2 (cited on page 49).

Kim, K. C. and Adrian, R. J. "Very large-scale motion in the outer layer". In: Physics of Fluids 11.2 (1999), pp. 417-422. DOI: 10.1063/1.869889 (cited on page 74 ). 
Klein, M., Sadiki, A., and Janicka, J. "A digital filter based generation of inflow data for spatially developing direct numerical or large eddy simulations". In: Journal of Computational Physics 186.2 (2003), pp. 652-665. DOI: 10. 1016/S0021-9991(03)00090-1 (cited on pages 22, 33, 67-71, 113).

Kolmogorov, A. "On the log-normal distribution of particles sizes during breakup process". In: Dokl. Akad. Nauk. XXXI.4 (1941), pp. 99-101 (cited on pages $31,32,86)$.

Kolmogorov, A. N. "The Local Structure of Turbulence in Incompressible Viscous Fluid for Very Large Reynolds Numbers". In: Proceedings of the Royal Society A: Mathematical, Physical and Engineering Sciences 434.1890 (1991), pp. 9-13. DOI: 10.1098/rspa . 1991.0075 (cited on pages $28,31,76,86-88)$.

Kristensson, E., Berrocal, E., Richter, M., Pettersson, S.-G., and Aldén, M. "High-speed structured planar laser illumination for contrast improvement of two-phase flow images". In: Optics Letters 33.23 (2008), p. 2752. DOI: 10.1364/ol.33.002752 (cited on page 11).

Lanotte, Alessandra S., Benzi, Roberto, Malapaka, Shiva K., Toschi, Federico, and Biferale, Luca. "Turbulence on a Fractal Fourier Set". In: Physical Review Letters 115.26 (2015), pp. 1-5. DOI: 10.1103/PhysRevLett.115. 264502 (cited on page 193).

Lasheras, J. C., Eastwood, C., Martínez-Bazán, C., and Montaes, J. L. "A review of statistical models for the break-up an immiscible fluid immersed into a fully developed turbulent flow". In: International Journal of Multiphase Flow 28.2 (2002), pp. 247-278. DOI: 10.1016/S0301-9322(01) 00046-5 (cited on page 87).

Lasheras, J. C. and Hopfinger, E. J. "Liquid Jet instability and Atomization in a Coaxial Gas Stream". In: Annu. Rev. Fluid Mech. 32.1 (2000), pp. 275308. DOI: 10.1146/annurev.fluid.32.1.275 (cited on page 1).

Lebas, R., Menard, T., Beau, P.A., Berlemont, A., and Demoulin, FrançoisXavier. "Numerical simulation of primary break-up and atomization: DNS and modelling study." In: International Journal of Multiphase Flow 35.3 (2009), pp. 247-260. DOI: 10.1016/j.ijmultiphaseflow.2008.11.005 (cited on pages 22, 68, 69, 71, 113, 114, 152).

Lee, Sangsan, Lele, Sanjiva K., and Moin, Parviz. "Simulation of spatially evolving turbulence and the applicability of Taylor's hypothesis in compressible flow". In: Physics of Fluids A 4.7 (1992), pp. 1521-1530. DOI: 10.1063/1.858425 (cited on page 68). 
Leer, Bram van and Powell, Kenneth G. Introduction to Computational Fluid Dynamics. 2010. DOI: 10 . 1002/9780470686652 . eae048 (cited on pages 51-53).

Lefebvre, A. and Mcdonell, Vincent. Atomization and Sprays, Second Edition. CRC Press, 2017, p. 300. DOI: 10.1016/0009-2509(90)87140-N (cited on pages 4,21$)$.

Leonard, B. P. "A stable and accurate convective modelling procedure based on quadratic upstream interpolation". In: Computer Methods in Applied Mechanics and Engineering 19.1 (1979), pp. 59-98. DOI: 10.1016/00457825 (79) 90034-3 (cited on page 50).

Lin, Shengxiang, Lu, Jiacai, Tryggvason, Gretar, and Zhang, Ying. "A numerical study of oscillation induced coalescence in bubbly flows". In: Physics of Fluids 30.12 (2018). DOI: 10.1063/1.5059558 (cited on page 54).

Ling, Y., Fuster, D., Tryggvason, G., and Zaleski, S. "A two-phase mixing layer between parallel gas and liquid streams: Multiphase turbulence statistics and influence of interfacial instability". In: Journal of Fluid Mechanics 859 (2019), pp. 268-307. DOI: 10.1017/jfm.2018.825 (cited on pages 26, 28, $45,78,87,94-96,148)$.

Ling, Y., Zaleski, S., and Scardovelli, R. "Multiscale simulation of atomization with small droplets represented by a Lagrangian point-particle model". In: International Journal of Multiphase Flow 76 (2015), pp. 122-143. DOI: 10.1016/j.ijmultiphaseflow.2015.07.002 (cited on pages 26, 69, 95, 104).

Ling, Yue, Fuster, Daniel, Zaleski, Stéphane, and Tryggvason, Grétar. "Spray formation in a quasiplanar gas-liquid mixing layer at moderate density ratios: A numerical closeup". In: Physical Review Fluids 2.1 (2017), p. 014005. DOI: 10.1103/PhysRevFluids.2.014005 (cited on pages 26, 95, 96, 132).

Linne, M. et al. "Correlation of internal flow and spray breakup for a fuel injector used in ship engines". In: 8th US National Combustion Meeting 20132 (2013), pp. 1-8 (cited on page 10).

Linne, Mark. "Analysis of X-ray phase contrast imaging in atomizing sprays". In: Experiments in Fluids 52.5 (2012), pp. 1201-1218. DOI: 10.1007 / s00348-011-1251-7 (cited on page 15).

Linne, Mark. "Imaging in the optically dense regions of a spray: A review of developing techniques". In: Progress in Energy and Combustion Science 39.5 (2013), pp. 403-440. DOI: 10.1016/j.pecs.2013.06.001 (cited on pages $2,6,8,10,12,15,17)$. 
Linne, Mark, Sedarsky, David, Meyer, Terrence, Gord, James, and Carter, Campbell. "Ballistic imaging in the near-field of an effervescent spray". In: Experiments in Fluids 49.4 (2010), pp. 911-923. DOI: 10.1007/s00348010-0883-3 (cited on page 10).

Liu, Xu-Dong, Osher, Stanley, and Chan, Tony. "Weighted Essentially Nonoscillatory Schemes". In: Journal of Computational Physics 115.1 (1994), pp. 200-212. DOI: 10.1006/jcph.1994.1187 (cited on page 50).

Lozano, Antonio, García-Olivares, Antonio, and Dopazo, César. "The instability growth leading to a liquid sheet breakup". In: Physics of Fluids 10.9 (1998), pp. 2188-2197. DOI: 10.1063/1.869740 (cited on pages 24, 26).

$\mathrm{Lu}$, Jiacai and Tryggvason, Gretar. "Direct numerical simulations of multifluid flows in a vertical channel undergoing topology changes". In: Physical Review Fluids 3.8 (2018), pp. 1-20. DOI: 10.1103/PhysRevFluids.3.084401 (cited on page 54).

Lund, T. S., Wu, X., and Squires, K. D. "On the Generation of Turbulent Inflow Conditions for Boundary Layer Simulations". In: Journal of Computational Physics 140 (1998), pp. 233-258. DOI: 10.1006/jcph.1998.5882 (cited on page 70).

Marmottant, P. H. and Villermaux, E. "On spray formation". In: Journal of Fluid Mechanics 498.498 (2004), pp. 73-111. DOI: 10 . 1017 / S0022112003006529 (cited on pages 7, 21).

Marmottant, Philippe. "Atomisation d'un jet liquide par un courant gazeux". PhD thesis. 2001 (cited on pages 7, 173).

Marmottant, Philippe and Villermaux, Emmanuel. "Fragmentaion of stretched liquid ligaments". In: Physics of Fluids 16.8 (2004), pp. 27322741. DOI: $10.1063 / 1.1756030$ (cited on page 7).

Mazzitelli, Irene M. and Lohse, Detlef. "Evolution of energy in flow driven by rising bubbles". In: Physical Review E - Statistical, Nonlinear, and Soft Matter Physics 79.6 (2009), pp. 1-9. DOI: 10.1103/PhysRevE.79.066317 (cited on page 91 ).

Mazzitelli, Irene M., Lohse, Detlef, and Toschi, Federico. "On the relevance of the lift force in bubbly turbulence". In: Journal of Fluid Mechanics 488.488 (2003), pp. 283-313. DOI: 10.1017/S0022112003004877 (cited on pages 91,92$)$. 
Ménard, T, Tanguy, S, and Berlemont, A. "Coupling level set/VOF/ghost fluid methods: Validation and application to 3D simulation of the primary break-up of a liquid jet". In: International Journal of Multiphase Flow 33.5 (2007), pp. 510-524. DOI: 10.1016/j.ijmultiphaseflow . 2006.11.001 (cited on pages 22, 30, 45, 121).

Mendez-Diaz, S., Serrano-García, J. C., Zenit, R., and Hernández-Cordero, J. A. "Power spectral distributions of pseudo-turbulent bubbly flows". In: Physics of Fluids 25.4 (2013). DOI: 10.1063/1. 4800782 (cited on page 91).

Mercado, Julián Martínez, Prakash, Vivek N., Tagawa, Yoshiyuki, Sun, Chao, and Lohse, Detlef. "Lagrangian statistics of light particles in turbulence". In: Physics of Fluids 24.5 (2012). DOI: 10.1063/1.4719148 (cited on page 91).

Milgram, Jerome H. "Short wave damping in the simultaneous presence of a surface film and turbulence". In: Journal of Geophysical Research: Oceans 103.C8 (1998), pp. 15717-15727. DOI: 10 . 1029 / 98jc01191 (cited on page 78).

Moon, S. et al. "Ultrafast X-ray Phase-Contrast Imaging of High-Speed Fuel Sprays from a Two-Hole Diesel Nozzle". In: 22nd Annual Conference on Liquid Atomization and Spray Systems (ILASS Americas 2010) May (2010) (cited on page 16).

Nicoud, F. and Ducros, F. "Subgrid-scale stress modelling based on the square of the velocity gradient tensor". In: Flow, Turbulence and Combustion 62.3 (1999), pp. 183-200. DOI: 10.1023/A: 1009995426001 (cited on page 74).

Noh, W.F. and Woodward, P. SLIC (simple line interface calculation). [Usable in 1, 2, or 3 space dimensions]. Tech. rep. Livermore, CA: Lawrence Livermore National Laboratory (LLNL), 1976. DOI: 10.2172/7261651 (cited on page 57$)$.

"On simulating primary atomization using the refined level set grid method". In: Atomization and Sprays 21.4 (2011), pp. 283-301. DOI: 10.1615 / AtomizSpr.2011002760 (cited on pages 26, 27, 44, 96).

Park, George Ilhwan, Bassenne, Maxime, Urzay, Javier, and Moin, Parviz. "A simple dynamic subgrid-scale model for les of particle-laden turbulence". In: Physical Review Fluids 2.4 (2017), pp. 1-20. DOI: 10.1103 / PhysRevFluids.2.044301 (cited on pages 149, 170, 185).

Payri, R., Tormos, B., Gimeno, J., and Bracho, G. "The potential of Large Eddy Simulation (LES) code for the modeling of flow in diesel injectors". In: Mathematical and Computer Modelling 52.7-8 (2010), pp. 1151-1160. DOI: $10.1016 / \mathrm{j} . \mathrm{mcm} .2010 .02 .033$ (cited on page 30). 
Payri, Raul, Bracho, Gabriela, Marti-Aldaravi, Pedro, and Viera, Alberto. "Near Field Visualization of Diesel Spray for Different Nozzle Inclination Angles in Non-Vaporizing Conditions". In: Atomization and Sprays 27.3 (2017), pp. 251-267. DOI: 10 . 1615 / atomizspr . 2017017949 (cited on page 127).

Payri, Raul, Gimeno, Jaime, Bracho, Gabriela, and Bautista, Abian. "Virtual injection modelling for ECN Spray G". In: Iclass 2018. Chicago, 2018, pp. 1-8 (cited on page 7).

Payri, Raul, Gimeno, Jaime, Marti-Aldaravi, Pedro, and Bracho, Gabriela. "Study of the influence of the inlet boundary conditions in a LES simulation of internal flow in a diesel injector". In: Mathematical and Computer Modelling 57.7-8 (2013), pp. 1709-1715. DOI: 10.1016/j.mcm.2011.11. 019 (cited on page 30).

Payri, Raul, Salvador, Javier, Gimeno, J., and De la Morena, J. "Analysis of Diesel Spray Atomization By Means of a Near-Nozzle Field Visualization Technique". In: Atomization and Sprays 21.9 (2012), pp. 753-774. DOI: 10.1615/atomizspr. 2012004051 (cited on pages 113, 128).

Perret, Laurent, Delville, Joël, Manceau, Rémi, and Bonnet, Jean Paul. “Turbulent inflow conditions for large-eddy simulation based on low-order empirical model". In: Physics of Fluids 20.7 (2008). DOI: 10.1063/1.2957019 (cited on page 68).

Pickett, Lyle M., Manin, Julien, Kastengren, Alan L, and Powell, Christopher F. "Comparison of Near-Field Structure and Growth of a Diesel Spray Using Light-Based Optical Microscopy and X-Ray Radiography". In: SAE Technical Paper 2014-01-1412 7.2 (2014), pp. 1044-1053. DOI: 10.4271/ 2014-01-1412 (cited on pages 13, 17, 18).

Pickett, Lyle M. et al. "Comparison of Diesel Spray Combustion in Different High-Temperature, High-Pressure Facilities". In: SAE International Journal of Engines 3.2 (2010), pp. 156-181. DOI: $10.4271 / 2010-01-2106$ (cited on page 114).

"Piecewise Linear Interface Calculation". In: Comptes Rendus - Academie des Sciences, Serie II: Mecanique, Physique, Chimie, Astronomie 320.8 (1995), pp. 391-396 (cited on page 57).

Pitsch, H. and Desjardins, Olivier. "Detailed Numerical Investigation of Turbulent Atomization of Liquid Jets". In: Atomization and Sprays 20.4 (2010), pp. 311-336. DOI: 10 . 1615/atomizspr . v20 . i4 . 40 (cited on pages $7,26,28,31,32,44,94,96,148,185)$. 
Pope, Stephen B. Turbulent Flows. 2001. DOI: 10.1088/0957-0233/12/11/ 705 (cited on pages 2, 5, 6, 21, 26, 69, 73, 79, 88, 90, 98, 114, 134, 153, 166).

Popinet, Stéphane. "An accurate adaptive solver for surface-tension-driven interfacial flows". In: Journal of Computational Physics 228.16 (2009), pp. 5838-5866. DOI: $10.1016 / \mathrm{j} \cdot \mathrm{jcp} .2009 .04 .042$ (cited on page 61).

Popinet, Stéphane. Gerris code. 2010 (cited on pages 44, 50).

Popinet, Stéphane. Basilisk code. 2014 (cited on pages 44, 50).

Popinet, Stéphane. "Numerical Models of Surface Tension". In: Annual Review of Fluid Mechanics 50.1 (2017), pp. 49-75. DOI: 10.1146/annurev-fluid122316-045034 (cited on pages 46, 58, 59).

Prakash, Vivek N. et al. "Energy spectra in turbulent bubbly flows". In: Journal of Fluid Mechanics 791 (2016), pp. 174-190. DOI: 10.1017/jfm. 2016. 49 (cited on pages 22, 91, 169).

Rayleigh, Lord. "On the instability of jets". In: Proceedings of the London Mathematical Society s1-10.1 (1878), pp. 4-13. DOI: 10.1112/plms/s110.1.4 (cited on page 2).

Reitz, R. D. and Bracco, F. V. "Mechanism of atomization of a liquid jet". In: Physics of Fluids 25.10 (1982), pp. 1730-1742. DOI: 10.1063/1.863650 (cited on page 2).

Richardson, Lewis F. "Weather prediction by numerical process, second edition". In: Weather Prediction by Numerical Process, Second Edition (1922), pp. 1-236. DOI: 10.1017/CB09780511618291 (cited on pages 86, 88).

Roghair, Ivo et al. "Energy spectra and bubble velocity distributions in pseudo-turbulence: Numerical simulations vs. experiments". In: International Journal of Multiphase Flow 37.9 (2011), pp. 1093-1098. DOI: 10 . 1016/j.ijmultiphaseflow.2011.07.004 (cited on page 91).

Sadeghi, H. and Pollard, Andrew. "Axial Velocity Spectra Scaling in a Round, Free Jet". In: Proceeding of THMT-12. Proceedings of the Seventh International Symposium On Turbulence, Heat and Mass Transfer Palermo, Italy, 24-27 September, 2012 November (2014), p. 11. DOI: 10.1615/ichmt. 2012. procsevintsympturbheattransf pal. 1220 (cited on pages 98, 99).

Sahu, S., Hardalupas, Y., and Taylor, A. M.K.P. "Droplet-turbulence interaction in a confined polydispersed spray: Effect of turbulence on droplet dispersion". In: Journal of Fluid Mechanics 794 (2016), pp. 267-309. DOI: 10.1017/jfm.2016.169 (cited on page 185). 
Salvador, F.J., Ruiz, S., Crialesi-Esposito, Marco, and Blanquer, Ignacio. "Analysis on the Effects of Turbulent Inflow Conditions on Spray Primary Atomization in the Near-Field by Direct Numerical Simulation". In: International Journal of Multiphase Flow 102 (2018), pp. 49-63. DOI: 10.1016/j.ijmultiphaseflow. 2018.01.019 (cited on pages 106, 169, 174).

Scardovelli, Ruben and Zaleski, Stephane. "Interface reconstruction with leastsquare fit and split Eulerian-Lagrangian advection". In: International Journal for Numerical Methods in Fluids 41.3 (2003), pp. 251-274. DOI: 10.1002/fld. 431 (cited on page 57).

Scardovelli, Ruben and Zaleski, Stéphane. "Direct Numerical Simulation of Free-Surface and Interfacial Flow". In: Annual Review of Fluid Mechanics 31.1 (1999), pp. 567-603. DOI: 10.1146/annurev.fluid.31.1.567 (cited on pages 46,57$)$.

Shinjo, J. and Umemura, A. "Detailed simulation of primary atomization mechanisms in Diesel jet sprays (isolated identification of liquid jet tip effects)". In: Proceedings of the Combustion Institute 33.2 (2011), pp. 20892097. DOI: $10.1016 / \mathrm{j}$.proci.2010.07.006 (cited on pages $21,23,25,30$, $95,106,132,174,177)$.

Shinjo, J. and Umemura, A. "Surface instability and primary atomization characteristics of straight liquid jet sprays". In: International Journal of Multiphase Flow 37.10 (2011), pp. 1294-1304. DOI: 10 . 1016 / j . ijmultiphaseflow.2011.08.002 (cited on pages 23, 24).

Shinjo, J. and Umemura, Akira. "Simulation of liquid jet primary breakup: Dynamics of ligament and droplet formation". In: International Journal of Multiphase Flow 36.7 (2010), pp. 513-532. DOI: $10.1016 / \mathrm{j}$. ijmultiphaseflow.2010.03.008 (cited on pages 22, 71, 95, 104, 120, $126,127,130)$.

Shinjo, J., Xia, J., and Umemura, A. "Droplet/ligament modulation of local small-scale turbulence and scalar mixing in a dense fuel spray". In: Proceedings of the Combustion Institute 35.2 (2015), pp. 1595-1602. DOI: 10.1016/j.proci.2014.06.088 (cited on pages 120, 126, 127).

Shu, Chi Wang and Osher, Stanley. "Efficient implementation of essentially non-oscillatory shock-capturing schemes". In: Journal of Computational Physics 77.2 (1988), pp. 439-471. DOI: 10.1016/0021-9991(88)90177-5 (cited on page 50). 
Sirignano, W. A. and Mehring, C. "Review of theory of distortion and disintegration of liquid streams". In: Progress in Energy and Combustion Science 26.4 (2000), pp. 609-655. DOI: 10.1016/S0360-1285(00) 00014-9 (cited on page 1).

STANLEY, S. A., SARKAR, S., and MELLADO, J. P. "A study of the flowfield evolution and mixing in a planar turbulent jet using direct numerical simulation". In: Journal of Fluid Mechanics 450.January 2002 (2002), pp. 377-407. DOI: 10.1017/s0022112001006644 (cited on page 101).

Subramaniam, S. "Statistical representation of a spray as a point process". In: Physics of Fluids 12.10 (2000), pp. 2413-2431. DOI: 10.1063/1.1288266 (cited on pages $96,170,183$ ).

Subramaniam, S. "Statistical modeling of sprays using the droplet distribution function". In: Physics of Fluids 13.3 (2001), pp. 624-642. DOI: 10.1063/ 1.1344893 (cited on page 96).

Sundaram, Shivshankar and Collins, Lance R. "A numerical study of the modulation of isotropic turbulence by suspended particles". In: Journal of Fluid Mechanics 379 (1999), pp. 105-143. DOI: 10.1017/S0022112098003073 (cited on pages 92,169 ).

Sussman, Mark. "A level set approach for computing solutions to incompressible two-phase flow". In: Journal of Computational Physics 114.1 (1994), pp. 146-159. DOI: 10.1006/jcph.1994.1155 (cited on pages 57, 58).

Tabor, G. R. and Baba-Ahmadi, M. H. "Inlet conditions for large eddy simulation: A review". In: Computers and Fluids 39.4 (2010), pp. 553-567. DOI: $10.1016 /$ j.compfluid.2009.10.007 (cited on page 68).

"The fractal geometry of round turbulent cryogenic nitrogen jets at subcritical and supercritical pressures". In: Atomization and Sprays 14.1 (2004), pp. 81-91. DOI: 10.1615/AtomizSpr.v14.i1.50 (cited on page 173).

Tong, Chenning and Warhaft, Z. "Passive Scalar Dispersion and Mixing in a Turbulent Jet". In: Journal of Fluid Mechanics 292.-1 (1995), pp. 1-38. DOI: $10.1017 / \mathrm{S} 0022112095001418$ (cited on page 99).

Toschi, Federico and Bodenschatz, Eberhard. "Lagrangian Properties of Particles in Turbulence". In: Annual Review of Fluid Mechanics 41.1 (2009), pp. 375-404. DOI: 10.1146/annurev . fluid.010908.165210 (cited on page 92$)$. 
Trask, Nathaniel, Schmidt, David P., Lightfoot, Malissa, and Danczyk, Stephen. "Compressible Modeling of the Internal Two-Phase Flow in a Gas-Centered Swirl Coaxial Fuel Injector". In: Journal of Propulsion and Power 28.4 (2012), pp. 685-693. DOI: 10 . 2514/1 . b34102 (cited on page 102).

Tryggvason, G. et al. "A Front-Tracking Method for the Computations of Multiphase Flow". In: Journal of Computational Physics 169.2 (2001), pp. 708-759. DOI: 10.1006/jcph.2001.6726 (cited on page 54).

Tryggvason, Grétar, Scardovelli, Ruben, and Zaleski, Stéphane. Direct numerical simulations of gas-liquid multiphase flows. Cambridge University Press, 2011 (cited on pages 46, 47, 49, 51, 52, 56-59).

Uberoi, Mahinder S. and Freymuth, Peter. "Turbulent energy balance and spectra of the axisymmetric wake". In: Physics of Fluids 13.9 (1970), pp. 2205-2210. DOI: 10.1063/1.1693225 (cited on pages 153, 154).

Umemura, Akira. "Turbulent atomization subgrid model for two-phase flow large eddy simulation (theoretical development)". In: Combustion and Flame 165 (2016), pp. 154-176. DOI: 10.1016/j . combustflame. 2015. 11.025 (cited on page 23).

Umemura, Akira and Shinjo, Junji. "Detailed SGS atomization model and its implementation to two-phase flow LES". In: Combustion and Flame 195 (2018), pp. 232-252. DOI: 10.1016/j . combustflame.2018.01.026 (cited on page 23$)$.

Unverdi, S O and Tryggvason, G. "A front-tracking method for viscous, incompressible, multi-fluid flows". In: J. Comput. Phys. 100 (1992), pp. 2537 (cited on page 54).

Vassallo, P. and Ashgriz, N. "Satellite Formation and Merging in Liquid Jet Breakup". In: Proceedings of the Royal Society A: Mathematical, Physical and Engineering Sciences 433.1888 (2006), pp. 269-286. DOI: $10.1098 /$ rspa.1991.0047 (cited on pages 31, 86, 129, 130).

Vaudor, G., Ménard, T., Aniszewski, W., Doring, M., and Berlemont, A. "A consistent mass and momentum flux computation method for two phase flows. Application to atomization process". In: Computers and Fluids 152 (2017), pp. 204-216. DOI: 10.1016/j.compfluid.2017.04.023 (cited on pages 20, 25).

Veeravallif, Srtntvas V. "Local Isotropy in Turbulent Boundary Layers at High Reynolds Number". In: Journal of Fluid Mechanics 268.-1 (1994), pp. 333372. DOI: $10.1017 /$ S0022112094001370 (cited on pages 153,154 ). 
Vorst, H. A. van der. "Bi-CGSTAB: A Fast and Smoothly Converging Variant of Bi-CG for the Solution of Nonsymmetric Linear Systems". In: SIAM Journal on Scientific and Statistical Computing 13.2 (2005), pp. 631-644. DOI: $10.1137 / 0913035$ (cited on page 52).

Wang, Yujie et al. "Ultrafast X-ray study of dense-liquid-jet flow dynamics using structure-tracking velocimetry". In: Nature Physics 4.4 (2008), pp. 305-309. DOI: 10.1038/nphys840 (cited on pages 14-16).

Wilson, S. D. R. and Hulme, A. "The Effect of Bubbles Attached to an Electrode on Electrical Resistance and Dissolved Gas Concentration". In: Proceedings of the Royal Society A: Mathematical, Physical and Engineering Sciences 387.1792 (1983), pp. 133-146. DOI: 10.1098/rspa. 1983.0054 (cited on pages 87, 99, 103).

Wilson, S. D. R. and Hulme, A. "The Effect of Bubbles Attached to an Electrode on Electrical Resistance and Dissolved Gas Concentration". In: Proceedings of the Royal Society A: Mathematical, Physical and Engineering Sciences 387.1792 (1983), pp. 133-146. DOI: 10.1098/rspa.1983.0054 (cited on pages 91,98 ).

Wu, Xiaohua, Baltzer, J. R., and Adrian, R. J. "Direct numerical simulation of a 30R long turbulent pipe flow at $\mathrm{R}+=685$ : Large-and very large-scale motions". In: Journal of Fluid Mechanics 698 (2012), pp. 235-281. DOI: 10.1017/jfm.2012.81 (cited on page 74).

$\mathrm{Wu}$, Xiaohua and Moin, Parviz. A direct numerical simulation study on the mean velocity characteristics in turbulent pipe flow. Vol. 608. 2008, pp. 81112. DOI: 10.1017/S0022112008002085 (cited on pages 74, 75).

Youngs, D. "Them dependent multimaterial flow with large fluid distribution". In: Numer. Methods Fluid D 24.273-285 (1982) (cited on page 57).

Zaleski, S, Tryggvason, G, and Scardovelli, R. ParisSimulator code. 2013 (cited on page 45).

Zandian, A., Sirignano, W. A., and Hussain, F. "Planar liquid jet: Early deformation and atomization cascades". In: Physics of Fluids 29.6 (2017). DOI: $10.1063 / 1.4986790$ (cited on page 25).

Zandian, A., Sirignano, W. A., and Hussain, F. "Understanding liquid-jet atomization cascades via vortex dynamics". In: Journal of Fluid Mechanics 843 (2018), pp. 293-354. DOI: 10.1017/jfm.2018.113 (cited on pages 24, $25,31,169)$. 
Ziaee, Ahmad et al. "Spray drying of pharmaceuticals and biopharmaceuticals: Critical parameters and experimental process optimization approaches". In: European Journal of Pharmaceutical Sciences 127.November (2019), pp. 300-318. DOI: 10.1016/j.ejps.2018.10.026 (cited on page 1). 